MÉMOIRES DE LA SMF 98

\title{
COBORDISME COMPLEXE DES ESPACES PROFINIS ET FONCTEUR T DE LANNES
}

François-Xavier Dehon

Société Mathématique de France 2004

Publié avec le concours du Centre National de la Recherche Scientifique 
F.-X. Dehon

UMR $6621 \mathrm{du}$ CNRS, Laboratoire J.A. Dieudonné,

Université de Nice-Sophia-Antipolis, Parc Valrose, 06108 Nice cedex 2, France.

E-mail : dehon@math.unice.fr

Classification mathématique par sujets (2000). - 55Q05; 18C15, 55N22, 55R37, 55S25, $55 \mathrm{Uxx}$.

Mots clefs. - Algèbres sur une monade, algèbres instables, classes d'homotopie d'applications, cobordisme complexe, espaces classifiants, espaces fonctionnels, espaces profinis, foncteur $\mathrm{T}$, résolutions.

L'auteur a bénéficié pendant la réalisation de ce travail d'une bourse individuelle Marie Curie de la Commission européenne (HPMF-CT-1999-00135). 


\title{
COBORDISME COMPLEXE DES ESPACES PROFINIS ET FONCTEUR T DE LANNES
}

\author{
François-Xavier Dehon
}

Résumé. - Nous montrons dans ce mémoire que la MU-cohomologie continue des espaces fonctionnels de source le classifiant $\mathrm{B} \pi$ d'un groupe de Lie compact commutatif et de but le pro- $p$-complété d'un espace dont la cohomologie à coefficients dans les entiers $p$-adiques est sans torsion est l'image de la MU-cohomologie complétée en $p$ de l'espace $\mathrm{au}$ but par un foncteur $\mathrm{T}_{\mathrm{B} \pi}$ analogue au foncteur $\mathrm{T}$ associé à la cohomologie modulo $p$ du classifiant du groupe cyclique d'ordre $p$.

\section{Abstract (Complex cobordism of profinite spaces and Lannes' T-functor)}

We show in this paper that the continuous MU-cohomology of the mapping spaces from the classifying space $\mathrm{B} \pi$ of some commutative compact Lie group to the pro$p$-completion of a space whose $p$-adic cohomology is torsion free is the image of the $p$-completed MU-cohomology of the target space by a functor $\mathrm{T}_{\mathrm{B} \pi}$ analogous to the functor T associated to the classifying space of the cyclic group of order $p$. 



\section{TABLE DES MATIÈRES}

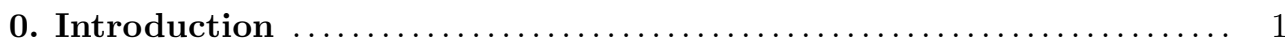

1. Cobordisme complexe des espaces profinis $\ldots \ldots \ldots \ldots \ldots \ldots \ldots \ldots$

1. Théorie homotopique des espaces profinis $\ldots \ldots \ldots \ldots \ldots \ldots \ldots \ldots \ldots$

1.1. Rappel sur la $p$-complétion profinie (d'après $[\mathbf{M o 2}]$ ) $\ldots \ldots \ldots \ldots \ldots \ldots$

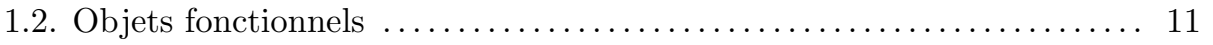

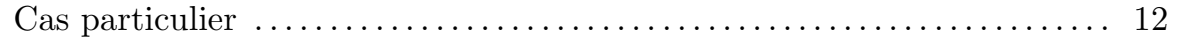

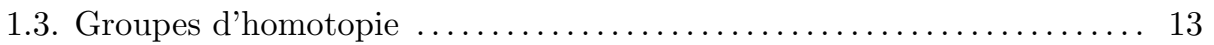

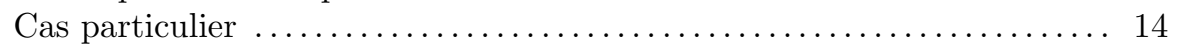

1.4. Quelques limites et colimites homotopiques $\ldots \ldots \ldots \ldots \ldots \ldots \ldots \ldots . \ldots 17$

1.5. Action d'un pro- $p$-groupe abélien simplicial $\ldots \ldots \ldots \ldots \ldots \ldots \ldots \ldots \ldots 20$

1.6. Cohomologie continue des espaces profinis $\ldots \ldots \ldots \ldots \ldots \ldots \ldots \ldots .22$

2. Structure additive de la MU-cohomologie continue des espaces profinis . . 25

2.1. $\mathrm{MU}^{*}$-modules filtrés libres et espaces profinis sans $p$-torsion $\ldots \ldots \ldots \ldots 27$

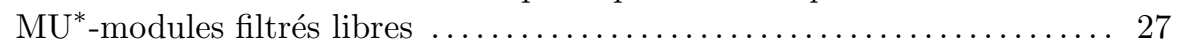

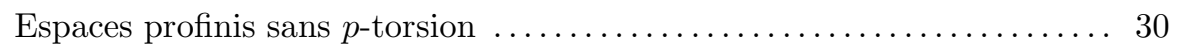

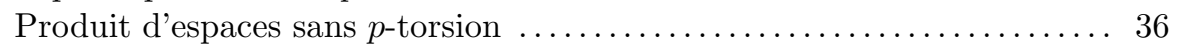

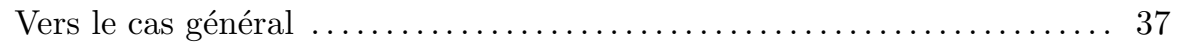

2.2. $\mathrm{MU}^{*}$-modules filtrés à présentation libre et MU-cohomologie continue des espaces profinis dans le cas général .................. 38

1 -complexes de $\widehat{\mathcal{L}}$ et $\widehat{\mathrm{L}}$-algèbres $\ldots \ldots \ldots \ldots \ldots \ldots \ldots \ldots \ldots \ldots \ldots \ldots$

$\widehat{\mathrm{L}}$-algèbres, structure de $\mathrm{MU}^{*}$-module et filtration $\ldots \ldots \ldots \ldots \ldots \ldots \ldots . \ldots 39$

Filtration squelettale d'une $\widehat{\mathrm{L}}$-algèbre $\ldots \ldots \ldots \ldots \ldots \ldots \ldots \ldots \ldots \ldots \ldots \ldots .42$

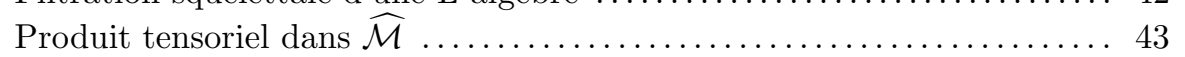

Présentation de la MU-cohomologie continue des espaces profinis . . . . 44

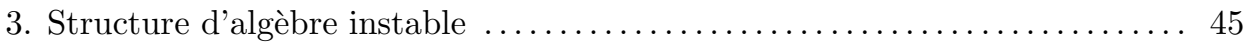

3.1. Espaces d'Eilenberg-Mac Lane généralisés et MU-algèbres instables . 46

3.2. Comparaison avec les algèbres instables pour la cohomologie modulo $p \quad 51$

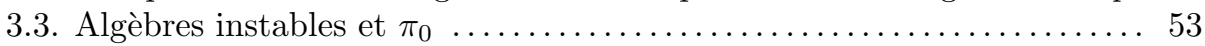




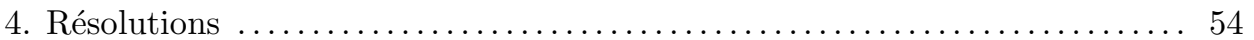

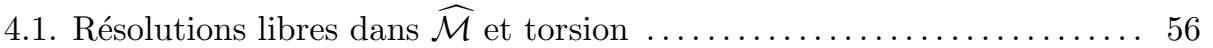

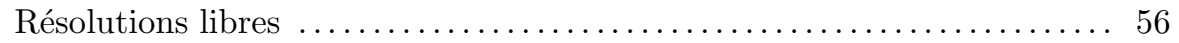

Exactitude du produit tensoriel et torsion $\ldots \ldots \ldots \ldots \ldots \ldots \ldots \ldots \ldots \ldots . \ldots 9$

Comparaison avec les modules $\operatorname{Tor}_{*}^{\widehat{\mathrm{MU}}^{*}}(M, N) \ldots \ldots \ldots \ldots \ldots \ldots \ldots \ldots$

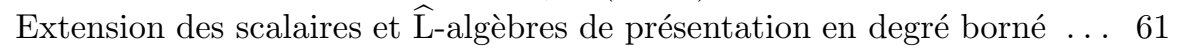

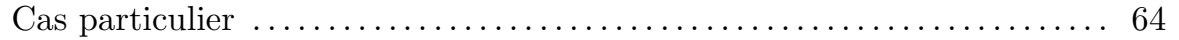

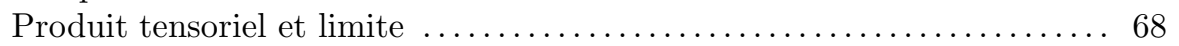

4.2. Résolutions instables et résolutions des espaces $\ldots \ldots \ldots \ldots \ldots \ldots \ldots 72$

4.3. MU-résolutions et suites spectrales $\ldots \ldots \ldots \ldots \ldots \ldots \ldots \ldots \ldots \ldots \ldots$

5. Cohomologie des espaces fonctionnels et foncteurs de division . . . . . . . 79

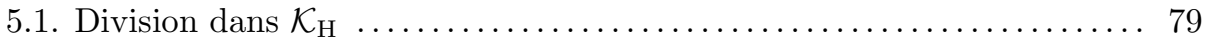

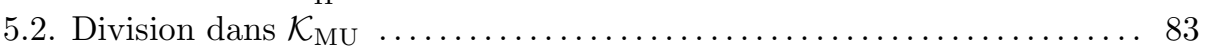

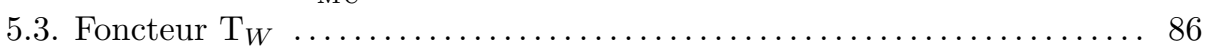

2. Cohomologie des espaces fonctionnels de source le classifiant d'un groupe de Lie compact commutatif $\ldots \ldots \ldots \ldots \ldots \ldots \ldots \ldots \ldots \ldots \ldots$

6. Propriétés de la cohomologie modulo $p$ des espaces fonctionnels de source le

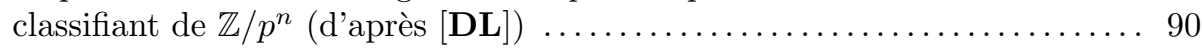

7. MU-cohomologie des espaces fonctionnels de source le classifiant d'un groupe

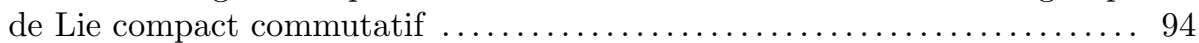

7.1. Foncteurs $\mathrm{T}_{n}$ et cohomologie des espaces fonctionnels . . . . . . . . . . 94

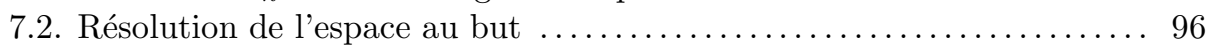

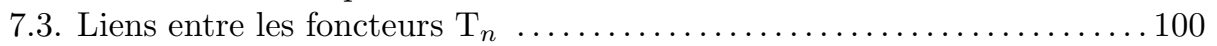

8. MU-cohomologie du classifiant de $\mathbb{Z} / p^{n}$ et foncteur $\mathrm{T}_{n} \ldots \ldots \ldots \ldots \ldots \ldots . \ldots 3$

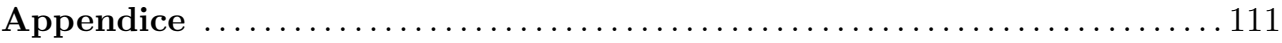

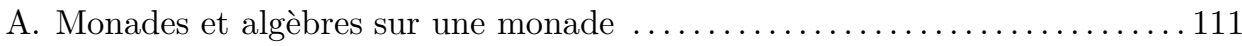

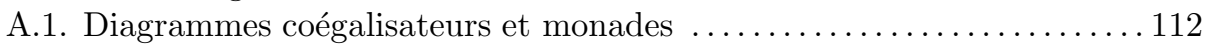

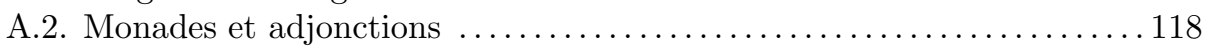

A.3. Application : Monades et catégories abéliennes. .................. 122

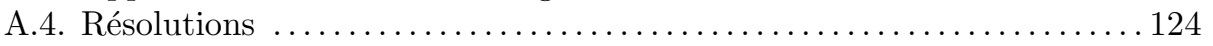

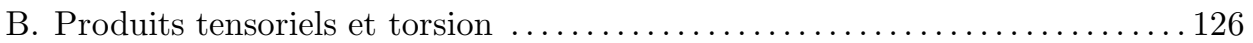

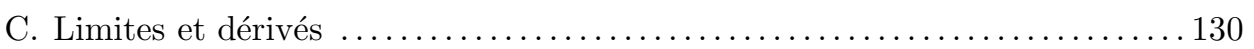

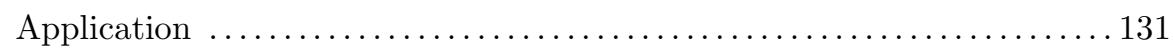

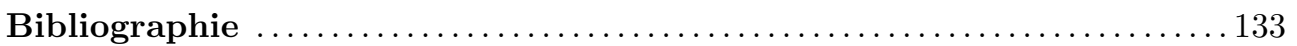

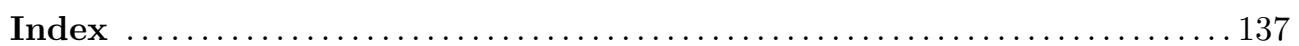




\section{CHAPITRE 0}

\section{INTRODUCTION}

Ce mémoire fait suite à $[\mathbf{D L}]$ (et $[\mathbf{K W}]$ ). Soient $p$ un nombre premier fixé, $\pi$ un groupe de Lie compact commutatif, $X$ un espace dont la cohomologie à coefficients dans l'anneau des entiers $p$-adiques est sans torsion et désignons par MU le spectre représentant le cobordisme complexe. Nous montrons que la MU-cohomologie complétée en $p$ continue de l'espace fonctionnel de source le classifiant $\mathrm{B} \pi$ de $\pi$ et de but le $p$-complété profini de $X$ est l'image par un foncteur $\mathrm{T}_{\mathrm{B} \pi}$ de la MU-cohomologie complétée en $p$ de $X$, et étendons ainsi les résultats de J. Lannes ([La1]) concernant la cohomologie modulo $p$ des espaces fonctionnels de source le classifiant d'un produit de groupes cycliques d'ordre $p$.

Indiquons ce dont il s'agit :

Notre théorie homotopique est celle des ensembles profinis simpliciaux (ou espaces profinis) où les équivalences faibles sont les applications simpliciales continues induisant un isomorphisme en cohomologie modulo $p$ continue ([Mo2]). On note h $\widehat{\mathcal{S}}$ la catégorie homotopique associée. (La raison pour laquelle nous avons besoin des espaces profinis et de leurs cohomologies continues plutôt que des espaces et cohomologies ordinaires apparaîtra ci-dessous.)

Soient $W$ un ensemble simplicial qu'on écrit comme la colimite de ses sous ensembles finis simpliciaux $W_{\alpha}$, et $X$ un espace profini. L'espace profini fonctionnel $\operatorname{hom}(W, X)$ est l'adjoint à droite en $X$ du foncteur $\widehat{S} \rightarrow \widehat{S}, Z \mapsto W \widehat{\times} Z=$ $\operatorname{colim}_{\alpha}\left(W_{\alpha} \times Z\right)$. Si $X$ est fibrant, l'ensemble (profini) $\pi_{0} \operatorname{hom}(W, X)$ s'identifie à l'ensemble $[W,|X|]$ des classes d'homotopie simpliciale d'applications de $W$ dans l'espace sous-jacent à $X$, donc à l'ensemble des morphismes $\operatorname{Hom}_{\mathrm{h} \widehat{\mathcal{S}}}(W \widehat{\times} \mathrm{pt}, X)$, où $W \widehat{\times} \mathrm{pt}$ est le complété profini de $W$. (Voir les sections 1.2 et 1.3.)

Notons $\mathrm{H}^{*} Z$ la cohomologie modulo $p$ continue d'un espace profini $Z, \mathcal{E}$ la catégorie des $\mathbb{F}_{p}$-espaces vectoriels gradués et soit $E$ un objet de $\mathcal{E}$. Il existe un espace profini $\mathrm{K}_{\mathrm{H}}(E)$ et un morphisme $E \rightarrow \mathrm{H}^{*} \mathrm{~K}_{\mathrm{H}}(E)$ induisant une bijection $\operatorname{Hom}_{\mathrm{h} \widehat{\mathcal{S}}}\left(Z, \mathrm{~K}_{\mathrm{H}}(E)\right) \cong$ $\operatorname{Hom}_{\mathcal{E}}\left(E, \mathrm{H}^{*} Z\right)$ naturelle en $Z \in \mathrm{h} \widehat{\mathcal{S}}$. La catégorie $\mathcal{K}_{\mathrm{H}}$ des algèbres instables sur 
l'algèbre de Steenrod modulo $p$ coïncide avec la catégorie des algèbres associées à la monade $E \mapsto \mathrm{H}^{*} \mathrm{~K}_{\mathrm{H}}(E)$ sur $\mathcal{E}$, de sorte que l'application

$$
\operatorname{Hom}_{\mathrm{h} \widehat{\mathcal{S}}}(Z, Y) \longrightarrow \operatorname{Hom}_{\mathcal{K}_{\mathrm{H}}}\left(\mathrm{H}^{*} Y, \mathrm{H}^{*} Z\right)
$$

est une bijection pour tout espace profini $Z$ lorsque $Y$ est isomorphe à $\mathrm{K}_{\mathrm{H}}(E)$ pour un $E \in \mathcal{E}$ ([La2], voir la section 3.2).

Si la cohomologie modulo $p$ de l'espace $W$ est finie en chaque degré alors on a pour tout espace profini $Z$ un isomorphisme de Künneth $\mathrm{H}^{*} W \otimes \mathrm{H}^{*} Z \stackrel{\sim}{\longrightarrow} \mathrm{H}^{*} W \widehat{\times} Z$ (lemme 5.1.3) et l'endofoncteur $N \mapsto \mathrm{H}^{*} W \otimes N$ de $\mathcal{K}_{\mathrm{H}}$ admet un adjoint à gauche $\left(-: \mathrm{H}^{*} W\right)_{\mathcal{K}_{\mathrm{H}}}$. L'application d'évaluation $W \widehat{\times} \operatorname{hom}(W, X) \rightarrow X$ induit un morphisme

$$
\left(\mathrm{H}^{*} X: \mathrm{H}^{*} W\right)_{\mathcal{K}_{\mathrm{H}}} \longrightarrow \mathrm{H}^{*} \operatorname{hom}(W, X)
$$

qui est un isomorphisme si $X$ est fibrant dans $\widehat{S}$ et isomorphe dans h $\widehat{\mathcal{S}}$ à $\mathrm{K}_{\mathrm{H}}(E)$ pour un $E \in \mathcal{E}$. (Il faudrait imposer $\mathrm{H}^{*} W$ de dimension totale finie ou $E$ nul en degré assez grand pour avoir un tel isomorphisme si on utilisait les espaces et la cohomologie modulo $p$ ordinaires.) (Voir la section 5.1.)

Lorsque $W$ est le classifiant $\mathrm{B} V$ d'un produit fini de groupes cycliques d'ordre $p$, l'application

$$
[\mathrm{B} V,|X|] \longrightarrow \operatorname{Hom}_{\mathcal{K}_{\mathrm{H}}}\left(\mathrm{H}^{*} X, \mathrm{H}^{*} \mathrm{~B} V\right)
$$

est une bijection et le morphisme

$$
\left(\mathrm{H}^{*} X: \mathrm{H}^{*} \mathrm{~B} V\right)_{\mathcal{K}_{\mathrm{H}}} \longrightarrow \mathrm{H}^{*} \operatorname{hom}(\mathrm{B} V, X)
$$

est un isomorphisme pour tout espace profini fibrant $X$ ([La1], [Mo2]). Ceci vient des propriétés exceptionnelles de la cohomologie modulo $p$ de $\mathrm{B} V$ comme module et algèbre instable sur l'algèbre de Steenrod ([La1 $]$ ). (A nouveau il faudrait imposer des hypothèses de finitude sur $X$ si on utilisait les espaces et la cohomologie modulo $p$ ordinaires.)

Dans [DL] on montre pour $\pi$ un groupe de Lie compact commutatif que la cohomologie modulo $p$ continue de l'espace fonctionnel hom $(\mathrm{B} \pi, X)$ possède des propriétés d'exactitude en la cohomologie modulo $p$ de $X$ lorsque la cohomologie à coefficients $p$-adiques de $X$ est sans torsion (on dit alors que $X$ est sans $p$-torsion), puis on reprend l'idée de Kuhn et Winstead ([KW]) d'utiliser le début d'une MU-résolution de l'espace au but $X$ : Les propriétés d'exactitude mises en évidence et le fait que les espaces formant le début de cette MU-résolution sont également sans $p$-torsion font que l'ensemble des classes d'homotopie d'applications $[\mathrm{B} \pi,|X|]$ s'exprime en terme de la structure d'algèbre instable des MU-cohomologies complétées en $p$ de $X$ et de $\mathrm{B} \pi$.

Notons $\widehat{\mathrm{MU}}^{*} Z$ la MU-cohomologie (complétée en $p$ ) continue d'un espace profini $Z$ (voir la section 1.6), Ens-gr la catégorie des ensembles $\mathbb{Z}$-gradués et soit $S$ un objet de $\mathcal{E}$ ns-gr. Il existe un espace profini $\mathrm{K}(S)$ et un morphisme $S \rightarrow \widehat{\mathrm{MU}}^{*} \mathrm{~K}(S)$ induisant une bijection $\operatorname{Hom}_{\mathrm{h} \widehat{\mathcal{S}}}(Z, \mathrm{~K}(S)) \cong \operatorname{Hom}_{\mathcal{E} n \text { s-gr }}\left(S, \widehat{\mathrm{MU}}^{*} Z\right)$ naturelle en $Z \in \mathrm{h} \widehat{\mathcal{S}}$. On définit la 
catégorie $\mathcal{K}_{\mathrm{MU}}$ des MU-algèbres instables comme la catégorie des algèbres associées à la monade $S \mapsto \widehat{\mathrm{MU}}^{*} \mathrm{~K}(S)$ sur Ens-gr, de sorte que l'application

$$
\operatorname{Hom}_{\mathrm{h} \widehat{\mathcal{S}}}(Z, Y) \longrightarrow \operatorname{Hom}_{\mathcal{K}_{\mathrm{MU}}}\left(\widehat{\mathrm{MU}}^{*} Y, \widehat{\mathrm{MU}}^{*} Z\right)
$$

est une bijection pour tout espace profini $Z$ lorsque $Y$ est isomorphe à $\mathrm{K}(S)$ pour un $S \in \mathcal{E}$ ns-gr.

Nous définissons une catégorie abélienne $\widehat{\mathcal{M}}$ munie d'un produit tensoriel $\widehat{\otimes}$ avec les propriétés suivantes :

(1) La MU-cohomologie continue d'un espace profini $Z$ est naturellement un objet de $\widehat{\mathcal{M}}$ et en est un objet projectif si la cohomologie continue à coefficients $p$-adiques de $Z$ est sans torsion. (Voir la section 2.)

(2) La structure de spectre en anneau de MU induit pour tout espace $W$ et pour tout espace profini $Z$ un morphisme $\widehat{\mathrm{MU}}^{*} W \widehat{\otimes} \widehat{\mathrm{MU}}^{*} Z \rightarrow \widehat{\mathrm{MU}}^{*}(W \widehat{\times} Z)$ qui est un isomorphisme si la cohomologie à coefficients $p$-adiques de $W$ est sans torsion et de type fini en chaque degré (lemme 5.2.1).

(3) Soit $W$ un espace dont la cohomologie à coefficients $p$-adiques est sans torsion et de type fini en chaque degré; alors l'endofoncteur $N \mapsto \widehat{\mathrm{MU}}^{*} W \widehat{\otimes} N$ de $\mathcal{K}_{\mathrm{MU}}$ admet un adjoint à gauche $\left(-: \widehat{\mathrm{MU}}^{*} W\right)_{\mathcal{K}_{\mathrm{MU}}}$. L'application d'évaluation $W \widehat{\times} \operatorname{hom}(W, X) \rightarrow X$ induit un morphisme

$$
\left(\widehat{\mathrm{MU}}^{*} X: \widehat{\mathrm{MU}}^{*} W\right)_{\mathcal{K}_{\mathrm{MU}}} \longrightarrow \widehat{\mathrm{MU}}^{*} \operatorname{hom}(W, X)
$$

qui est un isomorphisme si $X$ est fibrant dans $\widehat{\mathcal{S}}$ et isomorphe dans h $\widehat{\mathcal{S}}$ à $\mathrm{K}(S)$ pour un $S \in \mathcal{E}$ ns-gr. (Voir la section 5.2.)

L'un des principaux résultats de ce mémoire est le suivant (voir la section 7) :

ThÉORÈme 0.1. - Soient $T$ un tore, BT son classifiant et $X$ un espace profini fibrant dont la cohomologie continue à coefficients p-adiques est sans torsion; alors le morphisme

$$
\left(\widehat{\mathrm{MU}}^{*} X: \widehat{\mathrm{MU}}^{*} \mathrm{~B} T\right)_{\mathcal{K}_{\mathrm{MU}}} \longrightarrow \widehat{\mathrm{MU}}^{*} \operatorname{hom}(\mathrm{B} T, X)
$$

est un isomorphisme.

Nous n'avons pas besoin d'expliciter la structure de MU-algèbre instable pour obtenir ce résultat. Nous renvoyons le lecteur à $[\mathbf{R W}]$ et $[\mathbf{B J W}]$ pour une telle étude. La définition de la catégorie $\mathcal{K}_{\mathrm{MU}}$ est formelle et ne dépend pas de propriétés du spectre MU autres que l'existence d'une MU-cohomologie continue. Cette existence est garantie par le fait que le $n$-ième terme du $\Omega$-spectre associé à MU est, pour $n$ assez grand, connexe et de cohomologie modulo $p$ finie en chaque degré; mais on sait également définir la K-théorie continue d'un espace profini. (Voir la section 1.6.)

Les propriétés (1) et (2) dépendent essentiellement de la dégénérescence de la suite spectrale d'Atiyah-Hirzebruch associée au spectre MU et à un espace profini dont la cohomologie continue à coefficients $p$-adiques est sans torsion (espace profini sans $p$-torsion). Une fois celles-ci établies, la propriété (3) est de nature formelle. 
La démonstration du théorème 0.1 utilise de façon cruciale comme [DL] les propriétés du foncteur $\mathrm{T}$ de Lannes et son interprétation géométrique. Elle repose également sur les propriétés suivantes du spectre MU :

- La cohomologie à coefficients $p$-adiques des espaces formant le $\Omega$-spectre associé à $\mathrm{MU}$ est sans torsion en chaque degré $([\mathbf{W i}])$.

- La MU-résolution cosimpliciale canonique d'un espace profini sans p-torsion induit un diagramme simplicial augmenté acyclique en cohomologie modulo $p$ (voir le corollaire 4.2.2).

Cette dernière propriété n'est plus vraie si on remplace la MU-cohomologie par la Kthéorie. Elle vient de l'existence d'un morphisme de spectres en anneau MU $\rightarrow \mathrm{HZ} / p$.

Nous étendons la définition des foncteurs de division (foncteur $\mathrm{T}_{W}$ en section 5.3) pour prendre en compte des espaces à la source tel que le classifiant d'un $p$-groupe cyclique. La cohomologie à coefficients $p$-adiques de $\mathrm{B} \mathbb{Z} / p^{n}$ n'est pas sans torsion mais on dispose cependant d'une formule de Künneth en MU-cohomologie ([Lan]). On en déduit une expression de $\mathrm{T}_{\mathrm{BZ} / p^{n}}$ comme foncteur adjoint du produit tensoriel par $\widehat{\mathrm{MU}}^{*} \mathrm{~B} \mathbb{Z} / p^{n}$ (corollaire 8.9).

Le théorème 0.1 est un cas particulier du théorème suivant (section 7 ) :

THÉORÈme 0.2. - Soient $\pi$ un groupe de Lie compact commutatif et $X$ un espace profini fibrant dont la cohomologie continue à coefficients p-adiques est sans torsion; alors on a un isomorphisme

$$
\mathrm{T}_{\mathrm{B} \pi} \widehat{\mathrm{MU}}^{*} X \cong \widehat{\mathrm{MU}}^{*} \operatorname{hom}(\mathrm{B} \pi, X) .
$$

On en déduit un calcul des classes d'homotopie d'applications de $\mathrm{B} \pi$ dans l'espace sous-jacent à $X$ (comparer avec la proposition 6.8 ou le théorème 7.20 de $[\mathbf{D L}])$ :

Corollaire 0.3. - Soient $\pi$ un groupe de Lie compact commutatif et $X$ un espace profini fibrant dont la cohomologie continue à coefficients p-adiques est sans torsion; alors l'application

$$
[\mathrm{B} \pi,|X|] \longrightarrow \operatorname{Hom}_{\mathcal{K}_{\mathrm{MU}}}\left(\widehat{\mathrm{MU}}^{*} X, \widehat{\mathrm{MU}}^{*} \mathrm{~B} \pi\right)
$$

est une bijection.

Voici le plan de ce mémoire :

Les sections 1 à 5 exposent la théorie de l'homotopie et l'algèbre nécessaires pour obtenir les foncteurs de division en MU-cohomologie associés aux espaces fonctionnels $\operatorname{hom}(W, X)$.

La section 4 est un peu à part. On y étudie d'une part l'exactitude du produit tensoriel dans $\widehat{\mathcal{M}}$ et la commutation de ce produit tensoriel aux limites indexées par $\mathbb{N}$, d'autre part la résolution des espaces profinis par des espaces profinis sans $p$-torsion et les suites spectrales associées dans l'esprit de [CS] et [Ad1]. 
Les sections 6,7 et 8 se spécialisent au cas où $W$ est le classifiant d'un groupe de Lie compact commutatif.

Un long appendice expose les résultats sur les monades et algèbres sur une monade dont nous faisons constamment usage.

Les sections 1 à 5 et l'appendice $\mathrm{A}$ commencent par un résumé des notions et résultats exposés. Un résumé des sections 6,7 et 8 se trouve au début du chapitre 2 .

Ce mémoire fait suite à $[\mathbf{D L}]$ et reprend les résultats de ma thèse. Qu'il me soit permis de remercier Jean Lannes pour son encadrement constant. 



\section{CHAPITRE 1}

\section{COBORDISME COMPLEXE DES ESPACES PROFINIS}

Nous appelons espace un ensemble simplicial ( $c f$. [BK, Chap. VIII]). Les espaces et leurs morphismes forment une catégorie qu'on note $\mathcal{S}$. Nous fixons par ailleurs un nombre premier $p$.

\section{Théorie homotopique des espaces profinis}

Cette section décrit le cadre homotopique (celui des espaces profinis) et certains invariants algébriques que nous utilisons par la suite.

Nous considérons les catégories $\mathcal{S}$ des ensembles simpliciaux et $\widehat{\mathcal{S}}$ des ensembles profinis simpliciaux (ou espaces profinis). La catégorie $\widehat{\mathcal{S}}$ est munie d'une structure de catégorie de modèles fermée au sens de Quillen où les équivalences faibles sont les applications simpliciales continues induisant un isomorphisme en cohomologie modulo $p$ continue et où les cofibrations sont les applications (simpliciales continues) injectives degré par degré ([Mo2] $)$.

Nous rappelons dans le paragraphe 1.1 la construction d'une résolution fibrante d'un espace profini comme pro- $p$-complétion. Elle associe à tout espace profini un diagramme filtrant d'ensembles finis simpliciaux fibrants dans $\widehat{\mathcal{S}}$ dont la limite est encore fibrante. (La composée du foncteur pro-p-complétion avec la complétion profinie d'un ensemble simplicial est une version rigide de la $p$-complétion profinie d'ArtinMazur et Sullivan.) Le point essentiel est qu'une fibration principale sous l'action d'un pro- $p$-groupe simplicial est une fibration dans $\widehat{\mathcal{S}}([\mathbf{M o 2}])$.

Dans le paragraphe 1.2 nous introduisons les objets fonctionnels $\operatorname{hom}(X, Y) \in \mathcal{S}$ et $\operatorname{hom}(W, Y) \in \widehat{\mathcal{S}}$ pour $W \in \mathcal{S}$ et $X, Y \in \widehat{\mathcal{S}}$, et leurs versions pointées, qui font de $\widehat{\mathcal{S}}$ une catégorie de modèles fermée simpliciale. L'espace profini fonctionnel $\operatorname{hom}(W, Y)$ vient avec une application d'évaluation $W \widehat{\times} \operatorname{hom}(W, Y) \rightarrow Y$ où, pour tout espace profini $X$, le produit mixte $W \widehat{\times} X$ est défini comme la colimite dans $\widehat{\mathcal{S}}$ des produits 
$W_{\alpha} \times X, W_{\alpha}$ décrivant les sous-ensembles finis simpliciaux de $W$. (Nous déterminerons la cohomologie modulo $p$ continue de $W \widehat{\times} X$ en section 5.1.)

Le paragraphe 1.3 décrit les propriétés homotopiques des objets fonctionnels introduits en 1.2, principalement le fait que si $Y$ est fibrant dans $\widehat{\mathcal{S}}$ alors :

- l'application d'évaluation $W \widehat{\times} \operatorname{hom}(W, Y) \rightarrow Y$ induit une bijection entre les ensembles $\operatorname{Hom}_{\mathrm{h} \widehat{\mathcal{S}}}(W \widehat{\times} X, Y)$ et $\operatorname{Hom}_{\mathrm{h} \widehat{\mathcal{S}}}(X, \operatorname{hom}(W, Y))$, où h $\widehat{\mathcal{S}}$ désigne la catégorie homotopique associée à $\widehat{\mathcal{S}}$.

- L'ensemble des classes d'homotopie d'applications du point dans l'ensemble simplicial sous-jacent à $Y$ est naturellement en bijection avec l'ensemble des morphismes de $\mathbb{F}_{p}$-algèbres de $\mathrm{H}^{0} Y$ dans $\mathbb{F}_{p}$.

Nous comparons également, pour $X(-)$ et $Y(-)$ des diagrammes filtrants d'ensembles finis simpliciaux pointés de limite $X$ et $Y$ dans $\widehat{\mathcal{S}}_{\mathrm{pt}}$, l'ensemble des morphismes dans h $\widehat{\mathcal{S}}_{\text {pt }}$ entre $X$ et $Y$ avec l'ensemble des morphismes de pro-objets de $\mathrm{h} \mathcal{S}_{\text {pt }}$ entre $X(-)$ et $Y(-)$.

Dans le paragraphe 1.4 nous discutons brièvement de la filtration squelettale d'un espace profini puis nous revisitons quelques limites et colimites homotopiques classiques et les outils algébriques associés : les tours de fibrations et la suite exacte de Milnor, les produits fibrés homotopiques et la suite exacte longue en homotopie, les sommes amalgamées homotopiques et la suite exacte de Mayer-Vietoris en cohomologie continue.

Le paragraphe 1.5 est consacré aux espaces profinis munis d'une action principale d'un pro-p-groupe simplicial $G$. Un tel espace peut être vu comme la fibre homotopique d'un morphisme de but le classifiant de $G$. On associe à un tel morphisme une suite spectrale de Serre dont le terme $\mathrm{E}_{2}$ est donné par la cohomologie continue du groupe $G$ à coefficients dans le $\pi_{0} G$-module formé par la cohomologie continue de la fibre au sens de $[\mathbf{Q u 2}]$.

Dans le dernier paragraphe (1.6) nous associons à chaque théorie cohomologique sur $\mathcal{S}$ représentée par un $\Omega$-spectre $\left(M_{n}\right)$ une théorie cohomologique sur h $\widehat{\mathcal{S}}$ représentée par un $\Omega$-spectre $\left(\widehat{M}_{n}\right)$, sous certaines hypothèses de finitude sur $M$ : nous demandons que les espaces de lacets infinis $M_{n}$ formant le spectre $M$ soient, pour $n$ assez grand, connexes et de cohomologie modulo $p$ finie en chaque degré. L'espace profini $\widehat{M}_{n}$ est alors le $p$-complété profini de $M_{n}$ pour $n$ assez grand. Nous traduisons les résultats du paragraphe 1.4 : la suite exacte de Milnor en $M$-cohomologie continue associée à la filtration squelettale d'un espace profini, la suite exacte longue de Mayer-Vietoris associée à une somme amalgamée homotopique.

1.1. Rappel sur la $p$-complétion profinie (d'après [Mo2]). - Un pro-objet dans une catégorie $\mathcal{C}$ est un foncteur d'une catégorie petite et filtrante dans $\mathcal{C}$. Un morphisme entre deux pro-objets $X(-)$ et $Y(-)$ est un élément de l'ensemble

$$
\lim _{j} \operatorname{colim}_{i} \operatorname{Hom}_{\mathcal{C}}(X(i), Y(j)) .
$$


Les pro-objets et leurs morphismes forment une catégorie pro-C (voir par exemple [AM, Appendice, $\S 3]$ ).

Le foncteur de la catégorie des ensembles profinis et applications continues dans celle des pro-ensembles finis, qui associe à un ensemble profini $S$ le pro-objet formé des quotients finis de $S$ est une équivalence de catégories d'inverse le foncteur limite.

On note $\widehat{\mathcal{S}}$ la catégorie des ensembles profinis simpliciaux (ou espaces profinis) et des applications simpliciales continues. La sous-catégorie pleine $\mathcal{S}_{\mathrm{f}}$ de $\mathcal{S}$ formée des ensembles simpliciaux finis en chaque degré est aussi une sous-catégorie pleine de $\widehat{\mathcal{S}}$. Tout espace profini est la limite filtrante dans $\widehat{\mathcal{S}}$ de ses quotients finis simpliciaux $(c f \text {. [Qu2, lemma 2.3] })^{(1)}$. L'oubli de la topologie $\widehat{\mathcal{S}} \rightarrow \mathcal{S}$ admet un adjoint à gauche - le foncteur qui associe à un ensemble simplicial $W$ la limite du diagramme formé des quotients finis simpliciaux de $W$ - qu'on appelle complétion profinie et qu'on note $W \mapsto \widehat{W}^{f}$. Par adjonction, le complété profini d'un ensemble simplicial $W$ s'interprète comme la colimite filtrante dans $\widehat{\mathcal{S}}$ des sous-ensembles finis simpliciaux de $W$.

Soit $X$ un espace profini. On définit la cohomologie continue de $X$ à coefficients dans un groupe abélien profini $\pi$ comme l'homologie du complexe de cochaînes continues de $X$ à valeurs dans $\pi$. On la note $\mathrm{H} \pi^{*} X$ ou, si $\pi$ est le groupe $\mathbb{Z} / p, \mathrm{H}^{*} X$. Par construction, la cohomologie continue du complété profini d'un espace s'identifie à la cohomologie ordinaire de cet espace.

Si $\pi$ est fini la cohomologie continue $\mathrm{H} \pi^{*} X$ est la colimite des cohomologies ordinaires des quotients finis simpliciaux de $X$ à coefficients dans $\pi$. On a plus généralement (par commutation des limites entre elles) le

Lemme 1.1.1. - Soient $\pi$ un groupe abélien fini et $\left(X_{\alpha}\right)$ un diagramme filtrant d'espaces profinis, alors l'application $\operatorname{colim}_{\alpha} \mathrm{H} \pi^{*} X_{\alpha} \rightarrow \mathrm{H} \pi^{*} \lim _{\alpha} X_{\alpha}$ est un isomorphisme.

On déduit également de la formule de Künneth classique pour la cohomologie modulo $p$ du produit de deux ensembles finis simpliciaux le

Lemme 1.1.2. - Soient $X$ et $Y$ deux espaces profinis, alors l'application $\mathrm{H}^{*} X \otimes \mathrm{H}^{*} Y$ $\rightarrow \mathrm{H}^{*} X \times Y$ est un isomorphisme.

La catégorie $\widehat{\mathcal{S}}$ possède une structure de catégorie de modèles fermée (voir par exemple $[\mathbf{D w} \mathbf{S}]$ pour cette notion) avec pour cofibrations les applications simpliciales injectives degré par degré et pour équivalences faibles les applications induisant un isomorphisme en cohomologie modulo $p$ continue ([Mo2, théorème 1]). Tout objet de $\widehat{\mathcal{S}}$ est cofibrant. Les objets fibrants sont par définition les espaces profinis $X$ tels que l'application de $X$ dans le point a la propriété de relèvement à droite par rapport aux cofibrations qui sont des équivalences faibles. On note h $\widehat{\mathcal{S}}$ la catégorie homotopique associée.

$\overline{{ }^{(1)} \text { Dan Isacksen }}$ '’a indiqué que les catégories $\widehat{\mathcal{S}}$ et pro- $\mathcal{S}_{\mathrm{f}}$ ne sont cependant pas équivalentes. 
Résolution fibrante. - On dit qu'une application $Y \rightarrow X$ entre espaces profinis est une fibration principale sous l'action d'un pro-p-groupe simplicial $G$ si $Y$ est muni d'une action (continue) de $G$ libre degré par degré et si l'application $Y \rightarrow X$ est isomorphe à la projection $Y \rightarrow Y / G$. La proposition suivante est essentielle :

Proposition 1.1.3 ([Mo2]). - Soient $X$ et $Y$ deux espaces profinis et $Y \rightarrow X$ une fibration principale sous l'action d'un pro-p-groupe simplicial, alors $Y \rightarrow X$ est une fibration dans $\widehat{\mathcal{S}}$.

Soit $W$ un ensemble simplicial. On note Res ${ }^{\bullet}$ sa $\mathbb{F}_{p}$-résolution cosimpliciale, $\operatorname{Tot}_{s} \operatorname{Res}{ }^{\bullet} W$ le $s$-ième espace total partiel associé et $\mathrm{P}^{t} \operatorname{Tot}_{s} \operatorname{Res}^{\bullet}(W)$ la $t$-troncature de Postnikov de l'espace $\operatorname{Tot}_{s} \operatorname{Res}^{\bullet}(W)$ ([BK, part I $]$ ).

Soient $X$ un espace profini. On note, pour tout entier $s, \mathrm{R}_{s} X$ l'espace profini

$$
\lim _{Q} \operatorname{Tot}_{s} \operatorname{Res}^{\bullet}(X / Q),
$$

$Q$ décrivant l'ensemble des relations d'équivalence simpliciales ouvertes de $X$, et $\mathrm{R} X$ la limite sur $s$ des $\mathrm{R}_{s} X$. Posons $\mathrm{R}_{-1} X$ comme l'espace profini réduit au point. Les applications $\mathrm{R}_{s} X \rightarrow \mathrm{R}_{s-1} X$ sont des fibrations principales sous l'action de $\mathbb{F}_{p}$-espaces vectoriels profinis simpliciaux. En particulier les espaces profinis $\mathrm{R}_{s} X$ et leur limite $\mathrm{R} X$ sont fibrants ( $c f .[\mathrm{Mo2}, \S 2.1])$. Pour chaque quotient fini simplicial $X / Q$, l'application $X / Q \rightarrow \operatorname{Tot}_{s} \operatorname{Res}^{\bullet}(X / Q)$ induit un isomorphisme

$$
\operatorname{colim}_{s} \mathrm{H}^{*} \operatorname{Tot}_{s} \operatorname{Res}^{\bullet}(X / Q) \longrightarrow \mathrm{H}^{*} X / Q
$$

([BK, chap. III, §6]). On en déduit que l'application $X \rightarrow \mathrm{R} X$ est une équivalence faible. L'espace profini RX est donc une résolution fibrante fonctorielle de $X$ dans $\widehat{\mathcal{S}}$.

On note $\widehat{X}(-)$ le pro-objet formé des espaces $\mathrm{P}^{t} \operatorname{Tot}_{s} \operatorname{Res}^{\bullet}(X / Q)$. Chacun de ces espaces est $p$-fini, c'est à dire est un ensemble fini simplicial fibrant dans $\mathcal{S}$ dont les groupes d'homotopie sont, pour tout choix du point base, des $p$-groupes finis, triviaux sauf pour un nombre fini d'entre eux. Chacun de ces espaces est également fibrant dans $\widehat{\mathcal{S}}$ pour la même raison que les espaces profinis $\mathrm{R}_{s} X$. Les applications $X \rightarrow \widehat{X}(i)$ induisent un isomorphisme $\mathrm{R} X \rightarrow \lim _{i} \widehat{X}(i)$. On appelle $\widehat{X}(-)$ le pro-p-complété de $X$.

Soit $X$ un ensemble simplicial. On note encore $\widehat{X}(-)$ le pro-objet formé des espaces $\mathrm{P}^{t} \operatorname{Tot}_{s} \operatorname{Res}^{\bullet}(X / Q), X / Q$ décrivant les quotients finis simpliciaux de $X$, et $\widehat{X}^{p}$ la limite de $\widehat{X}(-)$ dans $\widehat{\mathcal{S}}$. On dispose d'un morphisme du pro-objet constant $X$ dans $\widehat{X}(-)$. Puisque le morphisme $\operatorname{colim}_{Q} \mathrm{H}^{*} X / Q \rightarrow \mathrm{H}^{*} X$ est un isomorphisme, il en est de même du morphisme $\mathrm{H}^{*} \widehat{X}^{p} \rightarrow \mathrm{H}^{*} X$. L'ensemble simplicial sous-jacent à $\widehat{X}^{p}$ est isomorphe dans hS au $p$-complété de Sullivan de $X$. On appelle $\widehat{X}^{p}$ le $p$-complété profini de $X$.

Un rétract d'un espace profini fibrant est fibrant. Inversement on a :

Lemme 1.1.4. - Soit $X$ un espace profini fibrant, alors $X$ est rétract de $\mathrm{R} X$.

Démonstration. - Pour chaque quotient fini simplicial $X / Q$ de $X$ l'application $X / Q$

$\rightarrow$ Tot $\operatorname{Res}^{\bullet}(X / Q)$ est injective degré par degré donc l'application $X \rightarrow \mathrm{R} X$ est une 
cofibration. Comme c'est également une équivalence faible, la propriété de relèvement à droite appliquée à la fibration $X \rightarrow$ pt donne l'existence d'un rétract.

Comme la catégorie $\mathcal{S}$, la catégorie $\widehat{\mathcal{S}}$ possède un analogue pointé $\widehat{\mathcal{S}}_{\mathrm{pt}}$. Un objet de $\widehat{\mathcal{S}}_{\text {pt }}$ est la donnée d'un morphisme pt $\rightarrow X$ dans $\widehat{\mathcal{S}}$, où pt est l'ensemble simplicial constant égal au point. Un morphisme de $\widehat{\mathcal{S}}_{\text {pt }}$ est un morphisme de $\widehat{\mathcal{S}}$ respectant le point base. Observons qu'un espace profini non vide peut toujours être pointé et que l'oubli du point base $\widehat{\mathcal{S}}_{\mathrm{pt}} \rightarrow \widehat{\mathcal{S}}$ admet un adjoint à gauche : le foncteur qui associe à un espace profini $X$ la réunion disjointe de $X$ et d'un point base, qu'on note $X_{+}$. Observons également que le complété profini d'un espace pointé est naturellement pointé et que le foncteur $X \mapsto X_{+}$est compatible avec la complétion profinie des espaces. On note $\mathrm{h} \widehat{\mathcal{S}}_{\mathrm{pt}}$ la catégorie homotopique associée à $\widehat{\mathcal{S}}_{\mathrm{pt}}$. Par construction l'ensemble $\operatorname{Hom}_{\mathrm{h} \widehat{\mathcal{S}}_{\mathrm{pt}}}\left(X_{+}, Y\right)$ s'identifie à l'ensemble $\operatorname{Hom}_{\mathrm{h} \widehat{\mathcal{S}}}(X, Y)$ pour tout $X \in \widehat{\mathcal{S}}$ et $Y \in \widehat{\mathcal{S}}_{\mathrm{pt}}$.

1.2. Objets fonctionnels. - Soit $W$ un ensemble fini simplicial. Rappelons que l'objet fonctionnel $\mathcal{S} \rightarrow \mathcal{S}, Y \mapsto \operatorname{hom}(W, Y)$ est l'adjoint à droite du foncteur $Z \mapsto$ $W \times Z$.

Notons $\mathrm{Sk}_{s} W$ le sous-ensemble simplicial de $W$ engendré par les simplexes de degré inférieur ou égal à $s$. Lorsque $Y$ est profini, l'espace fonctionnel hom $(W, Y)$, comme limite des espaces hom $\left(\operatorname{Sk}_{s} W, Y\right)$, est naturellement profini et comme tel l'adjoint à droite dans $\widehat{\mathcal{S}}$ en $Y$ du foncteur $Z \mapsto W \times Z$. On a donc une bijection naturelle

$$
\operatorname{Hom}_{\widehat{\mathcal{S}}}(W \times Z, Y) \simeq \operatorname{Hom}_{\widehat{\mathcal{S}}}(Z, \operatorname{hom}(W, Y)) .
$$

En particulier notons $\triangle[n]$ le simplexe standard de dimension $n$; c'est un ensemble fini simplicial. L'ensemble profini des $n$-simplexes de $\operatorname{hom}(W, Y)$ s'identifie à l'ensemble profini $\operatorname{Hom}_{\widehat{\mathcal{S}}}(W \times \triangle[n], Y)$ et la structure simpliciale de $\operatorname{hom}(W, Y)$ est induite par la structure cosimplicial de l'objet $[n] \mapsto \triangle[n]$.

Soit maintenant $X$ un espace profini. Il existe un ensemble simplicial $\operatorname{hom}(X, Y)$ caractérisé par la bijection

$$
\operatorname{Hom}_{\widehat{\mathcal{S}}}(X \times W, Y) \simeq \operatorname{Hom}_{\mathcal{S}}(W, \operatorname{hom}(X, Y))
$$

naturelle en $W \in \mathcal{S}_{\mathrm{f}}$ et $X, Y \in \widehat{\mathcal{S}}$. L'application d'évaluation $W \times \operatorname{hom}(W, Y) \rightarrow Y$ dans $\widehat{\mathcal{S}}$ induit alors un isomorphisme

$$
\operatorname{hom}(W \times X, Y) \stackrel{\sim}{\longleftarrow} \operatorname{hom}(X, \operatorname{hom}(W, Y))
$$

entre ensembles simpliciaux.

L'objet fonctionnel $X, Y \mapsto \operatorname{hom}(X, Y)$ fait de $\widehat{\mathcal{S}}$ une catégorie de modèles fermée simpliciale ( $c f$. [Qu1, II, $\S 1,2]$ ) : Le fait que pour toute cofibration $X \rightarrow X^{\prime}$ et pour toute fibration $Y \rightarrow Y^{\prime}$ dans $\widehat{\mathcal{S}}$ l'application

$$
\operatorname{hom}\left(X^{\prime}, Y\right) \longrightarrow \operatorname{hom}(X, Y) \times \operatorname{hom}\left(X, Y^{\prime}\right) \operatorname{hom}\left(X^{\prime}, Y^{\prime}\right)
$$

est une fibration de $\mathcal{S}$ et une équivalence faible si de plus $X \rightarrow X^{\prime}$ ou $Y \rightarrow Y^{\prime}$ l'est dans $\widehat{\mathcal{S}}$ vient par adjonction de ce que pour toute application injective $W \rightarrow W^{\prime}$ 
entre ensembles finis simpliciaux (plus généralement pour toute cofibration $W \rightarrow W^{\prime}$ dans $\widehat{\mathcal{S}}$ ) l'application

$$
\left(X \times W^{\prime}\right) \cup_{X \times W}\left(X^{\prime} \times W\right) \longrightarrow X^{\prime} \times W^{\prime}
$$

est une cofibration de $\widehat{\mathcal{S}}$ qui est une équivalence faible si $W \rightarrow W^{\prime}$ ou $X \rightarrow X^{\prime}$ l'est. Ce dernier point s'obtient par la formule de Künneth en cohomologie modulo $p$ continue (lemme 1.1.2) et en appliquant la suite exacte longue de Mayer-Vietoris.

En particulier pour $X$ et $Y$ dans $\widehat{\mathcal{S}}$, l'ensemble $\operatorname{simplicial} \operatorname{hom}(X, Y)$ est fibrant dès que $Y$ est fibrant.

Lorsque $W, X$ et $Y$ sont pointés, les objets fonctionnels $\operatorname{hom}(W, Y) \in \widehat{\mathcal{S}}$ et $\operatorname{hom}(X, Y) \in \mathcal{S}$ admettent des versions pointées : Notons, pour $Z$ un espace profini pointé, $X \vee Z$ la somme de $X$ et de $Z$ au dessous du point et $X \wedge Z$ le quotient $(W \times Z) /(W \vee Z)($ dans $\widehat{\mathcal{S}})$. Les objets fonctionnels $\operatorname{hom}_{\mathrm{pt}}(W, Y) \in \widehat{\mathcal{S}}_{\mathrm{pt}}$ et $\operatorname{hom}_{\mathrm{pt}}(X, Y) \in \mathcal{S}_{\mathrm{pt}}$ sont caractérisés par les bijections naturelles

$$
\operatorname{Hom}_{\widehat{\mathcal{S}}_{\mathrm{pt}}}(W \wedge Z, Y) \simeq \operatorname{Hom}_{\widehat{\mathcal{S}}_{\mathrm{pt}}}\left(Z, \operatorname{hom}_{\mathrm{pt}}(W, Y)\right)
$$

et

$$
\operatorname{Hom}_{\widehat{\mathcal{S}}_{\mathrm{pt}}}(X \wedge W, Y) \simeq \operatorname{Hom}_{\mathcal{S}_{\mathrm{pt}}}\left(W, \operatorname{hom}_{\mathrm{pt}}(X, Y)\right) .
$$

L'application d'évaluation $W \wedge \operatorname{hom}_{\mathrm{pt}}(W, Y) \rightarrow Y$ induit à nouveau un isomorphisme dans $\mathcal{S}$

$$
\operatorname{hom}_{\mathrm{pt}}(W \wedge X, Y) \stackrel{\sim}{\longleftarrow} \operatorname{hom}_{\mathrm{pt}}\left(X, \operatorname{hom}_{\mathrm{pt}}(W, Y)\right) .
$$

Comme $\widehat{\mathcal{S}}$ la catégorie $\widehat{\mathcal{S}}_{\mathrm{pt}}$ est une catégorie de modèles fermée simpliciale. En particulier pour tout paire $X, Y$ d'espaces profinis pointés l'ensemble simplicial $\operatorname{hom}_{\mathrm{pt}}(X, Y)$ est fibrant si $Y$ est fibrant.

L'adjonction entre $\widehat{\mathcal{S}}$ et $\widehat{\mathcal{S}}_{\text {pt }}$ induit, pour tout espace profini $X$, un isomorphisme canonique $\operatorname{hom}_{\mathrm{pt}}\left(X_{+}, Y\right) \simeq \operatorname{hom}(X, Y)$ entre ensembles simpliciaux pointés (entre ensembles profinis simpliciaux pointés si $X$ est un ensemble fini simplicial), le second étant pointé par la composée $X \rightarrow$ pt $\rightarrow Y$. Via cet isomorphisme, les énoncés dans $\widehat{\mathcal{S}}_{\mathrm{pt}}$ qui suivent ont une version non pointée que nous sous-entendrons le plus souvent.

Parce que $W$ est fini en chaque degré, le foncteur $\widehat{\mathcal{S}}_{\mathrm{pt}} \rightarrow \widehat{\mathcal{S}}_{\mathrm{pt}}, X \mapsto W \wedge X$ commute aux colimites. Par adjonction le foncteur $\widehat{\mathcal{S}}_{\mathrm{pt}} \rightarrow \mathcal{S}_{\mathrm{pt}}, X \mapsto \operatorname{hom}_{\mathrm{pt}}(X, Y)$ transforme les colimites en limites.

Cas particulier. - Notons $\mathrm{S}$ le quotient du simplexe standard $\triangle[1]$ de dimension 1 par son bord ; c'est un ensemble fini simplicial équivalent faiblement au groupe abélien simplicial $K(\mathbb{Z}, 1)$ mais qui n'est pas fibrant dans $\mathcal{S}$ (le groupe fondamental de $\mathrm{K}(\mathbb{Z}, 1)$ est infini). Pour $X$ et $Y$ des espaces profinis pointés, on note $\Sigma X$ l'espace profini pointé $\mathrm{S} \wedge X$ (la suspension de $X$ ) et $\Omega Y$ l'espace profini pointé $\operatorname{hom}_{\mathrm{pt}}(\mathrm{S}, Y)$ (l'espace profini de lacets de $Y)$. On a donc un isomorphisme naturel $\operatorname{hom}_{\mathrm{pt}}(\Sigma X, Y) \simeq \operatorname{hom}_{\mathrm{pt}}(X, \Omega Y)$.

Soient à nouveau $X$ et $Y$ deux espaces profinis pointés et soit $W$ un ensemble simplicial pointé quelconque qu'on écrit comme la colimite filtrante de ses sous-ensembles 
finis simpliciaux pointés $W_{\alpha}$. On définit l'espace profini pointé hom pt $(W, Y)$ comme la limite dans $\widehat{\mathcal{S}}_{\mathrm{pt}}$ des espaces profinis $\operatorname{hom}_{\mathrm{pt}}\left(W_{\alpha}, Y\right)$. Dualement on définit l'espace profini pointé $W \widehat{\wedge} X$ (respectivement $X \widehat{\wedge} W$ ) comme la colimite dans $\widehat{\mathcal{S}}_{\mathrm{pt}}$ des espaces profinis $W_{\alpha} \wedge X$ (respectivement $X \wedge W_{\alpha}$ ). Les bijections qui précèdent pour $W$ fini simplicial induisent par passage à la limite des bijections

$$
\operatorname{Hom}_{\widehat{\mathcal{S}}_{\mathrm{pt}}}(W \widehat{\wedge} X, Y) \stackrel{\sim}{\longleftarrow} \operatorname{Hom}_{\widehat{\mathcal{S}}_{\mathrm{pt}}}\left(X, \operatorname{hom}_{\mathrm{pt}}(W, Y)\right)
$$

et

$$
\operatorname{Hom}_{\widehat{\mathcal{S}}_{\mathrm{pt}}}(X \widehat{\wedge} W, Y) \stackrel{\sim}{\sim} \operatorname{Hom}_{\mathcal{S}_{\mathrm{pt}}}\left(W, \operatorname{hom}_{\mathrm{pt}}(X, Y)\right)
$$

correspondant à des applications d'évaluation $W \widehat{\wedge} \operatorname{hom}_{\mathrm{pt}}(W, Y) \rightarrow Y$ dans $\widehat{\mathcal{S}}_{\mathrm{pt}}$ et $X \widehat{\wedge} \operatorname{hom}_{\mathrm{pt}}(X, Y) \rightarrow Y$ dans $\mathcal{S}_{\mathrm{pt}}$, et plus généralement des isomorphismes

$$
\operatorname{hom}_{\mathrm{pt}}(W \widehat{\wedge} X, Y) \stackrel{\sim}{\sim} \operatorname{hom}_{\mathrm{pt}}\left(X, \operatorname{hom}_{\mathrm{pt}}(W, Y)\right)
$$

et

$$
\operatorname{hom}_{\mathrm{pt}}(X \widehat{\wedge} W, Y) \stackrel{\sim}{\sim} \operatorname{hom}_{\mathrm{pt}}\left(W, \operatorname{hom}_{\mathrm{pt}}(X, Y)\right)
$$

dans $\mathcal{S}_{\mathrm{pt}}$ naturels en $W \in \mathcal{S}_{\mathrm{pt}}$ et $X, Y \in \widehat{\mathcal{S}}_{\mathrm{pt}}$.

Pour $W$ et $X$ dans $\mathcal{S}$ et $\widehat{\mathcal{S}}$ respectivement nous notons $W \widehat{\times} X$ la colimite des espaces profinis $W_{\alpha} \times X$ dans $\widehat{\mathcal{S}}$ de sorte que l'espace profini pointé $(W \widehat{\times} X)_{+}$s'identifie à $W_{+} \widehat{\wedge} X_{+}$.

Notons $|Y|$ l'ensemble simplicial sous-jacent à un espace profini $Y$. (Le foncteur $Y \mapsto|Y|$ est l'adjoint à droite de la complétion profinie $W \mapsto \widehat{W}^{f}$.) Les applications $W_{\alpha} \rightarrow \widehat{W}^{f}$ induisent une application $W \widehat{\wedge} X \rightarrow \widehat{W}^{f} \wedge X$ qui est un isomorphisme dans $\widehat{\mathcal{S}}_{\mathrm{pt}}$ si $X$ est fini simplicial, d'où un isomorphisme

$$
\operatorname{hom}_{\mathrm{pt}}\left(\widehat{W}^{f}, Y\right) \simeq\left|\operatorname{hom}_{\mathrm{pt}}(W, Y)\right|=\operatorname{hom}_{\mathrm{pt}}(W,|Y|)
$$

dans $\mathcal{S}_{\mathrm{pt}}$ donnant une structure naturelle d'espace profini à l'espace $\operatorname{hom}_{\mathrm{pt}}\left(\widehat{W}^{f}, Y\right)$.

LEMme 1.2.1. - Soient $W$ un ensemble simplicial pointé et $Y$ un espace profini pointé fibrant; alors l'espace profini $\operatorname{hom}_{\mathrm{pt}}(W, Y)$ est fibrant.

Démonstration. - On observe d'abord que les applications $\operatorname{hom}_{\mathrm{pt}}\left(W, \mathrm{R}_{s} Y\right) \rightarrow$ $\operatorname{hom}_{\mathrm{pt}}\left(W, \mathrm{R}_{s-1} Y\right)$ sont des fibrations principales sous l'action de $\mathbb{F}_{p}$-espaces vectoriels profinis simpliciaux donc que la limite $\operatorname{hom}_{\mathrm{pt}}(W, \mathrm{R} Y)$ est fibrante. On utilise ensuite le fait que $Y$ est rétract de $\mathrm{R} Y$ (lemme 1.1.4) donc $\operatorname{hom}_{\mathrm{pt}}(W, Y)$ est rétract de $\operatorname{hom}_{\mathrm{pt}}(W, \mathrm{R} Y)$ donc est fibrant.

1.3. Groupes d'homotopie. - Soit $X$ un ensemble simplicial. Rappelons qu'on définit l'ensemble $\pi_{0} X$ comme le coégalisateur des applications $\mathrm{d}_{0}, \mathrm{~d}_{1}: X_{1} \rightrightarrows X_{0}$. Pour tout espace $Y$ l'ensemble $\pi_{0} \operatorname{hom}(X, Y)$ est par définition l'ensemble des classes d'homotopie simpliciale d'applications de $X$ dans $Y$. Lorsque $Y$ est fibrant celui-ci s'identifie à l'ensemble $\operatorname{Hom}_{\mathrm{h} \mathcal{S}}(X, Y)([\mathbf{Q u 1}])$. De même, si $X$ et $Y$ sont pointés et $Y$ est fibrant alors $\pi_{0} \operatorname{hom}_{\mathrm{pt}}(X, Y)$ s'identifie à $\operatorname{Hom}_{\mathrm{h}} \mathcal{S}_{\mathrm{pt}}(X, Y)$. 
Le foncteur $X \mapsto \pi_{0} X$ jouit des propriétés suivantes :

- L'application de $X \rightarrow \pi_{0} X$ induit un isomorphisme $\left(\mathbb{F}_{p}\right)^{\pi_{0} X} \rightarrow \mathrm{H}^{0} X$.

- Notons $\operatorname{Hom}_{\mathbb{F}_{p} \text {-Alg }}\left(\mathrm{H}^{0} X, \mathbb{F}_{p}\right)$ l'ensemble (profini) des morphismes d'algèbres de $\mathrm{H}^{0} X$ dans $\mathbb{F}_{p} ;$ l'application

$$
\pi_{0} X \simeq \operatorname{Hom}_{\mathrm{h} \mathcal{S}}(\mathrm{pt}, X) \longrightarrow \operatorname{Hom}_{\mathbb{F}_{p}-\mathrm{Alg}}\left(\mathrm{H}^{0} X, \mathbb{F}_{p}\right)
$$

est une bijection si $\pi_{0} X$ est fini.

La proposition suivante ([DL, prop.1.1.3]) indique la commutation de $\pi_{0}$ avec certaines limites filtrantes :

Proposition 1.3.1. - Soit $X(-)$ un diagramme filtrant d'ensembles finis simpliciaux fibrants dans $\mathcal{S}$; alors l'application $\pi_{0} \lim _{i} X(i) \rightarrow \lim _{i} \pi_{0} X(i)$ est une bijection.

Soit maintenant $X$ un espace profini pointé. Les applications $\mathrm{d}^{0}, \mathrm{~d}^{1}: \triangle[0] \rightarrow \triangle[1]$ et $\mathrm{s}^{0}: \triangle[1] \rightarrow \triangle[0]$ induisent pour tout espace profini pointé $X$ des applications $X \vee X \rightarrow$ $X \wedge \triangle[1]_{+}$et $X \wedge \triangle[1]_{+} \rightarrow X$ qui font de $X \wedge \triangle[1]_{+}$un (bon) objet cylindre pour $X$ dans $\widehat{\mathcal{S}}_{\mathrm{pt}}$ : les applications $X \rightarrow X \wedge \triangle[1]_{+}$sont des cofibrations et des équivalences faibles et l'application $X \wedge \triangle[1]_{+} \rightarrow X$ est une équivalence faible. On en déduit que lorsque $Y$ est un espace profini fibrant les ensembles $\pi_{0} \operatorname{hom}_{\mathrm{pt}}(X, Y)$ et $\pi_{0} \operatorname{hom}(X, Y)$ s'identifient aux ensembles $\operatorname{Hom}_{\mathrm{h} \widehat{\mathcal{S}}_{\mathrm{pt}}}(X, Y)$ et $\operatorname{Hom}_{\mathrm{h} \widehat{\mathcal{S}}}(X, Y)$ respectivement. En particulier les isomorphismes entre objets fonctionnels décrits dans la section précédente donnent, si $Y$ est fibrant, des bijections naturelles

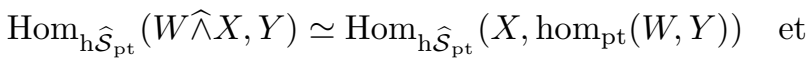

$$
\begin{aligned}
& \operatorname{Hom}_{\mathrm{h} \widehat{\mathcal{S}}_{\mathrm{pt}}}(X \widehat{\wedge} W, Y) \simeq \operatorname{Hom}_{\mathrm{h} \mathcal{S}_{\mathrm{pt}}}\left(W, \operatorname{hom}_{\mathrm{pt}}(X, Y)\right) \text { pour } W \in \mathcal{S}_{\mathrm{pt}} \text { et } X, Y \in \widehat{\mathcal{S}}_{\mathrm{pt}} \text {, } \\
& \operatorname{Hom}_{\mathrm{h} \widehat{\mathcal{S}}_{\mathrm{pt}}}\left(\widehat{W}^{f}, Y\right) \simeq \operatorname{Hom}_{\mathrm{h} \mathcal{S}_{\mathrm{pt}}}(W,|Y|) \text { pour } W \in \mathcal{S}_{\mathrm{pt}} \text { et } Y \in \widehat{\mathcal{S}}_{\mathrm{pt}}
\end{aligned}
$$

et leurs versions non pointées. (Observons que les espaces pointés (profini pour le premier $) \operatorname{hom}_{\mathrm{pt}}(W, Y), \operatorname{hom}_{\mathrm{pt}}(X, Y)$ et $|Y|$ sont fibrants dans $\mathcal{S}_{\mathrm{pt}}$ (dans $\widehat{\mathcal{S}}_{\mathrm{pt}}$ pour le premier) si $Y$ est fibrant dans $\widehat{\mathcal{S}}_{\mathrm{pt}}$.)

Les deux premières bijections montrent qu'une équivalence faible $W \rightarrow W^{\prime}$ dans $\mathcal{S}_{\mathrm{pt}}$ induit des équivalences faibles $W \widehat{\wedge} X \rightarrow W^{\prime} \widehat{\wedge} X$ et $\operatorname{hom}_{\mathrm{pt}}\left(W^{\prime}, Y\right) \rightarrow \operatorname{hom}_{\mathrm{pt}}(W, Y)$ dans $\widehat{\mathcal{S}}_{\mathrm{pt}}$ si $Y$ est fibrant. Voir aussi le corollaire 5.1.4 de la section 5 .

Cas particulier. - L'isomorphisme naturel $\operatorname{hom}_{\mathrm{pt}}(\Sigma X, Y) \simeq \operatorname{hom}_{\mathrm{pt}}(X, \Omega Y)$ induit une bijection $\operatorname{Hom}_{\mathrm{h} \widehat{\mathcal{S}}_{\mathrm{pt}}}(\Sigma X, Y) \simeq \operatorname{Hom}_{\mathrm{h} \widehat{\mathcal{S}}_{\mathrm{pt}}}(X, \Omega Y)$ pour $X, Y \in \widehat{\mathcal{S}}_{\mathrm{pt}}$ avec $Y$ fibrant. Notons $\mathrm{R}^{\prime} W$ la résolution fibrante dans $\mathcal{S}$ d'un ensemble simplicial $W$. On dispose d'une application $\mathrm{S} \rightarrow \mathrm{R}^{\prime}(\mathrm{S} \vee \mathrm{S})$ faisant de $\mathrm{S}$ un objet en cogroupe de $\mathrm{h} \mathcal{S}_{\mathrm{pt}}$. On en déduit, pour tout espace profini $X$ et tout espace profini fibrant $Y$ une structure naturelle d'objet en cogroupe sur $\Sigma X$ et d'objet en groupe sur $\Omega Y$ dans $\mathrm{h} \widehat{\mathcal{S}}_{\mathrm{pt}}$. Avec ces structures la bijection $\operatorname{Hom}_{\mathrm{h} \widehat{\mathcal{S}}_{\mathrm{pt}}}(\Sigma X, Y) \simeq \operatorname{Hom}_{\mathrm{h} \widehat{\mathcal{S}}_{\mathrm{pt}}}(X, \Omega Y)$ est un isomorphisme de groupes. 
Comparaison avec la catégorie pro-h $\mathcal{S}_{\mathrm{pt}}$. - Soient $X$ et $Y$ deux ensembles finis simpliciaux pointés. L'application $Y \rightarrow|\mathrm{R} Y|$ dans $\mathcal{S}_{\text {pt }}$ induit une application

$$
\operatorname{Hom}_{\mathrm{h} \mathcal{S}_{\mathrm{pt}}}(X, Y) \longrightarrow \operatorname{Hom}_{\mathrm{h} \mathcal{S}_{\mathrm{pt}}}(X,|\mathrm{R} Y|) \simeq \operatorname{Hom}_{\mathrm{h} \widehat{\mathcal{S}}_{\mathrm{pt}}}(X, Y) .
$$

Proposition 1.3.2. - Soient $X(-)$ un pro-ensemble fini simplicial pointé de limite $X$ dans $\widehat{\mathcal{S}}_{\mathrm{pt}}$ et $Y$ un espace $p$-fini pointé, alors l'application

$$
\operatorname{colim}_{j} \operatorname{Hom}_{\mathrm{h} \mathcal{S}_{\mathrm{pt}}}(X(j), Y) \longrightarrow \operatorname{Hom}_{\mathrm{h} \widehat{\mathcal{S}}_{\mathrm{pt}}}(X, Y)
$$

est une bijection.

Démonstration. - On commence par observer que le morphisme du pro-espace $p$ fini constant $Y$ dans le pro-p-complété $\widehat{Y}(-)$ induit un isomorphisme en cohomologie modulo $p$ continue donc est un pro-isomorphisme dans $\mathrm{h} \mathcal{S}_{\mathrm{pt}}$ ([Mo1, Théo. 2.4.1]), donc également un pro-isomorphisme dans h $\widehat{\mathcal{S}}_{\text {pt }}$ (deux applications entre ensembles finis simpliciaux fibrants dans $\mathcal{S}$ qui coïncident dans hS sont simplicialement homotopes donc coïncident dans h $\widehat{\mathcal{S}}$ ).

Pour chaque couple d'indices $(i, j)$ l'application

$$
\operatorname{Hom}_{\mathrm{h} \mathcal{S}_{\mathrm{pt}}}(X(j), \widehat{Y}(i)) \longrightarrow \operatorname{Hom}_{\mathrm{h} \widehat{\mathcal{S}}_{\mathrm{pt}}}(X(j), \widehat{Y}(i))
$$

est bijective $(\widehat{Y}(i)$ est fibrant dans $\widehat{\mathcal{S}})$. L'application

$$
\operatorname{colim}_{j} \operatorname{Hom}_{\mathrm{h} \mathcal{S}_{\mathrm{pt}}}(X(j), \widehat{Y}(i)) \longrightarrow \operatorname{Hom}_{\mathrm{h} \widehat{\mathcal{S}}_{\mathrm{pt}}}(X, \widehat{Y}(i))
$$

est bijective puisque l'application $\operatorname{colim}_{j} \operatorname{hom}_{\mathrm{pt}}(X(j), \widehat{Y}(i)) \rightarrow \operatorname{hom}_{\mathrm{pt}}(X, \widehat{Y}(i))$ est un isomorphisme dans $\mathcal{S}$. On en déduit le résultat.

Corollaire 1.3.3. - Soient $X(-)$ un pro-ensemble fini simplicial pointé et $Y(-)$ un pro-espace $p$-fini dont on note $X$ et $Y$ les limites respectives dans $\widehat{\mathcal{S}}_{\mathrm{pt}}$. On suppose que $X$ est isomorphe au complété profini d'un ensemble simplicial, alors on a une bijection naturelle

$$
\operatorname{Hom}_{\mathrm{h} \widehat{\mathcal{S}}_{\mathrm{pt}}}(X, Y) \simeq \operatorname{Hom}_{\mathrm{pro}-\mathrm{h} \mathcal{S}_{\mathrm{pt}}}(X(-), Y(-)) .
$$

Démonstration. - Puisque $X$ est isomorphe au complété profini d'un espace, les espaces $\operatorname{hom}_{\mathrm{pt}}(X, \widehat{Y}(i))$ sont naturellement (en $i$ ) limites d'ensembles finis simpliciaux fibrants dans $\mathcal{S}$ donc l'application

$$
\operatorname{Hom}_{\mathrm{h} \widehat{\mathcal{S}}_{\mathrm{pt}}}(X, Y) \simeq \operatorname{Hom}_{\mathrm{h} \widehat{\mathcal{S}}_{\mathrm{pt}}}(X, \mathrm{R} Y) \longrightarrow \lim _{i} \operatorname{Hom}_{\mathrm{h} \widehat{\mathcal{S}}_{\mathrm{pt}}}(X, \widehat{Y}(i))
$$

est bijective par la proposition 1.3.1 et l'interprétation des ensembles $\operatorname{Hom}_{\mathrm{h} \widehat{\mathcal{S}}_{\mathrm{pt}}}(-,-)$ comme $\pi_{0}$ d'espaces fonctionnels. On en déduit par la proposition ci-dessus une bijection

$$
\operatorname{Hom}_{\mathrm{h} \widehat{\mathcal{S}}_{\mathrm{pt}}}(X, Y) \simeq \operatorname{Hom}_{\mathrm{pro}-\mathrm{h} \mathcal{S}_{\mathrm{pt}}}(X(-), \widehat{Y}(-)) .
$$

On conclut en observant à nouveau que les pro-espaces $p$-finis pointés $\widehat{Y}(-)$ et $Y(-)$ sont isomorphes dans pro-h $\mathcal{S}_{\text {pt }}$ puisqu'on a des morphismes $Y(i) \rightarrow Y(i) \widehat{(-)}$ et 
$\widehat{Y}(-) \rightarrow Y(i) \widehat{(-)}$ induisant des isomorphismes $\operatorname{colim}_{i, j} \mathrm{H}^{*}\left(Y(i) \widehat{(j)} \rightarrow \operatorname{colim}_{i} \mathrm{H}^{*} Y(i)\right.$ et $\operatorname{colim}_{i, j} \mathrm{H}^{*}(Y(i) \widehat{Y}(j)) \rightarrow \operatorname{colim}_{i} \mathrm{H}^{*} \widehat{Y}(i)$.

REMARQUE. - Le corollaire n'est pas vrai sans hypothèse sur $X$, voir la section suivante pour le cas où $Y(-)$ est une tour.

$\pi_{0}$ d'un espace profini. - Soit $X$ un espace profini. On note encore $\pi_{0} X$ le coégalisateur des applications $\mathrm{d}_{0}, \mathrm{~d}_{1}: X_{1} \rightrightarrows X_{0}$ dans la catégorie $\widehat{\mathcal{E} n s}$ des ensembles profinis. Par construction le foncteur $\widehat{\mathcal{S}} \rightarrow \widehat{\mathcal{E} \text { ns }}, X \mapsto \pi_{0} X$ commute aux limites filtrantes.

Rappelons qu'on note $|X|$ l'ensemble simplicial sous-jacent à $X$.

Proposition 1.3.4. - Soit $X$ un espace profini, alors :

(a) L'application $X \rightarrow \pi_{0} X$ induit un isomorphisme entre l'ensemble des applications continues de $\pi_{0} X$ dans $\mathbb{F}_{p}$ et $\mathrm{H}^{0} X$, et une bijection $\operatorname{Hom}_{\mathrm{h} \widehat{\mathcal{S}}}(\mathrm{pt}, X) \stackrel{\sim}{\rightarrow} \pi_{0} X$.

(b) L'application d'évaluation $\pi_{0} X \rightarrow \operatorname{Hom}_{\mathbb{F}_{p}-\mathrm{Alg}}\left(\mathrm{H}^{0} X, \mathbb{F}_{p}\right)$ est un homéomorphisme.

(c) Supposons $X$ fibrant alors l'application $X \rightarrow \pi_{0} X$ induit une bijection entre $\pi_{0}|X|$ et l'ensemble sous-jacent à $\pi_{0} X$.

Démonstration. - La première partie du point (a) et le point (b) s'obtiennent en écrivant $X$ comme la limite de ses quotients finis simpliciaux. Le point (b) montre que $X \mapsto \pi_{0} X$ transforme les équivalences faibles en homéomorphismes. La deuxième partie du point (a) se ramène donc au cas où $X$ est fibrant, lequel vient de ce que $\mathrm{pt}_{+} \wedge \triangle[1]_{+}$est un objet cylindre pour pt $(c f .[\mathbf{Q u 1}])$.

Le point (c) s'obtient en considérant le diagramme

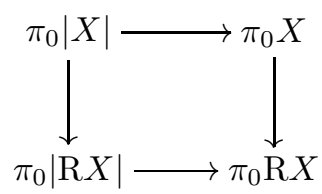

L'application $X \rightarrow \mathrm{R} X$ est une équivalence faible entre objets fibrants donc une équivalence d'homotopie simpliciale, donc induit une équivalence faible entre les ensembles simpliciaux sous-jacents. Les applications $\pi_{0} X \rightarrow \pi_{0} \mathrm{R} X$ et $\pi_{0}|X| \rightarrow \pi_{0}|\mathrm{R} X|$ sont donc des bijections. Par la proposition 1.3.1, l'application $\pi_{0}|\mathrm{R} X| \rightarrow \lim _{i} \pi_{0} \widehat{X}(i)$ est une bijection. L'application $\pi_{0} \mathrm{R} X \rightarrow \lim _{i} \pi_{0} \widehat{X}(i)$ est également une bijection. L'application $\pi_{0}|\mathrm{R} X| \rightarrow \pi_{0} \mathrm{R} X$ est donc bijective.

Remarque. - La bijection $\operatorname{Hom}_{\mathrm{h} \widehat{\mathcal{S}}}(\mathrm{pt}, X) \simeq \pi_{0} X$ munit le premier ensemble d'une structure profinie naturelle. Les points (a) et (b) montrent que l'application $\operatorname{Hom}_{\mathrm{h} \widehat{\mathcal{S}}}(\mathrm{pt}, X) \rightarrow \operatorname{Hom}_{\mathbb{F}_{p}-\mathrm{Alg}}\left(\mathrm{H}^{0} X, \mathbb{F}_{p}\right)$ est une bijection (homéomorphisme d'ensembles profinis).

Supposons $X$ pointé et fibrant et soit $k$ un entier strictement positif. On définit le $k$-ième groupe d'homotopie $\pi_{k} X$ comme le $\pi_{0}$ de l'espace de lacets itéré $\Omega^{k} X$. 
Toute équivalence faible entre espaces profinis fibrants induit une équivalence faible entre leurs espaces de lacets qui sont fibrants, donc induit un isomorphisme entre les groupes d'homotopie. Le fait que le foncteur $\pi_{0}$ transforme les équivalences faibles de $\widehat{\mathcal{S}}$ en homéomorphismes et qu'il commute aux limites filtrantes dans $\widehat{\mathcal{S}}$ (par construction) montre que le morphisme du pro-objet constant $X$ dans le pro- $p$-complété $\widehat{X}(-)$ induit pour tout entier $k$ un isomorphisme $\pi_{k} X \rightarrow \lim _{i} \pi_{k} \widehat{X}(i)$. En particulier $\pi_{k} X$ est un pro- $p$-groupe pour tout $k \geqslant 1$.

\subsection{Quelques limites et colimites homotopiques}

Filtration squelettale. - Soient $X$ un espace profini et $n$ un entier. On note $\operatorname{Sk}_{n} X$ et on appelle $n$-ième squelette de $X$ le sous-ensemble simplicial de $X$ engendré par les simplexes de degré inférieur ou égal à $n$. Pour tout entier $k$ l'ensemble des $k$ simplexes de $\mathrm{Sk}_{n} X$ est fermé dans $X_{k}$ de sorte que $\mathrm{Sk}_{n} X$ est un sous - ensemble profini simplicial de $X$.

Pour tout entier $n$ l'espace profini $\mathrm{Sk}_{n} X$ est la somme amalgamée du diagramme

$$
X_{n} \times \triangle[n] \longleftarrow X_{n} \times \mathrm{Sk}_{n-1} \triangle[n] \longrightarrow \mathrm{Sk}_{n-1} X
$$

induit par l'application évidente $X_{n} \times \triangle[n] \rightarrow X$, où $X_{n}$ est pris comme ensemble profini simplicial constant. Les morphismes $\mathrm{Sk}_{n-1} X \rightarrow \mathrm{Sk}_{n} X$ sont des cofibrations et $X$ est la colimite dans $\widehat{\mathcal{S}}$ de la suite (stationnaire degré par degré) formée par les $\mathrm{Sk}_{n} X$.

Le lemme suivant nous sera utile dans la section 8 :

Lemme 1.4.1. - Soient $X$ et $Y$ deux espaces profinis. On suppose que $X$ est de dimension finie; alors l'application

$$
\operatorname{colim}_{s} \operatorname{Hom}_{\mathrm{h} \widehat{\mathcal{S}}}\left(X, \mathrm{Sk}_{s} Y\right) \longrightarrow \operatorname{Hom}_{\mathrm{h} \widehat{\mathcal{S}}}(X, Y)
$$

est une bijection.

Démonstration. - Soit $s$ un entier et notons $Y(-)$ le diagramme des quotients finis simpliciaux de $Y$; alors le diagramme $\operatorname{Sk}_{s} Y(-)$ a pour limite $\operatorname{Sk}_{s} Y$ dans $\widehat{\mathcal{S}}$. Pour chaque indice $\alpha$ l'application $\operatorname{Sk}_{s} Y(\alpha) \rightarrow Y(\alpha)$ induit en cohomologie modulo $p$ un isomorphisme en degré inférieur strictement à $s$ donc induit une équivalence d'homotopie entre les $(s-1)$-troncatures de Postnikov des $p$-complétés de BousfieldKan de $\operatorname{Sk}_{s} Y(\alpha)$ et $Y(\alpha)$ ([BK, Ch. I, lemma 6.2]). En passant à la limite sur $\alpha$ et par construction de la résolution fibrante dans $\widehat{\mathcal{S}}$ on en déduit que l'application $\mathrm{R} \mathrm{Sk}_{s} Y \rightarrow \mathrm{R} Y$ induit une équivalence d'homotopie au niveau des $(s-1)$-troncatures de Postnikov, donc, pour tout espace profini $X$ de dimension inférieure strictement à $s$, une bijection $\pi_{0} \operatorname{hom}\left(X, \mathrm{R} \mathrm{Sk}_{s} Y\right) \rightarrow \pi_{0} \operatorname{hom}(X, \mathrm{R} Y)$. 
Limite d'une tour de fibrations. - Nous utilisons le résultat suivant ([BK, Chap. IX, thm 3.1]) :

Proposition 1.4.2. - Soit $\cdots \rightarrow X_{n} \rightarrow \cdots \rightarrow X_{-1}=$ pt une tour de fibrations entre espaces pointés, alors :

(a) Les applications $\lim _{n} X_{n} \rightarrow X_{m}, m \in \mathbb{N}$, induisent une surjection

$$
\pi_{0} \lim _{n} X_{n} \longrightarrow \lim _{n} \pi_{0} X_{n}
$$

entre ensembles pointés.

(b) L'image réciproque par cette surjection du point base de $\lim _{n} \pi_{0} X_{n}$ s'identifie à l'ensemble $\lim _{n}^{1} \pi_{1} X_{n}$

Dans le point (b), le terme $\lim _{n}^{1} G_{n}$ pour une tour de groupes $\left(G_{n}, j: G_{n+1} \rightarrow G_{n}\right)$ est défini comme l'ensemble des orbites de l'action du groupe $\prod G_{n}$ sur l'ensemble sous-jacent à $\prod G_{n}$ donné par $\left(g_{n}\right) \cdot\left(x_{n}\right)=\left(g_{n} x_{n}\left(j g_{n+1}\right)^{-1}\right)$. Cf. [BK, Chap IX, $\left.\S 2\right]$.

Corollaire 1.4.3. - Soient $\left(\cdots X_{n} \rightarrow X_{n+1} \rightarrow \cdots\right)$ une suite de cofibrations entre espaces profinis pointés, $\left(Y_{n}\right)$ une tour d'espaces profinis pointés fibrants et $\varphi$ un élément de $\lim _{n} \operatorname{Hom}_{\mathrm{h} \widehat{\mathcal{S}}_{\mathrm{pt}}}\left(X_{n}, Y_{n}\right)$ dont on note $\varphi_{n}$ la projection sur $\operatorname{Hom}_{\mathrm{h} \widehat{\mathcal{S}}_{\mathrm{pt}}}\left(X_{n}, Y_{n}\right)$. Alors l'application

$$
\operatorname{Hom}_{\mathrm{h} \widehat{\mathcal{S}}_{\mathrm{pt}}}\left(\operatorname{colim}_{n} X_{n}, \lim _{n} Y_{n}\right) \longrightarrow \lim _{n} \operatorname{Hom}_{\mathrm{h} \widehat{\mathcal{S}}_{\mathrm{pt}}}\left(X_{n}, Y_{n}\right)
$$

est surjective et l'image réciproque de $\varphi$ par cette application s'identifie à l'ensemble $\lim _{n}^{1} \pi_{1}\left(\operatorname{hom}_{\mathrm{pt}}\left(X_{n}, Y_{n}\right), \varphi_{n}\right)$.

Démonstration. - On commence par choisir une tour $\left(Y_{n}^{\prime}\right)$ et un morphisme de tours $\left(Y_{n}\right) \rightarrow\left(Y_{n}^{\prime}\right)$ tels que chaque application $Y_{n} \rightarrow Y_{n}^{\prime}$ soit une équivalence faible et chaque application $Y_{n+1}^{\prime} \rightarrow Y_{n}^{\prime}$ soit une fibration. L'application $\lim _{n} Y_{n} \rightarrow \lim _{n} Y_{n}^{\prime}$ induit un isomorphisme en cohomologie modulo $p$ continue donc est un isomorphisme dans h $\widehat{\mathcal{S}}_{\mathrm{pt}}$. On applique ensuite la proposition à la tour de fibrations $\left(\operatorname{hom}_{\mathrm{pt}}\left(X_{n}, Y_{n}^{\prime}\right)\right)_{n}$.

Fibres homotopiques. - La proposition suivante est vraie dans toute catégorie de modèles fermée ([GJ, Chap. II, §8, lemma 8.10]) :

Proposition 1.4.4. - Soient $Z \rightarrow X \leftarrow Y$ et $Z^{\prime} \rightarrow X^{\prime} \leftarrow Y^{\prime}$ des diagrammes d'espaces profinis fibrants et $(Z \rightarrow X \leftarrow Y) \rightarrow\left(Z^{\prime} \rightarrow X^{\prime} \leftarrow Y^{\prime}\right)$ un morphisme de diagrammes. On suppose que les applications $Z \rightarrow Z^{\prime}, X \rightarrow X^{\prime}$ et $Y \rightarrow Y^{\prime}$ sont des équivalences faibles et que les applications $Y \rightarrow X$ et $Y^{\prime} \rightarrow X^{\prime}$ sont des fibrations. Alors l'application $Z \times_{X} Y \rightarrow Z^{\prime} \times_{X^{\prime}} Y^{\prime}$ est une équivalence faible.

Soit $X$ un espace profini pointé fibrant. On pointe le simplexe standard $\triangle[1]$ par la coface $\mathrm{d}^{1}: \triangle[0] \rightarrow \triangle[1]$ et on note $\mathrm{P} X$ l'espace profini pointé $\operatorname{hom}_{\mathrm{pt}}(\triangle[1], X)$. Comme l'application $\mathrm{d}^{1}$ est une cofibration triviale, l'application $\mathrm{P} X \rightarrow$ pt est une équivalence faible. 
L'application d'espaces pointés $\mathrm{d}^{0}: \triangle[0]_{+} \rightarrow \triangle[1]$ induit une fibration $\mathrm{P} X \rightarrow X$. Pour tout espace profini pointé fibrant $Y$ et toute application $Y \rightarrow X$ on appelle fibre homotopique de $Y \rightarrow X$ et on note $\mathrm{F}_{Y \rightarrow X}$ le produit fibré $Y \times_{X} \mathrm{P} X$ (qui est fibrant dans $\left.\widehat{\mathcal{S}}_{\text {pt }}\right)$.

Observons que si $Y$ est réduit au point, la fibre homotopique de l'application $Y \rightarrow X$ s'identifie à l'espace de lacets $\Omega X$.

Soit $Y \rightarrow X$ un morphisme entre espaces profinis pointés fibrants. La fibre homotopique de l'application $\mathrm{F}_{Y \rightarrow X} \rightarrow Y$ s'identifie à la limite du diagramme formé de la composée $\mathrm{P} Y \rightarrow Y \rightarrow X$ et de $\mathrm{P} X \rightarrow X$. En particulier l'équivalence faible $\mathrm{pt} \rightarrow \mathrm{P} Y$ induit une application du produit fibré pt $\times_{X} \mathrm{P} X=\Omega X$ dans $\mathrm{F}_{\mathrm{F}_{Y \rightarrow X} \rightarrow Y}$ qui est une équivalence faible par la proposition qui précède.

Nous rappelons enfin :

Proposition 1.4.5. - Soit $Z \rightarrow X \leftarrow Y$ un diagramme entre espaces pointés fibrants. On suppose que l'application $Y \rightarrow X$ est une fibration, alors :

(a) L'application $f: \pi_{0}\left(Z \times_{X} Y\right) \rightarrow \pi_{0} Z \times_{\pi_{0} X} \pi_{0} Y$ est une surjection entre ensembles pointés.

(b) Le groupe $\pi_{1} X$ opère sur l'ensemble $\pi_{0}\left(Z \times_{X} Y\right)$ au dessus de $\pi_{0} Z \times_{\pi_{0} X} \pi_{0} Y$.

(c) L'image réciproque par $f$ du point base de $\pi_{0} Z \times_{\pi_{0} X} \pi_{0} Y$ s'identifie au $\pi_{1} X$ ensemble $\pi_{1} X / \operatorname{Im}\left(\pi_{1} Z \times_{\pi_{1} X} \pi_{1} Y \rightarrow \pi_{1} X\right)$.

On en déduit une suite exacte en homotopie de type « Mayer-Vietoris ».

On peut remplacer espaces pointés fibrants par espaces profinis pointés fibrants dans la proposition ci-dessus. La démonstration est analogue au cas classique et est également conséquence du cas classique par le point (c) de la proposition 1.3.4.

Cofibres homotopiques. - Soit $Z \leftarrow X \rightarrow Y$ un diagramme d'espaces profinis. On suppose que l'application $X \rightarrow Y$ est une cofibration (i.e. est injective degré par degré). Le complexe de cochaînes continues $\mathrm{C}^{*}\left(X ; \mathbb{F}_{p}\right)$ est alors la somme amalgamée de $\mathrm{C}^{*}\left(Y ; \mathbb{F}_{p}\right)$ et de $\mathrm{C}^{*}\left(Z ; \mathbb{F}_{p}\right)$ au dessous de $\mathrm{C}^{*}\left(Z \cup_{X} Y ; \mathbb{F}_{p}\right)$ de sorte qu'on obtient la suite exacte longue de Mayer-Vietoris en cohomologie modulo $p$ continue :

$$
\cdots \longrightarrow \mathrm{H}^{*}\left(Z \cup_{X} Y\right) \longrightarrow \mathrm{H}^{*} Y \oplus \mathrm{H}^{*} Z \longrightarrow \mathrm{H}^{*} X \longrightarrow \mathrm{H}^{*+1}\left(Z \cup_{X} Y\right) \longrightarrow \cdots .
$$

On se sert de cette suite exacte pour montrer que les catégories de modèles fermées $\widehat{\mathcal{S}}$ et $\widehat{\mathcal{S}}_{\mathrm{pt}}$ sont simpliciales.

La proposition suivante est la version duale de la proposition 1.4.4 et est une application directe de la suite exacte de Mayer-Vietoris :

Proposition 1.4.6. - Soient $Z \leftarrow X \rightarrow Y$ et $Z^{\prime} \leftarrow X^{\prime} \rightarrow Y^{\prime}$ des diagrammes d'espaces profinis et $(Z \leftarrow X \rightarrow Y) \rightarrow\left(Z^{\prime} \leftarrow X^{\prime} \rightarrow Y^{\prime}\right)$ un morphisme de diagrammes. On suppose que les applications $Z \rightarrow Z^{\prime}, X \rightarrow X^{\prime}$ et $Y \rightarrow Y^{\prime}$ sont des équivalences faibles et que les applications $X \rightarrow Y$ et $X^{\prime} \rightarrow Y^{\prime}$ sont des cofibrations. Alors l'application $Z \cup_{X} Y \rightarrow Z^{\prime} \cup_{X^{\prime}} Y^{\prime}$ est une équivalence faible. 
On construit la cofibre homotopique d'un morphisme $X \rightarrow Y$ entre espaces profinis pointés comme la somme amalgamée $Y \cup_{X} \mathrm{C} X$, où $\mathrm{C} X$ est le cône $X \wedge \triangle[1]$ au dessous de $X \wedge \triangle[0]_{+}$via la coface $\mathrm{d}^{0}$. On la note $\mathrm{C}_{X \rightarrow Y}$.

On observe que si $Y$ est réduit au point alors $\mathrm{C}_{X \rightarrow Y}$ s'identifie à la suspension $\Sigma X$

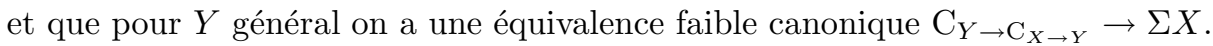

Pour terminer soit $Z \leftarrow X \rightarrow Y$ un diagramme d'espaces profinis pointés tel que l'application $X \rightarrow Y$ soit une cofibration et soit $Y^{\prime}$ un espace profini pointé fibrant. L'espace fonctionnel $\operatorname{hom}_{\mathrm{pt}}\left(Z \cup_{X} Y, Y^{\prime}\right)$ est le produit fibré

$$
\operatorname{hom}_{\mathrm{pt}}\left(Z, Y^{\prime}\right) \times_{\operatorname{hom}_{\mathrm{pt}}\left(X, Y^{\prime}\right)} \operatorname{hom}_{\mathrm{pt}}\left(Y, Y^{\prime}\right)
$$

et l'application $\operatorname{hom}_{\mathrm{pt}}\left(Y, Y^{\prime}\right) \rightarrow \operatorname{hom}_{\mathrm{pt}}\left(X, Y^{\prime}\right)$ est une fibration dans $\mathcal{S}_{\mathrm{pt}}$. On dispose alors de la proposition 1.4.5 reliant les groupes d'homotopie de ces espaces fonctionnels pour tout choix d'un point base de $\operatorname{hom}_{\mathrm{pt}}\left(Z \cup_{X} Y, Y^{\prime}\right)$.

Prenons en particulier le diagramme $Y \leftarrow X \rightarrow \mathrm{C} X$ associé à un morphisme $X \rightarrow Y$ de $\widehat{\mathcal{S}}_{\mathrm{pt}}$ et pointons les espaces fonctionnels par l'application trivial. On obtient la suite exacte de Puppe

$$
\begin{aligned}
& \operatorname{Hom}_{\mathrm{h} \widehat{\mathcal{S}}_{\mathrm{pt}}}\left(X, Y^{\prime}\right) \longleftarrow \operatorname{Hom}_{\mathrm{h} \widehat{\mathcal{S}}_{\mathrm{pt}}}\left(Y, Y^{\prime}\right) \longleftarrow \operatorname{Hom}_{\mathrm{h} \widehat{\mathcal{S}}_{\mathrm{pt}}}\left(\mathrm{C}_{X \rightarrow Y}, Y^{\prime}\right) \longleftarrow \\
& \operatorname{Hom}_{\mathrm{h} \widehat{\mathcal{S}}_{\mathrm{pt}}}\left(\Sigma X, Y^{\prime}\right) \longleftarrow \operatorname{Hom}_{\mathrm{h} \widehat{\mathcal{S}}_{\mathrm{pt}}}\left(\Sigma Y, Y^{\prime}\right) \longleftarrow \cdots .
\end{aligned}
$$

1.5. Action d'un pro- $p$-groupe abélien simplicial. — Soit $G$ un pro- $p$-groupe simplicial ; la construction classique du classifiant de $G$ fournit une fibration principale $\mathrm{E} G \rightarrow \mathrm{B} G$ de groupe $G$. Les espaces profinis $\mathrm{E} G$ et $\mathrm{B} G$ sont fibrants et l'application $\mathrm{E} G \rightarrow$ pt est une équivalence faible (voir $[\mathbf{M o 2}, 1.5]$ ).

Soit $X$ un espace profini muni d'une action de $G$ ( $X$ est muni d'une application $G \times X \rightarrow X$ dans $\widehat{\mathcal{S}}$ vérifiant les axiomes d'une action). On définit l'espace profini $X_{\mathrm{h} G}$ comme la construction de Borel $\mathrm{E} G \times{ }_{G} X$ (le quotient de $\mathrm{E} G \times X$ par l'action diagonale de $G$ ). On dispose d'une équivalence faible $G$-équivariante du produit fibré $\mathrm{E} G \times{ }_{\mathrm{B} G} X_{\mathrm{h} G}=\mathrm{E} G \times X$ (avec action principale de $G$ ) dans $X$.

Inversement soient $Y$ un espace profini fibrant et $Y \rightarrow \mathrm{B} G$ un morphisme dans $\widehat{\mathcal{S}}$. Alors le produit fibré $\mathrm{E} G \times{ }_{\mathrm{B} G} Y$ est fibrant; il est canoniquement isomorphe dans $\mathrm{h} \widehat{\mathcal{S}}$ à la fibre homotopique de $Y \rightarrow \mathrm{B} G$; il est muni d'une action principale de $G$ et l'application induite $\mathrm{E} G \times_{G}\left(\mathrm{E} G \times_{\mathrm{B} G} Y\right) \rightarrow Y$ est une équivalence faible entre espaces profinis au dessus de $\mathrm{B} G$. On dira par abus que la fibre homotopique de $Y \rightarrow \mathrm{B} G$ est munie d'une action de $G$.

Supposons maintenant que $G$ est fini simplicial, l'espace E $G$ a alors la même propriété. On définit l'espace des points fixes homotopiques $X^{\mathrm{h} G}$ comme l'espace des points fixes de l'espace fonctionnel hom(EG,X) pour l'action de $G$ à la source et au but (l'espace des applications $G$-équivariantes de $\mathrm{E} G$ dans $X$ ). Il est naturellement profini. L'espace profini des points fixes homotopiques $X^{\mathrm{h} G}$ s'interprète comme la 
fibre en l'identité de la fibration $\operatorname{hom}\left(\mathrm{B} G, X_{\mathrm{h} G}\right) \rightarrow \operatorname{hom}(\mathrm{B} G, \mathrm{~B} G)$ dans $\widehat{\mathcal{S}} ; c f$. [La1, 4.2].

Suite spectrale de Serre associée à une action principale de G. - La construction par Dress ([Dr] $)$ de la suite spectrale de Serre s'adapte au cadre profini : Soit $Y \rightarrow X$ une application entre espaces profinis fibrants. On note $E_{s, t}$ l'ensemble des diagrammes commutatifs

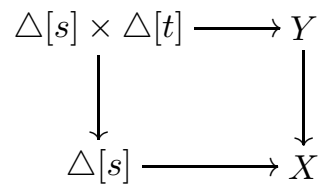

dans $\widehat{\mathcal{S}}$, où $\triangle[s]$ désigne le simplexe standard de dimension $s$ et où $\triangle[s] \times \triangle[t] \rightarrow \triangle[s]$ est la projection sur le premier facteur. La structure cosimplicial sur l'ensemble des $\triangle[s]$ induit une structure d'ensemble profini bisimplicial sur $\left(E_{s, t}\right)_{s, t}$ qu'on note $E_{\bullet},$. .

Soit $\pi$ un $p$-groupe abélien fini et notons $C^{*, *}$ le bicomplexe des cochaînes continues

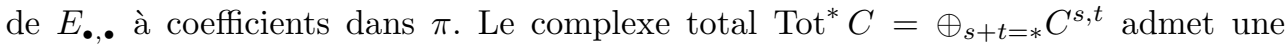
filtration décroissante par les sous-complexes $F_{s} \operatorname{Tot} C=\oplus_{s^{\prime}+t=*, s^{\prime} \geqslant s} C^{s^{\prime}, t}$.

On appelle suite spectrale de Serre en cohomologie continue à coefficients dans $\pi$ associée à l'application $Y \rightarrow X$ la suite spectrale associée à cette filtration. C'est une suite spectrale du premier cadrant avec une différentielle $\mathrm{d}_{r}$ de bidegré $(r, 1-r)$, $r \geqslant 1$, de terme $\mathrm{E}_{2}^{s, t}=\mathrm{H}^{s}\left(\left[s^{\prime}\right] \mapsto \mathrm{H}^{t} E_{s^{\prime}}, \bullet\right)$. Elle converge fortement vers l'homologie du complexe Tot $C$.

Le complexe total admet une autre filtration décroissante par les complexes

$$
F_{t}^{\prime} \operatorname{Tot} C=\oplus_{s+t^{\prime}=*, t^{\prime} \geqslant t} C^{s, t^{\prime}},
$$

d'où une seconde suite spectrale convergeant fortement vers l'homologie du même complexe total et de terme $\mathrm{E}_{2}^{\prime t, s}=\mathrm{H}^{t}\left(\left[t^{\prime}\right] \mapsto \mathrm{H}^{s} E_{\bullet}, t^{\prime}\right)$. Les applications $\triangle[t] \rightarrow \mathrm{pt}$ induisent des équivalences faibles $Y \rightarrow E_{\bullet}, t$ donc, pour tout entier positif $s$, un isomorphisme de l'objet simplicial constant $[t] \mapsto \mathrm{H} \pi^{s} Y$ dans l'objet simplicial $[t] \mapsto \mathrm{H}^{s} E_{\bullet}, t$. On en déduit que le terme $\mathrm{E}_{2}^{t, s}$ de la suite spectrale associée à la deuxième filtration est isomorphe à $\mathrm{H} \pi^{s} Y$ si $t=0$ et 0 sinon, d'où un isomorphisme canonique entre l'homologie de Tot $C$ et la cohomologie continue de $Y$ à coefficients dans $\pi$.

Rappelons que si $G$ est un pro-p-groupe simplicial et $M$ un $\pi_{0} G$-module discret (i.e. tel que les orbites des éléments de $M$ sous l'action de $\pi_{0} G$ sont finies) on définie la cohomologie continue de $G$ à coefficients dans $M$ comme la colimite, $U$ décrivant les sous-groupes distingués ouverts de $G$, des cohomologies des classifiants de $G / U$ à coefficients dans le système de coefficients locaux $M^{\pi_{0} U}([\mathbf{Q u 2}])$.

Proposition 1.5.1. - Soient $G$ un pro-p-groupe simplicial, $X$ un espace profini fibrant et $X \rightarrow \mathrm{B} G$ une application, alors le terme $\mathrm{E}_{2}^{s, t}$ de la suite spectrale de Serre associée s'identifie au $t$-ième groupe de cohomologie continue de $G$ à coefficients dans le $\pi_{0} G$-module discret $\mathrm{H} \pi^{t}\left(\mathrm{E} G \times_{\mathrm{B} G} X\right)$. 
Démonstration. - La proposition est vraie lorsque $X$ et $G$ sont finis en chaque degré. Dans le cas général, l'application $X \rightarrow \mathrm{B} G$ s'écrit comme la composée $X \rightarrow \mathrm{R} X \rightarrow \mathrm{B} G$ (car $X$ est rétract de $\mathrm{R} X$ ) pour une application $\mathrm{R} X \rightarrow \mathrm{B} G$ unique à homotopie près. L'application $\mathrm{R} X \rightarrow \mathrm{B} G$ est limite d'applications $\widehat{X}\left(i_{\alpha}\right) \rightarrow \mathrm{B}\left(G / U_{\alpha}\right)$, $i_{\alpha}$ et $U_{\alpha}$ décrivant respectivement une sous-catégorie cofinale de la catégorie index de $\widehat{X}(-)$ et un sous-ensemble final de l'ensemble des sous-groupes distingués ouverts de $G$ ordonné par l'inclusion. La suite spectrale de Serre associée à l'application $\mathrm{R} X \rightarrow \mathrm{B} G$ est alors la colimite filtrante des suites spectrales de Serre associées aux applications $\widehat{X}\left(i_{\alpha}\right) \rightarrow \mathrm{B}\left(G / U_{\alpha}\right)$. On conclut en observant que la cohomologie continue de $G$ à coefficients dans le $\pi_{0} G$-module discret $\mathrm{H} \pi^{t}\left(\mathrm{E} G \times_{\mathrm{B} G} \mathrm{R} X\right)$ est colimite des cohomologies des groupes $G / U_{\alpha}$ à coefficients dans les $\pi_{0}\left(G / U_{\alpha}\right)$-modules $\mathrm{H} \pi^{t}\left(\mathrm{E}\left(G / U_{\alpha}\right) \times_{\mathrm{B}\left(G / U_{\alpha}\right)} \widehat{X}\left(i_{\alpha}\right)\right)$ par le lemme 2.4 de $[\mathbf{Q u 2}]$.

Soient $X, Y$ deux espaces profinis munis d'une action de $G$ et $X \rightarrow Y$ une équivalence faible G-équivariante. La convergence de la suite spectrale de Serre et la description du terme $\mathrm{E}_{2}$ montrent que le morphisme induit $X_{\mathrm{h} G} \rightarrow Y_{\mathrm{h} G}$ est une équivalence faible. Supposons $X$ et $Y$ fibrants dans $\widehat{\mathcal{S}}$; les constructions de Borel associées le sont alors également. Supposons $G$ fini simplicial; on déduit de ce qui précède et de l'interprétation de l'espace profini des points fixes homotopiques que le morphisme $X^{\mathrm{h} G} \rightarrow Y^{\mathrm{h} G}$ est une équivalence faible.

1.6. Cohomologie continue des espaces profinis. - Soit $M$ le $\Omega$-spectre d'une théorie cohomologique multiplicative sur $\mathcal{S}$ ( $c f$. [Ad2, Part. III]). On dispose donc d'une suite d'espaces pointés fibrants $M_{n}, n \in \mathbb{Z}$, d'équivalences d'homotopie $M_{n} \rightarrow$ $\Omega M_{n+1}$ et d'applications $M_{n} \times M_{m} \rightarrow M_{n+m}$, etc. faisant de la suite des $M_{n}$ un objet en anneau gradué commutatif unitaire de $\mathrm{h} \mathcal{S}$.

On suppose que le spectre $M$ est -1-connexe, i.e. que les espaces $M_{n}$ sont $(n-1)$ connexes pour tout $n \geqslant 0$, et que la cohomologie modulo $p$ des espaces $M_{n}$ est de dimension finie en chaque degré pour tout $n \geqslant 1$.

\section{LEMME 1.6.1}

- Soit X un espace simplement connexe dont la cohomologie modulo $p$ est finie en chaque degré, alors l'application $(\Omega X)^{\widehat{p}} \rightarrow \Omega \widehat{X}^{p}$ est une équivalence faible (dans $\widehat{\mathcal{S}}$ ).

- Soient $X$ et $Y$ deux espaces tels que la cohomologie modulo $p$ de $X$ ou de $Y$ est finie en chaque degré, alors l'application $(X \times Y)^{\widehat{p}} \rightarrow \widehat{X}^{p} \times \widehat{Y}^{p}$ est une équivalence faible.

Démonstration. - Pour le premier point on montre que le morphisme du pro-espace constant $\Omega X$ dans $\Omega \widehat{X}(-)$ induit un isomorphisme en cohomologie modulo $p$ continue en comparant la suite spectrale d'Eilenberg-Moore en cohomologie modulo $p$ de la fibration $\Omega X \rightarrow \mathrm{PX} \rightarrow X$ (qui converge fortement parce que le groupe $\pi_{1} X$ est trivial) avec la colimite des suites spectrales d'Eilenberg-Moore associées aux fibrations 
d'espaces $p$-finis $\Omega \widehat{X}(i) \rightarrow \mathrm{P} \widehat{X}(i) \rightarrow \widehat{X}(i)$. Le second point s'obtient en comparant les formules de Künneth pour $X \times Y$ et pour $\widehat{X}(-) \times \widehat{Y}(-)$. Voir aussi [BK, chap. VI, prop. 6.5].

Le lemme indique que pour tout entier $n \geqslant 1$ l'application $\left(M_{n}\right)^{\widehat{p}} \rightarrow \Omega\left(M_{n+1}\right)^{\widehat{p}}$, composée des applications $\left(M_{n}\right)^{\widehat{p}} \rightarrow\left(\Omega M_{n+1}\right)^{\widehat{p}}$ et $\left(\Omega M_{n+1}\right)^{\widehat{p}} \rightarrow \Omega\left(M_{n+1}\right)^{\widehat{p}}$, est une équivalence faible dans $\widehat{\mathcal{S}}$. Ce n'est plus vrai pour $n=0$ si $M_{0}$ a une infinité de composantes connexes : la cohomologie modulo $p$ continue du second est de cardinal dénombrable alors que celle du premier est profinie.

On définit pour tout $n \geqslant 1$ l'espace profini pointé $\widehat{M}_{n}$ comme le $p$-complété profini $\left(M_{n}\right)^{\widehat{p}}$. Pour $n \leqslant 0$, on définit inductivement $\widehat{M}_{n}$ comme l'espace profini de lacets $\Omega\left(M_{n+1}\right)^{\widehat{p}}$. La structure d'objet en anneau commutatif gradué de la suite $\left(M_{n}\right)$ dans hS induit une structure d'objet en anneau commutatif gradué sur la suite $\left(\left(M_{n}\right)^{\widehat{p}}\right)$ dans h $\widehat{\mathcal{S}}$.

DÉfinition. - On appelle $M$-cohomologie continue d'un espace profini $X$ et on note $\widehat{M}^{*} X$ l'anneau gradué $\operatorname{Hom}_{\mathrm{h} \widehat{\mathcal{S}}}\left(X, \widehat{M}_{*}\right)$.

On note $\widehat{M}^{*}$ la $M$-cohomologie continue du point. Pour tout espace profini $X$, l'unique application $X \rightarrow$ pt fait de $\widehat{M}^{*} X$ une $\widehat{M}^{*}$-algèbre commutative unitaire. Si $X$ est pointé, on note $\widetilde{\widehat{M}}^{*} X$ le noyau du morphisme de $\widehat{M}^{*}$-modules $\widehat{M}^{*} X \rightarrow \widehat{M}^{*}$ induit par l'application du point dans $X$ (la $M$-cohomologie continue réduite de $X$ ). Le $\widehat{M}^{*}$-module $\widehat{M}^{*} X$ est alors canoniquement la somme directe de $\widetilde{M^{*}} X$ et de $\widehat{M}^{*}$.

L'objet en anneau $\widehat{M}_{*}$ de h $\widehat{\mathcal{S}}$ est en fait un objet en anneau (non unitaire) de h $\widehat{\mathcal{S}}_{\mathrm{pt}}$. La cofibration $\mathrm{pt}_{+} \rightarrow X_{+} \rightarrow X$ induit une suite exacte courte en $M$-cohomologie continue qui identifie l'idéal $\widetilde{\widehat{M}}^{*} X$ avec l'objet $\operatorname{Hom}_{\mathrm{h}} \widehat{\mathcal{S}}_{\mathrm{pt}}\left(X, \widehat{M}_{*}\right)$. Les équivalences faibles $\widehat{M}_{s} \rightarrow \Omega \widehat{M}_{s+1}$ induisent un isomorphisme $\widetilde{\widehat{M}}^{*} X \simeq \widetilde{\widehat{M}}^{*+1} \Sigma X$.

Le foncteur oubli $\widehat{\mathcal{S}}_{\mathrm{pt}} \rightarrow \mathcal{S}_{\mathrm{pt}}$ commute aux limites, au foncteur espace de lacets et aux équivalences faibles entre objets fibrants (car ces dernières sont des équivalences d'homotopie simpliciale). L'image $\left(\left|\widehat{M}_{n}\right|\right)_{n}$ du $\Omega$-spectre en anneau $\left(\widehat{M}_{n}\right)$ par le foncteur oubli $\widehat{\mathcal{S}}_{\mathrm{pt}} \rightarrow \mathcal{S}_{\mathrm{pt}}$ est un $\Omega$-spectre en anneau définissant une théorie cohomologique multiplicative sur $\mathcal{S}$ qu'on note encore $X \mapsto \widehat{M}^{*} X$ et qu'on appelle la $M$-cohomologie complétée en $p$. Les bijections naturelles de la section 1.3 donnent pour tout ensemble simplicial $W$ un isomorphisme canonique entre la $M$-cohomologie complétée en $p$ de $W$ et la $M$-cohomologie continue du complété profini $\widehat{W}^{f}$. Le morphisme de spectres en anneau $M \rightarrow \widehat{M}$ induit au niveau des coefficients un isomorphisme $\mathbb{Z}_{p} \otimes_{\mathbb{Z}} M^{*} \rightarrow \widehat{M}^{*}$ (cf. [BK, Chap. VI, §5]).

\section{EXEMPLES}

- Soient $\pi$ un groupe abélien de type fini et $\mathrm{H} \pi$ le $\Omega$-spectre formé des espaces d'Eilenberg-Mac Lane $\mathrm{K}(\pi, n)$. Alors la $\mathrm{H} \pi$-cohomologie continue d'un espace 
profini $X$ coïncide avec la cohomologie continue de $X$ à coefficients dans le groupe abélien profini $\mathbb{Z}_{p} \otimes_{\mathbb{Z}} \pi$.

- Le $\Omega$-spectre MU représentant le cobordisme complexe satisfait aux hypothèses faites sur le spectre $M$. Nous nous spécialiserons à cette théorie cohomologique dans la prochaine section.

- On peut définir la K-théorie continue d'un espace profini bien que le $\Omega$-spectre représentant la K-théorie ne soit pas connexe (il est périodique) : Notons U la colimite des groupes unitaires $\mathrm{U}(n)$ et $\mathrm{BU}$ la colimite des classifiants $\mathrm{BU}(n)$; la donnée du $p$ complété $\widehat{\mathrm{U}}^{p}$, de son espace de lacets (celui-ci s'identifie à l'espace profini $\mathbb{Z}_{p} \times \widehat{\mathrm{BU}}^{p}$ ) et de l'équivalence d'homotopie $\widehat{\mathrm{U}}^{p} \rightarrow \Omega \Omega \widehat{\mathrm{U}}^{p}$ définit un $\Omega$-spectre périodique de h $\widehat{\mathcal{S}}$ dont le $\Omega$-spectre de hS sous-jacent représente la K-théorie complétée en $p$.

\section{REMARQUES}

- On peut remplacer les hypothèses de finitude portant sur les $M_{n}$ par la condition « $M_{n}$ est connexe et de cohomologie modulo $p$ finie en chaque degré pour $n$ assez grand »: il suffit de définir $\widehat{M}_{n}$ comme l'espace de lacets itéré $\Omega^{k}\left(M_{n+k}\right)^{\widehat{p}}$ avec $k$ suffisamment grand.

- La $M$-cohomologie continue est à valeur dans la catégorie des groupes abéliens gradués. On peut concevoir des raffinements à valeurs dans d'autres catégories abéliennes, par exemple le foncteur associant à un espace profini fibrant $X$ la tour des $M$-cohomologies continues des squelettes de $X$ et à un morphisme dans h $\widehat{\mathcal{S}}$ le promorphisme induit entre les tours (on vérifie que ce foncteur définit une théorie cohomologique en observant que la tour des squelettes de la cofibre d'une application est homotopiquement pro-isomorphe à la tour des cofibres des applications induites entre les squelettes) ou encore le foncteur associant à un espace profini $X$ le pro-objet en anneau formé des ensembles $\operatorname{Hom}_{\mathrm{h} \widehat{\mathcal{S}}}(X, \widehat{M}(i))$.

Propriétés d'exactitude de la $M$-cohomologie continue. — Soient $X$ un espace profini pointé et $n$ un entier. La proposition ci-dessous (suite exacte de Milnor) est conséquence de la proposition 1.4.2 appliquée à la tour de fibrations $\left(\operatorname{hom}_{\mathrm{pt}}\left(\operatorname{Sk}_{s} X, \widehat{M}_{n}\right)\right)_{s}$ :

Proposition 1.6.2. — On a une suite exacte naturelle de groupes abéliens

$$
0 \longrightarrow \lim _{s}^{1} \widetilde{\widehat{M}}^{n-1} \mathrm{Sk}_{s} X \longrightarrow \widetilde{\widehat{M}}^{n} X \longrightarrow \lim _{s} \widetilde{\widehat{M}}^{n} \mathrm{Sk}_{s} X \longrightarrow 0 \text {. }
$$

Soient maintenant $Z \leftarrow X \rightarrow Y$ un diagramme d'espaces profinis pointés. On suppose que l'application $X \rightarrow Y$ est une cofibration dans $\widehat{\mathcal{S}}$ alors l'application induite $\operatorname{hom}_{\mathrm{pt}}\left(Y, \widehat{M}_{n}\right) \rightarrow \operatorname{hom}_{\mathrm{pt}}\left(X, \widehat{M}_{n}\right)$ est une fibration de $\mathcal{S}$ et l'espace $\operatorname{hom}_{\mathrm{pt}}\left(Z \cup_{X} Y, \widehat{M}_{n}\right)$ est le produit fibré du diagramme

$$
\operatorname{hom}_{\mathrm{pt}}\left(Z, \widehat{M}_{n}\right) \longrightarrow \operatorname{hom}_{\mathrm{pt}}\left(X, \widehat{M}_{n}\right) \longleftarrow \operatorname{hom}_{\mathrm{pt}}\left(Y, \widehat{M}_{n}\right)
$$

(voir la discussion sur les cofibres homotopiques en section 1.4). La proposition 1.4.5 entraîne la proposition suivante (suite exacte de Mayer-Vietoris) : 
Proposition 1.6.3. - Sous l'hypothèse ci-dessus, on a une suite exacte longue naturelle de groupes abéliens gradués

$$
\cdots \longrightarrow \widetilde{\widehat{M}}^{*}\left(Z \cup_{X} Y\right) \longrightarrow \widetilde{\widehat{M}}^{*} Y \oplus \widetilde{\widehat{M}}^{*} Z \longrightarrow \widetilde{\widehat{M}}^{*} X \longrightarrow \widetilde{\widehat{M}}^{*+1}\left(Z \cup_{X} Y\right) \longrightarrow \cdots
$$

\section{Structure additive de la MU-cohomologie continue des espaces profinis}

Nous étudions dans cette section la structure additive de la MU-cohomologie continue d'un espace profini, qui enrichie sa structure d'ensemble gradué et affine sa structure de $\widehat{\mathrm{MU}}^{*}$-module. Notre objectif est d'obtenir une formule de Künneth pour la MU-cohomologie continue d'un produit et une formule de coefficients universels donnant le lien entre MU-cohomologie continue et cohomologie modulo $p$ continue. Nous nous restreignons d'abord en section 2.1 aux espaces profinis sans $p$-torsion, c'est-àdire ceux dont la cohomologie continue à coefficients dans les entiers $p$-adiques est sans torsion. L'exemple fondamental est l'espace profini de lacets infini $\widehat{\mathrm{MU}}_{n}, n \in \mathbb{Z}$, par un résultat de Wilson (proposition 2.1.6). Nous traitons le cas général en section 2.2. Les arguments de passage à la limite sont importants car c'est d'abord pour les espaces profinis dont les simplexes non dégénérés sont concentrés en un nombre fini de degrés (espaces profinis de dimension finie) que nous obtenons nos résultats. Ils sont délicats et conduisent à des résultats moins forts. Nous les reprenons dans la section 4 traitant des résolutions.

Nous commençons en section 2.1 par définir une filtration naturelle décroissante $\left(\mathrm{f}^{n} \mathrm{MU}^{*}\right)_{n}$ de l'anneau $\mathrm{MU}^{*}$ telle que

- $\mathrm{MU}^{*} / \mathrm{f}^{1}$ s'identifie à l'anneau de coefficients de la cohomologie modulo $p$;

- $\mathrm{f}^{n} \mathrm{MU}^{*} / \mathrm{f}^{n+1} \mathrm{MU}^{*}$ est un $\mathbb{F}_{p}$-espace vectoriel gradué de dimension finie;

- Le complété de $\mathrm{MU}^{*}$ pour cette filtration est l'anneau de coefficients $\widehat{\mathrm{MU}}^{*}$ de la MU-cohomologie continue.

La filtration de $\mathrm{MU}^{*}$ induit une filtration par les coefficients des MU*-modules. Nous notons $\mathcal{M}$ la catégorie des $\mathrm{MU}^{*}$-modules, $\mathcal{M}_{\mathrm{f}}$ la catégorie des $\mathrm{MU}^{*}$-modules filtrés dont la filtration est plus grossière que la filtration par les coefficients, $\mathcal{M}_{\mathrm{fc}}$ la sous-catégorie pleine de $\mathcal{M}_{\mathrm{f}}$ formée des objets complets pour la filtration et $\widehat{\mathcal{L}}$ la sous-catégorie pleine de $\mathcal{M}_{\mathrm{fc}}$ formée des objets libres sur un ensemble gradué.

Nous montrons en utilisant la suite spectrale d'Atiyah-Hirzebruch qu'un espace profini de dimension finie $X$ est sans $p$-torsion si et seulement si sa MU-cohomologie continue munie de la filtration par les coefficients est dans $\widehat{\mathcal{L}}$, auquel cas l'application $\left(\widehat{\mathrm{MU}}^{*} X\right) / \mathrm{f}^{1} \rightarrow \mathrm{H}^{*} X$ est un isomorphisme (proposition 2.1.8). Dans le cas où $X$ est de dimension quelconque, la filtration par les coefficients de la MU-cohomologie continue des squelettes de $X$ induit une filtration sur $\widehat{\mathrm{MU}}^{*} X$ et l'implication dans la proposition 2.1.8 se généralise par passage à la limite.

Nous construisons pour $M$ et $N$ dans $\widehat{\mathcal{L}}$ tels que $M / \mathrm{f}^{1}$ et $N / \mathrm{f}^{1}$ sont nuls en degré assez petit, un produit tensoriel $M \widehat{\otimes} N$ dans $\widehat{\mathcal{L}}$ caractérisé par l'isomorphisme 
$(M \widehat{\otimes} N) / \mathrm{f}^{1} \simeq M / \mathrm{f}^{1} \otimes_{\mathrm{MU}^{*}} N / \mathrm{f}^{1}$, et déduisons de la suite spectrale d'Atiyah-Hirzebruch un isomorphisme $\widehat{\mathrm{MU}}^{*} X \widehat{\otimes} \widehat{\mathrm{MU}}^{*} Y \rightarrow \widehat{\mathrm{MU}}^{*}(X \times Y)$ pour $X$ et $Y$ sans $p$-torsion (proposition 2.1.13).

Nous concluons cette section en construisant pour tout ensemble gradué $S$ un espace profini d'Eilenberg-Mac Lane généralisé $\mathrm{K}(S)$ vérifiant

- $\mathrm{K}(S)$ est sans $p$-torsion;

- pour tout espace profini $X$, l'ensemble $\operatorname{Hom}_{\mathrm{h} \widehat{\mathcal{S}}}(X, \mathrm{~K}(S))$ s'identifie à l'ensemble des applications d'ensembles gradués de $S$ dans $\widehat{M U}^{*} X$.

Le foncteur $S \mapsto \widehat{\mathrm{MU}}^{*} \mathrm{~K}(S)$ est alors muni d'une structure de monade, est à valeurs dans $\widehat{\mathcal{L}}$ et la MU-cohomologie continue d'un espace profini est une algèbre sur cette monade. La MU-cohomologie continue d'un espace profini apparaît donc naturellement comme le coégalisateur d'un diagramme de $\widehat{\mathcal{L}}$.

La section 2.2 décrit la structure additive de la MU-cohomologie d'un espace profini quelconque. Nous commençons par observer qu'à la notion d'objet de $\mathcal{M}_{\mathrm{fc}}$ libre sur un ensemble gradué correspond une monade $\widehat{\mathrm{L}}$ sur la catégorie $\mathcal{E}$ ns-gr des ensembles gradués. Nous notons $\widehat{\mathcal{M}}$ la catégorie des $\widehat{\mathrm{L}}$-algèbres. $\widehat{\mathcal{M}}$ est une catégorie abélienne dont $\widehat{\mathcal{L}}$ est la sous-catégorie pleine des objets projectifs et on dispose d'un foncteur oubli exact à droite $\widehat{\mathcal{M}} \rightarrow \mathcal{M}_{\mathrm{f}}$. Deux sous-catégories pleines de $\widehat{\mathcal{M}}$ sont familières : La sous-catégorie pleine $\widehat{\mathcal{M}}^{0}$ de $\widehat{\mathcal{M}}$ formée des $\widehat{\mathrm{L}}$-algèbres nulles en degré assez grand s'identifie à la catégorie des $\mathrm{MU}^{*}$-modules Ext-p-complets et nuls en degré assez grand (proposition 2.2.1); celle formée des $\widehat{L}$-algèbres complètes pour la filtration s'identifie à une sous-catégorie pleine de $\mathcal{M}_{\mathrm{fc}}$ que nous explicitons (proposition 2.2.8). La sous-catégorie pleine $\widehat{\mathcal{M}}^{0}$ se révélera cruciale dans l'utilisation des résolutions libres (section 4.1).

Nous étudions les notions de sous- $\widehat{L}$-algèbre et $\widehat{L}$-algèbre quotient, en particulier pour définir une filtration squelettale d'une $\widehat{\mathrm{L}}$-algèbre qui mime la filtration de $\widehat{\mathrm{MU}}^{*} X$ induite par la filtration squelettale d'un espace profini $X$. (Nous utiliserons la filtration squelettale pour étendre à $\widehat{\mathcal{M}}$ entier une construction ou un résultat sur les objets de $\widehat{\mathcal{M}}^{0}$.)

Nous étendons le bifoncteur $-\widehat{\otimes}-$ en un produit tensoriel sur $\widehat{\mathcal{M}}$ additif et exact à droite en chacune des variables, et étudions la commutation du foncteur oubli $\widehat{\mathcal{M}} \rightarrow \mathcal{M}$ au produit tensoriel sous des hypothèses de finitude (proposition 2.2.11). Nous avons en particulier des isomorphismes

- $M \widehat{\otimes}\left(\mathrm{MU}^{*} / \mathrm{f}^{n}\right) \simeq M / \mathrm{f}^{n}$ pour tout $M$ dans $\widehat{\mathcal{M}}$,

- $(M \widehat{\otimes} N) / \mathrm{f}^{n} \simeq M / \mathrm{f}^{n} \otimes_{\mathrm{MU}^{*}} N / \mathrm{f}^{n}$ pour tout $M, N$ dans $\widehat{\mathcal{M}}^{0}$.

Nous revenons pour conclure sur la présentation de la MU-cohomologie continue des espaces profinis : L'unité d'adjonction $X \rightarrow \mathrm{K}\left(\widehat{\mathrm{MU}}^{*} X\right)$ induit un morphisme $\widehat{\mathrm{MU}}^{*} \mathrm{~K}\left(\widehat{\mathrm{MU}}^{*} X\right) \rightarrow \widehat{\mathrm{MU}}^{*} X$ faisant de $\widehat{\mathrm{MU}}^{*} X$ une $\widehat{\mathrm{L}}$-algèbre quotient de la $\widehat{\mathrm{L}}$-algèbre 
libre $\widehat{\mathrm{MU}}^{*} \mathrm{~K}\left(\widehat{\mathrm{MU}}^{*} X\right)$. (Lorsque $X$ est sans $p$-torsion, cette structure de $\widehat{\mathrm{L}}$-algèbre coïncide avec la structure d'objet de $\widehat{\mathcal{L}}$ de $\widehat{M U}^{*} X$ décrite en section 2.1.) On déduit également de cette présentation un morphisme $\widehat{\mathrm{MU}}^{*} X \widehat{\otimes} \widehat{\mathrm{MU}}^{*} Y \rightarrow \widehat{\mathrm{MU}}^{*}(X \times Y)$ et sa version pointée $\widetilde{\widehat{\mathrm{MU}}}^{*} X \widehat{\otimes} \widehat{\widehat{\mathrm{MU}}}{ }^{*} Y \rightarrow \widetilde{\widehat{\mathrm{MU}}^{*}}(X \wedge Y)$, que nous étudierons en section 4.2.

\section{1. $\mathrm{MU}^{*}$-modules filtrés libres et espaces profinis sans $p$-torsion}

$\mathrm{MU}^{*}$-modules filtrés libres. - On note $\mathcal{M}$ la catégorie des $\mathrm{MU}^{*}$-modules.

Rappelons que l'anneau de coefficients $\mathrm{MU}^{*}$ est une $\mathbb{Z}$-algèbre polynomiale en des générateurs $x_{n}$ de degré $-2 n, n$ décrivant $\mathbb{N}-\{0\}$ (cf. [Ad2 2, part. II] $)$.

Soit $n$ un entier positif; on note $\mathrm{f}^{n} \mathrm{MU}^{*}$ l'idéal $p^{n} \mathrm{MU}^{* \leqslant 0}+\cdots+p^{0} \mathrm{MU}^{* \leqslant-n}$ de $\mathrm{MU}^{*}$, où $\mathrm{MU}^{* \leqslant n}$ désigne l'idéal formé des éléments de degré inférieur ou égal à $n$. L'ensemble des $\mathrm{f}^{n} \mathrm{MU}^{*}$ forme une filtration décroissante de $\mathrm{MU}^{*}$. L'idéal $\mathrm{f}^{1} \mathrm{MU}^{*}$ est le noyau du morphisme $\mathrm{MU}^{*} \rightarrow(\mathrm{HZ} / p)^{*}=\mathbb{Z} / p$ induit par l'orientation $\mathrm{MU} \rightarrow$ $\mathrm{HZ} / p$ et les quotients $\mathrm{f}^{n} \mathrm{MU}^{*} / \mathrm{f}^{n+1} \mathrm{MU}^{*}$ sont des $\mathbb{Z} / p$-espaces vectoriels gradués de dimension totale finie. Le complété de $\mathrm{MU}^{*}$ pour cette filtration s'identifie à l'anneau de coefficients de la MU-cohomologie complétée en $p$.

Soit $M$ un $\mathrm{MU}^{*}$-module; la filtration de $\mathrm{MU}^{*}$ induit une filtration naturelle sur $M$ :

$$
M=\mathrm{f}^{0} \mathrm{MU}^{*} \cdot M \supset \mathrm{f}^{1} \mathrm{MU}^{*} \cdot M \supset \cdots
$$

qu'on appelle filtration par les coefficients. On dispose d'un morphisme canonique de $M$ dans son complété $\widehat{M}=\lim _{n} M /\left(\mathrm{f}^{n} \mathrm{MU}^{*} \cdot M\right)$. On observera que $\widehat{M}$ n'est pas nécessairement complet pour la filtration par les coefficients; par exemple si $M$ est un MU*-module libre, $\widehat{M}$ est complet si et seulement si $M$ est nul en degré assez grand. Cependant $\widehat{M}$ possède une filtration plus grossière que la filtration par les coefficients définie par $\mathrm{f}^{n} \widehat{M}=\operatorname{Ker}\left(\widehat{M} \rightarrow M /\left(\mathrm{f}^{n} \mathrm{MU}^{*} \cdot M\right)\right)$, qu'on appelle filtration limite de $\widehat{M}$ et pour laquelle $\widehat{M}$ est complet (cette filtration coïncide avec la filtration par les coefficients lorsque $M$ est nul en degré assez grand). Observons que la structure de $\mathrm{MU}^{*}$-module sur $M$ induit une structure naturelle de $\widehat{\mathrm{MU}}^{*}$-module sur le complété $\widehat{M}$.

On note $\mathcal{M}_{\mathrm{f}}$ la catégorie formée des $\mathrm{MU}^{*}$-modules $M$ munis d'une filtration décroissante

$$
M=\mathrm{f}^{0} M \supset \mathrm{f}^{1} M \supset \cdots
$$

plus grossière que la filtration par les coefficients (donc telle que les quotients $M / \mathrm{f}^{n} M$ sont des $\mathrm{MU}^{*} / \mathrm{f}^{n} \mathrm{MU}^{*}$-modules) et des morphismes de $\mathrm{MU}^{*}$-modules respectant la filtration, et $\mathcal{M}_{\mathrm{fc}}$ la sous-catégorie pleine de $\mathcal{M}_{\mathrm{f}}$ formée des objets complets pour la filtration (c'est à dire des objets $M \in \mathcal{M}_{\mathrm{f}}$ tels que l'application $M \rightarrow \lim _{n} M / \mathrm{f}^{n} M$ est un isomorphisme). Ce sont des catégories additives.

Pour faire court, on notera $M / \mathrm{f}^{n}$ le quotient $M / \mathrm{f}^{n} M$ d'un objet $M$ de $\mathcal{M}_{\mathrm{f}}$.

La limite dans $\mathcal{M}_{\mathrm{f}}$ d'un diagramme d'objets $\left(M_{\alpha}\right)$ est le $\mathrm{MU}^{*}$-module $M_{\infty}$ limite des $\mathrm{MU}^{*}$-modules $M_{\alpha}$ muni de la filtration définie par $\mathrm{f}^{n} M_{\infty}=\operatorname{Ker}\left(M_{\infty} \rightarrow\right.$ $\left.\lim _{\alpha}\left(M_{\alpha} / \mathrm{f}^{n}\right)\right)$. Les foncteurs oubli $\mathcal{M}_{\mathrm{fc}} \rightarrow \mathcal{M}_{\mathrm{f}}$ et $\mathcal{M}_{\mathrm{f}} \rightarrow \mathcal{M}$ commutent donc aux 
limites. Pour tout entier $n$ le foncteur $\mathcal{M}_{\mathrm{fc}} \rightarrow \mathcal{M}, M \mapsto M / \mathrm{f}^{n}$ commute aux limites, mais pas son extension à $\mathcal{M}_{\mathrm{f}}$ entier.

La colimite dans $\mathcal{M}_{\mathrm{f}}$ de $\left(M_{\alpha}\right)$ est le $\mathrm{MU}^{*}$-module colimite des $M_{\alpha}$ muni de la filtration définie par : $\mathrm{f}^{n} M$ est le sous-module de $\operatorname{colim}_{\alpha} M_{\alpha}$ engendré par les images $\operatorname{des} \mathrm{f}^{n} M_{\alpha}$. Cette fois le foncteur $\mathcal{M}_{\mathrm{f}} \rightarrow \mathcal{M}, M \mapsto M / \mathrm{f}^{n}$ commute aux colimites.

Lemme 2.1.1. - Le foncteur de $\mathcal{M}$ dans $\mathcal{M}_{\mathrm{fc}}$ qui associe à un objet $M$ son complété $\widehat{M}$ muni de la filtration limite est adjoint à gauche du foncteur « oubli de la filtration».

On note enfin $\widehat{\mathcal{L}}$ la sous-catégorie pleine de $\mathcal{M}_{\mathrm{fc}}$ formée des objets isomorphes au complété d'un $\mathrm{MU}^{*}$-module libre muni de la filtration limite. L'importance de la catégorie $\widehat{\mathcal{L}}$ pour notre propos vient de ce que la MU-cohomologie continue d'un espace profini «sans p-torsion» est naturellement un objet de $\widehat{\mathcal{L}}$ (propositions 2.1.8 et 2.1.11). Les objets de $\widehat{\mathcal{L}}$ sont caractérisés par le lemme suivant :

Lemme 2.1.2. - Soit $M$ un objet de $\mathcal{M}_{\mathrm{f}}$, alors $M$ est dans $\widehat{\mathcal{L}}$ si et seulement si les conditions suivantes sont vérifiées:

(1) L'objet $M$ est complet pour sa filtration.

(2) Pour tout entier $n$, la surjection $\mathrm{MU}^{*} / \mathrm{f}^{n} \otimes_{\mathrm{MU}^{*}} M / \mathrm{f}^{n+1} \rightarrow M / \mathrm{f}^{n}$ est un isomorphisme.

(3) Pour tout entier $n$, la suite exacte $0 \rightarrow \mathrm{f}^{n} \mathrm{MU}^{*} / \mathrm{f}^{n+1} \mathrm{MU}^{*} \rightarrow \mathrm{MU}^{*} / \mathrm{f}^{n+1} \rightarrow$ $\mathrm{MU}^{*} / \mathrm{f}^{n} \rightarrow 0$ induit une suite exacte

$$
0 \longrightarrow \mathrm{f}^{n} \mathrm{MU}^{*} / \mathrm{f}^{n+1} \mathrm{MU}^{*} \otimes_{\mathrm{MU}^{*}} M / \mathrm{f}^{1} \longrightarrow M / \mathrm{f}^{n+1} \longrightarrow M / \mathrm{f}^{n} \longrightarrow 0 .
$$

Remarque. - Le terme $\mathrm{f}^{n} \mathrm{MU}^{*} / \mathrm{f}^{n+1} \mathrm{MU}^{*} \otimes_{\mathrm{MU}^{*}} M / \mathrm{f}^{1}$ dans l'énoncé de la condition (3) s'identifie au produit tensoriel de $\mathrm{f}^{n} \mathrm{MU}^{*} / \mathrm{f}^{n+1} \mathrm{MU}^{*}$ et de $M / \mathrm{f}^{1}$ au dessus de $\mathbb{Z} / p$ via le morphisme canonique $\mathrm{MU}^{*} \rightarrow \mathbb{Z} / p$.

Démonstration. - Soit $L$ un $\mathrm{MU}^{*}$-module libre. Les quotients $\widehat{L} / \mathrm{f}^{n}$ s'identifient par définition aux quotients $L /\left(\mathrm{f}^{n} \mathrm{MU}^{*} \cdot L\right)$ d'où les propriétés (1), (2) et (3).

Réciproquement soit $M$ un objet de $\mathcal{M}_{\mathrm{f}}$ vérifiant les propriétés (1), (2) et (3). Soit $S$ une base du $\mathbb{F}_{p}$-espace vectoriel gradué $M / \mathrm{f}^{1}$ et $L$ le $\mathrm{MU}^{*}$-module libre de base $S$. L'application canonique $L \rightarrow M / \mathrm{f}^{1}$ se relève en un morphisme de $\mathrm{MU}^{*}$-modules $L \rightarrow M$. La filtration de $M$ étant plus grossière que la filtration par les coefficients, le morphisme $L \rightarrow M$ induit pour tout entier $n$ un morphisme $L /\left(\mathrm{f}^{n} \mathrm{MU}^{*} \cdot L\right) \rightarrow M / \mathrm{f}^{n}$. Ce dernier est un isomorphisme pour $n=1$ donc un isomorphisme pour tout $n$ par récurrence en utilisant la condition (3). Le morphisme $\widehat{L} \rightarrow M$ obtenu par passage à la limite est donc un isomorphisme dans $\mathcal{M}_{\mathrm{f}}$.

Remarque. - Soit $M$ un $\mathrm{MU}^{*}$-module filtré nul en degré assez grand; alors $M$ est dans $\widehat{\mathcal{L}}$ si et seulement si la filtration de $M$ est la filtration par les coefficients et si le $\mathrm{MU}^{*}$-module sous-jacent à $M$ est le $\mathrm{MU}^{*}$-module obtenu par extension des scalaires 
$\mathrm{MU}^{*} \otimes_{\mathbb{Z}} N$ pour un certain groupe abélien gradué $N$ complet pour la filtration $p$ adique et sans torsion. Inversement le $M^{*}$-module sous-jacent à un objet $M$ de $\widehat{\mathcal{L}}$ s'écrit $\mathrm{MU}^{*} \otimes_{\mathbb{Z}} N$ pour un certain groupe abélien gradué $N$ si et seulement si $M$ est nul en degré assez grand.

Observons que la sous-catégorie $\widehat{\mathcal{L}}$ de $\mathcal{M}_{\mathrm{f}}$ est stable par produits quelconques (c'est une conséquence du lemme 2.1.2) en particulier par sommes finies.

Proposition 2.1.3. - Soient $M \rightarrow N$ un morphisme de $\widehat{\mathcal{L}}, K$ le noyau et $Q$ le conoyau du morphisme de $\mathrm{MU}^{*}$-modules sous-jacent, munis de la filtration induite par celle de $M$ et $N$ respectivement.

(a) Si l'application $M / \mathrm{f}^{1} \rightarrow N / \mathrm{f}^{1}$ est surjective alors $K$ est dans $\widehat{\mathcal{L}}$ et la suite $0 \rightarrow K \rightarrow M \rightarrow N \rightarrow 0$ est une suite exacte dans $\mathcal{M}$ scindée dans $\widehat{\mathcal{L}}$.

(b) Si l'application $M / \mathrm{f}^{1} \rightarrow N / \mathrm{f}^{1}$ est injective alors $Q$ est dans $\widehat{\mathcal{L}}$ et la suite $0 \rightarrow M \rightarrow N \rightarrow Q \rightarrow 0$ est une suite exacte dans $\mathcal{M}$ scindée dans $\widehat{\mathcal{L}}$.

Démonstration. - Soit $d: M \rightarrow N$ un morphisme de $\widehat{\mathcal{L}}$ induisant une surjection $M / \mathrm{f}^{1} \rightarrow N / \mathrm{f}^{1}$ et notons $K_{n}$ le noyau du morphisme $M / \mathrm{f}^{n} \rightarrow N / \mathrm{f}^{n} ; K_{n}$ est un $\mathrm{MU}^{*} / \mathrm{f}^{n}$-module. On montre par récurrence sur $n$ en utilisant la condition (3) du lemme 2.1.2 que l'application $M / \mathrm{f}^{n} \rightarrow N / \mathrm{f}^{n}$ est surjective. Le fait que $N / \mathrm{f}^{n}$ est un $\mathrm{MU}^{*} / \mathrm{f}^{n}$-module libre implique alors que l'application $\mathrm{MU}^{*} / \mathrm{f}^{n} \otimes_{\mathrm{MU}^{*}} K_{n+1} \rightarrow K_{n}$ est un isomorphisme; en particulier l'application $K_{n+1} \rightarrow K_{n}$ est surjective. Posons $K=\lim _{n} K_{n}$ qu'on filtre par $\mathrm{f}^{n} K=\operatorname{Ker}\left(K \rightarrow K_{n}\right)$; alors $K$ est dans $\mathcal{M}_{\mathrm{f}}$ et vérifie les conditions (1), (2) et (3) du lemme 2.1.2 donc est dans $\widehat{\mathcal{L}}$. De plus les suites exactes $0 \rightarrow K_{n} \rightarrow M / \mathrm{f}^{n} \rightarrow N / \mathrm{f}^{n} \rightarrow 0$ induisent en passant à la limite sur $n$ une suite exacte $0 \rightarrow K \rightarrow M \rightarrow N \rightarrow 0$. Il reste à démontrer que cette suite exacte est scindée dans $\widehat{\mathcal{L}}$. Soit $L$ un $\mathrm{MU}^{*}$-module libre et $L \rightarrow N$ un morphisme dans $\mathcal{M}$ induisant un isomorphisme $\widehat{L} \rightarrow N$ dans $\widehat{\mathcal{L}}$. Comme l'application $M \rightarrow N$ est surjective, le morphisme $L \rightarrow N$ se relève en un morphisme $L \rightarrow M$, d'où par adjonction un relèvement de l'isomorphisme $\widehat{L} \rightarrow N$ en un morphisme $\widehat{L} \rightarrow M$, c'est à dire une section du morphisme $M \rightarrow N$ dans $\widehat{\mathcal{L}}$; notons la $s$. On vérifie que le morphisme Id $-s d: M \rightarrow M$ induit un rétract $s^{\prime}$ de $K \rightarrow M$ dans $\widehat{\mathcal{L}}$ et que le morphisme $\left(s^{\prime}, d\right): M \rightarrow K \times N$ est un isomorphisme. Le fait que la filtration de $K$ soit induite par celle de $M$ en résulte, d'où le point (a).

Le point (b) est similaire : Soit $Q$ le conoyau d'un morphisme $M \rightarrow N$ de $\widehat{\mathcal{L}}$, muni de la filtration induite par celle de $N$; on vérifie de la même façon que si $M / \mathrm{f}^{1} \rightarrow N / \mathrm{f}^{1}$ est injective alors la suite $0 \rightarrow M / \mathrm{f}^{n} \rightarrow N / \mathrm{f}^{n} \rightarrow Q / \mathrm{f}^{n} \rightarrow 0$ est exacte pour tout $n$ et $Q$ est dans $\widehat{\mathcal{L}}$.

Corollaire 2.1.4. - Soit $M \rightarrow N$ un morphisme de $\widehat{\mathcal{L}}$; les conditions suivantes sont équivalentes :

(i) Le morphisme de $\mathrm{MU}^{*}$-modules sous-jacent est un isomorphisme. 
(ii) Le morphisme de $\mathbb{F}_{p}$-espaces vectoriels gradués $M / \mathrm{f}^{1} \rightarrow N / \mathrm{f}^{1}$ est un isomorphisme.

(iii) Le morphisme $M \rightarrow N$ est un isomorphisme de $\widehat{\mathcal{L}}$.

Démonstration. - La condition (iii) implique clairement les conditions (i) et (ii). Si l'une des conditions (i) ou (ii) est satisfaite alors l'application $M / \mathrm{f}^{1} \rightarrow N / \mathrm{f}^{1}$ est surjective et on obtient par la proposition 2.1.3 une suite $0 \rightarrow K \rightarrow M \rightarrow N \rightarrow 0$ exacte dans $\mathcal{M}$ et scindée dans $\widehat{\mathcal{L}}$. Si la condition (i) est satisfaite, $K$ est nul et la section de $M \rightarrow N$ est un inverse. Si la condition (ii) est satisfaite, le quotient $K / \mathrm{f}^{1}$ est nul d'où on déduit en utilisant le lemme 2.1.2 que $K$ est nul; on est ramené à la condition (i).

Espaces profinis sans p-torsion. - Rappelons que la MU-cohomologie continue d'un espace profini est une $\widehat{\mathrm{MU}}^{*}$-algèbre ( $c f$. section 1.6).

Un espace profini $X$ est dit de dimension finie s'il est égal à son squelette $n$-ième $\mathrm{Sk}_{n} X$ pour un certain entier $n$. On munit la MU-cohomologie continue des espaces profinis de dimension finie de la filtration par les coefficients. Elle n'est pas nécessairement complète.

Soit $X$ un espace profini quelconque. La filtration par les coefficients de la MUcohomologie continue des squelettes de $X$ induit une filtration de la MU-cohomologie continue de $X$ définie par $\mathrm{f}^{n} \widehat{\mathrm{MU}}^{*} X=\operatorname{Ker}\left(\widehat{\mathrm{MU}}^{*} X \rightarrow \lim _{s}\left(\left(\widehat{\mathrm{MU}}^{*} \mathrm{Sk}_{s} X\right) / \mathrm{f}^{n}\right)\right)$ qu'on appelle filtration limite de $\widehat{\mathrm{MU}}^{*} X$ et pour laquelle $\widehat{\mathrm{MU}}^{*} X$ est un objet de $\mathcal{M}_{\mathrm{f}}$. ( $\mathrm{Si}$ $\widehat{\mathrm{MU}}^{*} X$ est la limite comme $\mathrm{MU}^{*}$-module de la tour des $\widehat{\mathrm{MU}}^{*} \mathrm{Sk}_{s} X$ alors il en est la limite dans $\mathcal{M}_{\mathrm{f}}$.) Nous verrons que cette filtration est complète lorsque $X$ est le complété profini d'un espace mais ce n'est pas le cas en général. Toute équivalence faible $X \rightarrow X^{\prime}$ dans $\widehat{\mathcal{S}}$ induit un isomorphisme $\widehat{\mathrm{MU}}^{*} X^{\prime} \rightarrow \widehat{\mathrm{MU}}^{*} X$ entre $\mathrm{MU}^{*}$-modules et un pro-isomorphisme $\left(\widehat{\mathrm{MU}}^{*} \mathrm{Sk}_{s} X^{\prime}\right)_{s} \rightarrow\left(\widehat{\mathrm{MU}}^{*} \mathrm{Sk}_{s} X\right)_{s}$ entre tours de $\mathrm{MU}^{*}$-modules, donc un isomorphisme $\widehat{\mathrm{MU}}^{*} X^{\prime} \rightarrow \widehat{\mathrm{MU}}^{*} X$ dans $\mathcal{M}_{\mathrm{f}}$. Observons que la filtration de $\widehat{\mathrm{MU}}^{*} X$ est une filtration de $\widehat{\mathrm{MU}}^{*}$-algèbres.

Nous avons tout fait pour que le morphisme $\widehat{\mathrm{MU}}^{*} X \rightarrow \mathrm{H}^{*} X$, induit par le morphisme de spectres $\mathrm{MU} \rightarrow \mathrm{H} \mathbb{Z} / p$, se factorise par la projection $\widehat{\mathrm{MU}}^{*} X \rightarrow \widehat{\mathrm{MU}}^{*} X / \mathrm{f}^{1}$.

Proposition-DÉfinition 2.1.5. - Soit X un espace profini; les conditions suivantes sont équivalentes :

(i) Pour tout entier positif $v$ la réduction modulo $p: \mathbb{Z} / p^{v} \rightarrow \mathbb{Z} / p$ induit une surjection $\left(\mathrm{H} \mathbb{Z} / p^{v}\right)^{*} X \rightarrow \mathrm{H}^{*} X$.

(ii) La cohomologie continue de $X$ à coefficients dans le groupe profini des entiers p-adiques est sans torsion.

Si elles sont vérifiées, on dit que $X$ est sans p-torsion.

Démonstration. - (i) implique (ii) : la suite exacte de coefficients $0 \rightarrow \mathbb{Z} / p^{v} \rightarrow$ $\mathbb{Z} / p^{v+1} \rightarrow \mathbb{Z} / p \rightarrow 0$ induit une suite exacte courte en cohomologie continue. On en 
déduit par récurrence que les applications $\mathrm{H}^{*} X \rightarrow\left(\mathrm{HZ} / p^{v}\right)^{*} X$ sont injectives puis, en utilisant la suite exacte de coefficients $0 \rightarrow \mathbb{Z} / p \rightarrow \mathbb{Z} / p^{v+1} \rightarrow \mathbb{Z} / p^{v} \rightarrow 0$, que la tour $\left(\left(\mathrm{HZ} / p^{v}\right)^{*} X\right)_{v}$ est une tour de surjections. On en déduit que la cohomologie continue de $X$ à coefficients dans les entiers $p$-adiques, qui se surjecte sur la limite de cette tour par le corollaire 1.4.3, se surjecte sur la cohomologie modulo $p$ continue de $X$. On conclut en observant la suite exacte longue en cohomologie associée à la suite exacte de coefficients $0 \rightarrow \mathbb{Z}_{p} \rightarrow \mathbb{Z}_{p} \rightarrow \mathbb{Z} / p \rightarrow 0$.

Inversement cette suite exacte longue montre sous l'hypothèse (ii) que l'application $\mathrm{HZ}_{p}{ }^{*} X \rightarrow \mathrm{H}^{*} X$ est surjective, donc aussi les applications $\left(\mathrm{HZ} / p^{v}\right)^{*} X \rightarrow \mathrm{H}^{*} X$ puisque la première se factorise par chacune des dernières.

\section{EXEMPLES}

- Soient $X$ un espace profini et $s$ un entier positif. Le quotient $\mathrm{Sk}_{s+1} X / \mathrm{Sk}_{s} X$ est la limite dans $\widehat{\mathcal{S}}$ d'un pro-objet de la catégorie des bouquets finis de sphères de dimension $s+1$; il est sans $p$-torsion par le critère (i) de la définition.

- Le complété profini d'un espace $X$ est sans $p$-torsion si et seulement si la cohomologie de $X$ à coefficients dans les entiers $p$-adiques est sans torsion. Les résultats de Wilson $([\mathbf{W i}])$ impliquent en particulier la proposition capitale :

Proposition 2.1.6. - Pour tout entier n l'espace profini $\widehat{\mathrm{MU}}_{n}$ est sans p-torsion.

Démonstration. - On utilise la description par Wilson de l'homologie entière des espaces $\mathrm{MU}_{n}$. Le cas $n \geqslant 1$ vient de ce que $\widehat{\mathrm{MU}}_{n}$ est le $p$-complété profini d'un espace dont l'homologie entière est libre en chaque degré.

Pour $n \leqslant 0$ l'espace $\mathrm{MU}_{2 n+1}$ est le produit d'un produit de cercles $\mathrm{S}^{1}$ et d'un espace 2-connexe $X_{2 n+1}$, ceci parce $\mathrm{MU}_{2 n+1}$ est un H-espace et $\pi_{*} \mathrm{MU}_{2 n+1}$ est un $\mathbb{Z}$ module libre nul en degré pair $\left(c f\right.$. [Wi, section 3]). L'espace profini $\widehat{\mathrm{MU}}_{n}=\Omega^{1-n} \widehat{\mathrm{MU}}_{1}$ est alors le $p$-complété profini de $\mathrm{MU}_{n}$ si $n$ est impair et le produit d'un $\mathbb{F}_{p}$-espace vectoriel profini simplicial constant avec le $p$-complété profini de $\Omega X_{n+1}$ si $n$ est pair. Les espaces $X_{2 n+1}$ et $\Omega X_{2 n+1}$ sont d'homologie entière libre en chaque degré d'après [Wi] donc leurs $p$-complétés profinis sont sans $p$-torsion, donc également les espaces profinis $\widehat{\mathrm{MU}}_{n}$.

Indiquons également :

LEMME 2.1.7

(a) Un espace profini est sans p-torsion si et seulement si chacun de ses squelettes est sans p-torsion.

(b) Soit $X \rightarrow X^{0}$ une application entre espaces profinis. On suppose que $X^{0}$ est sans p-torsion et que le morphisme $\mathrm{H}^{*} X^{0} \rightarrow \mathrm{H}^{*} X$ est surjectif en chaque degré; alors $X$ est sans $p$-torsion. 
Démonstration. - Pour le point (a) soit $X$ un espace profini. La suite exacte longue en cohomologie associée à la suite $\mathrm{Sk}_{s} X \rightarrow X \rightarrow X / \mathrm{Sk}_{s} X$ montre que le groupe $\left(\mathrm{HZ}_{p}\right)^{k} \mathrm{Sk}_{s} X$ est égal à $\left(\mathrm{HZ}_{p}\right)^{k} X$ pour $k<s$ et est une extension d'un sous-groupe de $\left(\mathrm{HZ}_{p}\right)^{s+1}\left(X / \mathrm{Sk}_{s} X\right)$ par $\left(\mathrm{HZ}_{p}\right)^{s} X$ pour $k=s$. Le groupe gradué $\left(\mathrm{HZ}_{p}\right)^{*} X$ est donc sans torsion si chacun des $\left(\mathrm{HZ}_{p}\right)^{*} \mathrm{Sk}_{s} X$ l'est. Inversement si $\left(\mathrm{HZ}_{p}\right)^{*} X$ est sans torsion il suffit de montrer que le groupe $\left(\mathrm{HZ}_{p}\right)^{s+1}\left(X / \mathrm{Sk}_{s} X\right)$ est sans torsion pour conclure que $\left(\mathrm{HZ}_{p}\right)^{*} \mathrm{Sk}_{s} X$ est sans torsion. Or cela vient de ce que le groupe $\left(\mathrm{HZ}_{p}\right)^{s+1}\left(X / \mathrm{Sk}_{s} X\right)$ s'injecte dans $\left(\mathrm{H}_{p}\right)^{s+1}\left(\mathrm{Sk}_{s+1} X / \mathrm{Sk}_{s} X\right)$ et de ce que le quotient $\mathrm{Sk}_{s+1} X / \mathrm{Sk}_{s} X$ est la limite dans $\widehat{\mathcal{S}}$ d'un pro-bouquet fini de sphères donc est sans $p$-torsion.

Pour le point (b) on observe que pour tout entier positif $v$ les deux composées $\left(\mathrm{H} \mathbb{Z} / p^{v}\right)^{*} X^{0} \rightarrow\left(\mathrm{H} \mathbb{Z} / p^{v}\right)^{*} X \rightarrow \mathrm{H}^{*} X$ et $\left(\mathrm{HZ} / p^{v}\right)^{*} X^{0} \rightarrow \mathrm{H}^{*} X^{0} \rightarrow \mathrm{H}^{*} X$ sont égales donc le morphisme $\left(\mathrm{HZ} / p^{v}\right)^{*} X \rightarrow \mathrm{H}^{*} X$ est surjectif en chaque degré.

Puisque le morphisme de spectres $\mathrm{MU} \rightarrow \mathrm{H} \mathbb{Z} / p$ se factorise par $\mathrm{H} \mathbb{Z} / p^{v} \rightarrow \mathrm{H} \mathbb{Z} / p$ pour tout entier $v$, un espace profini est sans $p$-torsion dès que le morphisme $\widehat{\mathrm{MU}}^{*} X \rightarrow$ $\mathrm{H}^{*} X$ est surjectif en chaque degré. Les résultats qui suivent donnent une réciproque.

Proposition 2.1.8. - Soit $X$ un espace profini de dimension finie; alors les conditions suivantes sont équivalentes :

(i) L'espace profini $X$ est sans p-torsion.

(ii) La MU-cohomologie continue de X munie de la filtration par les coefficients est dans $\widehat{\mathcal{L}}$.

Si elles sont vérifiées, l'application canonique $\widehat{\mathrm{MU}}^{*} X / \mathrm{f}^{1} \rightarrow \mathrm{H}^{*} X$ est un isomorphisme.

Démonstration. - La proposition se démontre en introduisant une suite spectrale d'Atiyah-Hirzebruch reliant la MU-cohomologie continue d'un espace profini avec sa cohomologie continue à coefficients dans le groupe abélien profini $\widehat{\mathrm{MU}}^{*}$.

Soit $M$ le $\Omega$-spectre d'une théorie cohomologique sur $\mathcal{S}$. On suppose que pour tout entier $n \geqslant 1, M_{n}$ est $(n-1)$-connexe et de cohomologie modulo $p$ finie en chaque degré. On note alors $\widehat{M}_{n}$ le $n$-ième terme du $\Omega$-spectre représentant la $M$-cohomologie continue sur $\widehat{\mathcal{S}}$ et $\widehat{M}_{n}(-)$ le pro-espace $p$-fini pointé dont $\widehat{M}_{n}$ est la limite $\left(\widehat{M}_{n}(-)\right.$ est le pro-p-complété de $M_{n}$ si $\left.n \geqslant 1\right)$. Les hypothèses sur $M_{n}$ garantissent que $\widehat{M}_{n}(-)$ est isomorphe dans prop-h $\mathcal{S}_{\mathrm{pt}}$ à une tour d'espaces $p$-finis.

Rappelons que $\widehat{M}^{t}$ désigne le pro-p-groupe $\pi_{0} \widehat{M}_{t} \simeq \pi_{s} \widehat{M}_{s+t}, s, t \in \mathbb{Z}$. Pour tout $\widehat{M}^{*}$-module $N$ et tout entier $s$ on note $\Sigma^{s} N$ le $\widehat{M}^{*}$-module égal à $N^{t}$ en degré $s+t$, $t \in \mathbb{Z}$. Soit $X$ un espace profini; la filtration de $X$ par ses squelettes induit une filtration décroissante de la $M$-cohomologie continue de $X$, définie par $\mathrm{F}_{X}^{s} \widehat{M}^{*} X=$ $\operatorname{Ker}\left(\widehat{M}^{*} X \rightarrow \widehat{M}^{*} \mathrm{Sk}_{s-1} X\right)$. 
Proposition 2.1.9. - Soit $X$ un espace profini de dimension finie. Il existe une suite spectrale naturelle en $X$ et $M$ de terme $\mathrm{E}_{2}^{s, t}=\mathrm{H}^{s}\left(X ; \widehat{M}^{t}\right)$ convergeant fortement vers le gradué de la filtration de $\widehat{M}^{*} X$ induite par la filtration squelettale de $X$.

Démonstration. - Notons $X_{s}$ le $s$-ième squelette de $X$. La suite $X_{s-1+} \rightarrow X_{s+} \rightarrow$ $X_{s+} / X_{s-1+}$ induit en cohomologie un triangle exact

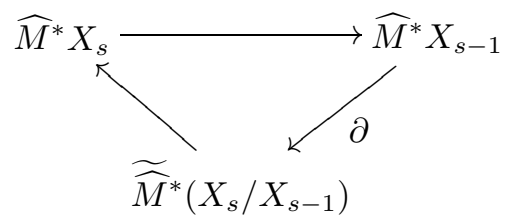

de sorte qu'on obtient une suite spectrale de terme $\mathrm{E}_{1}^{s, t}=\widetilde{\widehat{M}}^{s+t}\left(X_{s} / X_{s-1}\right)$ et dont la différentielle $\mathrm{d}_{r}$ est de bidegré $(r, 1-r)$. Si $X$ est de dimension $n$ alors la suite spectrale dégénère au terme $\mathrm{E}_{n+1}$ et $\Sigma^{s} \mathrm{E}_{n+1}^{s, *}$ s'identifie au quotient $\left(\mathrm{F}_{X}^{s} \widehat{M}^{*} X\right) /\left(\mathrm{F}_{X}^{s+1} \widehat{M}^{*} X\right)$. Il nous reste à identifier le terme $\mathrm{E}_{2}$.

L'espace profini $X_{s} / X_{s-1}$ est un pro-objet de la catégorie des bouquets finis de sphères de dimension $s$. La différentielle $\mathrm{d}_{1}: \widetilde{\widehat{M}}^{s+t}\left(X_{s} / X_{s-1}\right) \rightarrow \widetilde{\widehat{M}}^{s+t+1}\left(X_{s+1} / X_{s}\right)$ est induite par la composée $X_{s+1}^{+} / X_{s}^{+} \rightarrow \Sigma X_{s}^{+} \rightarrow \Sigma\left(X_{s}^{+} / X_{s-1}^{+}\right)$. Quitte à suspendre on peut supposer $s \geqslant 2$. On note pro-Ab la catégorie des pro-groupes abéliens. On dispose de morphismes canoniques

$$
\begin{aligned}
& \widetilde{\widehat{M}}^{s+t}\left(X_{s} / X_{s-1}\right) \longrightarrow \operatorname{Hom}_{\text {pro-h } \mathcal{S}_{\mathrm{pt}}}\left(X_{s} / X_{s-1}, \widehat{M}_{s+t}(-)\right) \\
& \longrightarrow \operatorname{Hom}_{\text {pro-Ab }}\left(\pi_{s}\left(X_{s} / X_{s-1}\right), \widehat{M}^{t}\right)
\end{aligned}
$$

et

$$
\begin{aligned}
\widetilde{\mathrm{H}}^{s}\left(X_{s} / X_{s-1}, \widehat{M}^{t}\right) & \longrightarrow \operatorname{Hom}_{\mathrm{pro}-\mathrm{h} \mathcal{S}_{\mathrm{pt}}}\left(X_{s} / X_{s-1}, \mathrm{~K}\left(\widehat{M}^{t}, s\right)\right) \\
& \longrightarrow \operatorname{Hom}_{\text {pro-Ab }}\left(\pi_{s}\left(X_{s} / X_{s-1}\right), \widehat{M}^{t}\right)
\end{aligned}
$$

LEMME 2.1.10. - Soient $n$ un entier supérieur ou égal à 2 et $S(-)$ un pro-bouquet fini de sphères de dimension $n$; alors la composée

$$
\begin{aligned}
\operatorname{Hom}_{\mathrm{h} \widehat{\mathcal{S}}_{\mathrm{pt}}}\left(S(-), \widehat{M}_{t}(-)\right) \longrightarrow \operatorname{Hom}_{\mathrm{pro}-\mathrm{h} \mathcal{S}}\left(S(-), \widehat{M}_{t}(-)\right) & \\
& \longrightarrow \operatorname{Hom}_{\text {pro-Ab }}\left(\pi_{n} S(-), \pi_{n} \widehat{M}_{t}(-)\right)
\end{aligned}
$$

est un isomorphisme de groupes abéliens.

Démonstration. — L'application

$$
\operatorname{Hom}_{\mathrm{h} \widehat{\mathcal{S}}_{\mathrm{pt}}}\left(S(i), \widehat{M}_{t}(j)\right) \longrightarrow \operatorname{Hom}_{\mathrm{pro}-\mathrm{Ab}}\left(\pi_{n} S(i), \pi_{n} \widehat{M}_{t}(j)\right)
$$


est un isomorphisme de groupes abéliens pour tout couple d'indices $(i, j)$. On en déduit que l'application

$$
\operatorname{Hom}_{\text {pro-h }} \mathcal{S}_{\mathrm{pt}}\left(S(-), \widehat{M}_{t}(-)\right) \longrightarrow \operatorname{Hom}_{\mathrm{pro}-\mathrm{Ab}}\left(\pi_{n} S(-), \pi_{n} \widehat{M}_{t}(-)\right)
$$

est un isomorphisme. Le pro-groupe abélien $\pi_{n+1} \widehat{M}_{t}(-)$ est pro-isomorphe à une tour de groupes finis donc pro-isomorphe à une tour de surjections. Comme chaque $\pi_{n} S(i)$ est un groupe abélien libre, le pro-groupe abélien

$$
\left(\operatorname{Hom}_{\text {pro-Ab }}\left(\pi_{n+1} \Sigma S(-), \pi_{n+1} \widehat{M}_{t}(j)\right)\right)_{j}
$$

est encore pro-isomorphe à une tour de surjections donc est sans $\lim ^{1}$. On en déduit que l'application

$$
\operatorname{Hom}_{\mathrm{h} \widehat{\mathcal{S}}_{\mathrm{pt}}}\left(S(-), \widehat{M}_{t}(-)\right) \longrightarrow \operatorname{Hom}_{\mathrm{pro}-\mathrm{h} \mathcal{S}_{\mathrm{pt}}}\left(S(-), \widehat{M}_{t}(-)\right)
$$

est un isomorphisme par le corollaire 1.4.3 et la proposition 1.3.2.

On conclut de ce qui précède que les groupes abéliens $\widetilde{\widehat{M}}^{s+t}\left(X_{s} / X_{s-1}\right)$ et $\widetilde{\mathrm{H}}^{s}\left(X_{s} / X_{s-1}, \widehat{M}^{t}\right)$ sont naturellement isomorphes (comme foncteurs en un probouquet fini de sphères de dimension $s$ ) puis que le terme $\mathrm{E}_{2}^{s, t}$ de la suite spectrale est naturellement isomorphe au s-ième groupe de cohomologie continue de $X$ à coefficients dans $\widehat{M}^{t}$.

Remarque. - Lorsque l'espace profini $X$ n'est pas de dimension finie, la suite spectrale d'Atiyah-Hirzebruch est toujours définie mais ne converge pas nécessairement vers le gradué de la filtration de $\widehat{M}^{*} X$ induite par la filtration squelettale de $X$. Adams ([Ad2, part.II, $\S 7,8]$ ) décrit les conditions de convergence par l'équivalence des deux conditions suivantes (le cadre profini ne changeant rien) :

(1) Pour tout couple d'entiers $(s, t)$ les applications $\mathrm{e}_{\infty}^{s, t} \rightarrow \lim _{r} \mathrm{E}_{r}^{s, t}$ et $\widehat{M}^{t} X \rightarrow$ $\lim _{s^{\prime}} \widehat{M}^{t} X / \mathrm{F}_{X}^{s^{\prime}}$ sont des isomorphismes.

(2) Pour tout couple d'entiers $(s, t)$ le groupe $\lim _{r}^{1} \mathrm{E}_{r}^{s, t}$ est nul.

(Où $\mathrm{e}_{\infty}^{s, *-s}$ désigne le quotient $\mathrm{F}_{X}^{s} \widehat{M}^{*} X / \mathrm{F}_{X}^{s+1} \widehat{M}^{*} X$.) La suite spectrale converge en particulier lorsqu'elle dégénère. Voir [BK, chap. IX, sect.5] pour une démonstration.

Revenons à la démonstration de la proposition 2.1.8. Soit $X$ un espace profini de dimension finie et sans $p$-torsion. Notons $\mathrm{E}_{r}^{s, t}(M, X)$ le terme $\mathrm{E}_{r}$ de la suite spectrale d'Atiyah-Hirzebruch associée à $X$ et une théorie cohomologique $M$. L'unité du spectre en anneau $\mathrm{HZ}$ induit un morphisme de spectres $\mathrm{MU} \rightarrow \mathrm{HZ} \wedge \mathrm{MU}$. L'homomorphisme entre anneaux de coefficients $\widehat{\mathrm{MU}}^{*} \rightarrow(\mathrm{HZ} \wedge \mathrm{MU})^{*}$ est injectif. On en déduit que pour $X$ sans $p$-torsion, l'application entre groupes bigradués $\mathrm{E}_{2}^{*, *}(\mathrm{MU}, X) \rightarrow \mathrm{E}_{2}^{*, *}(\mathrm{HZ} \wedge \mathrm{MU}, X)$ est encore injective. Or la suite spectrale d'AtiyahHirzebruch associée au spectre $\mathrm{HZ} \wedge \mathrm{MU}$ dégénère au terme $\mathrm{E}_{2}$ (car $\mathrm{HZ} \wedge \mathrm{MU}$ est somme de suspensions du spectre $\mathrm{HZ}$ ). Il en est donc de même de la suite spectrale associée à MU. 
On vérifie facilement à l'aide du lemme 2.1.2 et de la suite exacte longue en cohomologie associée à une suite exacte courte de coefficients que le $\mathrm{MU}^{*}$-module $\mathrm{H}^{s}\left(X, \widehat{\mathrm{MU}}^{*}\right)$ muni de la filtration par les coefficients est dans $\widehat{\mathcal{L}}$ pour tout $s$. On en déduit par récurrence et avec la proposition 2.1.3 que le quotient $\widehat{\mathrm{MU}}^{*} X / \mathrm{F}_{X}^{s}$, muni de la filtration par les coefficients, est dans $\widehat{\mathcal{L}}$ donc que $\widehat{\mathrm{MU}}^{*} X$ est dans $\widehat{\mathcal{L}}$ et est isomorphe au $\mathrm{MU}^{*}$-module $\oplus_{s} \Sigma^{s} \mathrm{H}^{s}\left(X, \widehat{\mathrm{MU}}^{*}\right)$. L'application $\widehat{\mathrm{MU}}^{*} X \rightarrow \mathrm{H}^{*} X$ se factorise par l'isomorphisme $\widehat{\mathrm{MU}}^{*} X \rightarrow \oplus_{s} \Sigma^{s} \mathrm{H}^{s}\left(X, \widehat{\mathrm{MU}}^{*}\right)$ donc induit un isomorphisme $\widehat{\mathrm{MU}}^{*} X / \mathrm{f}^{1} \rightarrow \mathrm{H}^{*} X$.

L'implication de (i) par (ii) est donnée par $[\mathbf{C S}]: X$ étant de dimension finie, supposons que $\mathrm{HZ}_{p}{ }^{*} X$ n'est pas sans torsion et soit $x$ un élément non nul et de torsion de $\mathrm{HZ}_{p}{ }^{*} X$ de degré $s_{0}$ maximal. Pour tout entier $s>s_{0}$ le terme $\mathrm{E}_{2}^{s, *}(\mathrm{MU}, X)$ s'injecte dans $\mathrm{E}_{2}^{s, *}(\mathrm{HZ} \wedge \mathrm{MU}, X)$. Comme la suite spectrale associée au spectre $\mathrm{HZ} \wedge \mathrm{MU}$ dégénère au terme $\mathrm{E}_{2}$, le morphisme $\mathrm{E}_{r}^{s, *}(\mathrm{MU}, X) \rightarrow \mathrm{E}_{r}^{s, *}(\mathrm{HZ} \wedge \mathrm{MU}, X)$ reste injectif pour $s>s_{0}$. L'élément $x$ donne un élément de torsion dans le terme $\mathrm{E}_{2}^{s_{0}, 0}(\mathrm{MU}, X)$ qui ne peut être dans l'image d'une différentielle pour une question de degré et qui est dans le noyau de toutes les différentielles par comparaison avec la suite spectrale associée à $\mathrm{H} \mathbb{Z} \wedge \mathrm{MU}$. Il se relève par récurrence en un élément de torsion de $\widehat{\mathrm{MU}}^{*} X / \mathrm{F}_{X}^{s}$, $s \geqslant s_{0}$, ce qui permet de conclure.

Proposition 2.1.11. - Soit $X$ un espace profini sans p-torsion; alors la MUcohomologie continue de $X$ munie de la filtration limite est dans $\widehat{\mathcal{L}}$ et l'application canonique $\widehat{\mathrm{MU}}^{*} X / \mathrm{f}^{1} \rightarrow \mathrm{H}^{*} X$ est un isomorphisme. De plus l'application $\widehat{\mathrm{MU}}^{*} X \rightarrow \lim _{s} \widehat{\mathrm{MU}}^{*} \mathrm{Sk}_{s} X$ est un isomorphisme dans $\mathcal{M}_{\mathrm{f}}$.

Démonstration. — La proposition est conséquence du lemme suivant :

Lemme 2.1.12. - Soit $\left(M_{s}\right)$ une tour d'objets de $\widehat{\mathcal{L}}$ telle que la tour de $\mathbb{F}_{p^{-}}$ espaces vectoriels gradués $\left(M_{s} / \mathrm{f}^{1}\right)$ est sans $\lim ^{1}$; alors la tour de $\mathrm{MU}^{*}$-modules $\left(M_{s}\right)$ est sans $\lim ^{1}$, le $\mathrm{MU}^{*}$-module $M_{\infty}=\lim _{s} M_{s}$ muni de la filtration limite $\mathrm{f}^{n} M_{\infty}=\bigcap_{s} \operatorname{Ker}\left(M_{\infty} \rightarrow M_{s} / \mathrm{f}^{n}\right)$ est dans $\widehat{\mathcal{L}}$ et l'application $M_{\infty} / \mathrm{f}^{n} \rightarrow \lim _{s} M_{s} / \mathrm{f}^{n}$ est un isomorphisme pour tout entier $n$.

Démonstration. - Notons pour tout entier $n M_{\infty, n}$ la limite en $s$ de la tour $\left(M_{s} / \mathrm{f}^{n}\right)$. La condition (3) du lemme 2.1.2 appliquée aux objets $M_{s}$ et l'absence de lim ${ }^{1}$ montrent que la tour $\left(M_{\infty, n}\right)$ est une tour de surjections. Par commutation des limites entre elles, $M_{\infty}$ s'identifie à la limite des $M_{\infty, n}$ et $\mathrm{f}^{n} M_{\infty}$ au noyau du morphisme $M_{\infty} \rightarrow$ $M_{\infty, n}$ lequel est surjectif par ce qui précède. Le fait que chaque $M_{s}$ vérifie les conditions (2) et (3) du lemme 2.1.2 impliquent alors que $M_{\infty}$ vérifie les mêmes conditions donc est dans $\widehat{\mathcal{L}}$.

Il reste à vérifier que la tour de $\mathrm{MU}^{*}$-modules $\left(M_{s}\right)$ est sans $\lim ^{1}$; or cela vient de la suite exacte $0 \rightarrow \lim _{n}^{1} M_{\infty, n} \rightarrow \lim _{s}^{1} M_{s} \rightarrow \lim _{n} \lim _{s}^{1}\left(M_{s} / \mathrm{f}^{n}\right) \rightarrow 0$ (proposition C.7) et du fait que l'annulation du terme $\lim _{s}^{1}\left(M_{s} / \mathrm{f}^{1}\right)$, la suite exacte 
$\mathrm{f}^{n} \mathrm{MU}^{*} / \mathrm{f}^{n+1} \mathrm{MU}^{*} \otimes M_{s} / \mathrm{f}^{1} \rightarrow M_{s} / \mathrm{f}^{n+1} \rightarrow M_{s} / \mathrm{f}^{n} \rightarrow 0$ et l'exactitude à droite de $\lim ^{1}$ impliquent par récurrence l'annulation du terme $\lim _{s}^{1}\left(M_{s} / \mathrm{f}^{n}\right)$.

Revenons à la démonstration de la proposition. Le $\mathrm{MU}^{*}$-module filtré $\widehat{\mathrm{MU}}^{*} \mathrm{Sk}_{s} X$ est dans $\widehat{\mathcal{L}}$ par le lemme 2.1.7 et la proposition 2.1.8. Comme la tour $\left(\left(\widehat{\mathrm{MU}}^{*} \mathrm{Sk}_{s} X\right) / \mathrm{f}^{1}\right)_{s}$ est stationnaire degré par degré car isomorphe à la tour $\left(\mathrm{H}^{*} \mathrm{Sk}_{s} X\right)_{s}$, le lemme montre que la tour des MU-cohomologies continues des squelettes $\mathrm{Sk}_{s} X$ est sans $\lim ^{1}$, que le $\mathrm{MU}^{*}$-module $\lim _{s} \widehat{\mathrm{MU}}^{*} \mathrm{Sk}_{s} X$ muni de la filtration limite est dans $\widehat{\mathcal{L}}$ et que l'application $\left(\lim _{s} \widehat{\mathrm{MU}^{*}} \mathrm{Sk}_{s} X\right) / \mathrm{f}^{1} \rightarrow \lim _{s}\left(\widehat{\mathrm{MU}}^{*} \mathrm{Sk}_{s} X\right) / \mathrm{f}^{1}$ est un isomorphisme. La proposition 1.6.2 montre alors que le morphisme de $\widehat{\mathrm{MU}}^{*}$-modules filtrés $\widehat{\mathrm{MU}}^{*} X \rightarrow \lim _{s} \widehat{\mathrm{MU}}^{*} \mathrm{Sk}_{s} X$ est un isomorphisme d'ensembles gradués, donc un isomorphisme dans $\mathcal{M}_{\mathrm{f}}$ par définition de la filtration de $\widehat{\mathrm{MU}}^{*} X$.

REMARque. - Soit $X$ un espace profini sans $p$-torsion; on peut montrer que la filtration limite de $\widehat{\mathrm{MU}}^{*} X$ coïncide avec la fermeture squelettale de la filtration par les coefficients de $\widehat{\mathrm{MU}}^{*} X$ définie par

$$
\mathrm{f}^{n} \widehat{\mathrm{MU}}^{*} X=\cap_{s}\left(\mathrm{f}^{n} \mathrm{MU}^{*} \cdot \widehat{\mathrm{MU}}^{*} X+\mathrm{F}_{X}^{s} \widehat{\mathrm{MU}}^{*} X\right) .
$$

Produit d'espaces sans p-torsion. — Soient $M$ et $N$ deux objets de $\widehat{\mathcal{L}}$ tels que $M / \mathrm{f}^{1}$ et $N / \mathrm{f}^{1}$ sont nuls en degré assez petit. Le $\mathrm{MU}^{*}$-module filtré complet $M \widehat{\otimes} N$ défini par $\forall n,(M \widehat{\otimes} N) / \mathrm{f}^{n}=M / \mathrm{f}^{n} \otimes_{\mathrm{MU}^{*}} N / \mathrm{f}^{n}$ est un objet de $\widehat{\mathcal{L}}$ qu'on appelle produit tensoriel de $M$ et $N$ dans $\widehat{\mathcal{L}}$.

Pour $X$ et $Y$ deux espaces profinis les projections de $X \times Y$ sur chacun des facteurs induisent un morphisme de $\widehat{\mathrm{MU}}^{*}$-algèbres de la somme $\widehat{\mathrm{MU}}^{*} X \otimes \widehat{\mathrm{MU}}^{*} \widehat{\mathrm{MU}}^{*} Y$ dans la MU-cohomologie continue du produit $X \times Y$, donc, pour tout entier $n$, un morphisme

$$
\left(\widehat{\mathrm{MU}}^{*} X\right) / \mathrm{f}^{n} \otimes_{\mathrm{MU}^{*}}\left(\widehat{\mathrm{MU}}^{*} Y\right) / \mathrm{f}^{n} \longrightarrow\left(\widehat{\mathrm{MU}}^{*}(X \times Y)\right) / \mathrm{f}^{n} .
$$

Proposition 2.1.13. - Soient $X$ et $Y$ deux espaces profinis sans p-torsion; alors leur produit $X \times Y$ est sans $p$-torsion et les applications

$$
\left(\widehat{\mathrm{MU}}^{*} X\right) / \mathrm{f}^{n} \otimes_{\mathrm{MU}^{*}}\left(\widehat{\mathrm{MU}}^{*} Y\right) / \mathrm{f}^{n} \longrightarrow\left(\widehat{\mathrm{MU}}^{*}(X \times Y)\right) / \mathrm{f}^{n}
$$

induisent un isomorphisme

$$
\widehat{\mathrm{MU}}^{*} X \widehat{\otimes} \widehat{\mathrm{MU}}^{*} Y \stackrel{\sim}{\longrightarrow} \widehat{\mathrm{MU}}^{*}(X \times Y)
$$

dans $\widehat{\mathcal{L}}$.

Démonstration. - On dispose sans hypothèse sur $X$ et $Y$ d'un isomorphisme canonique $\mathrm{H}^{*} X \otimes \mathrm{H}^{*} Y \rightarrow \mathrm{H}^{*}(X \times Y)$. On en déduit, par récurrence sur $n$ en utilisant la suite exacte longue en cohomologie associée à la suite exacte de coefficients $0 \rightarrow \mathbb{Z} / p \rightarrow \mathbb{Z} / p^{n+1} \rightarrow \mathbb{Z} / p^{n} \rightarrow 0$ et le fait que $X$ et $Y$ sont sans $p$-torsion, que l'application $\left(\mathrm{HZ} / p^{n}\right)^{*} X \otimes\left(\mathrm{H} \mathbb{Z} / p^{n}\right)^{*} Y \rightarrow\left(\mathrm{HZ} / p^{n}\right)^{*}(X \times Y)$ est un isomorphisme, puis que l'application $\left(\mathrm{HZ} / p^{n}\right)^{*}(X \times Y) \rightarrow \mathrm{H}^{*}(X \times Y)$ est surjective pour tout $n$. Donc $X \times Y$ est sans $p$-torsion. 
On dispose alors d'un morphisme $\widehat{\mathrm{MU}}^{*} X \widehat{\otimes} \widehat{\mathrm{MU}}^{*} Y \rightarrow \widehat{\mathrm{MU}}^{*}(X \times Y)$ dans $\widehat{\mathcal{L}}$. Puisque les morphismes

$$
\left(\widehat{\mathrm{MU}}^{*} X \widehat{\otimes} \widehat{\mathrm{MU}}^{*} Y\right) / \mathrm{f}^{1} \simeq\left(\widehat{\mathrm{MU}}^{*} X\right) / \mathrm{f}^{1} \otimes_{\mathrm{MU}^{*}}\left(\widehat{\mathrm{MU}}^{*} Y\right) / \mathrm{f}^{1} \longrightarrow \mathrm{H}^{*} X \otimes \mathrm{H}^{*} Y
$$

et $\widehat{\mathrm{MU}}^{*}(X \times Y) / \mathrm{f}^{1} \rightarrow \mathrm{H}^{*}(X \times Y)$ sont des isomorphismes, c'est un isomorphisme modulo $\mathrm{f}^{1}$ donc un isomorphisme dans $\widehat{\mathcal{L}}$ par le corollaire 2.1.4.

La proposition admet une version pointée : si $X$ et $Y$ sont deux espaces profinis pointés, ils sont naturellement rétracts du produit $X \times Y$ de sorte qu'on a une suite exacte courte

$$
0 \longrightarrow{\widetilde{\mathrm{MU}^{*}}}^{*}(X \wedge Y) \longrightarrow \widetilde{\widetilde{\mathrm{MU}}^{*}}(X \times Y) \longrightarrow \widetilde{\widehat{\mathrm{MU}}}^{*}(X \vee Y) \longrightarrow 0 .
$$

On en déduit :

Proposition 2.1.14. - Soient $X$ et $Y$ deux espaces profinis pointés sans p-torsion alors $X \wedge Y$ est sans p-torsion et on a un isomorphisme naturel

$$
\widetilde{\mathrm{MU}^{*}} X \widehat{\otimes} \widetilde{\mathrm{MU}^{*}} Y \stackrel{\sim}{\longrightarrow} \widetilde{\widehat{\mathrm{MU}}}^{*}(X \wedge Y) .
$$

Généralisation : Le produit d'une famille quelconque d'espaces profinis est la limite filtrante des produits des sous-familles finies. Si les espaces profinis sont sans $p$-torsion, leur produit l'est également par ce qui précède et le lemme suivant :

Lemme 2.1.15. - Soit $\left(X_{\alpha}\right)$ un diagramme filtrant d'espaces profinis sans p-torsion; alors l'espace profini $\lim _{\alpha} X_{\alpha}$ est sans p-torsion.

Démonstration. - La cohomologie modulo $p^{n}$ continue de l'espace profini $\lim _{\alpha} X_{\alpha}$ est la colimite des cohomologies modulo $p^{n}$ continues des $X_{\alpha}$ (lemme 1.1.1). L'exactitude à droite des colimites permet de conclure.

Vers le cas général. - On note $\mathcal{E}$ ns-gr la catégorie des ensembles $\mathbb{Z}$-gradués et, pour tout entier $n,\{n\}$ l'ensemble gradué formé du seul singleton en degré $n$.

Soit $X$ un espace profini; on dispose par définition d'une bijection naturelle $\operatorname{Hom}_{\mathrm{h} \widehat{\mathcal{S}}}\left(X, \widehat{\mathrm{MU}}_{n}\right) \simeq \operatorname{Hom}_{\mathcal{E n s} \text {-gr }}\left(\{n\}, \widehat{\mathrm{MU}}^{*} X\right)$. Pour tout ensemble gradué $S$ définissons $\mathrm{K}(S)$ comme le produit dans $\widehat{\mathcal{S}}$ des espaces profinis $\widehat{\mathrm{MU}}_{|s|}, s$ décrivant $S$ et $|s|$ désignant son degré. L'espace profini $\mathrm{K}(S)$ est sans $p$-torsion et on dispose d'un morphisme $S \rightarrow \widehat{\mathrm{MU}}^{*} \mathrm{~K}(S)$ induisant une bijection

$$
\operatorname{Hom}_{\mathrm{h} \widehat{\mathcal{S}}}(X, \mathrm{~K}(S)) \longrightarrow \operatorname{Hom}_{\mathcal{E n s}-\mathrm{gr}}\left(S, \widehat{\mathrm{MU}}^{*} X\right) \text {. }
$$

L'adjonction munit l' endofoncteur $\mathcal{E}$ ns-gr $\rightarrow \mathcal{E}$ ns-gr, $S \mapsto \widehat{\mathrm{MU}}^{*} \mathrm{~K}(S)$, qu'on note G, d'une structure de monade, i.e. de transformations naturelles $\mathrm{Id} \rightarrow \mathrm{G}$ (l'unité de l'adjonction) et $\mathrm{G} \circ \mathrm{G} \rightarrow \mathrm{G}$ (qui associe à un ensemble gradué $S$ l'image en $\mathrm{MU}$ cohomologie continue de l'application adjointe de l'identité de $\mathrm{G}(S)$ ) vérifiant les axiomes d'un monoïde (voir l'appendice A). Observons que $\mathrm{G}$ et la transformation naturelle $\mathrm{G} \circ \mathrm{G} \rightarrow \mathrm{G}$ sont à valeurs dans $\widehat{\mathcal{L}}$. L'application $X \rightarrow \mathrm{K}\left(\widehat{\mathrm{MU}}^{*} X\right)$, adjointe de 
l'identité de $\widehat{\mathrm{MU}}^{*} X$, induit en MU-cohomologie continue un morphisme $\mathrm{G}\left(\widehat{\mathrm{MU}}^{*} X\right) \rightarrow$ $\widehat{\mathrm{MU}}^{*} X$ faisant de $\widehat{\mathrm{MU}}^{*} X$ une G-algèbre (une G-algèbre est un ensemble gradué $S$ muni d'un morphisme $\mathrm{G}(S) \rightarrow S$ vérifiant les axiomes d'une action).

La structure de monade sur G permet d'associer à la MU-cohomologie continue de $X$ un objet simplicial G. $\left(\widehat{\mathrm{MU}}^{*} X\right)$, image en MU-cohomologie continue d'un diagramme d'espaces sans $p$-torsion, et un morphisme de $\mathrm{G} .\left(\widehat{\mathrm{MU}}^{*} X\right)$ dans l'objet simplicial constant $\widehat{\mathrm{MU}}^{*} X$ qui est une équivalence d'homotopie entre objets simpliciaux de $\mathcal{E}$ ns-gr (voir la section A.4). En particulier la MU-cohomologie continue de $X$ apparaît canoniquement comme le coégalisateur dans $\mathcal{E}$ ns-gr d'une double-flèche avec section commune de $\widehat{\mathcal{L}}$.

Nous étudierons dans les prochaines sections la structure additive qui résulte de cette présentation. La structure de G-algèbre de $\widehat{\mathrm{MU}}^{*} X$ est ce que nous appellerons la structure d'algèbre instable de $\widehat{\mathrm{MU}}^{*} X$. Elle sera étudiée en section 3 .

\section{2. $M U^{*}$-modules filtrés à présentation libre et $M U$-cohomologie continue des espaces profinis dans le cas général}

1-complexes de $\widehat{\mathcal{L}}$ et $\widehat{\mathrm{L}}$-algèbres. - A tout objet $S$ de $\mathcal{E}$ ns-gr on associe le $\mathrm{MU}^{*}$-module libre $\mathrm{L}(S)$ de base $S$ et son complété $\widehat{\mathrm{L}}(S)$ pour la filtration par les coefficients. Le foncteur $\widehat{\mathrm{L}}: \mathcal{E}$ ns-gr $\rightarrow \mathcal{M}_{\mathrm{fc}}$ est adjoint à gauche du foncteur oubli $\mathcal{M}_{\mathrm{fc}} \rightarrow \mathcal{E}$ ns-gr ( $c f$. section 2.1). On en déduit une structure de monade sur l'endofoncteur de $\mathcal{E}$ ns-gr composé de $\widehat{\mathrm{L}}$ et du foncteur oubli, qu'on note encore $\widehat{\mathrm{L}}$.

On note $\widehat{\mathcal{M}}$ la catégorie des $\widehat{\mathrm{L}}$-algèbres de $\mathcal{E}$ ns-gr.

Le coégalisateur dans $\mathcal{E}$ ns-gr d'un 1-complexe de $\widehat{L}$-algèbres est un coégalisateur scindé par la proposition A.1.2; autrement dit la monade $\widehat{\mathrm{L}}$ vérifie la condition $\left(\mathcal{Q}_{0}\right)$ (voir l'appendice $\mathrm{A}$ ). On en déduit que la catégorie $\widehat{\mathcal{M}}$ possède toutes les colimites indexées par une catégorie petite et que l'oubli $\widehat{\mathcal{M}} \rightarrow \mathcal{E}$ ns-gr commute aux coégalisateurs de 1-complexes (proposition A.1.5). Le foncteur $\widehat{L}$ transforme toute somme finie d'ensembles gradués en le produit des ensembles gradués images donc $\widehat{\mathcal{M}}$ est abélienne par la proposition A.3.2. Un morphisme de $\widehat{\mathcal{M}}$ est un monomorphisme (respectivement un épimorphisme) si et seulement si l'application d'ensembles gradués sous-jacente est injective (respectivement surjective) degré par degré ( $c f$. l'appendice A).

Notons O l'oubli $\mathcal{M}_{\mathrm{fc}} \rightarrow \mathcal{E}$ ns-gr. On dispose du foncteur $\widetilde{\mathrm{O}}: \mathcal{M}_{\mathrm{fc}} \rightarrow \widehat{\mathcal{M}}$ relevant $\mathrm{O}$, qui associe à un $\widehat{\mathrm{MU}}^{*}$-module filtré $M$ de $\mathcal{M}_{\mathrm{fc}}$ l'ensemble gradué sous-jacent à $M$ muni du morphisme $\widehat{\mathrm{L}}(M) \rightarrow M$ adjoint de l'identité de $M$. Ce foncteur induit une équivalence de catégories entre $\widehat{\mathcal{L}}$ et la sous-catégorie pleine de $\widehat{\mathcal{M}}$ formée des $\widehat{\mathrm{L}}$ algèbres libres (lemme A.2.1). Nous identifierons ces deux catégories par la suite.

Toute $\widehat{L}$-algèbre libre est un objet projectif de $\widehat{\mathcal{M}}$ ( $c f$. la section A.3 de l'appendice). Toute $\widehat{\mathrm{L}}$-algèbre $M$ est un quotient de la $\widehat{\mathrm{L}}$-algèbre libre $\widehat{\mathrm{L}}(M)$. Si $M$ est un objet projectif de $\widehat{\mathcal{M}}$ alors $M$ est un facteur direct de $\widehat{\mathrm{L}}(M)$ donc est dans $\widehat{\mathcal{L}}$ par la proposition 2.1.3. 
$\widehat{\mathrm{L}}$-algèbres, structure de $\mathrm{MU}^{*}$-module et filtration. - Nous développons dans ce paragraphe les liens entre la catégorie $\widehat{\mathcal{M}}$ et les catégories $\mathcal{M}$ et $\mathcal{M}_{\mathrm{f}}$.

L'oubli de la filtration $\widehat{\mathrm{L}}(\mathcal{E}$ ns-gr $) \cong \widehat{\mathcal{L}} \rightarrow \mathcal{M}$ s'entend canoniquement en un foncteur $\mathrm{O}^{a}: \widehat{\mathcal{M}} \rightarrow \mathcal{M}$ tel que pour toute $\widehat{\mathrm{L}}$-algèbre $M, \mathrm{O}^{a} M$ est le coégalisateur du 1-complexe de $\mathrm{MU}^{*}$-modules $\widehat{\mathrm{L}}^{2}(M) \leftrightarrows \widehat{\mathrm{L}}(M)$ (proposition A.2.2) : $\mathrm{O}^{a} M$ est le $M^{*}$-module sous-jacent à $M$. Comme tout objet de $\widehat{\mathcal{L}}$ est complet pour la filtration $p$-adique, le $\mathrm{MU}^{*}$-module sous-jacent à une $\widehat{\mathrm{L}}$-algèbre est Ext-p-complet (i.e. dans l'image du foncteur $\operatorname{Ext}\left(\mathbb{Z} / p^{\infty},-\right)$, voir [BK, chap. VI]). En particulier sa structure de $\mathrm{MU}^{*}$-module s'étend de façon naturelle en une structure de $\widehat{\mathrm{MU}}^{*}$-module.

Comme les catégories $\widehat{\mathcal{M}}$ et $\mathcal{M}$ sont celles des algèbres associées à des monades sur Ens-gr, la proposition A.3.3 montre que $\mathrm{O}^{a}$ est additif, exact et admet un adjoint à gauche qu'on note $\widehat{\mathrm{L}}^{a}$. ( $\mathrm{Si} M$ est un $\mathrm{MU}^{*}$-module libre alors $\widehat{\mathrm{L}}^{a}(M)$ s'identifie par construction à l'objet $\widehat{M}$ de $\widehat{\mathcal{L}}$, complété de $M$ pour la filtration par les coefficients.) L'adjonction munit la composée de $\widehat{\mathrm{L}}^{a}$ avec le foncteur oubli d'une structure de monade sur $\mathcal{M}$, qu'on note encore $\widehat{\mathrm{L}}^{a}$. L'oubli $\mathrm{O}^{a}$ se relève en un foncteur $\left(\mathrm{O}^{a}\right) \widetilde{\mathcal{M}} \rightarrow \mathcal{M}\left(\widehat{\mathrm{L}}^{a}\right)$ ( $c f$. appendice A.2) qui est une équivalence de catégories par cette même proposition.

On note $\mathcal{M}^{0}$ et $\widehat{\mathcal{M}}^{0}$ les sous-catégories pleines de $\mathcal{M}$ et $\widehat{\mathcal{M}}$ formées des objets nuls en degré assez grand.

Proposition 2.2.1. - La restriction de la monade $\widehat{\mathrm{L}}^{a} \grave{a} \mathcal{M}^{0}$ est un idempotent de $\mathcal{M}^{0}$ d'image la sous-catégorie pleine formée des $\mathrm{MU}^{*}$-modules Ext-p-complets. En particulier le foncteur oubli $\widehat{\mathcal{M}}^{0} \rightarrow \mathcal{M}^{0}$ induit une équivalence de catégories entre $\widehat{\mathcal{M}}^{0}$ et la sous-catégorie pleine de $\mathcal{M}^{0}$ formée des modules Ext-p-complets.

Démonstration. - Le foncteur $\left(\mathrm{O}^{a}\right)^{\sim}$ induit encore un isomorphisme entre $\widehat{\mathcal{M}}^{0}$ et $\mathcal{M}^{0}\left(\widehat{\mathrm{L}}^{a}\right)$. Soit $M$ un objet de $\mathcal{M}^{0}$; le $\mathrm{MU}^{*}$-module $\widehat{\mathrm{L}}^{a}(M)$ s'identifie au complété de $M$ pour la filtration $p$-adique si $M$ est libre donc au Ext-p-complété de $M$ en général. On en déduit que le morphisme $\eta(M): M \rightarrow \widehat{\mathrm{L}}^{a}(M)$ est l'identité si et seulement si $M$ est Ext-p-complet, en particulier si $M$ est dans l'image de $\widehat{\mathrm{L}}^{a}$, donc que $\mu(M)$ et $\widehat{\mathrm{L}}^{a}(\eta(M))$ sont toujours l'identité $(\mu(M)$ est un inverse à gauche de $\eta\left(\widehat{\mathrm{L}}^{a}(M)\right)$ et de $\left.\widehat{\mathrm{L}}^{a}(\eta(M))\right)$. En particulier, si $M$ est Ext- $p$-complet, l'identité de $M$ fait de $M$ une $\widehat{\mathrm{L}}^{a}$-algèbre. Il est clair qu'un morphisme de $\widehat{\mathrm{MU}}^{*}$-modules entre $\widehat{\mathrm{MU}}^{*}$ modules Ext- $p$-complets est alors un morphisme de $\widehat{\mathrm{L}}^{a}$-algèbres. Inversement soit $M$ une $\widehat{\mathrm{L}}^{a}$-algèbre nulle en degré assez grand; alors le 1-complexe $\left(\widehat{\mathrm{L}}^{a}\right)^{2}(M) \leftrightarrows \widehat{\mathrm{L}}^{a}(M)$ est le 1-complexe constant $\widehat{\mathrm{L}}^{a}(M) \leftrightarrows \widehat{\mathrm{L}}^{a}(M)$ donc $\widehat{\mathrm{L}}^{a}(M) \rightarrow M$ est un isomorphisme donc $M$ est Ext-p-complet et $\widehat{\mathrm{L}}^{a}(M) \rightarrow M$ est l'identité de $M$.

Parallèlement l'inclusion $\iota: \widehat{\mathrm{L}}(\mathcal{E}$ ns-gr $) \cong \widehat{\mathcal{L}} \rightarrow \mathcal{M}_{\mathrm{f}}$ s'étend canoniquement en un foncteur $\widetilde{\iota}: \widehat{\mathcal{M}} \rightarrow \mathcal{M}_{\mathrm{f}}:$ Pour $M$ une $\widehat{\mathrm{L}}$-algèbre, $\widetilde{\iota} M$ est le $\mathrm{MU}^{*}$-module sous-jacent à $M$ muni de la filtration héritée de celle de $\widehat{\mathrm{L}}(M)$. Le foncteur $\widetilde{\iota}$ est additif et exact à 
droite par la proposition A.3.1, mais $\mathcal{M}_{\mathrm{f}}$ n'est pas la catégorie des algèbres associées à une monade sur $\mathcal{E}$ ns-gr.

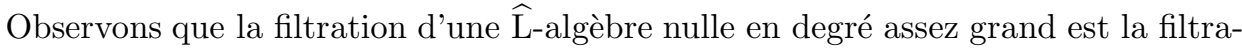
tion par les coefficients. Observons également que le foncteur $\widetilde{\iota}$ commute aux produits, mais il n'est pas exact à gauche.

Lemme 2.2.2. - Pour tout entier $n$ le foncteur $\widehat{\mathcal{M}} \rightarrow \mathcal{M}, M \mapsto M / \mathrm{f}^{n}$ commute aux colimites.

Démonstration. - Il suffit d'après la proposition A.1.6 de vérifier que pour tout diagramme d'ensembles gradués $\left(S_{\alpha}\right)$ l'application $\left.\widehat{\mathrm{L}}\left(\operatorname{colim}_{\alpha} S_{\alpha}\right) / \mathrm{f}^{n} \rightarrow \operatorname{colim}_{\alpha} \widehat{\mathrm{L}}\left(S_{\alpha}\right)\right) / \mathrm{f}^{n}$ est un isomorphisme. Or pour tout ensemble gradué $S, \widehat{\mathrm{L}}(S) / \mathrm{f}^{n}$ est le $\mathrm{MU}^{*} / \mathrm{f}^{n}$-module libre sur $S$, ce qui entraîne le résultat.

Lemme 2.2.3. - Soit $M$ une $\widehat{\mathrm{L}}$-algèbre; alors $M$ est nulle si et seulement si le $\mathbb{F}_{p^{-}}$espace vectoriel gradué $M / \mathrm{f}^{1}$ est nul.

Démonstration. - Choisissons une présentation $M_{1} \rightarrow M_{0} \rightarrow M$ de $M$ dans $\widehat{\mathcal{L}}$. Si $M / \mathrm{f}^{1}$ est nul alors l'application $M_{1} / \mathrm{f}^{1} \rightarrow M_{0} / \mathrm{f}^{1}$ est surjective en chaque degré par le lemme ci-dessus, donc l'application $M_{1} \rightarrow M_{0}$ est surjective en chaque degré par la proposition 2.1.3 donc $M$ est nul.

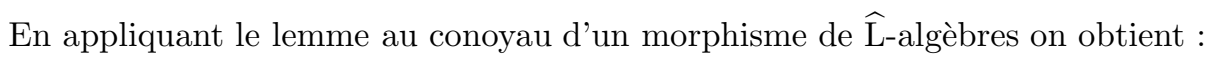

Corollaire 2.2.4. - Soit $M \rightarrow N$ un morphisme de $\widehat{\mathrm{L}}$-algèbres; alors $M \rightarrow N$ est surjective en chaque degré si et seulement si l'application $M / \mathrm{f}^{1} \rightarrow N / \mathrm{f}^{1}$ est surjective en chaque degré.

Lemme 2.2.5. - Soit $M$ une $\widehat{\mathrm{L}}$-algèbre, alors $\widetilde{\iota M}$ se surjecte sur son complété pour la filtration.

Démonstration. - $\widetilde{\iota} M$ est le coégalisateur dans $\mathcal{M}_{\mathrm{f}}$ du diagramme $\widehat{\mathrm{L}}^{2}(M) \leftrightarrows \widehat{\mathrm{L}}(M)$. Notons $K_{n}$ le $\mathrm{MU}^{*}$-module image de $\mathrm{d}_{0}-\mathrm{d}_{1}: \widehat{\mathrm{L}}^{2}(M) / \mathrm{f}^{n} \rightarrow \widehat{\mathrm{L}}(M) / \mathrm{f}^{n}$. Les $K_{n}$ forment une tour de surjections et la suite $0 \rightarrow \mathrm{K}_{n} \rightarrow \widehat{\mathrm{L}}(M) / \mathrm{f}^{n} \rightarrow M / \mathrm{f}^{n} \rightarrow 0$ est exacte donc l'application $\widehat{\mathrm{L}}(M) \rightarrow \lim _{n} M / \mathrm{f}^{n}$ est surjective, or celle-ci se factorise par l'application $\widehat{\mathrm{L}}(M) \rightarrow M$.

Rappelons qu'on dispose du foncteur $\widetilde{\mathrm{O}}: \mathcal{M}_{\mathrm{fc}} \rightarrow \widehat{\mathcal{M}}$ relevant l'oubli $\mathcal{M}_{\mathrm{fc}} \rightarrow \mathcal{E}$ ns-gr et pour tout objet $N$ de $\mathcal{M}_{\mathrm{fc}}$ du morphisme canonique $\widetilde{\iota} \widetilde{\mathrm{O}}(N) \rightarrow N$ qui est l'identité dans $\mathcal{M}$ mais pas dans $\mathcal{M}_{\mathrm{f}}$ en général. Le morphisme de structure $\widehat{\mathrm{L}}(\widetilde{\mathrm{O}}(N)) \rightarrow \widetilde{\mathrm{O}}(N)$ est caractérisé par le fait que c'est un morphisme dans $\mathcal{M}_{\mathrm{f}}$ puisqu'il est alors adjoint de l'identité de l'ensemble gradué $N$; autrement dit il existe une seule structure de

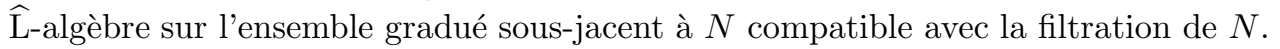


Nous dirons que la filtration d'un objet $M$ de $\mathcal{M}_{\mathrm{f}}$ est fermante ou pour abréger que $M$ est f-filtré si l'inclusion $\mathrm{f}^{n} \mathrm{MU}^{*} \cdot M+\mathrm{f}^{n+1} M \subset \mathrm{f}^{n} M$ est une bijection pour tout entier $n$. (Cette condition est équivalente à la condition (2) du lemme 2.1.2.)

Exemples. - Le complété $\widehat{M}$ d'un MU*-module $M$ est f-filtré; le coégalisateur dans $\mathcal{M}_{\mathrm{f}}$ d'un 1-complexe de $\mathrm{MU}^{*}$-modules f-filtrés est f-filtré.

On note $\mathcal{M}_{\mathrm{f}-\mathrm{fc}}$ la sous-catégorie pleine de $\mathcal{M}_{\mathrm{f}}$ formée des $\mathrm{MU}^{*}$-modules f-filtrés complets et $\widehat{\mathcal{M}}_{c}$ la sous-catégorie pleine de $\widehat{\mathcal{M}}$ formée des $\widehat{\mathrm{L}}$-algèbres complètes pour la filtration.

Lemme 2.2.6. - Soit $M$ un objet de $\mathcal{M}_{\mathrm{fc}}$, alors $\widetilde{\iota} \widetilde{\mathrm{O}}(M)$ est dans $\mathcal{M}_{\mathrm{f}-\mathrm{fc}}$.

Démonstration. - Il s'agit de vérifier que le $\mathrm{MU}^{*}$-module filtré $\widetilde{\iota} \widetilde{\mathrm{O}}(M)$ est complet. On sait déjà qu'il se surjecte sur son complété pour la filtration par le lemme 2.2.5. D'autre part la filtration de $M$ induite par celle de $\widehat{\mathrm{L}}(M)$ est plus fine que la filtration originelle de $M$ donc est séparée.

Pour tout objet $M$ de $\widehat{\mathcal{L}}$ le diagramme

$$
\widehat{\mathrm{L}}^{2}(M) \rightleftarrows \widehat{\mathrm{L}}(M) \longrightarrow M
$$

est scindé dans $\widehat{\mathcal{L}}$ (lemme A.2.1 (b)) de sorte que le morphisme $\widetilde{\iota} \widetilde{\mathrm{O}}(M) \rightarrow \iota(M)$ est l'identité dans $\mathcal{M}_{\mathrm{f}}$. Plus généralement on a le

Lemme 2.2.7. - Soit $M \rightarrow N$ un morphisme de $\mathcal{M}_{\mathrm{f}-\mathrm{fc}}$ qui est un isomorphisme de $\mathrm{MU}^{*}$-modules; alors $M \rightarrow N$ est un isomorphisme de $\mathcal{M}_{\mathrm{f}-\mathrm{fc}}$.

Démonstration. - On introduit comme pour la démonstration du point (a) de la proposition 2.1.3 le noyau $K_{n}$ de la surjection $M / \mathrm{f}^{n} \rightarrow N / \mathrm{f}^{n}$. Le fait que $M$ et $N$ sont f-filtrés implique que la tour des $K_{n}$ est une tour de surjections. Le fait que l'application $M \rightarrow N$ est bijective implique que la limite des $K_{n}$ est nulle donc chacun des $K_{n}$ est nul.

Proposition 2.2.8. - Le foncteur $\widetilde{\iota} \widetilde{\mathrm{O}}: \mathcal{M}_{\mathrm{fc}} \rightarrow \mathcal{M}_{\mathrm{fc}}$ est un idempotent d'image $\mathcal{M}_{\mathrm{f}-\mathrm{fc}}$ et $\widetilde{\iota}: \widehat{\mathcal{M}} \rightarrow \mathcal{M}_{\mathrm{f}}$ induit une équivalence de catégories entre $\widehat{\mathcal{M}}_{c}$ et la catégorie $\mathcal{M}_{\mathrm{f}-\mathrm{fc}}$.

Démonstration. - La restriction de $\widetilde{\iota}$ à $\widehat{\mathcal{M}}_{c}$ est à valeur dans $\mathcal{M}_{\mathrm{fc}}$ par définition de

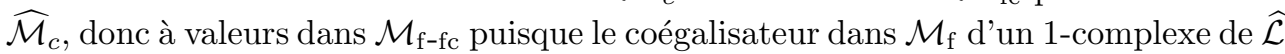
est f-filtré. Le lemme 2.2.6 montre par ailleurs que le foncteur $\widetilde{\mathrm{O}}$ est à valeur dans $\widehat{\mathcal{M}}_{c}$. Pour tout objet $M$ de $\widehat{\mathcal{M}}_{c}$ le diagramme coégalisateur $\widehat{\mathrm{L}}^{2}(M) \leftrightarrows \widehat{\mathrm{L}}(M) \rightarrow M$ induit un morphisme $M \rightarrow \widetilde{\mathrm{O}} \widetilde{\iota} M$ qui est l'identité dans $\mathcal{E}$ ns-gr donc l'identité dans $\widehat{\mathcal{M}}$. D'autre part pour tout objet $M$ de $\mathcal{M}_{\mathrm{f}-\mathrm{fc}}$ le morphisme $\widetilde{\iota} \widetilde{\mathrm{O}}(M) \rightarrow M$ est un isomorphisme dans $\mathcal{M}$ donc également dans $\mathcal{M}_{\mathrm{f}-\mathrm{fc}}$ par le lemme 2.2.7, d'où le résultat. 


\section{EXEMPLE ET REMARQUE}

- Pour toute $\widehat{\mathrm{L}}$-algèbre $M$ et tout entier $n$, le $\mathrm{MU}^{*}$-module $M / \mathrm{f}^{n}$ muni de la filtration par les coefficients est f-filtré complet et la surjection $M \rightarrow M / \mathrm{f}^{n}$ en fait

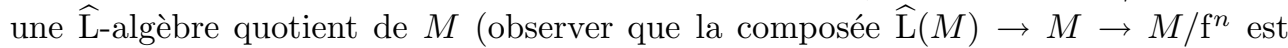
un morphisme de $\mathcal{M}_{\mathrm{f}-\mathrm{fc}}$ donc de $\widehat{\mathcal{M}}$ et utiliser la discussion sur les objets quotients en fin de section A.1 de l'appendice). En particulier le $\mathrm{MU}^{*}$-module $\mathrm{MU}^{*} / \mathrm{f}^{n}$ muni de la filtration par les coefficients est une $\widehat{\mathrm{L}}$-algèbre quotient de l'objet $\widehat{\mathrm{MU}}^{*} \in \widehat{\mathcal{L}}$. Observons que le foncteur $\widehat{\mathcal{M}} \rightarrow \mathcal{M}, M \mapsto M / \mathrm{f}^{n}$ commute aux sommes infinies mais pas le foncteur $\widehat{\mathcal{M}} \rightarrow \widehat{\mathcal{M}}, M \mapsto M / \mathrm{f}^{n}$.

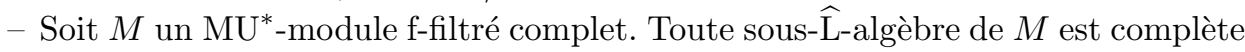
pour sa filtration car elle est séparée pour la filtration induite par celle de $M$ et se surjecte sur son complété (lemme 2.2.5). Par contre elle n'est pas nécessairement fermée dans $M$ pour la topologie de $M$.

Observons que le foncteur $\widetilde{\mathrm{O}}: \mathcal{M}_{\mathrm{fc}} \rightarrow \widehat{\mathcal{M}}$ commute aux limites. En particulier si la limite dans $\mathcal{M}_{\text {fc }}$ d'un diagramme d'objets de $\widehat{\mathcal{L}}$ est dans $\widehat{\mathcal{L}}$, cette limite coïncide avec la limite dans $\widehat{\mathcal{M}}$ du diagramme ( $c f$. le lemme 2.1.12 pour une condition suffisante).

Filtration squelettale d'une $\widehat{\mathrm{L}}$-algèbre. — Rappelons qu'une sous- $\widehat{\mathrm{L}}$-algèbre d'une $\widehat{\mathrm{L}}$ algèbre $M$ (respectivement une $\widehat{\mathrm{L}}$-algèbre quotient) est un sous-ensemble gradué (respectivement un ensemble gradué quotient) muni d'une structure de $\widehat{L}$-algèbre compatible (voir dans l'appendice la fin de la section A.1).

Soient $L$ un objet de $\widehat{\mathcal{L}}$ et $n$ un entier. On note $\mathrm{F}^{n} L$ la sous- $\widehat{\mathrm{L}}$-algèbre de $L$ engendrée par les éléments de degré supérieur ou égal à $n$; c'est un facteur direct de $L$ naturel en $L$. La suite des $\mathrm{F}^{n} L$ forme une filtration décroissante complète de $L$ dans $\widehat{\mathcal{M}}$.

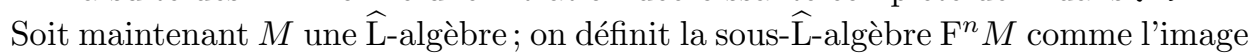
dans $M$ de $\mathrm{F}^{n} \widehat{\mathrm{L}}(M)$ de sorte que le quotient $M / \mathrm{F}^{n} M$, qu'on note pour abréger $M / \mathrm{F}^{n}$,

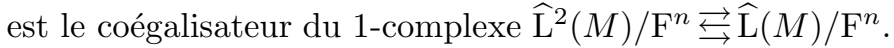

Le foncteur $\widehat{\mathcal{M}} \rightarrow \widehat{\mathcal{M}}, M \mapsto M / \mathrm{F}^{n}$ est additif et exact à droite (proposition A.3.1). Tout morphisme de $M$ dans une $\widehat{\mathrm{L}}$-algèbre nulle en degré supérieur ou égal à $n$ se factorise (de façon unique) par le quotient $M / \mathrm{F}^{n}$. L'application $M / \mathrm{f}^{1} \rightarrow\left(M / \mathrm{F}^{n}\right) / \mathrm{f}^{1}$ est un isomorphisme en degré inférieur strictement à $n$ et est nulle en degré supérieur ou égal à $n$.

La suite des $\mathrm{F}^{n} M$ forme une filtration décroissante, pas nécessairement complète

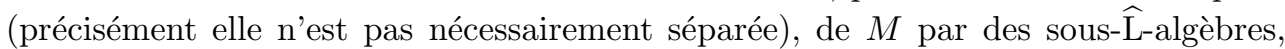
qu'on appelle la filtration squelettale de $M$.

LEMME 2.2.9. - Soit $M$ une $\widehat{\mathrm{L}}$-algèbre admettant une présentation $M_{1} \rightarrow M_{0} \rightarrow M$ dans $\widehat{\mathcal{L}}$ telle que $M_{1} / \mathrm{f}^{1}$ et $M_{0} / \mathrm{f}^{1}$ sont finis en chaque degré; alors la filtration squelettale de $M$ est complète. 
Démonstration. - Pour tout entier $s$ les $\mathbb{Z}_{p}$-modules gradués sous-jacents à $M_{1} / \mathrm{F}^{s}$ et $M_{0} / \mathrm{F}^{s}$ sont de type fini en chaque degré, donc également le $\mathbb{Z}_{p}$-module gradué sousjacent au noyau qu'on note $K_{s}$ du morphisme $M_{1} / \mathrm{F}^{s} \rightarrow M_{0} / \mathrm{F}^{s}$. On en déduit que la tour $\left(K_{s}\right)$ est sans $\lim ^{1}$ puis que la suite $\lim _{s} M_{1} / \mathrm{F}^{s} \rightarrow \lim _{s} M_{0} / \mathrm{F}^{s} \rightarrow \lim _{s} M / \mathrm{F}^{s} \rightarrow 0$ est exacte, ce qui permet de conclure.

Produit tensoriel dans $\widehat{\mathcal{M}}$. - Nous revenons d'abord sur le produit tensoriel de deux objets de $\widehat{\mathcal{L}}$ : On définit le produit tensoriel $M \widehat{\otimes} N$ de $\operatorname{deux}$ objets $M$ et $N$ de $\widehat{\mathcal{L}}$ comme le $\mathrm{MU}^{*}$-module filtré complet donné par

$$
(M \widehat{\otimes} N) / \mathrm{f}^{n}=\lim _{s}\left(\left(M / \mathrm{F}^{s}\right) / \mathrm{f}^{n} \otimes_{\mathrm{MU}^{*}}\left(N / \mathrm{F}^{s}\right) / \mathrm{f}^{n}\right)
$$

(donc $(M \widehat{\otimes} N) / \mathrm{f}^{1}$ en degré $k$ est le produit $\left.\prod_{i+j=k}\left(M / \mathrm{f}^{1}\right)^{i} \otimes_{\mathbb{Z} / p}\left(N / \mathrm{f}^{1}\right)^{j}\right) ;$ alors $M \widehat{\otimes} N$ est dans $\widehat{\mathcal{L}}$ et coïncide avec le produit tensoriel de $M$ et $N$ défini en section 1.1.1 si $M / \mathrm{f}^{1}$ et $N / \mathrm{f}^{1}$ sont nuls en degré assez petit. On a de plus des isomorphismes canoniques $\widehat{\mathrm{MU}}^{*} \widehat{\otimes} M \simeq M \simeq M \widehat{\otimes} \widehat{\mathrm{MU}}^{*}$.

Les morphismes $M \otimes_{\widehat{\mathrm{MU}}^{*}} N \rightarrow M / \mathrm{f}^{n} \otimes_{\mathrm{MU}^{*}} N / \mathrm{f}^{n}$ induisent un morphisme de $\widehat{\mathrm{MU}}^{*}$ modules $M \otimes \widehat{\mathrm{MU}}^{*} N \rightarrow M \widehat{\otimes} N$.

LEMme 2.2.10. - Soient $M$ et $N$ deux objets de $\widehat{\mathcal{L}}$, alors le morphisme $M \otimes \widehat{\mathrm{MU}}^{*} N \rightarrow$ $M \widehat{\otimes} N$ est un isomorphisme (de $\widehat{\mathrm{MU}}^{*}{ }^{-}$modules) si et seulement si l'une des conditions suivantes est satisfaite :

(1) $M$ et $N$ sont nuls en degré assez grand et $M / \mathrm{f}^{1}$ ou $N / \mathrm{f}^{1}$ est fini en chaque degré.

(2) $M / \mathrm{f}^{1}$ ou $N / \mathrm{f}^{1}$ est fini.

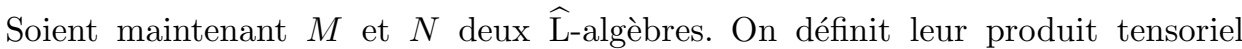
$M \widehat{\otimes} N$ comme le coégalisateur du 1-complexe $\widehat{\mathrm{L}}^{2}(M) \widehat{\otimes} \widehat{\mathrm{L}}^{2}(N) \leftrightarrows \widehat{\mathrm{L}}(M) \widehat{\otimes} \widehat{\mathrm{L}}(N)$ défini par les morphismes $\mathrm{d}_{0} \widehat{\otimes} \mathrm{d}_{0}, \mathrm{~d}_{1} \widehat{\otimes} \mathrm{d}_{1}$ et $\mathrm{s}_{0} \widehat{\otimes} \mathrm{s}_{0}$. On a des isomorphismes canoniques $M \widehat{\otimes} \widehat{M U}^{*} \simeq M, M \widehat{\otimes} N \simeq N \widehat{\otimes} M, M \widehat{\otimes}(N \widehat{\otimes} P) \simeq(M \widehat{\otimes} N) \widehat{\otimes} P$ pour $M, N$ et $P$ dans $\widehat{\mathcal{M}}$.

On vérifie que pour tout objet $L$ de $\widehat{\mathcal{L}}$ le foncteur $L \widehat{\otimes}-: \widehat{\mathcal{L}} \rightarrow \widehat{\mathcal{L}}$ commute aux produits finis, de sorte que pour toute $\widehat{\mathrm{L}}$-algèbre $M$, le foncteur $M \widehat{\otimes}-: \widehat{\mathcal{M}} \rightarrow \widehat{\mathcal{M}}$ est additif et exact à droite ( $c f$. la proposition A.3.1). Il ne commute pas aux sommes infinies en général.

On dispose d'un morphisme canonique de $\widehat{\mathrm{MU}}^{*}$-modules $M \otimes \widehat{\mathrm{MU}}^{*} N \rightarrow M \widehat{\otimes} N$. Le lemme 2.2.10 et l'exactitude à droite du produit tensoriel entraînent :

Proposition 2.2.11. - Soient $M$ et $N$ deux $\widehat{\mathrm{L}}$-algèbres nulles en degré assez grand. On suppose que $N$ est le conoyau d'un morphisme $N_{1} \rightarrow N_{0}$ de $\widehat{\mathcal{L}}$ tel que $N_{1} / \mathrm{f}^{1}$ et $N_{0} / \mathrm{f}^{1}$ sont finis en chaque degré; alors l'application $M \otimes_{\widehat{\mathrm{MU}}^{*}} N \rightarrow M \widehat{\otimes} N$ est un isomorphisme. 


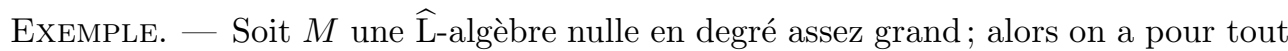
entier $n$ un isomorphisme canonique $M \widehat{\otimes} \mathrm{MU}^{*} / \mathrm{f}^{n} \simeq M / \mathrm{f}^{n}$. Nous verrons en fin de section 4.1 que ce résultat est vrai quelque soit $M$.

Proposition 2.2.12. - Soient $M$ et $N$ deux $\widehat{\mathrm{L}-a l g e ̀ b r e s . ~ O n ~ s u p p o s e ~ q u e ~ l ' u n e ~ d e s ~}$ conditions suivantes est satisfaite :

(1) $M$ et $N$ sont nulles en degré assez grand.

(2) $N$ est le conoyau d'un morphisme $N_{1} \rightarrow N_{0}$ de $\widehat{\mathcal{L}}$ tel que $N_{1} / \mathrm{f}^{1}$ et $N_{0} / \mathrm{f}^{1}$ sont nuls sauf en un nombre fini de degrés.

Alors on a pour tout entier $n$ un isomorphisme canonique

$$
(M \widehat{\otimes} N) / \mathrm{f}^{n} \simeq M / \mathrm{f}^{n} \otimes_{\mathrm{MU}^{*}} N / \mathrm{f}^{n} .
$$

Démonstration. - Le résultat est vrai par définition pour $M$ et $N$ dans $\widehat{\mathcal{L}}$ tels que $M / \mathrm{f}^{1}$ et $N / \mathrm{f}^{1}$ sont nuls en degré assez grand ou si $N / \mathrm{f}^{1}$ est concentré en un nombre fini de degrés. Le cas général s'en déduit par exactitude à droite du produit tensoriel.

Notons, pour $S$ un ensemble gradué, $\Sigma S$ l'ensemble gradué égal à $S^{k-1}$ en chaque degré $k$. Le foncteur $S \mapsto \Sigma S$ est un isomorphisme de la catégorie $\mathcal{E}$ ns-gr dans ellemême.

On dispose d'un isomorphisme canonique $\widehat{\mathrm{L}}(\Sigma S) \cong \Sigma \widehat{\mathrm{L}}(S)$ dans Ens-gr compatible avec la structure de monade de $\widehat{\mathrm{L}}$. Soit alors $M$ une $\widehat{\mathrm{L}}$-algèbre; la composée $\widehat{\mathrm{L}}(\Sigma M) \cong \Sigma \widehat{\mathrm{L}}(M) \rightarrow \Sigma M$, respectivement $\widehat{\mathrm{L}}\left(\Sigma^{-1} M\right) \cong \Sigma^{-1} \widehat{\mathrm{L}}(M) \rightarrow \Sigma^{-1} M$, munit $\Sigma M$, respectivement $\Sigma^{-1} M$, d'une structure de $\widehat{\mathrm{L}}$-algèbre naturelle en $M \in \widehat{\mathcal{M}}$. Les endofoncteurs $\Sigma$ et $\Sigma^{-1}$ de $\mathcal{E}$ ns-gr se relèvent donc en des endofoncteurs de $\widehat{\mathcal{M}}$ inverses l'un de l'autre.

On vérifie que la $\widehat{\mathrm{L}}$-algèbre $\Sigma M$ s'identifie au produit tensoriel de $M$ avec la $\widehat{\mathrm{L}}$ algèbre libre en un générateur de degré 1 . Plus généralement le produit tensoriel $\widehat{\otimes}$ sur $\widehat{\mathcal{M}}$ coïncide avec le produit tensoriel $\otimes_{\widehat{\mathrm{L}}}$ défini dans l'appendice B.

Présentation de la MU-cohomologie continue des espaces profinis. - Rappelons que pour $S$ un ensemble gradué on définit l'espace profini $\mathrm{K}(S)$ comme le produit des $\widehat{\mathrm{MU}}_{|s|}, s$ décrivant $S . \mathrm{K}(S)$ est sans $p$-torsion et on dispose d'une bijection

$$
\operatorname{Hom}_{\mathrm{h} \widehat{\mathcal{S}}}(X, \mathrm{~K}(S)) \simeq \operatorname{Hom}_{\mathcal{E n s}-\mathrm{gr}}\left(S, \widehat{\mathrm{MU}}^{*} X\right)
$$

naturelle en $X \in \mathrm{h} \widehat{\mathcal{S}}$ et $S \in \mathcal{E}$ ns-gr. On en déduit une structure de monade sur le foncteur $\mathrm{G}: \mathcal{E}$ ns-gr $\rightarrow \mathcal{E}$ ns-gr, $S \mapsto \widehat{\mathrm{MU}}^{*} \mathrm{~K}(S)$ et une structure de G-algèbre sur l'ensemble gradué $\widehat{\mathrm{MU}}^{*} X$. On dispose donc pour tout espace profini $X$ d'un diagramme d'ensembles gradués

$$
\mathrm{G}^{2}\left(\widehat{\mathrm{MU}}^{*} X\right) \rightleftarrows \mathrm{G}\left(\widehat{\mathrm{MU}}^{*} X\right) \longrightarrow \widehat{\mathrm{MU}}^{*} X
$$

(cf. l'appendice A), en fait diagramme de $\mathcal{M}_{\mathrm{f}}$ car image en MU-cohomologie continue du diagramme

$$
X \longrightarrow \mathrm{K}\left(\widehat{\mathrm{MU}}^{*} X\right) \rightleftarrows \mathrm{K}\left(\mathrm{G}\left(\widehat{\mathrm{MU}}^{*} X\right)\right)
$$


de $h \widehat{\mathcal{S}}$. (Voir la section 2.1 pour la définition de la filtration de la MU-cohomologie continue d'un espace profini.)

Ce diagramme vérifie :

- le 1-complexe $\mathrm{G}^{2}\left(\widehat{\mathrm{MU}}^{*} X\right) \leftrightarrows \mathrm{G}\left(\widehat{\mathrm{MU}}^{*} X\right)$ de $\mathcal{M}_{\mathrm{f}}$ est un 1-complexe de $\widehat{\mathcal{L}}$;

- le diagramme d'ensembles gradués sous-jacent $\mathrm{G}^{2}\left(\widehat{\mathrm{MU}}^{*} X\right) \leftrightarrows \mathrm{G}\left(\widehat{\mathrm{MU}}^{*} X\right) \rightarrow$ $\widehat{\mathrm{MU}}^{*} X$ est un diagramme coégalisateur scindé.

Supposons que la filtration de $\widehat{\mathrm{MU}}^{*} X$ est fermante et complète - c'est le cas si $X$ est de dimension finie ou s'il est sans $p$-torsion - alors $\widehat{\mathrm{MU}}^{*} X$ est une $\widehat{\mathrm{L}}$-algèbre et $\mathrm{G}\left(\widehat{\mathrm{MU}}^{*} X\right) \rightarrow \widehat{\mathrm{MU}}^{*} X$ est un morphisme de $\widehat{\mathrm{L}}$-algèbres (par la proposition 2.2.8). Le diagramme $\mathrm{G}^{2}\left(\widehat{\mathrm{MU}}^{*} X\right) \rightrightarrows \mathrm{G}\left(\widehat{\mathrm{MU}}^{*} X\right) \rightarrow \widehat{\mathrm{MU}}^{*} X$ est coégalisateur dans $\widehat{\mathcal{M}}$ puisqu'il est coégalisateur dans $\mathcal{E}$ ns-gr, de sorte que la structure de $\widehat{\mathrm{L}}$-algèbre de $\widehat{\mathrm{MU}}^{*} X$ coïncide avec celle héritée de $\mathrm{G}\left(\widehat{\mathrm{MU}}^{*} X\right)$.

Pour $X$ quelconque, on définit une structure de $\widehat{\mathrm{L}}$-algèbre sur $\widehat{\mathrm{MU}}^{*} X$ comme celle héritée de $\mathrm{G}\left(\widehat{\mathrm{MU}}^{*} X\right)$. La filtration de $\widehat{\mathrm{MU}}^{*} X$ donnée par sa structure de $\widehat{\mathrm{L}}$-algèbre (donc celle héritée de $\mathrm{G}\left(\widehat{\mathrm{MU}}^{*} X\right)$ ) est a priori plus fine que celle définie en section 2.1. Nous désignons désormais par $\mathrm{f}^{n} \widehat{\mathrm{MU}}^{*} X$ (respectivement $\widehat{\mathrm{MU}}^{*} X / \mathrm{f}^{n}$ ) le $n$-ième terme de la filtration associée à la structure de $\widehat{\mathrm{L}}$-algèbre de $\widehat{\mathrm{MU}}^{*} X$ (respectivement le quotient associé).

Soient $X$ et $Y$ deux espaces profinis; on dispose du diagramme de $\mathrm{h} \widehat{\mathcal{S}}$

$$
X \times Y \longrightarrow \mathrm{K}\left(\widehat{\mathrm{MU}}^{*} X\right) \times \mathrm{K}\left(\widehat{\mathrm{MU}}^{*} Y\right) \rightleftarrows \mathrm{K}\left(\mathrm{G}\left(\widehat{\mathrm{MU}}^{*} X\right)\right) \times \mathrm{K}\left(\mathrm{G}\left(\widehat{\mathrm{MU}}^{*} Y\right)\right) .
$$

La formule de Künneth (proposition 2.1.13) pour la MU-cohomologie continue d'un produit d'espaces profinis sans $p$-torsion fournit un isomorphisme entre les 1-complexes de $\widehat{\mathcal{L}}$

$$
\mathrm{G}^{2}\left(\widehat{\mathrm{MU}}^{*} X\right) \widehat{\otimes} \mathrm{G}^{2}\left(\widehat{\mathrm{MU}}^{*} Y\right) \rightleftarrows \mathrm{G}\left(\widehat{\mathrm{MU}}^{*} X\right) \widehat{\otimes} \mathrm{G}\left(\widehat{\mathrm{MU}}^{*} Y\right)
$$

et

$$
\widehat{\mathrm{MU}}^{*}\left(\mathrm{~K}\left(\mathrm{G}\left(\widehat{\mathrm{MU}}^{*} X\right)\right) \times \mathrm{K}\left(\mathrm{G}\left(\widehat{\mathrm{MU}}^{*} Y\right)\right)\right) \rightleftarrows \widehat{\mathrm{MU}}^{*}\left(\mathrm{~K}\left(\widehat{\mathrm{MU}}^{*} X\right) \times \mathrm{K}\left(\widehat{\mathrm{MU}}^{*} X\right)\right)
$$

donc un morphisme $\widehat{\mathrm{MU}}^{*} X \widehat{\otimes} \widehat{\mathrm{MU}}^{*} Y \rightarrow \widehat{\mathrm{MU}}^{*}(X \times Y)$ qui n'est pas un isomorphisme en général. Nous construirons en section 4.2 la suite spectrale aboutissant à la MUcohomologie continue de $X \times Y$.

\section{Structure d'algèbre instable}

Nous revenons dans cette section sur la bijection naturelle

$$
\operatorname{Hom}_{\mathrm{h} \widehat{\mathcal{S}}}(X, \mathrm{~K}(S)) \simeq \operatorname{Hom}_{\mathcal{E n s}-\mathrm{gr}}\left(S, \widehat{\mathrm{MU}}^{*} X\right) .
$$

Nous notons $\mathrm{G}$ le foncteur $S \mapsto \widehat{\mathrm{MU}}^{*} \mathrm{~K}(S)$, appelons MU-algèbre instable un ensemble gradué muni d'une structure de G-algèbre et notons $\mathcal{K}_{\mathrm{MU}}$ la catégorie des MU-algèbres instables. La MU-cohomologie continue d'un espace profini en est l'objet type. Le foncteur $\mathrm{G}$ et la transformation naturelle $\mathrm{G} \circ \mathrm{G} \rightarrow \mathrm{G}$ sont à valeurs dans $\widehat{\mathcal{L}}$ 
de sorte qu'on obtient un foncteur oubli $\mathcal{K}_{\mathrm{MU}} \rightarrow \widehat{\mathcal{M}}$ commutant aux coégalisateurs de 1-complexes. Cet oubli admet un adjoint à gauche $\mathrm{G}^{a}$ et $\mathcal{K}_{\mathrm{MU}}$ s'identifie à la catégorie des $\mathrm{G}^{a}$-algèbres de $\widehat{\mathcal{M}}$.

Nous définissons une version réduite $\mathcal{K}_{\mathrm{MU}}$ - de $\mathcal{K}_{\mathrm{MU}}$ dont la MU-cohomologie continue réduite d'un espace est l'objet type et qui s'obtient comme la catégorie d'algèbres associée à une monade $\widetilde{\mathrm{G}}$ sur $\mathcal{E}$ ns-gr ou $\widetilde{\mathrm{G}}^{a}$ sur $\widehat{\mathcal{M}}$. On dispose d'un foncteur oubli $\mathcal{K}_{\mathrm{MU}} \rightarrow \mathcal{K}_{\mathrm{MU}-}$ au dessus de $\widehat{\mathcal{M}}$ et d'un adjoint $M \mapsto M_{+}$de cet oubli mimant l'adjonction entre $\mathrm{h} \widehat{\mathcal{S}}$ et $\mathrm{h} \widehat{\mathcal{S}}_{\mathrm{pt}}$.

Nous montrons que le produit tensoriel dans $\widehat{\mathcal{M}}$ se relève en la somme dans $\mathcal{K}_{\mathrm{MU}}$ de façon compatible avec la formule de Künneth en MU-cohomologie continue.

Dans le paragraphe 3.2 nous étudions le lien entre MU-algèbres instables et algèbres instables sur l'algèbre de Steenrod modulo $p$. On note $\mathcal{K}_{\mathrm{H}}$ la catégorie formée par ces dernières (voir par exemple $[\mathbf{S c}]$ ) et $\mathcal{E}$ la catégorie des $\mathbb{F}_{p}$-espaces vectoriels gradués. La catégorie $\mathcal{K}_{\mathrm{H}}$ apparaît comme la catégorie des $\mathrm{G}_{\mathrm{H}}$-algèbres de $\mathcal{E}$, où $\mathrm{G}_{\mathrm{H}}$ est une monade associée aux espaces d'Eilenberg-Mac Lane généralisés ([La2 $]$ ). Le foncteur $\mathcal{K}_{\mathrm{MU}} \rightarrow \mathcal{E}, M \mapsto M / \mathrm{f}^{1}$ se relève en un foncteur $\mathcal{K}_{\mathrm{MU}} \rightarrow \mathcal{K}_{\mathrm{H}}$ tel que le morphisme $\left(\widehat{\mathrm{MU}}^{*} X\right) / \mathrm{f}^{1} \rightarrow \mathrm{H}^{*} X$ est un morphisme d'algèbres instables.

Dans le dernier paragraphe (3.3) nous montrons que pour toute MU-algèbre instable $M$, l'ensemble $\operatorname{Hom}_{\mathcal{K}_{\mathrm{MU}}}\left(M, \widehat{\mathrm{MU}}^{*}\right)$ est en bijection avec l'ensemble $\operatorname{Hom}_{\mathcal{K}_{\mathrm{H}}}\left(M / \mathrm{f}^{1}\right.$, $\mathbb{Z} / p$ ) donc, si $M$ est la MU-cohomologie continue d'un espace profini fibrant $X$ sans $p$-torsion, avec l'ensemble $\pi_{0} X$. Ce résultat s'étend au cas $X$ fibrant quelconque.

\subsection{Espaces d'Eilenberg-Mac Lane généralisés et MU-algèbres instables}

On note $\mathcal{K}_{\mathrm{MU}}$ la catégorie des G-algèbres de $\mathcal{E}_{\text {ns-gr }}$ (voir la fin de la section 2.2 pour la définition de la monade $\mathrm{G}$ ). Les objets de $\mathcal{K}_{\mathrm{MU}}$ sont appelées $\mathrm{MU}$-algèbres instables. Voici deux exemples fondamentaux :

- L'image par G d'un ensemble gradué $S$ est la MU-algèbre instable libre sur $S$ : l'application $S \mapsto \mathrm{G}(S)$ induit une bijection $\operatorname{Hom}_{\mathcal{K}_{\mathrm{MU}}}(\mathrm{G}(S), N) \rightarrow \operatorname{Hom}_{\mathcal{E} \text { ns-gr }}(S, N)$ pour tout $N \in \mathcal{K}_{\mathrm{MU}}$.

- Soit $X$ un espace profini; l'image en MU-cohomologie continue de l'application canonique $X \rightarrow \mathrm{K}\left(\widehat{\mathrm{MU}}^{*} X\right)$ fait de la MU-cohomologie continue de $X$ une MU-algèbre instable fonctoriellement en $X$.

Pour tout objet $M$ de $\mathcal{K}_{\mathrm{MU}}$, le diagramme $\mathrm{G}^{2}(M) \leftrightarrows \mathrm{G}(M)$, dont $M$ est le coégalisateur dans $\mathcal{E}$ ns-gr, est un 1-complexe de $\widehat{\mathcal{L}}$. On en déduit une structure de $\widehat{\mathrm{L}}$-algèbre sur $M$, c'est à dire un foncteur oubli $\mathrm{O}^{a}: \mathcal{K}_{\mathrm{MU}} \rightarrow \widehat{\mathcal{M}}$. Comme le foncteur $\mathrm{G}$ et la transformation naturelle $\mathrm{G} \circ \mathrm{G} \rightarrow \mathrm{G}$ sont à valeurs dans la catégorie des groupes abéliens gradués, le coégalisateur dans $\mathcal{E}$ ns-gr d'un 1-complexe de G-algèbres est un coégalisateur scindé (proposition A.1.4). On en déduit l'existence dans $\mathcal{K}_{\mathrm{MU}}$ des colimites indexées par une catégorie petite et la commutation du foncteur oubli $\mathcal{K}_{\mathrm{MU}} \rightarrow \mathcal{E}$ ns-gr aux coégalisateurs de 1-complexes. Le corollaire A.2.6 montre que le 
foncteur $\mathrm{O}^{a}: \mathcal{K}_{\mathrm{MU}} \rightarrow \widehat{\mathcal{M}}$ commute aux coégalisateurs de 1-complexes, admet un adjoint à gauche qu'on note $\mathrm{G}^{a}$ et que le foncteur associé $\widetilde{\mathrm{O}}^{a}: \mathcal{K}_{\mathrm{MU}} \rightarrow \widehat{\mathcal{M}}\left(\mathrm{O}^{a} \mathrm{G}^{a}\right)$ est une équivalence de catégories. Le foncteur $\mathrm{O}^{a}$ ne commute pas aux coégalisateurs en général.

On note encore $\mathrm{G}^{a}$ la composée $\mathrm{O}^{a} \mathrm{G}^{a}$. Par construction, l'image par $\mathrm{G}^{a}$ de la $\widehat{\mathrm{L}}$ algèbre libre sur un ensemble gradué $S$ est la G-algèbre libre sur $S$. On en déduit que l'image par $\mathrm{G}^{a}$ d'un objet de $\widehat{\mathcal{L}}$ est dans $\widehat{\mathcal{L}}$.

Version réduite. - L'objet $\widehat{\mathrm{MU}}^{*}$, image par G de l'objet initial $\varnothing$ de $\mathcal{E}$ ns-gr, est un objet initial dans $\mathcal{K}_{\mathrm{MU}}$. Pour tout espace profini pointé $X$, l'application pt $\rightarrow X$ induit un morphisme $\widehat{\mathrm{MU}}^{*} X \rightarrow \widehat{\mathrm{MU}}^{*}$ rétract de l'unité $\widehat{\mathrm{MU}}^{*} \rightarrow \widehat{\mathrm{MU}}^{*} X$ dans $\mathcal{K}_{\mathrm{MU}}$ et de noyau $\widetilde{\mathrm{MU}}^{*} X$ dans $\widehat{\mathcal{M}}$ : l'algèbre instable $\widehat{\mathrm{MU}}^{*} X$ est augmentée.

Soit $S$ un ensemble gradué; l'espace profini $\mathrm{K}(S)$ est un produit d'espaces profinis pointés. La bijection $\operatorname{Hom}_{\mathrm{h} \widehat{\mathcal{S}}_{\mathrm{pt}}}\left(X, \widehat{\mathrm{MU}}_{s}\right) \simeq \widetilde{\widehat{\mathrm{MU}}^{s}} X, s \in \mathbb{Z}$, (voir la section 1.6) se généralise en une bijection $\operatorname{Hom}_{\mathrm{h}} \widehat{\mathcal{S}}_{\mathrm{pt}}(X, \mathrm{~K}(S)) \simeq \operatorname{Hom}_{\mathcal{E n s} \text {-gr }}\left(S, \widetilde{\widehat{\mathrm{MU}}}^{*} X\right)$ d'où on déduit une structure de monade sur le foncteur $\widetilde{\mathrm{G}}: \mathcal{E}$ ns-gr $\rightarrow \mathcal{E}$ ns-gr, $S \mapsto \widetilde{\widehat{\mathrm{MU}}} * \mathrm{~K}(S)$.

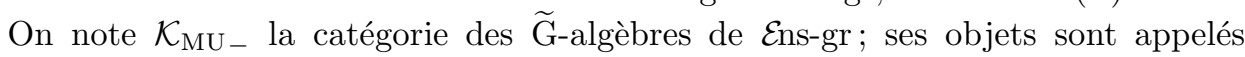
MU-algèbres instables non-unitaires. Si $X$ est un espace profini pointé, l'adjonction ci-dessus fait de $\widetilde{\widehat{\mathrm{MU}}}^{*} X$ un objet de $\mathcal{K}_{\mathrm{MU}-}$. Comme pour $\mathcal{K}_{\mathrm{MU}}$, le 1-complexe

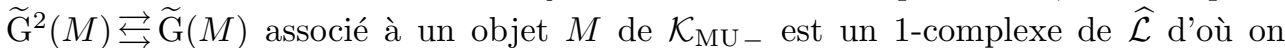
déduit un foncteur oubli $\mathcal{K}_{\mathrm{MU}-} \rightarrow \widehat{\mathcal{M}}$.

Soit $M$ dans $\mathcal{K}_{\mathrm{MU}}$. Le 1-complexe $\mathrm{G}^{2}(M) \leftrightarrows \mathrm{G}(M)$ est l'image en MU-cohomologie continue du 1-cocomplexe $\mathrm{K}(M) \leftrightarrows \mathrm{K}(\mathrm{G}(M))$ donc image en MU-cohomologie continue réduite du 1-cocomplexe $\mathrm{K}(M)_{+} \leftrightarrows \mathrm{K}(\mathrm{G}(M))_{+}$, donc est naturellement un 1complexe de $\mathcal{K}_{\mathrm{MU}_{-}}$. On en déduit un foncteur oubli $\mathcal{K}_{\mathrm{MU}} \rightarrow \mathcal{K}_{\mathrm{MU}}$ au dessus de l'identité de $\mathcal{E}$ ns-gr.

On note $\mathcal{K}_{\mathrm{MU}_{0}}$ la catégorie des algèbres instables augmentées, c'est-à-dire la catégorie des objets de $\mathcal{K}_{\mathrm{MU}}$ au dessus de $\widehat{\mathrm{MU}}^{*}$. Pour tout ensemble gradué $S$, le point base de $\mathrm{K}(S)$ fait de $\mathrm{G}(S)$ un objet de $\mathcal{K}_{\mathrm{MU}_{0}}$. On dispose d'un oubli évident $\mathcal{K}_{\mathrm{MU}_{0}} \rightarrow \mathcal{K}_{\mathrm{MU}}$.

Pour $M$ dans $\mathcal{K}_{\mathrm{MU}_{0}}$ on note $\widetilde{M}$ l'ensemble gradué sous-jacent au noyau dans $\widehat{\mathcal{M}}$ du morphisme de $M \rightarrow \widehat{\mathrm{MU}}^{*}$. On a une bijection naturelle $\operatorname{Hom}_{\mathcal{K}_{\mathrm{MU}}}(\mathrm{G}(S), M) \simeq$ $\operatorname{Hom}_{\mathcal{E} n \text { s-gr }}(S, \widetilde{M})$ d'où une structure de monade sur le foncteur $\mathcal{E}$ ns-gr $\rightarrow \mathcal{E}$ ns-gr, $S \mapsto(\mathrm{G}(S))^{\widetilde{C}}$ et une structure d'algèbre sur $\widetilde{M}$ relativement à cette monade. Cette monade coïncide avec $\widetilde{G}$ de sorte que $\widetilde{M}$ est dans $\mathcal{K}_{\mathrm{MU}-}$. Le foncteur $\mathcal{K}_{\mathrm{MU} 0} \rightarrow \mathcal{E}$ ns-gr, $M \mapsto \widetilde{M}$ admet $S \mapsto \mathrm{G}(S)$ pour adjoint à gauche, commute aux coégalisateurs de 1complexes et ne transforme que les isomorphismes en isomorphismes. Son relèvement $\mathcal{K}_{\mathrm{MU}_{0}} \rightarrow \mathcal{E} \operatorname{Ens-gr}(\widetilde{G})=\mathcal{K}_{\mathrm{MU}}$ - est donc une équivalence de catégories par la proposition A.1.6. On note $M_{+}$l'algèbre instable augmentée associée à une algèbre instable non-unitaire $M$ par cette équivalence de catégories. On a donc pour tout ensemble 
gradué $S$ un isomorphisme canonique $\widetilde{\mathrm{G}}(S)_{+} \cong \mathrm{G}(S)$ dans $\mathcal{K}_{\mathrm{MU}_{0}}$ et plus généralement un isomorphisme canonique $\left(\widetilde{\widehat{\mathrm{MU}}}^{*} X\right)_{+} \cong \widehat{\mathrm{MU}}^{*} X$ pour tout espace profini pointé $X$.

Observons que la composée $\mathcal{K}_{\mathrm{MU}} \stackrel{\sim}{\longrightarrow} \mathcal{K}_{\mathrm{MU}_{0}} \rightarrow \widehat{\mathcal{M}}, M \mapsto M_{+}$est isomorphe au foncteur $M \mapsto M \oplus \widehat{\mathrm{MU}}^{*}$ et pour tout espace profini $X$ l'image de $\widehat{\mathrm{MU}}^{*} X$ par la composée $\mathcal{K}_{\mathrm{MU}} \rightarrow \mathcal{K}_{\mathrm{MU}-} \stackrel{\sim}{\longrightarrow} \mathcal{K}_{\mathrm{MU}_{0}}$ s'identifie à $\widehat{\mathrm{MU}}^{*}\left(X_{+}\right)$. Par composition des adjonctions, la composée $\mathcal{K}_{\mathrm{MU}_{-}} \simeq \mathcal{K}_{\mathrm{MU}_{0}} \rightarrow \mathcal{K}_{\mathrm{MU}}, M \mapsto M_{+}$est adjoint à gauche du foncteur oubli $\mathcal{K}_{\mathrm{MU}} \rightarrow \mathcal{K}_{\mathrm{MU}-}$.

La description de $\mathcal{K}_{\mathrm{MU}}$ comme catégorie des $\mathrm{G}^{a}$-algèbres de $\widehat{\mathcal{M}}$ se mime dans $\mathcal{K}_{\mathrm{MU}-}$ : Pour toute $\widehat{\mathrm{L}}$-algèbre $M$, l'algèbre instable $\mathrm{G}^{a}(M)$ est augmentée par le morphisme $\mathrm{G}^{a}(M) \rightarrow \widehat{\mathrm{MU}}^{*}$ adjoint du morphisme nul $M \rightarrow \widehat{\mathrm{MU}}^{*}$ et on a une bijection naturelle $\operatorname{Hom}_{\mathcal{K}_{\mathrm{MU}_{0}}}\left(\mathrm{G}^{a}(M), N\right) \simeq \operatorname{Hom}_{\widehat{\mathcal{M}}}(M, \tilde{N})$. La composée $\widehat{\mathcal{M}} \rightarrow \widehat{\mathcal{M}}$, $M \mapsto\left(\mathrm{G}^{a}(M)\right)^{\sim}$, qu'on note $\widetilde{\mathrm{G}}^{a}$, a une structure de monade et la catégorie $\mathcal{K}_{\mathrm{MU}}$ s'identifie à la catégorie des $\widetilde{\mathrm{G}}^{a}$-algèbres de $\widehat{\mathcal{M}}$.

Observons que l'algèbre instable non unitaire nulle est l'objet initial et terminal de $\mathcal{K}_{\mathrm{MU}-}$. L'oubli $\mathcal{K}_{\mathrm{MU}-} \rightarrow \widehat{\mathcal{M}}$ commute aux limites. En particulier le noyau dans $\widehat{\mathcal{M}}$

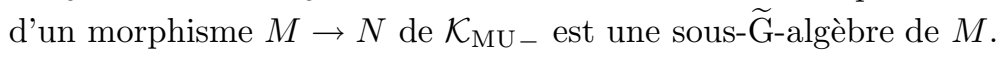

Proposition-DÉfinition 3.1.1. - Soit L un objet de $\widehat{\mathcal{L}}$.

(a) Le foncteur $\mathrm{h} \widehat{\mathcal{S}}_{\mathrm{pt}} \rightarrow \mathcal{E}$ ns-gr, $X \mapsto \operatorname{Hom}_{\widehat{\mathcal{M}}}\left(L, \widetilde{\widehat{\mathrm{MU}}^{*}} X\right)$ est représentable par un espace profini pointé fibrant sans p-torsion qu'on note $\mathrm{K}^{a}(L)$.

(b) Le morphisme d'algèbres instables non unitaires $\widetilde{\mathrm{G}}^{a}(L) \rightarrow \widetilde{\widehat{\mathrm{MU}}^{*}} \mathrm{~K}^{a}(L)$, adjoint de l'unité d'adjonction $L \rightarrow \widehat{\widehat{\mathrm{MU}}^{*}} \mathrm{~K}^{a}(L)$, est un isomorphisme naturel compatible avec les structures de monade de $\widetilde{\mathrm{G}}^{a}$ et de $L \mapsto \widetilde{\widehat{\mathrm{MU}}^{*}} \mathrm{~K}^{a}(L)$.

Démonstration. - Choisissons un ensemble gradué $S$ et un isomorphisme $\widehat{\mathrm{L}}(S) \rightarrow L$. Le foncteur $X \mapsto \operatorname{Hom}_{\widehat{M}}\left(\widehat{\mathrm{L}}(S), \widetilde{\widehat{\mathrm{MU}}^{*}} X\right)$ est représenté par l'espace profini pointé fibrant $\mathrm{K}(S)$ et le morphisme $\widehat{\mathrm{L}}(S) \rightarrow \widehat{\widehat{\mathrm{MU}}}^{*} \mathrm{~K}(S)$ adjoint dans $\widehat{\mathcal{M}}$ de l'unité $S \rightarrow \widehat{\widehat{\mathrm{MU}}}^{*} \mathrm{~K}(S)$. L'espace profini pointé $\mathrm{K}(S)$ représente également le foncteur $X \mapsto \operatorname{Hom}_{\widehat{\mathcal{M}}}\left(L, \widehat{\widehat{\mathrm{MU}}}^{*} X\right)$. Notons que la fonctorialité en $L$ du représentant dépend d'un choix. Le point (b) est formel.

Voici une caractérisation des espaces profinis pointés $\mathrm{K}^{a}(L), L \in \widehat{\mathcal{L}}$, (ou, de façon équivalente, de $\mathrm{K}(S), S \in \mathcal{E}$ ns-gr) à isomorphisme près :

LEMme 3.1.2. - Soit $X$ un espace profini pointé sans p-torsion dont la MUcohomologie continue réduite est isomorphe dans $\mathcal{K}_{\mathrm{MU}}$ à $\widetilde{\mathrm{G}}^{a}(L)$ pour un objet $L$ de $\widehat{\mathcal{L}}$; alors $X$ est isomorphe à $\mathrm{K}^{a}(L)$ dans $\mathrm{h} \widehat{\mathcal{S}}_{\mathrm{pt}}$

Démonstration. - La propriété universelle définissant $\mathrm{K}^{a}(L)$ montre que l'isomorphisme $\widetilde{\mathrm{G}}^{a}(L) \simeq \widetilde{\widehat{\mathrm{MU}}^{*}} X$ est induit par une application $X \rightarrow \mathrm{K}^{a}(L)$. Comme $X$ et 
$\mathrm{K}^{a}(L)$ sont sans $p$-torsion, le morphisme induit en cohomologie modulo $p$ continue réduite est la réduction modulo $\mathrm{f}^{1} \mathrm{du}$ morphisme en MU-cohomologie continue réduite donc est un isomorphisme.

Le lemme a une version non pointée qui se démontre de la même façon.

REMARQUE. - La structure multiplicative du spectre MU induit une structure de monade sur le foncteur h $\mathcal{S}_{\mathrm{pt}} \rightarrow \mathrm{h} \mathcal{S}_{\mathrm{pt}}, X \mapsto \Omega^{\infty}(\mathrm{MU} \wedge X)$. Si $X$ est un ensemble simplicial pointé connexe dont la cohomologie à coefficients $p$-adiques est libre et de dimension finie en chaque degré alors l'espace $\Omega^{\infty}(\mathrm{MU} \wedge X)$ a la même propriété $(c f$. [DL, appendice]) et on dispose d'un isomorphisme $\left(\Omega^{\infty}(\mathrm{MU} \wedge X)\right)^{\widehat{p}} \rightarrow \mathrm{K}^{a}\left(\widetilde{\mathrm{MU}^{*}} \widehat{X}^{p}\right)$ dans $\mathrm{h} \widehat{\mathcal{S}}_{\text {pt }}$ induisant un isomorphisme $\widetilde{\mathrm{G}}\left(\widehat{\mathrm{MU}}^{*} X\right) \rightarrow \widetilde{\mathrm{MU}}^{*} \Omega^{\infty}(\mathrm{MU} \wedge X)$ compatible avec les structures de monades. La structure de MU-algèbre instable non unitaire sur la MU-cohomologie complétée en $p$ réduite de tels espaces coïncide donc à dualité près avec celle définie dans $[\mathbf{B C M}]$.

Filtration squelettale. - Soient $S$ un ensemble gradué et $n$ un entier. L'inclusion $\mathrm{Sk}_{n} \mathrm{~K}(S) \rightarrow \mathrm{K}(S)$ induit un morphisme de G-algèbres $\mathrm{G}(S) \rightarrow \widehat{\mathrm{MU}}^{*} \mathrm{Sk}_{n} \mathrm{~K}(S)$ (de Galgèbres augmentées puisque $\mathrm{K}(S)$ est pointé) qui se factorise par la $\widehat{\mathrm{L}}$-algèbre quotient $\mathrm{G}(S) / \mathrm{F}^{n+1}$ (voir la filtration squelettale d'une $\widehat{\mathrm{L}}$-algèbre en section 2.2).

Comme l'espace profini $\mathrm{K}(S)$ est sans $p$-torsion, son squelette $n$-ième l'est également et les $\widehat{\mathrm{L}}$-algèbres $\mathrm{G}(S), \mathrm{G}(S) / \mathrm{F}^{n+1}$ et $\mathrm{Sk}_{n} \mathrm{~K}(S)$ sont libres. L'application $\mathrm{H}^{k} \mathrm{~K}(S) \rightarrow$ $\mathrm{H}^{k} \mathrm{Sk}_{n} \mathrm{~K}(S)$ est bijective pour $k<n$ et injective pour $k=n$ (cf. la démonstration du point (a) du lemme 2.1.7). Le morphisme de $\widehat{\mathrm{L}}$-algèbres $\mathrm{G}(S) / \mathrm{F}^{n+1} \rightarrow \widehat{\mathrm{MU}}^{*} \mathrm{Sk}_{n} \mathrm{~K}(S)$ est donc un monomorphisme modulo $\mathrm{f}^{1}$ donc est un monomorphisme par le point (b) de la proposition 2.1.3.

On en déduit que $\mathrm{G}(S) / \mathrm{F}^{n+1}$ s'identifie au coégalisateur du 1-complexe

$$
\mathrm{G}(S) \times \widehat{\mathrm{MU}}^{*} \mathrm{Sk}_{n} \mathrm{~K}(S) \mathrm{G}(S) \rightleftarrows \mathrm{G}(S)
$$

donc hérite de $\mathrm{G}(S)$ d'une structure naturelle de G-algèbre ( $c f$. la discussion sur les objets quotients en fin de la section A.1 de l'appendice A).

Soit $M$ une MU-algèbre instable. Puisque le foncteur $\widehat{\mathcal{M}} \rightarrow \widehat{\mathcal{M}}, N \mapsto N / \mathrm{F}^{n+1}$ commute aux coégalisateurs de 1-complexes, la $\widehat{\mathrm{L}}$-algèbre quotient $M / \mathrm{F}^{n+1}$ est le coégalisateur du 1-complexe de G-algèbres $\mathrm{G}^{2}(M) / \mathrm{F}^{n+1} \leftrightarrows \mathrm{G}(M) / \mathrm{F}^{n+1}$ donc hérite d'une structure de G-algèbre compatible avec celle de $M$.

Soit $X$ un espace profini. Le morphisme de MU-algèbres instables $\widehat{\mathrm{MU}}^{*} X \rightarrow$ $\widehat{\mathrm{MU}}^{*} \mathrm{Sk}_{n} X$ se factorise par $\left(\widehat{\mathrm{MU}}^{*} X\right) / \mathrm{F}^{n+1}$. Si $X$ est sans $p$-torsion alors le $\mathbb{F}_{p^{-}}$ espace vectoriel gradué $\left(\widehat{\mathrm{MU}}^{*} X / \mathrm{F}^{n+1}\right) / \mathrm{f}^{1}$ s'identifie à $\mathrm{H}^{*} X$ en degré inférieur ou égal à $n$ et est nul en degré strictement supérieur à $n$. Le morphisme $\widehat{\mathrm{MU}}^{*} X / \mathrm{F}^{n} \rightarrow$ $\left(\widehat{\mathrm{MU}}^{*} \mathrm{Sk}_{n} X\right) / \mathrm{F}^{n}$ est donc un isomorphisme modulo $\mathrm{f}^{1}$, donc un isomorphisme dans $\widehat{\mathcal{L}}$ (corollaire 2.1.4). Comme les tours $\left(\widehat{\mathrm{MU}}^{*} \mathrm{Sk}_{n} X\right)_{n}$ et $\left(\left(\widehat{\mathrm{MU}}^{*} \mathrm{Sk}_{n} X\right) / \mathrm{F}^{n}\right)_{n}$ sont proisomorphes, on en déduit le 
LEMME 3.1.3. - Soit $X$ un espace profini sans p-torsion, alors les morphismes $\widehat{\mathrm{MU}}^{*} X \rightarrow \widehat{\mathrm{MU}}^{*} \mathrm{Sk}_{n} X$ induisent un pro-isomorphisme

$$
\left(\left(\widehat{\mathrm{MU}}^{*} X\right) / \mathrm{F}^{n}\right)_{n} \stackrel{\sim}{\longrightarrow}\left(\widehat{\mathrm{MU}}^{*} \mathrm{Sk}_{n} X\right)_{n}
$$

entre tours d'objets de $\mathcal{K}_{\mathrm{MU}}$.

MU-algèbres instables et produit tensoriel. - Soient $S$ et $S^{\prime}$ deux ensembles gradués et notons $S \sqcup S^{\prime}$ leur réunion disjointe. Par adjonction la G-algèbre libre sur $S \sqcup S^{\prime}$ est la somme dans $\mathcal{K}_{\mathrm{MU}}\left(\right.$ et $\left.\mathcal{K}_{\mathrm{MU}_{0}}\right)$ de $\mathrm{G}(S)$ et $\mathrm{G}\left(S^{\prime}\right)$. L'espace profini $\mathrm{K}\left(S \sqcup S^{\prime}\right)$ est par définition le produit $\mathrm{K}(S) \times \mathrm{K}\left(S^{\prime}\right)$. L'isomorphisme de $\widehat{\mathrm{L}}$-algèbres $\mathrm{G}(S) \widehat{\otimes} \mathrm{G}\left(S^{\prime}\right) \rightarrow$ $\mathrm{G}\left(S \sqcup S^{\prime}\right)$ donné par la formule de Künneth fait du produit tensoriel $\mathrm{G}(S) \widehat{\otimes} \mathrm{G}\left(S^{\prime}\right)$ une G-algèbre (libre) fonctoriellement en $\mathrm{G}(S)$ et $\mathrm{G}\left(S^{\prime}\right)$ dans $\mathcal{K}_{\mathrm{MU}}$. Soient maintenant $M$ et $N$ deux MU-algèbres instables; alors $M \widehat{\otimes} N$ est canoniquement le coégalisateur dans $\widehat{\mathcal{M}}$ du 1-complexe de G-algèbres $\mathrm{G}^{2}(M) \widehat{\otimes} \mathrm{G}^{2}(N) \leftrightarrows \mathrm{G}(M) \widehat{\otimes} \mathrm{G}(N)$ (cf. l'appendice B) de sorte que $M \widehat{\otimes} N$ hérite d'une structure de G-algèbre naturelle en $M$ et $N$.

Proposition 3.1.4. - Soient $M$ et $N$ deux MU-algèbres instables.

(a) Les isomorphismes canoniques $\widehat{\mathrm{MU}}^{*} \widehat{\otimes} M \rightarrow M$ et $M \widehat{\otimes} \widehat{\mathrm{MU}}^{*} \rightarrow M$ dans $\widehat{\mathcal{M}}$ sont des isomorphismes dans $\mathcal{K}_{\mathrm{MU}}$.

(b) Les morphismes $M=M \widehat{\otimes} \widehat{\mathrm{MU}}^{*} \rightarrow M \widehat{\otimes} N$ et $N=\widehat{\mathrm{MU}}^{*} \widehat{\otimes} N \rightarrow M \widehat{\otimes} N$, induits par les morphismes $\widehat{\mathrm{MU}}^{*} \rightarrow M$ et $\widehat{\mathrm{MU}}^{*} \rightarrow N$, font de $M \widehat{\otimes} N$ la somme de $M$ et $N$ dans $\mathcal{K}_{\mathrm{MU}}$.

Démonstration. - On se ramène au cas «tautologique » où $M$ et $N$ sont les Galgèbres libres sur des ensembles gradués $S$ et $S^{\prime}$ par commutation de la somme et du produit tensoriel (proposition B.1) aux coégalisateurs de 1-complexes.

De même pour $M$ et $N$ deux MU-algèbres instables non unitaires, le produit tensoriel $M \widehat{\otimes} N$ a une structure naturelle de MU-algèbre instable non unitaire qui s'interprète comme celle associée au noyau du morphisme canonique entre algèbres instables augmentées de la somme $M_{+} \widehat{\otimes} N_{+}$dans le produit $(M \times N)_{+}$dans $\mathcal{K}_{\mathrm{MU}_{0}}$.

\section{REMARQUES}

- Soit $M$ dans $\mathcal{K}_{\mathrm{MU}}$; la composée du morphisme $M \otimes \widehat{\mathrm{MU}}^{*} M \rightarrow M \widehat{\otimes} M$ avec la somme des morphismes identité $M \widehat{\otimes} M \rightarrow M$ munit $M$ d'une structure de $\widehat{\mathrm{MU}}^{*}$ algèbre commutative unitaire.

- Soient $X$ et $Y$ deux espaces profinis. Le morphisme de $\widehat{\mathrm{L}}$-algèbres

$$
\widehat{\mathrm{MU}}^{*} X \widehat{\otimes} \widehat{\mathrm{MU}}^{*} Y \longrightarrow \widehat{\mathrm{MU}}^{*} X \times Y,
$$

induit par la structure de $\mathrm{MU}^{*}$-algèbre de $\widehat{\mathrm{MU}}^{*} X$ et de $\widehat{\mathrm{MU}}^{*} Y$ lorsque $X$ et $Y$ sont sans $p$-torsion, est la somme dans $\mathcal{K}_{\mathrm{MU}}$ des morphismes $\widehat{\mathrm{MU}}^{*} X \rightarrow \widehat{\mathrm{MU}}^{*} X \times Y$ et $\widehat{\mathrm{MU}}^{*} Y \rightarrow \widehat{\mathrm{MU}}^{*} X \times Y$ induits par les projections $X \times Y \rightarrow X$ et $X \times Y \rightarrow Y$. Il 
induit, lorsque $X$ et $Y$ sont pointés, un morphisme $\widetilde{\mathrm{MU}^{*}} X \widehat{\widetilde{\otimes}} \widetilde{\mathrm{MU}}^{*} Y \rightarrow{\widetilde{\mathrm{MU}^{*}}}^{*} \wedge Y$ dans $\mathcal{K}_{\mathrm{MU}-}$ qui est un isomorphisme si $X$ et $Y$ sont sans $p$-torsion.

3.2. Comparaison avec les algèbres instables pour la cohomologie modulo $p$. - Soit $n$ un entier. Le foncteur qui associe à un espace $X$ le groupe $\mathrm{Z}^{n} X$ de ses $n$-cocycles à valeurs dans $\mathbb{F}_{p}$ est représentable par le $\mathbb{F}_{p}$-espace vectoriel fini simplicial $[k] \mapsto \mathrm{Z}^{n} \triangle[k]$ qu'on note $\mathrm{K}\left(\mathbb{F}_{p}, n\right)$ et la bijection $\operatorname{Hom}_{\mathcal{S}}\left(X, \mathrm{~K}\left(\mathbb{F}_{p}, n\right)\right) \cong \mathrm{Z}^{n} X$ induit un isomorphisme $\operatorname{Hom}_{\mathrm{h} \mathcal{S}}\left(X, \mathrm{~K}\left(\mathbb{F}_{p}, n\right)\right) \cong \mathrm{H}^{n} X$ (cf. [Ma1, §23, 24]). Par exactitude des colimites filtrantes, ce même $\mathbb{F}_{p}$-espace vectoriel fini simplicial représente le groupe des $n$-cocycles continus d'un espace profini dans $\widehat{\mathcal{S}}$ et le $n$-ième groupe de cohomologie modulo $p$ continue dans $\mathrm{h} \widehat{\mathcal{S}}([\mathbf{M o 2}])$.

Notons $\mathcal{E}$ la catégorie des $\mathbb{F}_{p}$-espaces vectoriels gradués. Pour $E$ dans $\mathcal{E}$ on note

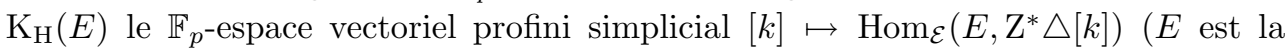
colimite filtrante de ses sous $\mathbb{F}_{p}$-espaces vectoriels de dimension totale finie). Ce qui précède montre qu'on a pour tout espace profini $X$ une bijection canonique $\operatorname{Hom}_{\widehat{\mathcal{S}}}\left(X, \mathrm{~K}_{\mathrm{H}}(E)\right) \cong \operatorname{Hom}_{\mathcal{E}}\left(E, \mathrm{Z}^{*} X\right)$ et en particulier un morphisme $E \rightarrow \mathrm{Z}^{*} \mathrm{~K}_{\mathrm{H}}(E)$.

Notons $\mathrm{F}^{0} E$ le sous $-\mathbb{F}_{p}$-espace vectoriel gradué de $E$ formé des éléments de degré positif ou nul. L'inclusion $\mathrm{F}^{0} E \rightarrow E$ induit un isomorphisme $\mathrm{K}_{\mathrm{H}}(E) \rightarrow \mathrm{K}_{\mathrm{H}}\left(\mathrm{F}^{0} E\right)$ puisqu'un espace profini n'a pas de cocycle non trivial en degré strictement négatif.

Lemme 3.2.1. - La composée $E \rightarrow \mathrm{Z}^{*} \mathrm{~K}_{\mathrm{H}}(E) \rightarrow \mathrm{H}^{*} \mathrm{~K}_{\mathrm{H}}(E)$ induit pour tout espace profini $X$ un isomorphisme $\operatorname{Hom}_{\mathrm{h} \widehat{\mathcal{S}}}\left(X, \mathrm{~K}_{\mathrm{H}}(E)\right) \cong \operatorname{Hom}_{\mathcal{E}}\left(E, \mathrm{H}^{*} X\right)$.

Démonstration. - On choisit un ensemble gradué $S$ et un isomorphisme du $\mathbb{F}_{p^{-} \text {-espace }}$ vectoriel gradué libre sur $S$ dans $E$. Cet isomorphisme induit un isomorphisme de $\mathrm{K}_{\mathrm{H}}(E)$ dans le produit $\prod_{s \in S} \mathrm{~K}\left(\mathbb{F}_{p},|s|\right)$ donc un isomorphisme de $\operatorname{Hom}_{\mathrm{h} \widehat{\mathcal{S}}}\left(X, \mathrm{~K}_{\mathrm{H}}(E)\right)$ dans le produit $\prod_{s \in S} \operatorname{Hom}_{\mathrm{h} \widehat{\mathcal{S}}}\left(X, \mathrm{~K}\left(\mathbb{F}_{p},|s|\right)\right)$, de sorte que le lemme se déduit du cas rappelé ci-dessus où $E$ est de dimension totale 1 .

L'espace profini $\mathrm{K}_{\mathrm{H}}(E)$ est pointé par l'adjoint dans $\widehat{\mathcal{S}}$ du morphisme nul $E \rightarrow \mathrm{Z}^{*}$ pt. Si $X$ est un espace profini pointé, le noyau $\widetilde{\mathrm{H}}^{*} X$ du morphisme $\mathrm{H}^{*} X \rightarrow \mathrm{H}^{*}$ pt s'identifie à l'ensemble $\operatorname{Hom}_{\mathrm{h} \widehat{\mathcal{S}}_{\mathrm{pt}}}\left(X, \mathrm{~K}_{\mathrm{H}}(E)\right)$.

On déduit du lemme et de sa version pointée des structures de monade sur les endofoncteurs $E \mapsto \mathrm{H}^{*} \mathrm{~K}_{\mathrm{H}}(E)$ et $E \mapsto \widetilde{\mathrm{H}}^{*} \mathrm{~K}_{\mathrm{H}}(E)$ de $\mathcal{E}$, qu'on note $\mathrm{G}_{\mathrm{H}}$ et $\widetilde{\mathrm{G}}_{\mathrm{H}}$ respectivement, et une structure de $\mathrm{G}_{\mathrm{H}}$-algèbre sur la cohomologie modulo $p$ continue d'un espace profini (respectivement de $\widetilde{\mathrm{G}}_{\mathrm{H}}$-algèbre sur la cohomologie modulo $p$ continue réduite d'un espace profini pointé). Observons que les foncteurs $G_{H}$ et $\widetilde{G}_{H}$ sont à valeurs dans la sous-catégorie de $\mathcal{E}$ formée des $\mathbb{F}_{p}$-espaces vectoriels gradués nuls en degré strictement négatif.

On note $\mathcal{K}_{\mathrm{H}}$ (respectivement $\mathcal{K}_{\mathrm{H}_{-}}$) la catégorie des $\mathrm{G}_{\mathrm{H}}$-algèbres de $\mathcal{E}$ (respectivement des $\widetilde{\mathrm{G}}_{\mathrm{H}}$-algèbres de $\mathcal{E}$ ) qu'on appelle $\mathrm{HZ} / p$-algèbres instables (respectivement $\mathrm{HZ} / p$-algèbres instables non unitaires). Observons que le coégalisateur dans $\mathcal{E}$ d'un 
1-complexe de $\mathrm{G}_{\mathrm{H}}$-algèbres ou de $\widetilde{\mathrm{G}}_{\mathrm{H}}$-algèbres est un coégalisateur scindé; autrement dit les monades $\mathrm{G}_{\mathrm{H}}$ et $\widetilde{\mathrm{G}}_{\mathrm{H}}$ vérifient la condition $\left(\mathcal{Q}_{0}\right)$ ( $c f$. appendice A). Le foncteur $\mathrm{G}_{\mathrm{H}}(\mathcal{E}) \rightarrow \mathcal{K}_{\mathrm{H}_{-}}, \mathrm{G}_{\mathrm{H}}(E) \mapsto \widetilde{\mathrm{H}}^{*}\left(\mathrm{~K}_{\mathrm{H}}(E)_{+}\right)$s'étend en un foncteur oubli $\mathcal{K}_{\mathrm{H}} \rightarrow \mathcal{K}_{\mathrm{H}_{-}}$ au dessus de $\mathcal{E}$, lequel admet un adjoint à gauche par le corollaire A.2.6 qu'on note $M \mapsto M_{+}$.

Une $\mathrm{G}_{\mathrm{H}}$-algèbre est exactement une algèbre instable sur l'algèbre de Steenrod modulo $p$ et pour $X$ un espace profini, la structure classique d'algèbre instable sur la cohomologie modulo $p$ continue de $X$ coïncide avec la structure de $\mathrm{G}_{\mathrm{H}}$-algèbre induite par l'unité $X \rightarrow \mathrm{K}_{\mathrm{H}}\left(\mathrm{H}^{*} X\right)$ : le cas où la $\mathrm{G}_{\mathrm{H}^{-}}$algèbre (respectivement l'espace profini) est finie en chaque degré résulte de $[\mathbf{L a 2}]$; le cas général est conséquence du lemme suivant.

\section{Lemme 3.2.2. - L'oubli $\mathcal{K}_{\mathrm{H}} \rightarrow \mathcal{E}$ commute aux colimites filtrantes.}

Démonstration. - Puisque la monade $\mathrm{G}_{\mathrm{H}}$ vérifie la condition $\left(\mathcal{Q}_{0}\right)$ il suffit par la proposition A.1.6 de vérifier que l'endofoncteur $\mathrm{G}_{\mathrm{H}}$ de $\mathcal{E}$ commute aux colimites filtrantes. Or le foncteur $E \mapsto \mathrm{K}_{\mathrm{H}}(E)$ transforme les colimites filtrantes en limites filtrantes par adjonction dans $\widehat{\mathcal{S}}$ et la cohomologie modulo $p$ continue d'une limite filtrante d'espaces profinis est la colimite des cohomologies modulo $p$ continues (lemme 1.1.1).

Le produit tensoriel dans $\mathcal{E}$ de deux algèbres instables est naturellement muni d'une structure d'algèbre instable qui en fait la somme dans $\mathcal{K}_{\mathrm{H}}$ (par un argument analogue à la preuve de la proposition 3.1.4) et qui est telle que pour toute paire d'espaces profinis $X, Y$ l'isomorphisme de Künneth $\mathrm{H}^{*} X \otimes \mathrm{H}^{*} Y \rightarrow \mathrm{H}^{*} X \times Y$ est un isomorphisme dans $\mathcal{K}_{\mathrm{H}}$.

Revenons aux MU-algèbres instables. Pour tout ensemble gradué $S$, le morphisme de $\mathbb{F}_{p}$-espaces vectoriels gradués $\mathrm{G}(S) / \mathrm{f}^{1} \rightarrow \mathrm{H}^{*} \mathrm{~K}(S)$ induit par le morphisme de spectres $\mathrm{MU} \rightarrow \mathrm{HZ} / p$ est un isomorphisme puisque $\mathrm{K}(S)$ est sans $p$-torsion et fait ainsi de $\mathrm{G}(S) / \mathrm{f}^{1}$ une $\mathrm{H} \mathbb{Z} / p$-algèbre instable fonctoriellement en $\mathrm{G}(S) \in \mathcal{K}_{\mathrm{MU}}$. Soit maintenant $M$ une MU-algèbre instable quelconque; le quotient $M / \mathrm{f}^{1}$ est canoniquement le coégalisateur dans $\mathcal{E}$ du 1-complexe $\mathrm{G}^{2}(M) / \mathrm{f}^{1} \leftrightarrows \mathrm{G}(M) / \mathrm{f}^{1}$ de $\mathcal{K}_{\mathrm{H}}$ donc hérite d'une structure de $\mathrm{HZ} / p$-algèbre instable fonctoriellement en $M \in \mathcal{K}_{\mathrm{MU}}$ et de façon compatible avec le morphisme naturel $\left(\widehat{\mathrm{MU}}^{*} X\right) / \mathrm{f}^{1} \rightarrow \mathrm{H}^{*} X$ pour tout espace profini $X$.

Les formules de Künneth en cohomologie modulo $p$ et en MU-cohomologie continues donnent pour toutes paires d'ensembles gradués $S, S^{\prime}$ un isomorphisme

$$
\left(\mathrm{G}(S) \widehat{\otimes} \mathrm{G}\left(S^{\prime}\right)\right) / \mathrm{f}^{1} \cong \mathrm{G}(S) / \mathrm{f}^{1} \otimes \mathrm{G}\left(S^{\prime}\right) / \mathrm{f}^{1} .
$$

On en déduit pour toute paire de MU-algèbres instables $M, M^{\prime}$ un isomorphisme $\left(M \widehat{\otimes} M^{\prime}\right) / \mathrm{f}^{1} \cong M / \mathrm{f}^{1} \otimes M^{\prime} / \mathrm{f}^{1}$ dans $\mathcal{K}_{\mathrm{H}}$ (observons que $M / \mathrm{f}^{1}$ et $M^{\prime} / \mathrm{f}^{1}$ sont nuls en degré strictement négatif) compatible avec les formules de Künneth pour la cohomologie continue d'un produit d'espaces profinis. 
La même discussion s'applique à la situation pointée.

Lemme 3.2.3. - Le foncteur $\mathcal{K}_{\mathrm{MU}} \rightarrow \mathcal{K}_{\mathrm{H}}, M \mapsto M / \mathrm{f}^{1}$ commute aux colimites indexées par une catégorie petite.

Démonstration. - Il suffit par la proposition A.1.6 de vérifier que la composée $\mathcal{E}$ ns-gr $\rightarrow \mathcal{K}_{\mathrm{H}}, S \mapsto \mathrm{G}(S) / \mathrm{f}^{1}$ commute aux sommes indexées par un ensemble. On observe d'abord que le foncteur $S \mapsto \mathrm{G}(S) / \mathrm{f}^{1} \cong \mathrm{H}^{*} \mathrm{~K}(S)$ commute aux colimites filtrantes par construction de $\mathrm{K}(S)$ dans $\widehat{\mathcal{S}}$ et par le lemme 1.1.1. Pour $S, S^{\prime}$ deux ensembles gradués l'isomorphisme $\mathrm{G}\left(S \sqcup S^{\prime}\right) / \mathrm{f}^{1} \cong \mathrm{G}(S) / \mathrm{f}^{1} \otimes \mathrm{G}\left(S^{\prime}\right) / \mathrm{f}^{1}$ et la discussion sur le produit tensoriel de deux objets de $\mathcal{K}_{\mathrm{H}}$ font de $\mathrm{G}\left(S \sqcup S^{\prime}\right) / \mathrm{f}^{1}$ la somme de $\mathrm{G}(S) / \mathrm{f}^{1}$ et de $\mathrm{G}\left(S^{\prime}\right) / \mathrm{f}^{1}$ dans $\mathcal{K}_{\mathrm{H}}$, d'où le résultat.

\subsection{Algèbres instables et $\pi_{0}$}

Proposition 3.3.1. - Pour toute MU-algèbre instable $M$ l'application naturelle

$$
\operatorname{Hom}_{\mathcal{K}_{\mathrm{MU}}}\left(M, \widehat{\mathrm{MU}}^{*}\right) \longrightarrow \operatorname{Hom}_{\mathcal{K}_{\mathrm{H}}}\left(M / \mathrm{f}^{1}, \mathbb{Z} / p\right)
$$

est une bijection.

Démonstration. - On se ramène au cas où $M$ est la G-algèbre libre sur un ensemble gradué $S$. Les deux ensembles sont alors canoniquement en bijection avec l'ensemble $\operatorname{Hom}_{\mathrm{h} \hat{\mathcal{S}}}(\mathrm{pt}, \mathrm{K}(S))$.

Remarque. - Les deux ensembles $\operatorname{Hom}_{\mathcal{K}_{\mathrm{MU}}}\left(M, \widehat{\mathrm{MU}}^{*}\right)$ et $\operatorname{Hom}_{\mathcal{K}_{\mathrm{H}}}\left(M / \mathrm{f}^{1}, \mathbb{Z} / p\right)$ ont une structure profinie naturelle et la bijection de la proposition est un homéomorphisme.

Corollaire 3.3.2. - Soit $M$ une MU-algèbre instable; les conditions suivantes sont équivalentes :

(i) La MU-algèbre instable $M$ est non nulle.

(ii) La $\mathrm{HZ} / p$-algèbre instable $M / \mathrm{f}^{1}$ est non nulle.

Si elles sont satisfaites le morphisme $\widehat{\mathrm{MU}}^{*} \rightarrow M$ admet un rétract dans $\mathcal{K}_{\mathrm{MU}}$.

Démonstration. - Si l'algèbre instable $M / \mathrm{f}^{1}$ est nulle alors $M$ est nulle par le lemme 2.2.3. Sinon l'unique morphisme $\mathbb{Z} / p \rightarrow M / \mathrm{f}^{1}$ dans $\mathcal{K}_{\mathrm{H}}$ admet un rétract (voir par exemple [La1, 1.7 et 1.8]) donc également le morphisme $\widehat{\mathrm{MU}}^{*} \rightarrow M$ dans $\mathcal{K}_{\mathrm{MU}}$ et $M$ est non nulle.

Proposition 3.3.3. - Soit X un espace profini fibrant; alors l'application naturelle

$$
\pi_{0} X \simeq \operatorname{Hom}_{\mathrm{h} \hat{\mathcal{S}}}(\mathrm{pt}, X) \longrightarrow \operatorname{Hom}_{\mathcal{K}_{\mathrm{MU}}}\left(\widehat{\mathrm{MU}}^{*} X, \widehat{\mathrm{MU}}^{*}\right)
$$

est une bijection. 
Démonstration. - Puisque les applications $\operatorname{Hom}_{\mathrm{h} \widehat{\mathcal{S}}}(\mathrm{pt}, X) \rightarrow \operatorname{Hom}_{\mathcal{K}_{\mathrm{H}}}\left(\mathrm{H}^{*} X, \mathbb{Z} / p\right)$ et $\operatorname{Hom}_{\mathcal{K}_{\mathrm{MU}}}\left(\widehat{\mathrm{MU}}^{*} X, \widehat{\mathrm{MU}}^{*}\right) \rightarrow \operatorname{Hom}_{\mathcal{K}_{\mathrm{H}}}\left(\widehat{\mathrm{MU}}^{*} X / \mathrm{f}^{1}, \mathbb{Z} / p\right)$ sont des bijections (proposition 3.3.1 pour la seconde), il suffit de montrer que l'application $\widehat{\mathrm{MU}}^{0} X / \mathrm{f}^{1} \rightarrow \mathrm{H}^{0} X$ est un isomorphisme. On observe d'abord que l'application $X \rightarrow \pi_{0} X$ induit un isomorphisme $\mathrm{H}^{0} \pi_{0} X \rightarrow \mathrm{H}^{0} X$ (cf. la section 1.3) et que le morphisme $\widehat{\mathrm{MU}}^{*} \pi_{0} X / \mathrm{f}^{1} \rightarrow$ $\mathrm{H}^{*} \pi_{0} X$ est iso puisque $\pi_{0} X$ est sans $p$-torsion. Puisque les composées $\widehat{\mathrm{MU}}^{0} \pi_{0} X / \mathrm{f}^{1} \rightarrow$ $\mathrm{H}^{0} \pi_{0} X \rightarrow \mathrm{H}^{0} X$ et $\widehat{\mathrm{MU}}^{0} \pi_{0} X / \mathrm{f}^{1} \rightarrow \widehat{\mathrm{MU}}^{0} X / \mathrm{f}^{1} \rightarrow \mathrm{H}^{0} X$ sont égales, on en déduit que l'application $\widehat{\mathrm{MU}}^{0} X / \mathrm{f}^{1} \rightarrow \mathrm{H}^{0} X$ est surjective. Posons $X^{0}=\mathrm{K}\left(\widehat{\mathrm{MU}}^{*} X\right)$ et notons $C$ la cofibre de l'application canonique $X \rightarrow X^{0}$. Comme $\widehat{\mathrm{MU}}^{*} X^{0} \rightarrow \widehat{\mathrm{MU}}^{*} X$ est épi, on dispose d'une suite exacte courte $0 \rightarrow{\widetilde{\mathrm{MU}^{*}}}^{*} \rightarrow \widehat{\mathrm{MU}}^{*} X^{0} \rightarrow \widehat{\mathrm{MU}}^{*} X \rightarrow 0$. On forme alors le diagramme

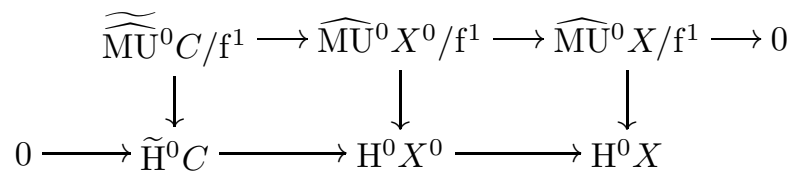

où les lignes sont exactes. Comme $X^{0}$ est sans $p$-torsion, la flèche verticale du milieu est iso. Comme les deux autres flèches verticales sont épi, celle de droite est iso, ce qui termine la preuve.

REMARQUE. - Les deux ensembles $\pi_{0} X$ (pour $X$ fibrant) et $\operatorname{Hom}_{\mathcal{K}_{\mathrm{MU}}}\left(\widehat{\mathrm{MU}}^{*} X, \widehat{\mathrm{MU}}^{*}\right)$ ont une structure profinie naturelle, le premier par construction ( $c f$. la section 1.3) et le second parce que $\widehat{\mathrm{MU}}^{*} X$ est la colimite filtrante de ses sous-MU-algèbres instables de type fini. L'application $\pi_{0} X \rightarrow \operatorname{Hom}_{\mathcal{K}_{\mathrm{MU}}}\left(\widehat{\mathrm{MU}}^{*} X, \widehat{\mathrm{MU}}^{*}\right)$ est continue donc est un homéomorphisme.

\section{Résolutions}

La section A.4 de l'appendice donne une courte présentation de la notion de résolution. Nous utilisons les résolutions libres pour étudier les propriétés d'exactitude à gauche de foncteurs additifs définis sur $\widehat{\mathcal{M}}$, tel que $M \mapsto M / \mathrm{f}^{1}$ ou $M \mapsto M \widehat{\otimes} N$, et pour étendre au cas général les résultats sur les $\widehat{L}$-algèbres libres ou sur les espaces profinis sans $p$-torsion établis dans la section 2.1 (filtration de la limite d'une tour de $\widehat{L}$-algèbres, formule de Künneth en MU-cohomologie continue, formule de coefficients universels reliant la MU-cohomologie continue à la cohomologie modulo $p$ continue).

La section 4.1 est consacrée aux résolutions libres des $\widehat{L}$-algèbres. Nous commençons par donner le lien entre l'annulation d'un dérivé gauche itéré du foncteur $(-) / \mathrm{f}^{1}$ en une $\widehat{\text { L-algèbre }} M$ et la longueur d'une résolution libre de $M$ (proposition 4.1.8). En particulier nous montrons qu'étant donnée une résolution libre $M_{*} \rightarrow M$ de $M$, la $\widehat{\mathrm{L}}$-algèbre $M$ est libre si et seulement si le complexe augmenté de $\mathbb{F}_{p}$-espaces vectoriels gradués $M_{*} / \mathrm{f}^{1} \rightarrow M / \mathrm{f}^{1}$ est acyclique (proposition 4.1 .3 ). 
Nous rappelons de l'appendice B la définition, pour $M, N$ deux $\widehat{L}$-algèbres, des dérivés gauches en $M$ du produit tensoriel par $N$ comme l'homologie du produit tensoriel par $N$ d'une résolution libre de $M$; on note cette homologie $\operatorname{Tor}_{*}(M, N)$. Le produit tensoriel par $N$ est exact si et seulement si la $\widehat{\mathrm{L}}$-algèbre $\operatorname{Tor}_{1}(M, N)$ est nulle pour toute $\widehat{\mathrm{L}}$-algèbre $M$. La difficulté rencontrée est que nous n'obtenons pas l'annulation de l'objet $\operatorname{Tor}_{1}(M, L)$ pour toute $\widehat{\mathrm{L}}$-algèbre libre $L$. Nous montrons que ces objets Tor coïncident avec les dérivés gauches du produit tensoriel dans la catégorie des $\widehat{\mathrm{MU}}^{*}$-modules sous certaines hypothèses de finitude (proposition 4.1.9).

Nous mettons ensuite en place la notion d'extension des scalaires associée à la sous $\mathbb{Z}_{p}$-algèbre $\Lambda_{n}$ de $\widehat{\mathrm{MU}}^{*}$ engendrée par les $n$ premiers générateurs de $\widehat{\mathrm{MU}}^{*}$ (voir $[\mathbf{C S}$ ] et [Ad1] pour le cas des $\widehat{\mathrm{MU}}^{*}$-modules) : A $\Lambda_{n}$ correspond une monade $\widehat{\mathrm{L}}_{n}$ sur $\mathcal{E}$ ns-gr, la catégorie abélienne $\widehat{\mathcal{M}}_{n}$ des $\widehat{\mathrm{L}}_{n}$-algèbres, un produit tensoriel $\widehat{\otimes}_{n}$ sur $\widehat{\mathcal{M}}_{n}$ et ses dérivés gauches $\operatorname{Tor}_{k}^{\widehat{\mathrm{L}}_{n}}$. Pour $n<n^{\prime} \in \mathbb{N} \cup\{\infty\}$ l'inclusion $\Lambda_{n} \subset \Lambda_{n^{\prime}}$ induit un foncteur oubli $\mathrm{O}_{n}: \widehat{\mathcal{M}}_{n^{\prime}} \rightarrow \widehat{\mathcal{M}}_{n}$, adjoint à droite d'un foncteur d'extension des scalaires $\mathrm{M}_{n}^{n^{\prime}}$ avec les propriétés suivantes :

- $\mathrm{O}_{n}$ et $\mathrm{M}_{n}^{n^{\prime}}$ transforment les objets libres en objets libres et sont exacts.

- On a un isomorphisme $\mathrm{O}_{n} \operatorname{Tor}_{*}^{\widehat{\mathrm{L}}_{n^{\prime}}}\left(M^{\prime}, \mathrm{M}_{n}^{n^{\prime}}(M)\right) \cong \operatorname{Tor}_{*}^{\widehat{\mathrm{L}}_{n}}\left(\mathrm{O}_{n} M^{\prime}, M\right)$ sous des hypothèses de finitude sur $M$ et $M^{\prime}$ (proposition 4.1.23).

Comme les $\widehat{\mathrm{L}}_{0}$-algèbres admettent une résolution libre de longueur 1 on en déduit :

- Pour $M$ une $\widehat{\mathrm{L}}$-algèbre et $L$ dans $\widehat{\mathcal{L}}$ l'objet $\operatorname{Tor}_{1}(M, L)$ est nul si $M$ est nul en degré assez grand ou si $L / \mathrm{f}^{1}$ est fini en chaque degré.

- Les $\widehat{\mathrm{L}}_{n}$-algèbres admettent une résolution libre de longueur finie.

En particulier si $M_{1} \rightarrow M_{0}$ est un morphisme dans $\widehat{\mathcal{L}}$ avec $M_{1} / \mathrm{f}^{1}$ et $M_{0} / \mathrm{f}^{1}$ concentrés en un nombre fini de degrés alors le conoyau dans $\widehat{\mathcal{M}}$ de $M_{1} \rightarrow M_{0}$ est dans l'image de $\mathrm{M}_{n}^{\infty}$ pour un certain entier $n$ donc admet une résolution libre de longueur finie (proposition 4.1.27).

Nous terminons la section par la comparaison des objets $\operatorname{Tor}_{*}\left(M, \lim _{s} N_{s}\right)$ et $\lim _{s} \operatorname{Tor}_{*}\left(M, N_{s}\right)$ pour $M$ une $\widehat{\mathrm{L}}$-algèbre et $\left(N_{s}\right)$ une tour de $\widehat{\mathrm{L}}$-algèbres sous des hypothèses de finitude garantissant un isomorphisme $M \widehat{\otimes} \lim _{s} N_{s} \cong \lim _{s}\left(M \widehat{\otimes} N_{s}\right)$ lorsque $\left(N_{s}\right)$ est une tour d'objets de $\widehat{\mathcal{L}}$ (proposition 4.1.31), en suivant les pas de la section 9 de $[\mathbf{R W Y}]$. Comme application nous prenons pour $M$ la $\widehat{\mathrm{L}}$-algèbre $\widehat{\mathrm{MU}}^{*} / \mathrm{f}^{n}$ et en déduisons le lien entre la filtration de la limite d'une tour de $\widehat{\mathrm{L}}$-algèbres et la limite des filtrations.

La section 4.2 s'intéresse aux résolutions instables et aux résolutions des espaces.

La structure de monade du foncteur $\mathrm{G}: S \mapsto \widehat{\mathrm{MU}} * \mathrm{~K}(S)$ permet d'associer à toute MU-algèbre instable $M$ une $G$-algèbre libre simpliciale $\mathrm{G}_{\bullet}(M)$, image en MUcohomologie continue d'un diagramme cosimplicial $\mathrm{K}^{\bullet}(M)$ d'espaces profinis de la forme $\mathrm{K}(S)$, et un morphisme $\mathrm{G}_{\bullet}(M) \rightarrow M$ qui est une résolution libre de $M$ 
dans $\widehat{\mathcal{M}}$. Si $X$ est un espace profini, on dispose d'un morphisme de l'objet cosimplicial constant $X$ dans $\mathrm{K}^{\bullet}\left(\widehat{\mathrm{MU}}^{*} X\right)$, lequel induit en MU-cohomologie continue la G-résolution simpliciale canonique de l'algèbre instable $\widehat{\mathrm{MU}}^{*} X$. La propriété importante est que si une algèbre instable $M$ est libre comme $\widehat{\mathrm{L}}$-algèbre alors le morphisme G. $(M) / \mathrm{f}^{1} \rightarrow M / \mathrm{f}^{1}$ entre algèbres instables simpliciales pour la cohomologie modulo $p$ est une résolution de $M / \mathrm{f}^{1}$.

Nous définissons la notion de MU-résolution libre d'un espace profini pointé $X$ comme la donnée d'une suite de cofibrations $\left(C^{n-1} \rightarrow X^{n}\right)_{n \geqslant 0}$ entre espaces profinis pointés vérifiant :

- On a $C^{-1}=X$ et $C^{n}=X^{n} / C^{n-1}$ pour tout $n \geqslant 0$.

- Pour tout entier $n \geqslant 0$ l'espace profini $X^{n}$ est sans $p$-torsion.

- Pour tout entier $n \geqslant 0$ l'application $C^{n-1} \rightarrow X^{n}$ induit un épimorphisme en MU-cohomologie continue réduite.

L'image en MU-cohomologie continue réduite du cocomplexe d'espaces profinis pointés $X \rightarrow X^{*}$ est alors une résolution libre de la MU-cohomologie continue réduite de $X$. On peut choisir chaque $X^{n}$ comme l'image par K d'un ensemble gradué $S$, auquel cas la résolution $X \rightarrow X^{*}$ est universelle parmi les MU-résolutions libres de $X$ (proposition 4.2.5). Si $X$ est de dimension finie alors $X$ admet une MU-résolution libre de longueur finie $X \rightarrow X^{*}$ avec chaque $X^{n}$ de dimension finie.

Dans la section 4.3 nous associons à une MU-résolution libre $X \rightarrow X^{*}$ d'un espace profini pointé $X$ et à une théorie cohomologique réduite $M^{*}(-)$ une suite spectrale dont le terme $\mathrm{E}_{2}^{-s, t}$ est l'homologie en degré $s$ du complexe $M^{t} X^{*}$. Cette suite spectrale converge fortement si la résolution de $X$ est de longueur finie. Nous obtenons ainsi sous certaines hypothèses de finitude la suite spectrale de Künneth de terme $\mathrm{E}_{2}^{-s, *}=\operatorname{Tor}_{s}\left(\widehat{\mathrm{MU}}^{*} X, \widehat{\mathrm{MU}}^{*} Y\right)$ convergeant fortement vers la MU-cohomologie continue du produit $X \times Y$ (proposition 4.3.1) et la suite spectrale de coefficients universels de terme $\mathrm{E}_{2}^{-s, *}=\operatorname{Tor}_{s}\left(\widehat{\mathrm{MU}}^{*} X, \widehat{\mathrm{MU}}^{*} / \mathrm{f}^{1}\right)$ convergeant fortement vers la cohomologie modulo $p$ continue de $X$ (proposition 4.3.3). ( $C f$. [Ad2] et $[\mathbf{C S}]$ pour le cas classique.)

\subsection{Résolutions libres dans $\widehat{\mathcal{M}}$ et torsion}

Résolutions libres. - Soit $M_{*}$ un complexe (nul en degré négatif) de $\widehat{\mathcal{M}}$; son homologie est un objet gradué de $\widehat{\mathcal{M}}$. Un complexe augmenté $M_{*} \rightarrow M$ est dit acyclique si le morphisme de complexes $M_{*} \rightarrow M$ induit un isomorphisme en homologie.

La proposition suivante est une traduction de la proposition A.4.1, où on prend pour $\mathcal{C}$ la catégorie opposée à $\widehat{\mathcal{M}}$ et pour $\mathcal{L}$ les objets de $\widehat{\mathcal{L}}$.

Proposition 4.1.1. - Soient $M_{*} \rightarrow M$ et $N_{*} \rightarrow N$ deux complexes augmentés de $\widehat{\mathcal{M}}$. On suppose que les objets $M_{n}$ sont dans $\widehat{\mathcal{L}}$ pour tout $n$ et que le complexe augmenté $N_{*} \rightarrow N$ est acyclique; alors tout morphisme de $M$ dans $N$ se relève en un morphisme de $M_{*}$ dans $N_{*}$ unique à homotopie près. 
On appelle résolution libre d'une $\widehat{\mathrm{L}}$-algèbre $M$ la donnée d'un complexe $M_{*}$ de $\widehat{\mathcal{L}}$ et d'une augmentation $M_{0} \rightarrow M$ tels que le complexe augmenté $M_{*} \rightarrow M$ soit acyclique. On appelle longueur d'une telle résolution la borne inférieure (éventuellement infinie) des entiers $n$ tels que $M_{k}$ est l'objet nul pour tout $k>n$.

Toute $\widehat{\mathrm{L}}$-algèbre $M$ admet une résolution libre canonique : la structure de monade de $\widehat{\mathrm{L}}$ permet d'associer à $M$ l'objet simplicial $\widehat{\mathrm{L}} \cdot(M)$ de $\widehat{\mathcal{L}}$ et le morphisme de $\widehat{\mathrm{L}} \cdot(M)$ dans la $\widehat{\mathrm{L}}$-algèbre simpliciale constante égale à $M$ est une équivalence d'homotopie entre les ensembles gradués simpliciaux sous-jacents de sorte que le complexe augmenté associé $\widehat{\mathrm{L}}_{*}(M) \rightarrow M$ est acyclique. On peut aussi observer que le complexe normalisé de la $\widehat{\mathrm{L}}$-algèbre simpliciale $\widehat{\mathrm{L}} .(M)$ est une résolution de $M$ et un complexe de $\widehat{\mathcal{L}}$ car rétract de $\widehat{\mathrm{L}}_{*}(M)$. La proposition qui précède montre que deux résolutions libres d'une même $\widehat{\mathrm{L}}$-algèbre sont équivalentes homotopiquement.

Le lemme suivant est une généralisation de la proposition 2.1.3 :

LEMME 4.1.2. - Soit $M^{\prime} \rightarrow M \rightarrow M^{\prime \prime}$ une suite de morphismes de $\widehat{\mathcal{L}}$ telle que la suite induite $M^{\prime} / \mathrm{f}^{1} \rightarrow M / \mathrm{f}^{1} \rightarrow M^{\prime \prime} / \mathrm{f}^{1}$ est exacte dans $\mathcal{E}$, alors la suite $M^{\prime} \rightarrow M \rightarrow M^{\prime \prime}$ est exacte dans $\widehat{\mathcal{M}}$ et le noyau du morphisme $M^{\prime} \rightarrow M$ comme le conoyau du morphisme $M \rightarrow M^{\prime \prime}$ sont dans $\widehat{\mathcal{L}}$.

Démonstration. - Nous montrons d'abord que le conoyau du morphisme $M^{\prime} \rightarrow M$ est dans $\widehat{\mathcal{L}}$. Notons le $N$; il suffit par le lemme 2.1.2 de vérifier que $N$ est complet et que l'application $\mathrm{f}^{n} \mathrm{MU}^{*} / \mathrm{f}^{n+1} \mathrm{MU}^{*} \otimes N / \mathrm{f}^{1} \rightarrow N / \mathrm{f}^{n+1}$ est injective pour tout $n$. Le second point vient de ce que la composée

$$
\mathrm{f}^{n} \mathrm{MU}^{*} / \mathrm{f}^{n+1} \mathrm{MU}^{*} \otimes N / \mathrm{f}^{1} \longrightarrow \mathrm{f}^{n} \mathrm{MU}^{*} / \mathrm{f}^{n+1} \mathrm{MU}^{*} \otimes M^{\prime \prime} / \mathrm{f}^{1} \longrightarrow M^{\prime \prime} / \mathrm{f}^{n+1}
$$

est injective (par l'hypothèse et le fait que $M^{\prime \prime}$ est dans $\widehat{\mathcal{L}}$ ) et qu'elle coïncide avec la composée $\mathrm{f}^{n} \mathrm{MU}^{*} / \mathrm{f}^{n+1} \mathrm{MU}^{*} \otimes N / \mathrm{f}^{1} \rightarrow N / \mathrm{f}^{n+1} \rightarrow M^{\prime \prime} / \mathrm{f}^{n+1}$. Notons $N^{\prime}$ le complété de $N ; N^{\prime}$ est dans $\widehat{\mathcal{L}}$ et la suite $M^{\prime} \rightarrow M \rightarrow N^{\prime}$ induit une suite exacte $M^{\prime} / \mathrm{f}^{1} \rightarrow$ $M / \mathrm{f}^{1} \rightarrow N^{\prime} / \mathrm{f}^{1} \rightarrow 0$ d'où on déduit que l'application $M \rightarrow N^{\prime}$ est surjective et que $M^{\prime}$ se surjecte sur le noyau de $M \rightarrow N^{\prime}$ par la proposition 2.1.3, donc $N^{\prime}$ égale $N$.

Le morphisme $N \rightarrow M^{\prime \prime}$ induit une injection $N / \mathrm{f}^{1} \rightarrow M^{\prime \prime} / \mathrm{f}^{1}$ donc est injectif et son conoyau est dans $\widehat{\mathcal{L}}$ par la proposition 2.1.3. De même l'image du morphisme $M^{\prime} \rightarrow M$, qui s'identifie au noyau de la surjection $M \rightarrow N$, est dans $\widehat{\mathcal{L}}$, donc également le noyau du morphisme $M^{\prime} \rightarrow M$ ce qui achève la démonstration.

Remarque. - Voici une reformulation du lemme : Soit $M^{\prime} \rightarrow M$ un morphisme de

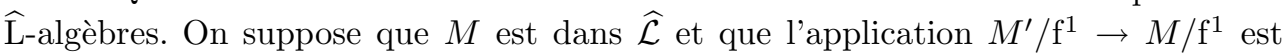
injective; alors $M^{\prime}$ est un rétract de $M$ (en particulier $M^{\prime}$ et le conoyau de $M^{\prime} \rightarrow M$ sont dans $\widehat{\mathcal{L}})$.

Rappelons que le foncteur $\widehat{\mathcal{M}} \rightarrow \mathcal{E}, M \mapsto M / \mathrm{f}^{1}$ commute aux conoyaux, de

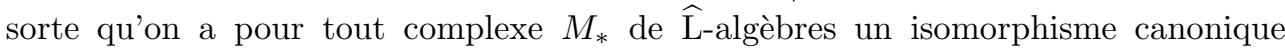
$\mathrm{H}_{0}\left(M_{*} / \mathrm{f}^{1}\right) \cong \mathrm{H}_{0}\left(M_{*}\right) / \mathrm{f}^{1}$. $\left(\mathrm{H}_{0}\left(M_{*}\right)\right.$ est le conoyau du morphisme $M_{1} \rightarrow M_{0}$. $)$ 
Proposition 4.1.3. - Soit $M_{*}$ un complexe de $\widehat{\mathrm{L}}$-algèbres tel que $M_{n}$ est dans $\widehat{\mathcal{L}}$ pour tout entier $n \geqslant 0$; alors :

(a) Les conditions suivantes sont équivalentes:

(i) Le complexe augmenté de $\mathbb{F}_{p}$-espaces vectoriels gradués

$$
M_{*} / \mathrm{f}^{1} \longrightarrow \mathrm{H}_{0}\left(M_{*} / \mathrm{f}^{1}\right)
$$

est acyclique.

(ii) La $\widehat{\mathrm{L}}$-algèbre $\mathrm{H}_{0}\left(M_{*}\right)$ est dans $\widehat{\mathcal{L}}$ et le complexe augmenté de $\widehat{\mathrm{L}}$-algèbres $M_{*} \rightarrow \mathrm{H}_{0}\left(M_{*}\right)$ est acyclique.

(b) Soient $M$ un objet de $\widehat{\mathcal{L}}$ et $\mathrm{H}_{0}\left(M_{*}\right) \rightarrow M$ un morphisme dans $\widehat{\mathcal{M}}$; alors $\mathrm{H}_{0}\left(M_{*}\right) \rightarrow M$ est un isomorphisme si et seulement si l'application induite $\mathrm{H}_{0}\left(M_{*} / \mathrm{f}^{1}\right) \rightarrow M / \mathrm{f}^{1}$ est un isomorphisme.

Démonstration. - Le point (b) est une conséquence directe du lemme 4.1.2, de même que l'implication (i) $\Rightarrow$ (ii) du point (a).

Supposons la condition (ii) du point (a) satisfaite et notons $K$ le noyau du morphisme $M_{0} \rightarrow \mathrm{H}_{0}\left(M_{*}\right)$. Comme $M_{0} \rightarrow \mathrm{H}_{0}\left(M_{*}\right)$ est épi, le morphisme $M_{0} / \mathrm{f}^{1} \rightarrow$ $\mathrm{H}_{0}\left(M_{*}\right) / \mathrm{f}^{1}$ est épi donc $K$ est un rétract de $M_{0}$ et est dans $\widehat{\mathcal{L}}$ par la proposition 2.1.3. En particulier la suite $0 \rightarrow K / \mathrm{f}^{1} \rightarrow M_{0} / \mathrm{f}^{1} \rightarrow \mathrm{H}_{0}\left(M_{*}\right) / \mathrm{f}^{1}$ est exacte. Le complexe augmenté $M_{*+1} \rightarrow K=\left(\cdots \rightarrow M_{1} \rightarrow K\right)$ vérifie encore la condition (ii) de sorte qu'on obtient inductivement la condition (i).

Voici une application de la proposition :

Lemme 4.1.4. - Soit $\left(L_{\alpha}\right)$ un diagramme filtrant d'objets de $\widehat{\mathcal{L}}$; alors la $\widehat{\mathrm{L}}$-algèbre colimite des $L_{\alpha}$ est dans $\widehat{\mathcal{L}}$.

Démonstration. - On regarde la $\widehat{\mathrm{L}}$-algèbre colimite des $L_{\alpha}$ comme le $\pi_{0}$ de la $\widehat{\mathrm{L}}$ algèbre simpliciale $\operatorname{colim}_{\alpha} \widehat{\mathrm{L}} \cdot\left(L_{\alpha}\right)$ induite par la résolution libre canonique des $L_{\alpha}$. Pour chaque entier $n \geqslant 1$ la $\widehat{\mathrm{L}}$-algèbre $\operatorname{colim}_{\alpha} \widehat{\mathrm{L}}^{n}\left(L_{\alpha}\right)$ est par adjonction la $\widehat{\mathrm{L}}$-algèbre libre sur la colimite des ensembles gradués sous-jacents aux $\widehat{\mathrm{L}}^{n-1}\left(L_{\alpha}\right)$. Il suffit donc de montrer que l'homologie du complexe $\left(\operatorname{colim}_{\alpha} \widehat{\mathrm{L}}_{*}\left(L_{\alpha}\right)\right) / \mathrm{f}^{1}$ est concentrée en degré 0 . Or cela vient de l'isomorphisme $\operatorname{colim}_{\alpha}\left(\widehat{\mathrm{L}}^{n}\left(L_{\alpha}\right) / \mathrm{f}^{1}\right) \rightarrow\left(\operatorname{colim}_{\alpha} \widehat{\mathrm{L}}_{*}\left(L_{\alpha}\right)\right) / \mathrm{f}^{1}$ (lemme 2.2.2) et de l'exactitude des colimites filtrantes dans $\mathcal{E}$.

Corollaire 4.1.5. - Soit $\left(X_{\alpha}\right)$ un diagramme filtrant d'espaces profinis sans $p$ torsion, alors les applications $\widehat{\mathrm{MU}}^{*} X_{\beta} \rightarrow \widehat{\mathrm{MU}}^{*} \lim _{\alpha} X_{\alpha}$ induisent un isomorphisme entre la $\widehat{\mathrm{L}}$-algèbre colimite des $\widehat{\mathrm{MU}}^{*} X_{\alpha}$ et $\widehat{\mathrm{MU}}^{*} \lim _{\alpha} X_{\alpha}$.

Démonstration. - On observe d'abord que l'espace profini $\lim _{\alpha} X_{\alpha}$ est sans $p$-torsion (lemme 2.1.15) donc que la $\widehat{\mathrm{L}}$-algèbre $\widehat{\mathrm{MU}}^{*} \lim _{\alpha} X_{\alpha}$ est dans $\widehat{\mathcal{L}}$. De même la $\widehat{\mathrm{L}}$ algèbre $\operatorname{colim}_{\alpha} \widehat{\mathrm{MU}}^{*} X_{\alpha}$ est dans $\widehat{\mathcal{L}}$ par le lemme 4.1.4. Il suffit donc de montrer que le morphisme $\operatorname{colim}_{\alpha} \widehat{\mathrm{MU}}^{*} X_{\alpha} \rightarrow \widehat{\mathrm{MU}}^{*} \lim _{\alpha} X_{\alpha}$ est un isomorphisme modulo $\mathrm{f}^{1}$ pour obtenir qu'il est un isomorphisme dans $\widehat{\mathcal{M}}$ (corollaire 2.1.4). Or ceci vient 
des isomorphismes $\left(\operatorname{colim}_{\alpha} \widehat{\mathrm{MU}}^{*} X_{\alpha}\right) / \mathrm{f}^{1} \simeq \operatorname{colim}_{\alpha} \mathrm{H}^{*} X_{\alpha}$ (par le lemme 2.2.2 et l'isomorphisme $\left.\left(\widehat{\mathrm{MU}}^{*} X_{\alpha}\right) / \mathrm{f}^{1} \simeq \mathrm{H}^{*} X_{\alpha}\right), \operatorname{colim}_{\alpha} \mathrm{H}^{*} X_{\alpha} \simeq \mathrm{H}^{*} \lim _{\alpha} X_{\alpha}$ (lemme 1.1.1) et $\mathrm{H}^{*} \lim _{\alpha} X_{\alpha} \simeq\left(\widehat{\mathrm{MU}}^{*} \lim _{\alpha} X_{\alpha}\right) / \mathrm{f}^{1}$ (car $\lim _{\alpha} X_{\alpha}$ est sans $p$-torsion $)$.

COROLlaire 4.1.6. - Le foncteur oubli $\mathcal{K}_{\mathrm{MU}} \rightarrow \widehat{\mathcal{M}}$ commute aux colimites filtrantes.

Démonstration. - Notons $\mathrm{O}^{a}$ le foncteur oubli $\mathcal{K}_{\mathrm{MU}} \rightarrow \widehat{\mathcal{M}}$. Il suffit d'après la proposition A.1.6 de vérifier que pour tout diagramme d'ensembles gradués $\left(S_{\alpha}\right)$ le morphisme $\mathrm{O}^{a} \mathrm{G}\left(\operatorname{colim}_{\alpha} S_{\alpha}\right) \rightarrow \operatorname{colim}_{\alpha} \mathrm{O}^{a} \mathrm{G}\left(S_{\alpha}\right)$ est un isomorphisme. Or ceci vient de ce que l'image par G d'un ensemble gradué $S$ est la MU-cohomologie continue de l'espace profini $\mathrm{K}(S)$, de ce qu'on a un isomorphisme $\mathrm{K}\left(\operatorname{colim}_{\alpha} S_{\alpha}\right) \simeq \lim _{\alpha} \mathrm{K}\left(S_{\alpha}\right)$ dans $\widehat{\mathcal{S}}$ par construction, et du corollaire ci-dessus.

Le lemme 4.1.2 implique également une version duale mais moins forte de la proposition 4.1 .3 :

Proposition 4.1.7. - Soit $M^{*}$ un cocomplexe de $\widehat{\mathcal{L}}$ tel que l'homologie du cocomplexe $M^{*} / \mathrm{f}^{1}$ est concentrée en degré 0 , alors l'homologie du cocomplexe $M^{*}$ est concentrée en degré 0 et le noyau du morphisme $M^{0} \rightarrow M^{1}$ est dans $\widehat{\mathcal{L}}$.

Exactitude du produit tensoriel et torsion. - Soient $M$ et $N$ deux $\widehat{\text { L-algèbres; on }}$ définit la $\widehat{\mathrm{L}}$-algèbre graduée $\operatorname{Tor}_{*}(M, N)$ comme l'homologie du complexe $\widehat{\mathrm{L}}_{*}(M) \widehat{\otimes} N$. L'invariance par homotopie implique que pour toute résolution libre $M_{*} \rightarrow M$ de $M$ il existe un isomorphisme canonique entre l'objet gradué $\operatorname{Tor}_{*}(M, N)$ et l'homologie du complexe $M_{*} \widehat{\otimes} N$. Par exactitude à droite du produit tensoriel, la $\widehat{\mathrm{L}}$-algèbre $\operatorname{Tor}_{0}(M, N)$ s'identifie à $M \widehat{\otimes} N$.

La suite de foncteurs $\left(\operatorname{Tor}_{k}(-,-)\right)$ a les propriétés suivantes $(c f$. appendice B) :

- Une suite exacte courte $0 \rightarrow M^{\prime} \rightarrow M \rightarrow M^{\prime \prime} \rightarrow 0$ de $\widehat{\text { L-algèbres induit pour }}$ toute $\widehat{\mathrm{L}}$-algèbre $N$ une suite exacte longue

$$
\cdots \longrightarrow \operatorname{Tor}_{1}(M, N) \longrightarrow \operatorname{Tor}_{1}\left(M^{\prime \prime}, N\right) \longrightarrow M^{\prime} \widehat{\otimes} N \longrightarrow M \widehat{\otimes} N \longrightarrow M^{\prime \prime} \widehat{\otimes} N \longrightarrow 0 .
$$

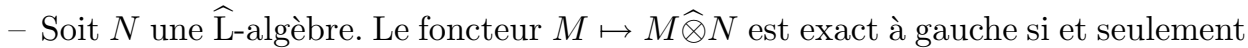
si la $\widehat{\text { L-algèbre }} \operatorname{Tor}_{1}(M, N)$ est nulle pour tout $M$.

- Soit $0 \rightarrow N^{\prime} \rightarrow N \rightarrow N^{\prime \prime} \rightarrow 0$ une suite exacte courte de $\widehat{\text { L-algèbres et supposons }}$ que $\operatorname{Tor}_{1}\left(N^{\prime \prime}, L\right)$ est nul pour tout $L \in \widehat{\mathcal{L}}$; alors la suite exacte courte induit pour tout $M$ une suite exacte

$$
\begin{aligned}
\operatorname{Tor}_{n+1}(M, N) \longrightarrow \operatorname{Tor}_{n+1}\left(M, N^{\prime \prime}\right) \longrightarrow \operatorname{Tor}_{n}\left(M, N^{\prime}\right) & \longrightarrow \cdots \\
& \longrightarrow M \widehat{\otimes} N \longrightarrow M \widehat{\otimes} N^{\prime \prime} \longrightarrow 0 .
\end{aligned}
$$

Nous montrerons plus loin (proposition 4.1.32) qu'on a pour toute $\widehat{\mathrm{L}}$-algèbre $M$ un isomorphisme naturel $M \widehat{\otimes} \widehat{M U}^{*} / \mathrm{f}^{1} \cong M / \mathrm{f}^{1}$. Si $M_{*} \rightarrow M$ est une résolution libre de $M$, le $n$-ième objet d'homologie du complexe $M_{*} / \mathrm{f}^{1} \mathrm{~s}$ 'interprète donc comme l'objet 
$\operatorname{Tor}_{n}\left(M, \widehat{\mathrm{MU}}^{*} / \mathrm{f}^{1}\right)$. Avec cette identification la proposition suivante est une généralisation de la proposition 4.1.3

Proposition 4.1.8. - Soit $M$ une $\widehat{\mathrm{L}}$-algèbre et $n$ un entier positif. Les conditions suivantes sont équivalentes :

(i) La $\widehat{\mathrm{L}}$-algèbre $\operatorname{Tor}_{n+1}\left(M, \mathrm{MU}^{*} / \mathrm{f}^{1}\right)$ est nulle.

(ii) Il existe une résolution libre de $M$ de longueur inférieure ou égale à $n$.

(iii) Pour toute résolution libre $M_{*} \rightarrow M$ de $M$, le conoyau du morphisme $M_{n+1} \rightarrow$ $M_{n}$ est dans $\widehat{\mathcal{L}}$.

Si elles sont vérifiées, les $\widehat{\mathrm{L}}$-algèbres $\operatorname{Tor}_{k}(M, N)$ sont nulles pour toute $\widehat{\mathrm{L}}$-algèbre $N$ et tout entier $k>n$.

Démonstration. - On utilise l'isomorphisme naturel $M \widehat{\otimes} \widehat{\mathrm{MU}}^{*} / \mathrm{f}^{1} \cong M / \mathrm{f}^{1}$ (proposition 4.1.32 (a)). La condition (i) implique (iii) par le lemme 4.1.2. La condition (iii) implique (ii) : la suite $\cdots \rightarrow 0 \rightarrow \operatorname{Coker}\left(M_{n+1} \rightarrow M_{n}\right) \rightarrow \cdots \rightarrow M$ est une résolution libre de $M$. Enfin (ii) implique clairement (i) et la dernière assertion de la proposition.

Exemple. - Nous verrons plus loin que la MU-cohomologie continue d'un espace profini de dimension finie admet une résolution libre de longueur finie.

Comparaison avec les modules $\operatorname{Tor}_{*}^{\widehat{\mathrm{MU}}^{*}}(M, N)$. — Soient $M$ et $N$ deux $\widehat{\mathrm{L}}$-algèbres, $M_{*} \rightarrow M$ une résolution libre de $M$ et $L_{*} \rightarrow M$ une résolution de $M$ par des $\widehat{\mathrm{MU}}^{*}$ modules libres. L'identité de $M$ se relève en un morphisme entre complexes de $\widehat{\mathrm{MU}}^{*}$ modules de $L_{*}$ dans $M_{*}$, unique à homotopie près. La composée $L_{*} \otimes_{\widehat{\mathrm{MU}}^{*}} N \rightarrow$ $M_{*} \otimes_{\widehat{\mathrm{MU}}^{*}} N \rightarrow M_{*} \widehat{\otimes} N$ induit un morphisme canonique $\operatorname{Tor}_{*}^{\widehat{\mathrm{MU}}^{*}}(M, N) \rightarrow \operatorname{Tor}_{*}(M, N)$ entre $\widehat{\mathrm{MU}}^{*}$-modules gradués.

Proposition 4.1.9. - Soient $M$ et $N$ deux $\widehat{\mathrm{L}}$-algèbres, $M_{*} \rightarrow M$ une résolution libre de $M$ et $N_{1} \rightarrow N_{0} \rightarrow N$ une présentation de $N$ dans $\widehat{\mathcal{L}}$. On suppose que l'une des conditions suivantes est satisfaite :

(1) Les $\mathbb{F}_{p}$-espaces vectoriels gradués $M_{n} / \mathrm{f}^{1}, n \in \mathbb{N}$ et $N_{n} / \mathrm{f}^{1}, n \in\{0,1\}$ sont nuls en degré assez grand et l'une des familles est formée d'espaces vectoriels gradués finis en chaque degré.

(2) L'une des familles $M_{n} / \mathrm{f}^{1}, n \in \mathbb{N}$ ou $N_{n} / \mathrm{f}^{1}, n \in\{0,1\}$ est formée d'espaces vectoriels gradués de dimension totale finie.

Alors pour tout entier $n$ le morphisme $\operatorname{Tor}_{n}^{\widehat{\mathrm{MU}}^{*}}(M, N) \rightarrow \operatorname{Tor}_{n}(M, N)$ est un isomorphisme de $\widehat{\mathrm{MU}}^{*}$-modules.

Démonstration. - Sous l'une des conditions (1) ou (2) le $\widehat{\mathrm{MU}}^{*}$-module gradué sousjacent à l'objet $\operatorname{Tor}_{*}(M, N)$ s'identifie à l'homologie du complexe $M_{*} \otimes_{\widehat{\mathrm{MU}}^{*}} N$. Il suffit donc de montrer que les $\widehat{\mathrm{MU}}^{*}$-modules $M_{n}$ sont plats pour tout $n$. Or cela vient de 
ce que $M_{n}$ est dans $\widehat{\mathcal{L}}$ et est nul en degré assez grand, donc est isomorphe au produit tensoriel au dessus de $\mathbb{Z}_{p}$ de $\widehat{\mathrm{MU}}^{*}$ et d'un $\mathbb{Z}_{p}$-module sans torsion (de façon non canonique).

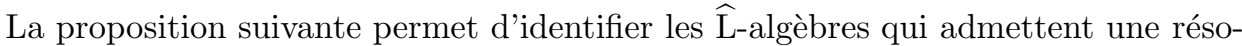
lution libre vérifiant la condition (1) ci-dessus. Rappelons que la catégorie $\widehat{\mathcal{M}}^{0}$ des $\widehat{L}$-algèbres nulles en degré assez grand s'identifie à une sous-catégorie pleine de $\mathcal{M}$ (proposition 2.2.1).

Proposition 4.1.10. - Soit $M$ un $\widehat{\mathrm{MU}}^{*}$-module nul en degré assez grand qu'on munit de la filtration par les coefficients; les conditions suivantes sont équivalentes :

(i) Le $\widehat{\mathrm{MU}}^{*}$-module $M$ admet un ensemble gradué de générateurs fini en chaque degré.

(ii) Le $\mathbb{Z}_{p}$-module gradué sous-jacent à $M$ est de type fini en chaque degré.

(iii) $M$ est une $\widehat{\mathrm{L}}$-algèbre et $M / \mathrm{f}^{1}$ est fini en chaque degré.

Si elles sont vérifiées, $M$ est complet pour la filtration par les coefficients.

Démonstration. - Supposons la condition (iii) satisfaite. Soit $S$ une base du $\mathbb{F}_{p^{-}}$ espace vectoriel gradué $M / \mathrm{f}^{1}$ qu'on relève en une famille d'éléments de $M$; l'application $S \rightarrow M$ induit un morphisme de $\widehat{\mathrm{L}}$-algèbres $\widehat{\mathrm{L}}(S) \rightarrow M$ dont on note $Q$ le conoyau. Comme l'espace vectoriel gradué $Q / \mathrm{f}^{1}$ est le conoyau du morphisme $\widehat{\mathrm{L}}(S) / \mathrm{f}^{1} \rightarrow M / \mathrm{f}^{1}$, il est nul. On en déduit que la $\widehat{\mathrm{L}}$-algèbre $Q$ est nulle en utilisant le début d'une résolution libre de $Q$ et la proposition 2.1.3. Autrement dit l'application $\widehat{\mathrm{L}}(S) \rightarrow M$ est surjective, ce qui implique la condition (i). La condition (i) implique la condition (ii), laquelle implique que $M$ est fini en chaque degré modulo $\mathrm{f}^{1}$ et qu'il est complet pour la filtration $p$-adique donc est dans $\widehat{\mathcal{M}}^{0}$ par la proposition 2.2.1. Observons également que la condition (i) se déduit facilement de (ii) et qu'elle implique que $M$ est complet pour la filtration par les coefficients : on le vérifie d'abord lorsque $M$ est un $\widehat{\mathrm{MU}}^{*}$-module libre et on l'obtient dans le cas général par l'absence de terme lim${ }^{1}$.

Corollaire 4.1.11. - Soit $M$ une $\widehat{\mathrm{L}}$-algèbre nulle en degré assez grand et finie en chaque degré modulo $\mathrm{f}^{1}$; alors $M$ admet une résolution par des objets de $\widehat{\mathcal{L}}$ vérifiant les mêmes propriétés.

Démonstration. - On construit inductivement une résolution libre $M_{*} \rightarrow M$ de $M$ avec les propriétés requises. Le fait que $M$ vérifie la condition (i) de la proposition montre l'existence de $M_{0}$. Le fait que $M_{0}$ et $M$ vérifient la condition (ii) de la proposition implique que le noyau du morphisme $M_{0} \rightarrow M$ vérifie la même condition donc vérifie les hypothèses faites sur $M$, ce qui permet de conclure.

Extension des scalaires et $\widehat{\mathrm{L}}$-algèbres de présentation en degré borné. - Rappelons que l'anneau $\widehat{\mathrm{MU}}^{*}$ est une $\mathbb{Z}_{p}$-algèbre polynomiale en des générateurs $x_{n}$ de degré $-2 n, n \geqslant 1$. On note $\Lambda_{0}$ l'anneau $\mathbb{Z}_{p}$ des entiers $p$-adiques et, pour $n \geqslant 1, \Lambda_{n}$ la 
sous- $\mathbb{Z}_{p}$-algèbre de $\widehat{\mathrm{MU}}^{*}$ engendrée par $x_{1}, \ldots, x_{n}$. La filtration de $\widehat{\mathrm{MU}}^{*}$ induit une filtration de $\Lambda_{n}$ pour laquelle $\Lambda_{n}$ est complet, et une filtration par les coefficients des $\Lambda_{n}$-modules.

On note $\widehat{\mathrm{L}}_{n}(S)$ le complété pour la filtration par les coefficients du $\Lambda_{n}$-module libre de base un ensemble gradué $S$, muni de la filtration limite, et $\widehat{\mathcal{L}}_{n}$ la catégorie des $\Lambda_{n}$-modules filtrés isomorphes à $\widehat{\mathrm{L}}_{n}(S)$ pour un certain $S \in \mathcal{E}$ ns-gr. Le foncteur $\widehat{\mathrm{L}}_{n}$ est adjoint à gauche de l'oubli de la catégorie des $\Lambda_{n}$-modules complets pour la filtration par les coefficients dans $\mathcal{E}$ ns-gr. Sa composée avec l'oubli, qu'on note encore $\widehat{\mathrm{L}}_{n}$, est donc une monade sur $\mathcal{E}$ ns-gr. On note $\mathcal{M}_{n}$ la catégorie des $\Lambda_{n}$-modules et $\widehat{\mathcal{M}}_{n}$ celle des $\widehat{\mathrm{L}}_{n}$-algèbres de $\mathcal{E}$ ns-gr. Comme pour $\widehat{\mathcal{M}}$, la catégorie $\widehat{\mathcal{M}}_{n}$ est abélienne. L'oubli $\widehat{\mathcal{M}}_{n} \rightarrow \mathcal{M}_{n}$ est additif, exact et admet un adjoint à gauche qu'on note $\widehat{\mathrm{L}}_{n}^{a}$; il se relève en une équivalence de catégories $\widehat{\mathcal{M}}_{n} \rightarrow \mathcal{M}_{n}\left(\widehat{\mathrm{L}}_{n}^{a}\right)$, où on a également noté $\widehat{\mathrm{L}}_{n}^{a}$ la monade composée de $\widehat{\mathrm{L}}_{n}^{a}$ avec l'oubli (proposition A.3.3).

LEMME 4.1.12. - Soit $S$ un ensemble gradué.

(a) $\widehat{\mathrm{L}}_{n}(S)$ s'identifie comme $\Lambda_{n}$-module au $\Lambda_{n}$-module formé en chaque degré $j$ des familles d'applications $\left(S^{i} \rightarrow \Lambda_{n}^{j-i}\right)_{i \in \mathbb{Z}}$ telles que pour tout $i$ et tout $k \geqslant 0$ l'application induite $S^{i} \rightarrow\left(\Lambda_{n} / \mathrm{f}^{k}\right)^{j-i}$ est à support fini.

(b) La filtration limite de $\widehat{\mathrm{L}}_{n}(S)$ est la filtration par les coefficients.

La proposition suivante est le pendant de la proposition 2.2.1 et est conséquence du lemme ci-dessus.

\section{Proposition 4.1.13}

(a) L'oubli $\widehat{\mathcal{M}}_{n} \rightarrow \mathcal{M}_{n}$ est pleinement fidèle d'image les $\widehat{\mathrm{L}}_{n}^{a}$-algèbres libres de $\mathcal{M}_{n}$.

(b) la filtration des objets de $\widehat{\mathcal{M}}_{n}$ est la filtration par les coefficients.

On démontre le lemme suivant de la même façon que le lemme 2.1.2

LEMME 4.1.14. - Soit $M$ un $\Lambda_{n}$-module muni d'une filtration plus grossière que la filtration par les coefficients; alors $M$ est isomorphe au $\Lambda_{n}$-module filtré $\widehat{\mathrm{L}}_{n}(S)$ pour un $S \in \mathcal{E}$ ns-gr si et seulement si les trois conditions suivantes sont satisfaites :

(1) $M$ est complet pour la filtration.

(2) Pour tout entier $k$ l'application $\Lambda_{n} / \mathrm{f}^{k} \otimes_{\Lambda_{n}} M / \mathrm{f}^{k+1} \rightarrow M / \mathrm{f}^{k}$ est un isomorphisme.

(iii) Pour tout entier $k$ la suite exacte $0 \rightarrow \mathrm{f}^{k} \Lambda_{n} / \mathrm{f}^{k+1} \Lambda_{n} \rightarrow \Lambda_{n} / \mathrm{f}^{k+1} \rightarrow \Lambda_{n} / \mathrm{f}^{k} \rightarrow 0$ induit une suite exacte

$$
0 \longrightarrow \mathrm{f}^{k} \Lambda_{n} / \mathrm{f}^{k+1} \Lambda_{n} \otimes_{\Lambda_{n}} M / \mathrm{f}^{1} \longrightarrow M / \mathrm{f}^{k+1} \longrightarrow M / \mathrm{f}^{k} \longrightarrow 0 .
$$

En particulier on a : 
LEMme 4.1.15. - Soit $M$ un $\mathbb{Z}_{p}$-module muni de la filtration par les coefficients, alors $M$ est dans $\widehat{\mathcal{L}}_{0}$ si et seulement si $M$ est complet pour la filtration $p$-adique et sans torsion.

On en déduit d'autre part, comme pour le lemme 2.1.12, le

Lemme 4.1.16. - Soit $\left(M_{s}\right)$ une tour d'objets de $\widehat{\mathcal{L}}_{n}$ telle que la tour de $\mathbb{F}_{p}$-espaces vectoriels gradués $\left(M_{s} / \mathrm{f}^{1}\right)$ est sans $\lim ^{1}$; alors la tour de $\Lambda_{n}$-modules $\left(M_{s}\right)$ est sans $\lim ^{1}$, le $\Lambda_{n}$-module $M_{\infty}=\lim _{s} M_{s}$ muni de la filtration limite est dans $\widehat{\mathcal{L}}_{n}$ et l'application $M_{\infty} / \mathrm{f}^{k} \rightarrow \lim _{s} M_{s} / \mathrm{f}^{k}$ est un isomorphisme pour tout entier $k$.

(Rappelons que la filtration des objets de $\widehat{\mathcal{M}}_{n}$ est la filtration par les coefficients par le premier lemme ci-dessus.)

On convient de poser $\Lambda_{\infty}=\widehat{\mathrm{MU}}^{*}$ et $\widehat{\mathrm{L}}_{\infty}=\widehat{\mathrm{L}}$. Pour $S$ dans $\mathcal{E}$ ns-gr et $k$ dans $\mathbb{Z}$ on note $\mathrm{F}_{n}^{k} \widehat{\mathrm{L}}_{n}(S)$ la sous $\widehat{\mathrm{L}}_{n}$-algèbre de $\widehat{\mathrm{L}}_{n}(S)$ engendrée par les éléments de degré supérieur ou égal à $k$. C'est un facteur direct de $\widehat{\mathrm{L}}_{n}(S)$ et $\widehat{\mathrm{L}}_{n}(S)$ est la limite de la tour de $\Lambda_{n}$-modules filtrés $\left(\widehat{\mathrm{L}}_{n}(S) / \mathrm{F}_{n}^{k} \widehat{\mathrm{L}}_{n}(S)\right)_{k}$

Lemme 4.1.17. - Soient $n<n^{\prime}$ deux éléments de $\mathbb{N} \cup\{\infty\}$ et $S$ un ensemble gradué; alors le $\Lambda_{n}$-module sous-jacent $\grave{a} \widehat{\mathrm{L}}_{n^{\prime}}(S)$, muni de la filtration par les coefficients, est dans $\widehat{\mathcal{L}}_{n}$.

Démonstration. - On observe d'abord que le $\Lambda_{n}$-module $\Lambda_{n^{\prime}}$ est isomorphe à l'image par $\widehat{\mathrm{L}}_{n}$ d'un ensemble gradué, disons $S^{\prime}$, fini en chaque degré et vide en degré assez grand. Si $S$ est un ensemble gradué vide en degré assez grand, les $\Lambda_{n}$-modules $\widehat{\mathrm{L}}_{n^{\prime}}(S)$ et $\widehat{\mathrm{L}}_{n}(S) \otimes_{\Lambda_{n}} \Lambda_{n^{\prime}}$ sont isomorphes. On en déduit facilement (à l'aide du lemme 4.1.14 par exemple) que le $\Lambda_{n}$-module $\widehat{\mathrm{L}}_{n^{\prime}}(S)$ est isomorphe à $\widehat{\mathrm{L}}_{n}\left(S \times S^{\prime}\right)$. Le cas général s'obtient en appliquant le lemme 4.1.16 (ou 2.1.12 si $n^{\prime}$ est infini) à la tour d'épimorphismes $\left(\widehat{\mathrm{L}}_{n^{\prime}}(S) / \mathrm{F}_{n^{\prime}}^{k}\right)_{k}$.

Soient $n<n^{\prime}$ deux éléments de $\mathbb{N} \cup\{\infty\}$. Pour tout ensemble gradué $S$, la $\widehat{\mathrm{L}}_{n^{\prime}}$ algèbre libre sur $S$ est dans $\widehat{\mathcal{L}}_{n}$ par le lemme ci-dessus. On en déduit par adjonction un morphisme $\widehat{\mathrm{L}}_{n}(S) \rightarrow \widehat{\mathrm{L}}_{n^{\prime}}(S)$ dans $\widehat{\mathcal{L}}_{n}$ compatible avec les structures de monades de $\widehat{\mathrm{L}}_{n}$ et $\widehat{\mathrm{L}}_{n^{\prime}}$. On en déduit une structure naturelle de $\widehat{\mathrm{L}}_{n}$-algèbre sur les $\widehat{\mathrm{L}}_{n^{\prime}}$-algèbres, c'est à dire un foncteur oubli $\mathrm{O}_{n}: \widehat{\mathcal{M}}_{n^{\prime}} \rightarrow \widehat{\mathcal{M}}_{n}$ au dessus de $\mathcal{E}$ ns-gr ( $c f$. le lemme A.2.5). Cet oubli est additif, exact et admet un adjoint à gauche qu'on note $\mathrm{M}_{n}^{n^{\prime}}$ par la proposition A.3.3. Le foncteur associé $\widehat{\mathcal{M}}_{n^{\prime}} \rightarrow \widehat{\mathcal{M}}_{n}\left(\mathrm{O}_{n} \mathrm{M}_{n}^{n^{\prime}}\right)$ est une équivalence de catégories par la même proposition. Par construction, l'image par $\mathrm{M}_{n}^{n^{\prime}}$ de la $\widehat{\mathrm{L}}_{n}$-algèbre libre sur $S$ est la $\widehat{\mathrm{L}}_{n^{\prime}}$-algèbre libre sur $S$ (cf. appendice A.2).

On définit un produit tensoriel $-\otimes_{\widehat{\mathrm{L}}_{n}}-\operatorname{sur} \widehat{\mathcal{M}}_{n}$ en posant

$\widehat{\mathrm{L}}_{n}(S) \otimes_{\widehat{\mathrm{L}}_{n}} \widehat{\mathrm{L}}_{n}\left(S^{\prime}\right)=\widehat{\mathrm{L}}_{n}\left(S \times S^{\prime}\right)$ pour $S$ et $S^{\prime}$ vides en degré assez grand,

$\widehat{\mathrm{L}}_{n}(S) \otimes_{\widehat{\mathrm{L}}_{n}} \widehat{\mathrm{L}}_{n}\left(S^{\prime}\right)=\lim _{k}\left(\widehat{\mathrm{L}}_{n}(S) / \mathrm{F}_{n}^{k} \otimes_{\widehat{\mathrm{L}}_{n}} \widehat{\mathrm{L}}_{n}\left(S^{\prime}\right) / \mathrm{F}_{n}^{k}\right)$ pour $S$ et $S^{\prime}$ quelconques, puis pour $M$ et $N$ deux $\widehat{\mathrm{L}}_{n}$-algèbres, $M \otimes_{\widehat{\mathrm{L}}_{n}} N$ est le coégalisateur du 1-complexe 
$\widehat{\mathrm{L}}_{n}^{2}(M) \otimes_{\widehat{\mathrm{L}}_{n}} \widehat{\mathrm{L}}_{n}^{2}(N) \rightrightarrows \widehat{\mathrm{L}}_{n}(M) \otimes_{\widehat{\mathrm{L}}_{n}} \widehat{\mathrm{L}}_{n}(N)$ (cf. l'appendice B). Alors le foncteur $N \mapsto$ $M \otimes_{\widehat{\mathrm{L}}_{n}} N$ est additif et exact à droite (proposition B.1). Il admet une suite de dérivés gauches $\left(N \mapsto \operatorname{Tor}_{k}^{\widehat{\mathrm{L}}_{n}}(M, N)\right)_{k}$ définie comme l'homologie du complexe $\left.\widehat{\mathrm{L}}_{n *}(M) \otimes_{\widehat{\mathrm{L}}_{n}} N\right)$ (appendice B).

Soient à nouveau $n<n^{\prime}$ deux éléments de $\mathbb{N} \cup\{\infty\}$. Pour $M$ et $N$ deux $\widehat{\mathrm{L}}_{n^{\prime}}$ algèbres libres, la structure d'anneau de $\Lambda_{n^{\prime}}$ donne un morphisme $\mathrm{O}_{n} M \otimes_{\widehat{\mathrm{L}}_{n}} \mathrm{O}_{n} N \rightarrow$ $\mathrm{O}_{n}\left(M \otimes_{\widehat{\mathrm{L}}_{n^{\prime}}} N\right)$. On en déduit pour $M$ et $N$ quelconque un morphisme naturel

$$
\mathrm{O}_{n} M \otimes_{\widehat{\mathrm{L}}_{n}} \mathrm{O}_{n} N \longrightarrow \mathrm{O}_{n}\left(M \otimes_{\widehat{\mathrm{L}}_{n^{\prime}}} N\right) .
$$

Soient maintenant $M$ une $\widehat{\mathrm{L}}_{n}$-algèbre et $N$ une $\widehat{\mathrm{L}}_{n^{\prime}}$-algèbre. L'unité d'adjonction $M \rightarrow$ $\mathrm{O}_{n} \mathrm{M}_{n}^{n^{\prime}}(M)$ et le morphisme précédemment décrit induisent un morphisme

$$
M \otimes_{\widehat{\mathrm{L}}_{n}} \mathrm{O}_{n} N \longrightarrow \mathrm{O}_{n}\left(\mathrm{M}_{n}^{n^{\prime}}(M) \otimes_{\widehat{\mathrm{L}}_{n^{\prime}}} N\right) .
$$

Lemme 4.1.18. - Soient $n<n^{\prime}$ deux éléments de $\mathbb{N} \cup\{\infty\}$ et soient $L$ et $L^{\prime}$ deux objets de $\widehat{\mathcal{L}}_{n}$ et $\widehat{\mathcal{L}}_{n^{\prime}}$ respectivement. Le morphisme $L \otimes_{\widehat{\mathrm{L}}_{n}} \mathrm{O}_{n} L^{\prime} \rightarrow \mathrm{O}_{n}\left(\mathrm{M}_{n}^{n^{\prime}}(L) \otimes_{\widehat{\mathrm{L}}_{n^{\prime}}} L^{\prime}\right)$ est un isomorphisme si et seulement si $L / \mathrm{f}^{1}$ est fini en chaque degré ou $L^{\prime}$ est nul en degré assez grand.

Démonstration. - Choisissons un isomorphisme $\widehat{\mathrm{L}}_{n}(S) \cong L$ pour un certain ensemble gradué $S$. Il suffit pour montrer le lemme d'observer que la $\widehat{\mathrm{L}}_{n^{\prime}}$-algèbre $\mathrm{M}_{n}^{n^{\prime}}(L)$ s'identifie à $\widehat{\mathrm{L}}_{n^{\prime}}(S)$, que $\mathrm{M}_{n}^{n^{\prime}}(L) \otimes_{\widehat{\mathrm{L}}_{n^{\prime}}} L^{\prime}$ est en chaque degré $i$ l'ensemble des familles d'applications $S^{j} \rightarrow L^{\prime i-j}, j$ décrivant $\mathbb{Z}$, telles que pour tout $k \geqslant 1$ et tout $j$, l'application induite $S^{j} \rightarrow\left(L^{\prime} / \mathrm{f}^{k}\right)^{i-j}$, pour la filtration des $\widehat{\mathrm{L}}_{n^{\prime}}$-algèbres, est à support fini, tandis que $L \otimes_{\widehat{\mathrm{L}}_{n}} \mathrm{O}_{n} L^{\prime}$ est en chaque degré $i$ l'ensemble des familles d'applications $S^{j} \rightarrow L^{\prime i-j}$ telles que l'application induite $S^{j} \rightarrow\left(\left(\mathrm{O}_{n} L^{\prime}\right) / \mathrm{f}^{k}\right)^{i-j}$, pour la filtration des $\widehat{\mathrm{L}}_{n}$-algèbres, est à support fini.

Proposition 4.1.19. - Soient $n<n^{\prime} \in \mathbb{N} \cup\{\infty\}, M$ une $\widehat{\mathrm{L}}_{n}$-algèbre et $M^{\prime}$ une $\widehat{\mathrm{L}}_{n^{\prime}-}$ algèbre. On suppose que $M$ admet une présentation $M_{1} \rightarrow M_{0}$ dans $\widehat{\mathcal{L}}_{n}$ avec $M_{1} / \mathrm{f}^{1}$ et $M_{0} / \mathrm{f}^{1}$ finis en chaque degré ou que $M^{\prime}$ est nulle en degré assez grand. Alors le morphisme $M \otimes_{\widehat{\mathrm{L}}_{n}} \mathrm{O}_{n} M^{\prime} \rightarrow \mathrm{O}_{n}\left(\mathrm{M}_{n}^{n^{\prime}}(M) \otimes_{\widehat{\mathrm{L}}_{n^{\prime}}} M^{\prime}\right)$ est un isomorphisme.

Démonstration. - On sait que l'oubli $\mathrm{O}_{n}$ est additif et exact. Le foncteur $\mathrm{M}_{n}^{n^{\prime}}$ est additif et exact à droite car adjoint à gauche de $\mathrm{O}_{n}$. Les produits tensoriels de $\widehat{\mathcal{M}}_{n}$ et $\widehat{\mathcal{M}}_{n^{\prime}}$ sont additifs et exacts à droite en chacune des variables par construction. La proposition se déduit donc du lemme en utilisant des débuts de résolutions libres de $M$ et $M^{\prime}$ satisfaisant aux hypothèses du lemme.

Cas particulier. - Appliquons la proposition à $M^{\prime}=\Lambda_{n^{\prime}}$ qui est nul en degré assez grand. On obtient un isomorphisme naturel $\mathrm{O}_{n} \mathrm{M}_{n}^{n^{\prime}}(M) \simeq M \otimes_{\widehat{\mathrm{L}}_{n}} \mathrm{O}_{n} \Lambda_{n^{\prime}}$. D'autre part $\mathrm{O}_{n} \Lambda_{n^{\prime}}$ est un $\Lambda_{n}$-module libre de dimension finie en chaque degré donc isomorphe à $\widehat{\mathrm{L}}_{n}(S)$, c'est-à-dire à $\mathrm{M}_{0}^{n}\left(\widehat{\mathrm{L}}_{0}(S)\right)$ pour un ensemble $S$ fini en chaque degré. On obtient 
à nouveau par la proposition un isomorphisme naturel $\mathrm{O}_{0}\left(M \otimes_{\widehat{\mathrm{L}}_{n}} \mathrm{M}_{0}^{n}\left(\widehat{\mathrm{L}}_{0}(S)\right)\right) \cong$ $\mathrm{O}_{0} M \otimes_{\widehat{\mathrm{L}}_{0}} \widehat{\mathrm{L}}_{0}(S)$, d'où un isomorphisme

$$
\mathrm{O}_{0} \mathrm{M}_{n}^{n^{\prime}}(M) \cong \mathrm{O}_{0} M \otimes_{\widehat{\mathrm{L}}_{0}} \widehat{\mathrm{L}}_{0}(S)
$$

Proposition 4.1.20. - Soient $M$ une $\widehat{\mathrm{L}}_{0}$-algèbre et $L$ une $\widehat{\mathrm{L}}_{0}$-algèbre libre; alors la $\widehat{\mathrm{L}}_{0}$-algèbre $\operatorname{Tor}_{k}^{\widehat{\mathrm{L}}_{0}}(M, L)$ est nulle pour tout entier $k \geqslant 1$.

Démonstration. - On observe d'abord que $M$ admet une résolution libre de longueur 1. Soit $K$ le noyau d'un épimorphisme $M_{0} \rightarrow M$ avec $M_{0}$ libre. $K$ est un sous-groupe abélien gradué d'un groupe abélien gradué complet pour la filtration $p$-adique et sans torsion donc est également complet pour la filtration $p$-adique et sans torsion donc une $\widehat{\mathrm{L}}_{0}$-algèbre libre par le lemme 4.1.15.

La proposition est maintenant conséquence du résultat plus général suivant :

LEMme 4.1.21. - Soient $n$ un élément de $\mathbb{N} \cup\{\infty\}, M$ une $\widehat{\mathrm{L}}_{n}$-algèbre admettant une résolution libre de longueur 1 et $L$ une $\widehat{\mathrm{L}}_{n}$-algèbre libre; alors la $\widehat{\mathrm{L}}_{n}$-algèbre $\operatorname{Tor}_{k}^{\widehat{\mathrm{L}}_{n}}(M, L)$ est nulle pour tout entier $k \geqslant 1$.

Démonstration. - Puisque $M$ admet une résolution libre de longueur 1 , la $\widehat{\mathrm{L}}_{n}$-algèbre $\operatorname{Tor}_{k}^{\widehat{\mathrm{L}}_{n}}(M, L)$ est nulle pour tout entier $k \geqslant 2$.

Choisissons une résolution libre $0 \rightarrow K \rightarrow M_{0} \rightarrow M$ de longueur 1 de $M$ et un isomorphisme $\widehat{\mathrm{L}}_{0}(S) \rightarrow L$ pour un certain ensemble gradué $S$. Pour tout $L^{\prime} \in \widehat{\mathcal{L}}_{n}$ l'ensemble gradué sous-jacent à $L^{\prime} \otimes_{\widehat{\mathrm{L}}_{0}} L$ s'identifie en chaque degré $i$ à l'ensemble des familles d'applications $S^{j} \rightarrow L^{\prime i-j}, j$ décrivant $\mathbb{Z}$, telles que pour tout $k \geqslant 1$ et pour tout $j$ l'application induite $S^{j} \rightarrow\left(L^{\prime} / \mathrm{f}^{k}\right)^{i-j}$ est à support fini. On en déduit que le morphisme $K \otimes_{\widehat{\mathrm{L}}_{0}} L \rightarrow M_{0} \otimes_{\widehat{\mathrm{L}}_{0}} L$ est injectif en chaque degré, donc que $\operatorname{Tor}_{1}^{\widehat{\mathrm{L}}_{n}}(M, L)$ est nul (lemme B.3).

Corollaire 4.1.22. - Soient $n<n^{\prime} \in \mathbb{N} \cup\{\infty\}$. Le foncteur $\mathrm{M}_{n}^{n^{\prime}}: \widehat{\mathcal{M}}_{n} \rightarrow \widehat{\mathcal{M}}_{n^{\prime}}$ est exact.

Démonstration. - Le corollaire résulte de l'isomorphisme $\mathrm{O}_{0} \mathrm{M}_{n}^{n^{\prime}}(M) \simeq \mathrm{O}_{0} M \otimes_{\widehat{\mathrm{L}}_{0}} L$, pour un $L \in \widehat{\mathcal{L}}_{0}$, observé plus haut et du fait que les foncteurs $\mathrm{O}_{0}$ et $L \otimes_{\widehat{\mathrm{L}}_{0}}-$ sont exacts.

Proposition 4.1.23. - Soient $n<n^{\prime} \in \mathbb{N} \cup\{\infty\}, M$ une $\widehat{\mathrm{L}}_{n}$-algèbre et $M^{\prime}$ une $\widehat{\mathrm{L}}_{n^{\prime}}$-algèbre.

(a) On suppose que $M^{\prime}$ est nul en degré assez grand ou que $M$ admet une présentation $M_{1} \rightarrow M_{0}$ dans $\widehat{\mathcal{L}}_{n}$ avec $M_{1} / \mathrm{f}^{1}$ et $M_{0} / \mathrm{f}^{1}$ finis en chaque degré; alors on a un isomorphisme naturel

$$
\mathrm{O}_{n} \operatorname{Tor}_{*}^{\widehat{\mathrm{L}}_{n^{\prime}}}\left(M^{\prime}, \mathrm{M}_{n}^{n^{\prime}}(M)\right) \cong \operatorname{Tor}_{*}^{\widehat{\mathrm{L}}_{n}}\left(\mathrm{O}_{n} M^{\prime}, M\right) .
$$


(b) On suppose que $M^{\prime}$ est nul en degré assez grand ou que $M$ admet une résolution libre $M_{*} \rightarrow M$ avec $M_{k} / \mathrm{f}^{1}$ fini en chaque degré pour tout $k$; alors on a un isomorphisme naturel

$$
\mathrm{O}_{n} \operatorname{Tor}_{*}^{\widehat{\mathrm{L}}_{n^{\prime}}}\left(\mathrm{M}_{n}^{n^{\prime}}(M), M^{\prime}\right) \cong \operatorname{Tor}_{*}^{\widehat{\mathrm{L}}_{n}}\left(M, \mathrm{O}_{n} M^{\prime}\right) .
$$

Démonstration. - Pour le point (a) soit $M_{*}^{\prime} \rightarrow M^{\prime}$ une résolution libre de $M^{\prime}$. Si $M^{\prime}$ est nul en degré assez grand, on peut choisir $M_{*}^{\prime}$ tel que $M_{k}^{\prime}$ est nul en degré assez grand pour tout $k$. La proposition 4.1.19 montre que les complexes $\mathrm{O}_{n}\left(M_{*}^{\prime} \otimes_{\widehat{\mathrm{L}}_{n^{\prime}}} \mathrm{M}_{n}^{n^{\prime}}(M)\right)$ et $\mathrm{O}_{n}\left(M_{*}^{\prime}\right) \otimes_{\widehat{\mathrm{L}}_{n}} M$ sont naturellement isomorphes donc ont des homologies isomorphes. Comme $\mathrm{O}_{n}$ est exact, l'homologie du complexe $\mathrm{O}_{n}\left(M_{*}^{\prime} \otimes_{\widehat{\mathrm{L}}_{n^{\prime}}} \mathrm{M}_{n}^{n^{\prime}}(M)\right)$ s'identifie à $\mathrm{O}_{n} \operatorname{Tor}_{*}^{\widehat{\mathrm{L}}_{n^{\prime}}}\left(M^{\prime}, \mathrm{M}_{n}^{n^{\prime}}(M)\right)$. Pour la même raison le complexe $\mathrm{O}_{n}\left(M_{*}^{\prime}\right)$ est acyclique. Chaque $\mathrm{O}_{n}\left(M_{k}^{\prime}\right)$ est libre par le lemme 4.1.17 donc l'homologie du complexe $\mathrm{O}_{n}\left(M_{*}^{\prime}\right) \otimes_{\widehat{\mathrm{L}}_{n}} M$ est canoniquement isomorphe à $\operatorname{Tor}_{*}^{\widehat{\mathrm{L}}_{n}}\left(\mathrm{O}_{n} M^{\prime}, M\right)$, d'où le résultat.

Le point (b) se démontre de la même façon en observant que si $M_{*} \rightarrow M$ est une résolution libre de $M$ alors $\mathrm{M}_{n}^{n^{\prime}}\left(M_{*}\right) \rightarrow \mathrm{M}_{n}^{n^{\prime}}(M)$ est une résolution libre de $\mathrm{M}_{n}^{n^{\prime}}(M)$.

Corollaire 4.1.24. - Soient $n \in \mathbb{N} \cup\{\infty\}, M \in \widehat{\mathcal{M}}_{n}$ et $L \in \widehat{\mathcal{L}}_{n}$. On suppose que $M$ est nul en degré assez grand ou que $L / \mathrm{f}^{1}$ est fini en chaque degré; alors la $\widehat{\mathrm{L}}$-algèbre $\operatorname{Tor}_{k}^{\widehat{\mathrm{L}}_{n}}(M, L)$ est nulle pour tout entier $k \geqslant 1$.

Démonstration. - Choisissons un isomorphisme $\widehat{\mathrm{L}}_{n}(S) \cong L$; alors $L$ est isomorphe à $\mathrm{M}_{0}^{n}\left(\widehat{\mathrm{L}}_{0}(S)\right)$ et $S$ est fini en chaque degré si $L / \mathrm{f}^{1}$ est fini en chaque degré. L'objet $\mathrm{O}_{0} \operatorname{Tor}_{k}^{\widehat{\mathrm{L}}_{n}}(M, L)$ est isomorphe à $\operatorname{Tor}_{k}\left(\mathrm{O}_{0} M, \mathrm{M}_{0}^{n}\left(\widehat{\mathrm{L}}_{0}(S)\right)\right)$ donc est nul par la proposition 4.1.20.

La proposition suivante est le pendant de la proposition 4.1 .8 et se démontre de la même façon :

Proposition 4.1.25. - Soit $M$ une $\widehat{\mathrm{L}}_{n}$-algèbre et $k$ un entier positif. Les conditions suivantes sont équivalentes :

(i) La $\widehat{\mathrm{L}}_{n}$-algèbre $\operatorname{Tor}_{k+1}^{\widehat{\mathrm{L}}_{n}}\left(M, \Lambda_{n} / \mathrm{f}^{1}\right)$ est nulle.

(ii) Il existe une résolution libre de $M$ de longueur inférieure ou égale à $k$.

Si elles sont vérifiées, la $\widehat{\mathrm{L}}_{n}$-algèbre $\operatorname{Tor}_{l}^{\widehat{\mathrm{L}}_{n}}\left(M, \Lambda_{n} / \mathrm{f}^{1}\right)$ est nulle pour tout entier $l \geqslant$ $k+1$.

Proposition 4.1.26. - Soient $n<\infty$ un entier et $M$ une $\widehat{\mathrm{L}}_{n}$-algèbre; alors $M$ admet une résolution libre de longueur $n+1$.

Démonstration. - On montre par récurrence sur $n$ que l'objet $\operatorname{Tor}_{k}^{\widehat{\mathrm{L}}_{n}}\left(M, \Lambda_{n} / \mathrm{f}^{1}\right)$ est nul pour tout $k \geqslant n+2$, ce qui permet de conclure par la proposition qui précède. 
Pour $n=0$ soit $M_{0} \rightarrow M$ un épimorphisme avec $M_{0}$ dans $\widehat{\mathcal{L}}_{0}$, alors le noyau de $M_{0} \rightarrow M$ est complet pour la filtration $p$-adique et sans torsion, donc est dans $\widehat{\mathcal{L}}_{0}$.

Supposons le résultat vrai pour $n$. La $\widehat{\mathrm{L}}_{n+1^{-}}$-algèbre $\mathrm{M}_{n}^{n+1}\left(\Lambda_{n} / \mathrm{f}^{1}\right)$ est un $\Lambda_{n+1^{-}}$ module complet pour la filtration par les coefficients qui s'identifie au module sousjacent à la $\mathbb{Z} / p$-algèbre graduée $\mathbb{Z} / p\left[x_{n+1}\right]$, où $x_{n+1}$ est un générateur de degré $2 n+2$. On a donc une suite exacte de $\widehat{\mathrm{L}}_{n+1}$-algèbres

$$
0 \longrightarrow \mathrm{M}_{n}^{n+1}\left(\Lambda_{n} / \mathrm{f}^{1}\right) \longrightarrow \mathrm{M}_{n}^{n+1}\left(\Lambda_{n} / \mathrm{f}^{1}\right) \longrightarrow \Lambda_{n+1} / \mathrm{f}^{1} \longrightarrow 0 .
$$

Puisque l'objet $\operatorname{Tor}_{1}^{\widehat{\mathrm{L}}_{n+1}}\left(\Lambda_{n+1} / \mathrm{f}^{1}, L\right)$ est nul pour tout $L \in \widehat{\mathcal{L}}_{n+1}$ par le corollaire 4.1.24, la suite exacte courte ci-dessus induit pour tout entier $k$ une suite exacte $\operatorname{Tor}_{k+1}^{\widehat{\mathrm{L}}_{n+1}}\left(M, \mathrm{M}_{n}^{n+1}\left(\Lambda_{n} / \mathrm{f}^{1}\right)\right) \longrightarrow \operatorname{Tor}_{k+1}^{\widehat{\mathrm{L}}_{n+1}}\left(M, \Lambda_{n+1} / \mathrm{f}^{1}\right) \longrightarrow \operatorname{Tor}_{k}^{\widehat{\mathrm{L}}_{n+1}}\left(M, \mathrm{M}_{n}^{n+1}\left(\Lambda_{n} / \mathrm{f}^{1}\right)\right)$. La $\widehat{\mathrm{L}}_{n}$-algèbre $\mathrm{O}_{n} \operatorname{Tor}_{k}^{\widehat{\mathrm{L}}_{n+1}}\left(M, \mathrm{M}_{n}^{n+1}\left(\Lambda_{n} / \mathrm{f}^{1}\right)\right)$ s'identifie à $\operatorname{Tor}_{k}^{\widehat{\mathrm{L}}_{n}}\left(\mathrm{O}_{n} M, \Lambda_{n} / \mathrm{f}^{1}\right)$ par la proposition 4.1.23 donc est nulle pour $k \geqslant n+2$ par hypothèse de récurrence. On en déduit que la $\widehat{\mathrm{L}}_{n+1}$-algèbre $\operatorname{Tor}_{k} \widehat{\mathrm{L}}_{n+1}\left(M, \Lambda_{n+1} / \mathrm{f}^{1}\right)$ est nulle pour tout $k \geqslant n+3$.

Nous dirons qu'une $\widehat{\mathrm{L}}$-algèbre $M$ est de présentation en degré borné si $M$ est le conoyau d'un morphisme $M_{1} \rightarrow M_{0}$ de $\widehat{\mathcal{L}}$ avec $M_{1} / \mathrm{f}^{1}$ et $M_{0} / \mathrm{f}^{1}$ concentrés en un nombre fini de degrés. La MU-cohomologie continue d'un espace profini de dimension finie en est un exemple.

Proposition 4.1.27. - Soient $M \rightarrow M^{\prime}$ un morphisme entre $\widehat{\mathrm{L}}$-algèbres de présentation en degré borné; alors il existe un entier $n$, un morphisme $N \rightarrow N^{\prime}$ entre $\widehat{\mathrm{L}}_{n}$-algèbres et un isomorphisme de $M \rightarrow M^{\prime}$ sur le morphisme $\mathrm{M}_{n}^{\infty}(N) \rightarrow \mathrm{M}_{n}^{\infty}\left(N^{\prime}\right)$.

Démonstration. - Choisissons des présentations $\widehat{\mathrm{L}}\left(S_{1}\right) \rightarrow \widehat{\mathrm{L}}\left(S_{0}\right) \rightarrow M$ et $\widehat{\mathrm{L}}\left(S_{1}^{\prime}\right) \rightarrow$ $\widehat{\mathrm{L}}\left(S_{0}^{\prime}\right) \rightarrow M^{\prime}$ de $M$ et $M^{\prime}$ avec $S_{1}, S_{0}, S_{1}^{\prime}, S_{0}^{\prime}$ concentrés en un nombre fini de degrés. Le morphisme $M \rightarrow M^{\prime}$ se relève en un morphisme de $\widehat{\mathrm{L}}\left(S_{1}\right) \rightarrow \widehat{\mathrm{L}}\left(S_{0}\right)$ dans $\widehat{\mathrm{L}}\left(S_{1}^{\prime}\right) \rightarrow$ $\widehat{\mathrm{L}}\left(S_{0}^{\prime}\right)$. Soit $n$ un entier supérieur à la moitié de la différence entre le degré maximal et le degré minimal d'un élément de $S_{1} \cup S_{0} \cup S_{1}^{\prime} \cup S_{0}^{\prime}$. Le carré commutatif

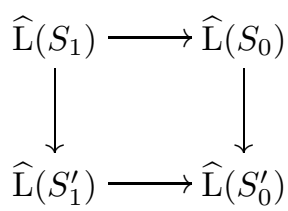

est alors l'image par $\mathrm{M}_{n}^{\infty}$ d'un carré

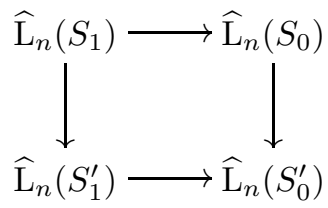


dans $\widehat{\mathcal{M}}_{n}$ et ce dernier commute puisque la restriction de $\mathrm{M}_{n}^{\infty}$ à $\widehat{\mathcal{L}}_{n}$ est fidèle. Notons $N$ et $N^{\prime}$ les conoyaux des morphismes $\widehat{\mathrm{L}}_{n}\left(S_{1}\right) \rightarrow \widehat{\mathrm{L}}_{n}\left(S_{0}\right)$ et $\widehat{\mathrm{L}}_{n}\left(S_{1}^{\prime}\right) \rightarrow \widehat{\mathrm{L}}_{n}\left(S_{0}^{\prime}\right)$ respectivement. Le morphisme $\widehat{\mathrm{L}}_{n}\left(S_{0}\right) \rightarrow \widehat{\mathrm{L}}_{n}\left(S_{0}^{\prime}\right)$ induit un morphisme $N \rightarrow N^{\prime}$ d'image par $\mathrm{M}_{n}^{\infty}$ isomorphe à $M \rightarrow N$.

Proposition 4.1.28. - Soit $M$ une $\widehat{\mathrm{L}}$-algèbre de présentation en degré borné, alors $M$ admet une résolution libre de longueur finie.

Démonstration. - La proposition est conséquence de la proposition ci-dessus appliquée à l'identité de $M$, de la proposition 4.1.26, de l'exactitude du foncteur $\mathrm{M}_{n}^{\infty}$ et $\mathrm{du}$ fait que $\mathrm{M}_{n}^{\infty}$ transforme les $\widehat{\mathrm{L}}_{n}$-algèbres libres en $\widehat{\mathrm{L}}$-algèbres libres.

Produit tensoriel et limite. — Soit $\left(N_{s}, g_{s}: N_{s} \rightarrow N_{s-1}\right)$ une tour de $\widehat{\text { L-algèbres dont }}$ on note $N_{\infty}$ la limite; alors $N_{\infty}$ est le noyau du morphisme Id $-\left(g_{s}\right): \prod_{s} N_{s} \rightarrow \prod_{s} N_{s}$ et $\lim _{s}^{1} N_{s}$ son conoyau (appendice C).

Soient $M$ une $\widehat{\mathrm{L}}$-algèbre et $M_{*} \rightarrow M$ une résolution libre. On forme le bicomplexe $A_{*, *}$ concentré en bidegré $(*, 1)$ et $(*, 0)$ et défini par

$$
A_{*, 1} \longrightarrow A_{*, 0}=\prod_{t}\left(M_{*} \widehat{\otimes} N_{t}\right) \longrightarrow \prod_{t}\left(M_{*} \widehat{\otimes} N_{t}\right)
$$

de sorte qu'on a avec les notations de l'appendice $\mathrm{C}$

$$
\mathrm{H}_{1}^{v} A_{s, *}=\lim _{t}\left(M_{s} \widehat{\otimes} N_{t}\right), \mathrm{H}_{0}^{v} A_{s, *}=\lim _{t}^{1}\left(M_{s} \widehat{\otimes} N_{t}\right) .
$$

Le morphisme $M \widehat{\otimes} \prod_{t} N_{t} \rightarrow \prod_{t}\left(M \widehat{\otimes} N_{t}\right)$ induit des morphismes

$$
M \widehat{\otimes} N_{\infty} \longrightarrow \lim _{t}\left(M \widehat{\otimes} N_{t}\right) \quad \text { et } \quad M \widehat{\otimes} \lim _{t}^{1} N_{t} \longrightarrow \lim _{t}^{1}\left(M \widehat{\otimes} N_{t}\right) .
$$

LEMME 4.1.29. - Soient $M$ une $\widehat{\mathrm{L}}$-algèbre et $\left(L_{s}\right)$ une tour de $\widehat{\mathcal{L}}$ telle que la tour de $\mathbb{F}_{p}$-espaces vectoriels gradués $\left(L_{s} / \mathrm{f}^{1}\right)$ est pro-constante en chaque degré. Alors le morphisme $M \widehat{\otimes} L_{\infty} \rightarrow \lim _{s}\left(M \widehat{\otimes} L_{s}\right)$ est un isomorphisme et $\lim _{s}^{1}\left(M \widehat{\otimes} L_{s}\right)$ est nul.

Démonstration. - On commence par traiter le cas $M \in \widehat{\mathcal{L}}$. Puisque $\lim _{s}^{1}\left(L_{s} / \mathrm{f}^{1}\right)$ est nul, $L_{\infty}$ est dans $\widehat{\mathcal{L}}$ et $\lim _{s}^{1} L_{s}$ est nul par le lemme 2.1.12. De même, pour chaque entier $t$ la $\widehat{\mathrm{L}}$-algèbre $\lim _{s}\left(L_{s} / \mathrm{F}^{t}\right)$ est dans $\widehat{\mathcal{L}}$. Le lemme 2.1 .12 montre que le morphisme $L_{\infty} / \mathrm{F}^{t} \rightarrow \lim _{s}\left(L_{s} / \mathrm{F}^{t}\right)$ est un isomorphisme modulo $\mathrm{f}^{1}$ donc (corollaire 2.1.4) c'est un isomorphisme dans $\widehat{\mathcal{L}}$.

Les morphismes $M \widehat{\otimes} L_{\infty} \rightarrow \lim _{t}\left(M / \mathrm{F}^{t} \widehat{\otimes} L_{\infty} / \mathrm{F}^{t}\right)$ et $M \widehat{\otimes} L_{s} \rightarrow \lim _{t}\left(M / \mathrm{F}^{t} \widehat{\otimes} L_{s} / \mathrm{F}^{t}\right)$ sont des isomorphismes par construction du produit tensoriel. Pour chaque entier $t$ le lemme 2.1.12 appliqué à la tour $\left(M / \mathrm{F}^{t} \widehat{\otimes} L_{s} / \mathrm{F}^{t}\right)_{s}$ et le corollaire 2.1.4 montrent que le morphisme $M / \mathrm{F}^{t} \widehat{\otimes} L_{\infty} / \mathrm{F}^{t} \rightarrow \lim _{s}\left(M / \mathrm{F}^{t} \widehat{\otimes} L_{s} / \mathrm{F}^{t}\right)$ est un isomorphisme dans $\widehat{\mathcal{L}}$ et que $\lim _{s}^{1}\left(M / \mathrm{F}^{t} \widehat{\otimes} L_{s} / \mathrm{F}^{t}\right)$ est nul. Les mêmes lemme et corollaire donnent un isomorphisme $\lim _{t}\left(M / \mathrm{F}^{t} \widehat{\otimes} L_{\infty} / \mathrm{F}^{t}\right) \rightarrow \lim _{s, t}\left(M / \mathrm{F}^{t} \widehat{\otimes} L_{s} / \mathrm{F}^{t}\right)$. On en déduit un isomorphisme naturel $M \widehat{\otimes} L_{\infty} \cong \lim _{s}\left(M \widehat{\otimes} L_{s}\right)$. Enfin la proposition C.7 appliquée à la tour $\left(M / \mathrm{F}^{t} \widehat{\otimes} L_{s} / \mathrm{F}^{t}\right)_{s, t}$ et les résultats qui précèdent montrent que la $\widehat{\mathrm{L}}$-algèbre $\lim _{s}^{1}\left(M \widehat{\otimes} L_{s}\right)$ est nulle. 
Pour $M$ quelconque soit $M_{1} \rightarrow M_{0} \rightarrow M$ le début d'une résolution libre de $M$. Par exactitude à droite du foncteur $\lim ^{1}$, la $\widehat{\mathrm{L}}$-algèbre $\lim ^{1}\left(M \widehat{\otimes} L_{s}\right)$ est nulle. Par exactitude du produit tensoriel et parce que $\lim _{s}^{1}\left(M_{1} \widehat{\otimes} L_{s}\right)$ est nul, on a une suite exacte $\lim _{s}\left(M_{1} \widehat{\otimes} L_{s}\right) \rightarrow \lim _{s}\left(M_{0} \widehat{\otimes} L_{s}\right) \rightarrow \lim _{s}\left(M \widehat{\otimes} L_{s}\right) \rightarrow 0$. En comparant avec la suite exacte $M_{1} \widehat{\otimes} L_{\infty} \rightarrow M_{0} \widehat{\otimes} L_{\infty} \rightarrow M \widehat{\otimes} L_{\infty} \rightarrow 0$ on déduit que le morphisme $M \widehat{\otimes} L_{\infty} \rightarrow \lim _{s}\left(M \widehat{\otimes} L_{s}\right)$ est un isomorphisme.

Lemme 4.1.30. - Soit $M$ une $\widehat{\mathrm{L}}$-algèbre et $\left(N_{\alpha}\right)$ une famille de $\widehat{\mathrm{L}}$-algèbres. On suppose que $M$ est le conoyau d'un morphisme $M_{1} \rightarrow M_{0}$ de $\widehat{\mathcal{L}}$ avec $M_{1} / \mathrm{f}^{1}$ et $M_{0} / \mathrm{f}^{1}$ finis en chaque degré; alors le morphisme

$$
M \widehat{\otimes} \prod_{\alpha} N_{\alpha} \longrightarrow \prod_{\alpha}\left(M \widehat{\otimes} N_{\alpha}\right)
$$

est un isomorphisme.

Démonstration. - Supposons d'abord $M$ dans $\widehat{\mathcal{L}}$ et $M / \mathrm{f}^{1}$ de dimension totale finie. Il existe alors un ensemble gradué $S$ fini en chaque degré et vide sauf en un nombre fini de degrés, et un isomorphisme $\widehat{\mathrm{L}}(S) \rightarrow M$. Pour toute $\widehat{\mathrm{L}}$-algèbre $N$ le produit tensoriel $M \widehat{\otimes} N$ s'écrit comme le produit $\prod_{s \in S} \Sigma^{|s|} N$ (voir l'appendice B). Le foncteur $N \mapsto \Sigma^{|s|} N$ commute aux produits quelconques, d'où le résultat.

Supposons $M$ dans $\widehat{\mathcal{L}}$ avec $M / \mathrm{f}^{1}$ fini en chaque degré. Notons pour $s \geqslant 0 M_{s}$ la sous-L̂L-algèbre de $M$ engendrée par les éléments de degré compris entre $-s$ et $s$; alors $\left(M_{s}\right)$ est une tour d'objets de $\widehat{\mathcal{L}}$ vérifiant les hypothèses du lemme 4.1 .29 , a pour limite $M$ et est telle que $M_{s} / \mathrm{f}^{1}$ est de dimension totale finie pour tout $s$. On en déduit des isomorphismes

$$
M \widehat{\otimes} \prod_{\alpha} N_{\alpha} \cong \lim _{s}\left(M_{s} \widehat{\otimes} \prod_{\alpha} N_{\alpha}\right) \cong \lim _{s, \alpha}\left(M_{s} \widehat{\otimes} N_{\alpha}\right) \cong \prod_{\alpha}\left(M \widehat{\otimes} N_{\alpha}\right) .
$$

Le cas général s'obtient par commutation du produit tensoriel et des produits au conoyau.

Proposition 4.1.31. - Soient $\left(N_{s}\right)$ une tour de $\widehat{\mathrm{L}}$-algèbres, $M$ une $\widehat{\mathrm{L}}$-algèbre et $M_{*} \rightarrow M$ une résolution libre de $M$. On suppose que l'une des conditions suivantes est satisfaite :

(i) Pour tout $n \in \mathbb{N}, M_{n} / \mathrm{f}^{1}$ est fini en chaque degré.

(ii) La tour $\left(N_{s}\right)$ est le conoyau d'un morphisme $\left(N_{1, s} \rightarrow N_{0, s}\right)$ entre tours d'objets de $\widehat{\mathcal{L}}$ avec $\left(N_{1, s} / \mathrm{f}^{1}\right)$ et $\left(N_{0, s} / \mathrm{f}^{1}\right)$ pro-constantes en chaque degré.

Alors :

(a) On a un isomorphisme $M \widehat{\otimes} \lim _{s}^{1} N_{s} \rightarrow \lim _{s}^{1}\left(M \widehat{\otimes} N_{s}\right)$.

(b) Supposons $\lim _{s}^{1} N_{s}=0$ alors on a pour tout entier $n$ une suite exacte

$$
0 \longrightarrow \lim _{s}^{1} \operatorname{Tor}_{n+1}\left(M, N_{s}\right) \longrightarrow \operatorname{Tor}_{n}\left(M, N_{\infty}\right) \longrightarrow \lim _{s} \operatorname{Tor}_{n}\left(M, N_{s}\right) \longrightarrow 0 .
$$


(c) Supposons que la tour $\left(\operatorname{Tor}_{n}\left(M, N_{s}\right)\right)_{s}$ est de limite et de $\lim ^{1}$ nuls pour tout entier $n \geqslant 1$ alors on a une suite exacte

$$
0 \longrightarrow \operatorname{Tor}_{2}\left(M, \lim _{s}^{1} N_{s}\right) \longrightarrow M \widehat{\otimes} N_{\infty} \longrightarrow \lim _{s}\left(M \widehat{\otimes} N_{s}\right) \longrightarrow \operatorname{Tor}_{1}\left(M, \lim _{s}^{1} N_{s}\right) \longrightarrow 0
$$

et un isomorphisme $\operatorname{Tor}_{n+2}\left(M, \lim _{s}^{1} N_{s}\right) \simeq \operatorname{Tor}_{n}\left(M, N_{\infty}\right)$ pour tout $n \geqslant 1$.

Démonstration. - On montre d'abord que si $M$ est dans $\widehat{\mathcal{L}}$ et si l'une des conditions (i) ou (ii) est vérifiée alors les morphismes $M \widehat{\otimes} N_{\infty} \rightarrow \lim _{s}\left(M \widehat{\otimes} N_{s}\right)$ et $M \widehat{\otimes} \lim _{s}^{1} N_{s} \rightarrow$ $\lim _{s}^{1}\left(M \widehat{\otimes} N_{s}\right)$ sont des isomorphismes.

Supposons $M$ dans $\widehat{\mathcal{L}}$ et $M / \mathrm{f}^{1}$ fini en chaque degré; alors le morphisme

$$
M \widehat{\otimes} \prod_{s} N_{s} \longrightarrow \prod_{s}\left(M \widehat{\otimes} N_{s}\right)
$$

est un isomorphisme par le lemme 4.1.30. Comme le produit tensoriel par $M$ est exact (corollaire 4.1.24), on en déduit que les morphismes $M \widehat{\otimes} N_{\infty} \rightarrow \lim _{s}\left(M \widehat{\otimes} N_{s}\right)$ et $M \widehat{\otimes} \lim _{s}^{1} N_{s} \rightarrow \lim _{s}^{1}\left(M \widehat{\otimes} N_{s}\right)$ sont des isomorphismes.

Supposons que $M$ est dans $\widehat{\mathcal{L}}$ et que $\left(N_{s}\right)$ vérifie la condition (ii). Alors $\lim _{s}^{1} N_{s}$ est nul par exactitude à droite de $\lim ^{1}$ et on a une suite exacte $N_{1, \infty} \rightarrow N_{0, \infty} \rightarrow N_{\infty} \rightarrow 0$. La L-algèbre $\lim _{s}^{1}\left(M \widehat{\otimes} N_{i, s}\right)$ est nulle pour $i=0,1$ par le lemme $\operatorname{donc} \lim _{s}^{1}\left(M \widehat{\otimes} N_{s}\right)$ est nul et on a une suite exacte $\lim _{s}\left(M \widehat{\otimes} N_{1, s}\right) \rightarrow \lim _{s}\left(M \widehat{\otimes} N_{0, s}\right) \rightarrow \lim _{s}\left(M \widehat{\otimes} N_{s}\right) \rightarrow 0$. Le morphisme $M \widehat{\otimes} N_{i, \infty} \rightarrow \lim _{s}\left(M \widehat{\otimes} N_{i, s}\right)$ est un isomorphisme pour $i=0,1$ par le lemme donc induit un isomorphisme $M \widehat{\otimes} N_{\infty} \rightarrow \lim _{s}\left(M \widehat{\otimes} N_{s}\right)$.

On démontre maintenant la proposition en appliquant la proposition C.6 au bicomplexe $A_{*, *}$ associé à la tour $\left(N_{s}\right)$ et à la résolution libre $M_{*} \rightarrow M$. Les termes $\mathrm{H}_{0}^{v} \mathrm{H}_{s}^{h} A$ et $\mathrm{H}_{1}^{v} \mathrm{H}_{s}^{h} A$ s'identifient respectivement à $\lim _{t}^{1} \operatorname{Tor}_{s}\left(M, N_{t}\right)$ et $\lim _{t} \operatorname{Tor}_{s}\left(M, N_{t}\right)$. D'autre part si la condition (i) ou (ii) est vérifiée alors les termes $\mathrm{H}_{s}^{h} \mathrm{H}_{1}^{v} A$ et $\mathrm{H}_{s}^{h} \mathrm{H}_{0}^{v} A$ s'identifient respectivement d'après ce qui précède à $\operatorname{Tor}_{s}\left(M, \mathrm{~N}_{\infty}\right)$ et $\operatorname{Tor}_{s}\left(M, \lim _{t}^{1} N_{t}\right)$, ce qui permet de conclure.

Application : filtration des $\widehat{\mathrm{L}}$-algèbres et limites. - On applique ce qui précède à la $\widehat{\mathrm{L}}$-algèbre $\widehat{\mathrm{MU}}^{*} / \mathrm{f}^{n}$ et une tour de $\widehat{\mathrm{L}}$-algèbres $\left(M_{s}\right)$. La $\widehat{\mathrm{L}}$-algèbre $\widehat{\mathrm{MU}}^{*} / \mathrm{f}^{n}$ vérifie la condition (i) de la proposition par le corollaire 4.1.11.

Proposition 4.1.32

(a) On a pour toute $\widehat{\mathrm{L}}$-algèbre $M$ un isomorphisme canonique $M \widehat{\otimes} \widehat{\mathrm{MU}}^{*} / \mathrm{f}^{n} \cong$ $M / \mathrm{f}^{n}$.

(b) On a pour toute $\widehat{\mathrm{L}}$-algèbre $M$ et tout entier $k$ un isomorphisme

$$
\operatorname{Tor}_{k}\left(M, \widehat{\mathrm{MU}}^{*} / \mathrm{f}^{n}\right) \cong \operatorname{Tor}_{k}\left(\widehat{\mathrm{MU}}^{*} / \mathrm{f}^{n}, M\right) \text {. }
$$

(c) Soit $\left(M_{s}\right)$ une tour de $\widehat{\mathrm{L}}$-algèbres; alors on a l'équivalence

$$
\lim _{s}^{1} M_{s}=0 \Longleftrightarrow \lim _{s}^{1}\left(M_{s} / \mathrm{f}^{1}\right)=0 .
$$


(d) Soit $\left(M_{s}\right)$ une tour de $\widehat{\mathrm{L}}$-algèbres telle que $\lim _{s}^{1} M_{s}$ est nul; alors on a des suites exactes

$$
0 \longrightarrow \lim _{s}^{1} \operatorname{Tor}_{1}\left(M_{s}, \widehat{\mathrm{MU}}^{*} / \mathrm{f}^{n}\right) \longrightarrow M_{\infty} / \mathrm{f}^{n} \longrightarrow \lim _{s}\left(M_{s} / \mathrm{f}^{n}\right) \longrightarrow 0
$$

et pour $k \geqslant 1$

$$
\begin{aligned}
& 0 \longrightarrow \lim _{s}^{1} \operatorname{Tor}_{k+1}\left(M_{s}, \widehat{\mathrm{MU}}^{*} / \mathrm{f}^{n}\right) \longrightarrow \operatorname{Tor}_{k}\left(M_{\infty}, \widehat{\mathrm{MU}}^{*} / \mathrm{f}^{n}\right) \\
& \longrightarrow \lim _{s} \operatorname{Tor}_{k}\left(M_{s}, \widehat{\mathrm{MU}}^{*} / \mathrm{f}^{n}\right) \longrightarrow 0
\end{aligned}
$$

Démonstration. - Pour le point (a) supposons d'abord que $M$ est dans $\widehat{\mathcal{L}}$ et est nulle en degré assez grand; alors la filtration de $M$ est la filtration par les coefficients et l'isomorphisme $M \widehat{\otimes} \widehat{M U}^{*} / \mathrm{f}^{n} \cong M / \mathrm{f}^{n}$ est une traduction de l'isomorphisme $M \widehat{\otimes} \widehat{\mathrm{MU}}^{*} / \mathrm{f}^{n} \cong M \otimes \widehat{\mathrm{MU}}^{*} \widehat{\mathrm{MU}}^{*} / \mathrm{f}^{n}$ donné par la proposition 2.2.11.

Le cas $M \in \widehat{\mathcal{L}}$ quelconque s'obtient en appliquant le point (b) de la proposition 4.1.31 à la $\widehat{\mathrm{L}}$-algèbre $\widehat{\mathrm{MU}}^{*} / \mathrm{f}^{n}$ et à la tour $\left(M / \mathrm{F}^{s}\right)$. Le cas général s'obtient en utilisant le début d'une résolution libre de $M$ et l'exactitude à droite des foncteurs $N \mapsto$ $N \widehat{\otimes} \widehat{\mathrm{MU}}^{*} / \mathrm{f}^{n}$ et $N \mapsto N / \mathrm{f}^{n}$.

Pour le point (b) on observe d'abord que $\widehat{\mathrm{MU}}^{*} / \mathrm{f}^{n}$ admet une résolution libre $N_{*} \rightarrow$ $\widehat{\mathrm{MU}}^{*} / \mathrm{f}^{n}$ telle que $N_{s} / \mathrm{f}^{1}$ est fini en chaque degré. Soit alors $M$ une $\widehat{\mathrm{L}}$-algèbre. La $\widehat{\mathrm{L}}$ algèbre $\operatorname{Tor}_{k}\left(M, N_{l}\right)$ est nulle pour tout $k \geqslant 1$ et $l \geqslant 0$ par le corollaire 4.1.24. Ce même corollaire montre que $\operatorname{Tor}_{k}\left(\widehat{\mathrm{MU}}^{*} / \mathrm{f}^{n}, L\right)$ est nul pour tout $k \geqslant 1$ et tout $L \in \widehat{\mathcal{L}}$. Le point (b) est maintenant conséquence du corollaire B.5.

Pour le point (c) on a un isomorphisme $\left(\lim _{s}^{1} M_{s}\right) / \mathrm{f}^{1} \cong \lim _{s}^{1}\left(M_{s} / \mathrm{f}^{1}\right)$ par le point (a) ci-dessus et le point (a) de la proposition 4.1.31. Le lemme 2.2.3 permet de conclure.

Le point (d) est une traduction du point (b) de la proposition 4.1.31 au vu des points (a) et (b).

\section{EXEMPles}

- Soit $\left(M_{s}\right)$ une tour de $\widehat{\mathrm{L}}$-algèbres telle que $M_{s} / \mathrm{f}^{1}$ est fini en chaque degré et nul en degré assez grand. Chaque $\widehat{\mathrm{L}}$-algèbre $M_{s}$ admet une résolution par des objets de $\widehat{\mathcal{L}}$ nuls en degré assez grand et finis en chaque degré modulo $\mathrm{f}^{1}$ par le corollaire 4.1.11 de sorte que pour tout entier $k$ l'objet $\operatorname{Tor}_{k}\left(M_{s}, \widehat{\mathrm{MU}}^{*} / \mathrm{f}^{n}\right)$ est un $\widehat{\mathrm{MU}}^{*} / \mathrm{f}^{n}$-module fini en chaque degré. On en déduit que la $\widehat{\mathrm{L}}$-algèbre $\lim _{s}^{1} \operatorname{Tor}_{k}\left(M_{s}, \widehat{\mathrm{MU}}^{*} / \mathrm{f}^{n}\right)$ est nulle, d'où un isomorphisme

$$
\operatorname{Tor}_{k}\left(M_{\infty}, \widehat{\mathrm{MU}}^{*} / \mathrm{f}^{n}\right) \cong \lim _{s} \operatorname{Tor}_{k}\left(M_{s}, \widehat{\mathrm{MU}}^{*} / \mathrm{f}^{n}\right)
$$

En particulier la filtration de $M_{\infty}$ est la filtration limite de celle des $M_{s}$. Ce résultat se généralise au cas où $M_{s}$ a une structure profinie naturelle.

- Soit $X$ un ensemble simplicial, alors les $\widehat{\mathrm{L}}$-algèbres $\operatorname{Tor}_{k}\left(\widehat{\mathrm{MU}}^{*} / \mathrm{f} n, \widehat{\mathrm{MU}}^{*} \mathrm{Sk}_{s} X\right)$ ont une structure profinie naturelle donc sont sans $\lim ^{1}$ par rapport à $s$. On en déduit pour tout $k$ un isomorphisme

$$
\operatorname{Tor}_{k}\left(\widehat{\mathrm{MU}}^{*} X, \widehat{\mathrm{MU}}^{*} / \mathrm{f}^{n}\right) \cong \lim _{s} \operatorname{Tor}_{k}\left(\widehat{\mathrm{MU}}^{*} \mathrm{Sk}_{s} X, \widehat{\mathrm{MU}}^{*} / \mathrm{f}^{n}\right)
$$




\subsection{Résolutions instables et résolutions des espaces}

Résolution instable. — La structure de monade du foncteur $\mathrm{G}: S \mapsto \widehat{\mathrm{MU}}^{*} \mathrm{~K}(S)$ sur $\mathcal{E}$ ns-gr permet d'associer à toute $M U$-algèbre instable $M$ sa G-résolution simpliciale canonique $\mathrm{G}_{\bullet}(M) \rightarrow M$ (voir la section A.4 de l'appendice). Comme le morphisme de $\mathrm{G}_{\bullet}(M)$ dans l'objet simplicial constant $M$ est une équivalence d'homotopie entre objets simpliciaux de $\mathcal{E}$ ns-gr, le complexe de $\widehat{\mathrm{L}}$-algèbres $\mathrm{G}_{*}(M) \rightarrow M$ est acyclique. Comme la $\widehat{\mathrm{L}}$-algèbre sous-jacente à $\mathrm{G}_{n}(M)$ est libre pour tout $n$, le complexe $\mathrm{G}_{*}(M) \rightarrow M$ est une résolution libre de la $\widehat{\mathrm{L}}$-algèbre sous-jacente à $M$.

La structure de monade du foncteur $X \mapsto \mathrm{K}\left(\widehat{\mathrm{MU}}^{*} X\right)$ sur h $\widehat{\mathcal{S}}$ permet de même d'associer à tout espace profini $X$ un objet cosimplicial de $\mathrm{h} \widehat{\mathcal{S}}$, noté $\mathrm{K} \cdot\left(\widehat{\mathrm{MU}}^{*} X\right)$, et une augmentation $X \rightarrow \mathrm{K}^{\bullet}\left(\widehat{\mathrm{MU}}^{*} X\right)$. L'image en MU-cohomologie continue de l'objet cosimplicial augmenté $X \rightarrow \mathrm{K}^{\bullet}\left(\widehat{\mathrm{MU}}^{*} X\right)$ est la G-résolution simpliciale canonique de $\widehat{\mathrm{MU}}^{*} X$. On appelle $X \rightarrow \mathrm{K}^{\bullet}\left(\widehat{\mathrm{MU}}^{*} X\right)$ la MU-résolution cosimpliciale canonique de $X$.

Observons que l'objet simplicial $\mathrm{G}_{\bullet}(M)$ associé à une MU-algèbre instable $M$ est canoniquement l'image en MU-cohomologie continue d'un objet cosimplicial de h $\widehat{\mathcal{S}}$ qu'on note $\mathrm{K}^{\bullet}(M)$.

Rappelons qu'on note $\pi_{0}\left(M_{\bullet}\right)$ le coégalisateur du 1-complexe $M_{1} \leftrightarrows M_{0}$ issu d'un objet simplicial $M_{\bullet}$. Le foncteur $M_{\bullet} \mapsto \pi_{0}\left(M_{\bullet}\right)$ commute aux foncteurs oubli $\mathcal{K}_{\mathrm{MU}} \rightarrow$ $\widehat{\mathcal{M}}$ et $\mathcal{K}_{\mathrm{H}} \rightarrow \mathcal{E}$ et au foncteur $M \mapsto M / \mathrm{f}^{1}: \widehat{\mathcal{M}} \rightarrow \mathcal{E}$. La proposition suivante est une traduction de la proposition 4.1 .3 :

Proposition 4.2.1. - Soit $M_{\bullet}$ une MU-algèbre instable simpliciale telle que $M_{n}$ est dans $\widehat{\mathcal{L}}$ pour tout entier $n$; alors :

(a) Les conditions suivantes sont équivalentes :

(i) La $\mathrm{HZ} / p$-algèbre simpliciale augmentée $M_{\bullet} / \mathrm{f}^{1} \rightarrow \pi_{0}\left(M_{\bullet} / \mathrm{f}^{1}\right)$ est acyclique comme objet simplicial augmenté de $\mathcal{E}$.

(ii) La $\widehat{\mathrm{L}}$-algèbre sous-jacente à $\pi_{0}\left(M_{\bullet}\right)$ est dans $\widehat{\mathcal{L}}$ et $M_{\bullet} \rightarrow \pi_{0}\left(M_{\bullet}\right)$ est acyclique comme objet simplicial de $\widehat{\mathcal{M}}$.

(b) Soient $M$ une MU-algèbre instable libre comme $\widehat{\mathrm{L}}$-algèbre et $\pi_{0}\left(M_{\bullet}\right) \rightarrow M$ un morphisme dans $\mathcal{K}_{\mathrm{MU}}$; alors $\pi_{0}\left(M_{\bullet}\right) \rightarrow M$ est un isomorphisme si et seulement si le morphisme induit $\pi_{0}\left(M_{\bullet} / \mathrm{f}^{1}\right) \rightarrow M / \mathrm{f}^{1}$ est un isomorphisme.

Corollaire 4.2.2. - Soit $X \rightarrow X^{\bullet}$ un objet cosimplicial augmenté de h $\widehat{\mathcal{S}}$ tel que $X^{k}$ est sans $p$-torsion pour tout $k \geqslant 0$; alors les conditions suivantes sont équivalentes :

(i) L'objet simplicial augmenté $\mathrm{H}^{*} X^{\bullet} \rightarrow \mathrm{H}^{*} X$ est acyclique dans $\mathcal{E}$.

(ii) $X$ est sans $p$-torsion et la $\widehat{\mathrm{L}}$-algèbre simpliciale augmentée $\widehat{\mathrm{MU}}^{*} X \cdot \rightarrow \widehat{\mathrm{MU}}^{*} X$ est acyclique. 
Démonstration. - La $\widehat{\mathrm{L}}$-algèbre simpliciale $\widehat{\mathrm{MU}^{*}} X^{\bullet}$ est un objet simplicial de $\widehat{\mathcal{L}}$ et le morphisme $\left(\widehat{\mathrm{MU}}^{*} X^{\bullet}\right) / \mathrm{f}^{1} \rightarrow \mathrm{H}^{*} X^{\bullet}$ est un isomorphisme entre objets simpliciaux de $\mathcal{E}$ par la proposition 2.1.11.

Supposons la condition (i) vérifiée. Alors $X$ est sans $p$-torsion par le point (b) du lemme 2.1.7 donc $\widehat{\mathrm{MU}}^{*} X$ est dans $\widehat{\mathcal{L}}$ et l'application $\left(\widehat{\mathrm{MU}}^{*} X\right) / \mathrm{f}^{1} \rightarrow \mathrm{H}^{*} X$ est un isomorphisme par la proposition 2.1.11. Le point (b) de la proposition 4.2.1 montre que le morphisme $\pi_{0}\left(\widehat{\mathrm{MU}}^{*} X^{\bullet}\right) \rightarrow \widehat{\mathrm{MU}}^{*} X$ est un isomorphisme. Le point (a) de la même proposition montre que la $\widehat{\mathrm{L}}$-algèbre simpliciale augmentée $\widehat{\mathrm{MU}}^{*} X^{\bullet} \rightarrow \pi_{0}\left(\widehat{\mathrm{MU}}^{*} X^{\bullet}\right)$ est acyclique.

Inversement si la condition (ii) est vérifiée alors $\widehat{\mathrm{MU}}^{*} X$ est dans $\widehat{\mathcal{L}}$ et $\left(\widehat{\mathrm{MU}}^{*} X\right) / \mathrm{f}^{1} \rightarrow$ $\mathrm{H}^{*} X$ est un isomorphisme de sorte qu'on obtient (i) par le point (a) de la proposition 4.2.1.

La même discussion s'applique à la $\widetilde{G}$-résolution simpliciale d'une MU-algèbre instable non unitaire et à la MU-résolution cosimpliciale d'un espace profini pointé. Si $M$ est une MU-algèbre instable augmentée, le morphisme $\widetilde{\mathrm{G}}_{\bullet}(\widetilde{M})_{+} \rightarrow \widetilde{M}_{+}=M$ entre MU-algèbres instables augmentées simpliciales est une résolution de $M$ par des G-algèbres libres.

Cocomplexes de $\widehat{\mathcal{S}}_{\mathrm{pt}}$ et résolutions. - Les espaces profinis intervenant dans cette section sont le plus souvent pointés. Si $X$ est un espace profini quelconque, on peut toujours le remplacer par $X_{+}$, la réunion disjointe de $X$ et d'un point base.

Soit $X$ un espace profini pointé. On appelle MU-résolution libre de $X$ une suite de cofibrations $C^{n-1} \rightarrow X^{n}$ entre espaces profinis pointés vérifiant :

- on a $C^{-1}=X$ et pour tout entier $n \geqslant 0, C^{n}=X^{n} / C^{n-1}$;

- pour tout entier $n \geqslant 0$, le morphisme $\widetilde{\mathrm{MU}^{*}} X^{n} \rightarrow \widetilde{\mathrm{MU}^{*}} C^{n-1}$ est un épimorphisme;

- pour tout entier $n \geqslant 0$, l'espace profini $X^{n}$ est sans $p$-torsion.

La suite $X \rightarrow X^{*}$ formée de l'application $X \rightarrow X^{0}$ et des composées $X^{n} \rightarrow C^{n} \rightarrow$ $X^{n+1}$ est alors un cocomplexe augmenté de $\widehat{\mathcal{S}}_{\text {pt }}$ et induit en MU-cohomologie continue réduite une résolution libre de la $\widehat{\mathrm{L}}$-algèbre $\widehat{\mathrm{MU}}^{*} X$.

Observons que la suite de cofibrations $C^{n-1} \rightarrow X^{n}$ est déterminée par le cocomplexe augmenté $X \rightarrow X^{*}$ de $\widehat{\mathcal{S}}_{\text {pt }}$; on notera donc $X \rightarrow X^{*}$ une MU-résolution libre de $X$. Inversement on a le

Lemme 4.2.3. - Soit $X \rightarrow X^{*}$ un cocomplexe augmenté de $\widehat{\mathcal{S}}_{\mathrm{pt}}$, alors il existe une suite de cofibrations $C^{\prime n-1} \rightarrow X^{\prime n}$ et une suite de morphismes $X^{\prime n} \rightarrow X^{n}$ telles que :

$-C^{\prime-1}=X$ et $C^{\prime n}$ est le quotient de $X^{\prime n}$ par $C^{\prime n-1}$. 
- Les diagrammes
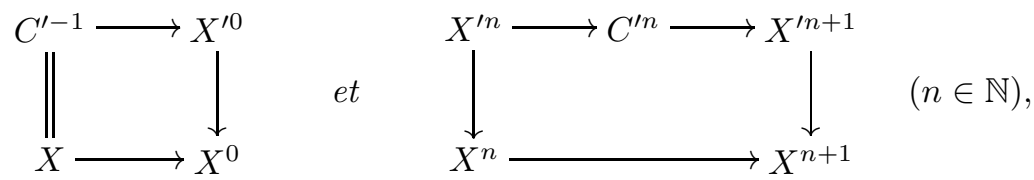

commutent.

- L'application $X^{\prime n} \rightarrow X^{n}$ est une équivalence faible pour tout entier $n$.

Démonstration. - On pose $C^{\prime-1}=X^{0}$ et on construit inductivement $X^{\prime n}$ comme l'objet cylindre $X^{n} \cup_{C^{\prime n-1}} C^{\prime n-1} \wedge \triangle[1]_{+}$et $C^{\prime n}$ comme le quotient de $X^{\prime n}$ par $C^{\prime n-1}$ avec la condition que la composée $C^{\prime n-1} \rightarrow X^{\prime n} \rightarrow X^{n} \rightarrow X^{n+1}$ est triviale (c'est vrai par hypothèse pour $n=0)$. L'application $X^{\prime n} \rightarrow X^{n}$ est alors une équivalence faible. La composée $X^{\prime n} \rightarrow X^{n} \rightarrow X^{n+1}$ se factorise par l'application $X^{\prime n} \rightarrow C^{\prime n}$ puisque sa restriction à $C^{\prime n-1}$ est triviale. La composée $X^{\prime n} \rightarrow X^{n} \rightarrow X^{n+1} \rightarrow X^{n+2}$ est triviale par hypothèse sur $X \rightarrow X^{*}$, donc également la composée $C^{\prime n} \rightarrow X^{n+1} \rightarrow X^{n+2}$ puisque l'application $X^{\prime n} \rightarrow C^{\prime n}$ est surjective degré par degré, ce qui permet de conclure.

Lemme 4.2.4. - Soit $X$ un espace profini pointé; alors $X$ admet une MU-résolution libre $X \rightarrow X^{*}$ telle que pour tout entier $n X^{n}$ est faiblement équivalent à l'image par $\mathrm{K}$ d'un ensemble gradué.

Démonstration. - On construit inductivement une telle résolution en posant $C^{-1}=$ $X$ et, si $C^{n-1}$ est déjà construit, en choisissant une cofibration $C^{n-1} \rightarrow X^{n}$ isomorphe dans h $\widehat{\mathcal{S}}_{\mathrm{pt}}$ à l'unité d'adjonction $C^{n-1} \rightarrow \mathrm{K}\left(\widetilde{\mathrm{MU}^{*}} C^{n-1}\right)$ et en posant $C^{n}=X^{n} / C^{n-1}$.

La proposition suivante montre qu'une telle résolution est universelle :

Proposition 4.2.5. - Soient $X \rightarrow X^{*}$ et $Y \rightarrow K^{*}$ des MU-résolutions libres d'espaces profinis pointés $X$ et $Y$. On suppose que pour tout entier $n K^{n}$ est fibrant et isomorphe dans $\mathrm{h} \widehat{\mathcal{S}}_{\mathrm{pt}}$ à l'image par $\mathrm{K}$ d'un ensemble gradué; alors toute application $X \rightarrow Y$ se relève en un morphisme de cocomplexes de $\widehat{\mathcal{S}}_{\mathrm{pt}}$ de $\left(X \rightarrow X^{*}\right)$ dans $\left(Y \rightarrow K^{*}\right)$ et deux tels relevés induisent en MU-cohomologie continue réduite des morphismes homotopes de complexes de $\widehat{\mathcal{M}}$.

Démonstration. - Notons $\left(C^{n-1} \rightarrow X^{n}\right)$ et $\left(D^{n-1} \rightarrow K^{n}\right)$ les suites de cofibrations dont proviennent $X \rightarrow X^{*}$ et $Y \rightarrow K^{*}$.

\section{Remarques}

- Prenons pour $\mathcal{L}$ la classe des objets en groupe abélien $\mathrm{K}(S)$ de h $\widehat{\mathcal{S}}_{\mathrm{pt}}, S$ décrivant les ensembles gradués. On vérifie d'une part qu'un cocomplexe augmenté de $\mathrm{h} \widehat{\mathcal{S}}_{\mathrm{pt}}$ est acyclique relativement à $\mathcal{L}$ si et seulement si le complexe augmenté induit en MU-cohomologie continue réduite est acyclique, d'autre part que deux morphismes 
entre cocomplexes de $\mathrm{h} \widehat{\mathcal{S}}_{\mathrm{pt}}$ sont homotopes relativement à $\mathcal{L}$ si et seulement si ils induisent en MU-cohomologie continue réduite des morphismes homotopes entre complexes de $\widehat{\mathcal{M}}$.

- Soit $X \rightarrow K^{*}$ un cocomplexe augmenté de h $\widehat{\mathcal{S}}_{\mathrm{pt}}$ induisant un complexe augmenté acyclique en MU-cohomologie réduite et tel que $K^{n}$ est dans $\mathcal{L}$ pour tout $n$. On peut montrer qu'il existe un cocomplexe augmenté $X \rightarrow K^{\prime *}$ de $\widehat{\mathcal{S}}_{\mathrm{pt}}$ isomorphe à $X \rightarrow K^{*}$ comme cocomplexe de $\mathrm{h} \widehat{\mathcal{S}}_{\mathrm{pt}}{ }^{(2)}$.

On dit qu'une MU-résolution libre $X \rightarrow X^{*}$ d'un espace profini pointé $X$ est de longueur $n$ si $X^{k}$ est le point pour tout $k>n$ et $n$ est minimal avec cette propriété. De façon équivalente, si $\left(C^{n-1} \rightarrow X^{n}\right)$ est la suite de cofibrations dont provient $X \rightarrow X^{*}$, la MU-résolution est de longueur $n$ si $C^{k}$ est le point pour tout $k \geqslant n$ et $n$ est minimal avec cette propriété.

EXEMPLE. - Soit $n$ un entier positif et soit $\eta$ le fibré complexe en droites canonique de base le classifiant de $\mathrm{S}^{1}$. La cofibre de l'application canonique $\mathrm{BZ} / p^{n} \rightarrow \mathrm{BS}^{1}$ s'identifie à l'espace de Thom, qu'on note $\mathrm{Th}_{n}$, du fibré $\eta^{\otimes p^{n}}$ (le produit tensoriel de $p^{n}$ copies de $\eta$ ). L'isomorphisme de Thom montre que l'espace $\mathrm{Th}_{n}$ est sans $p$-torsion de sorte que la suite $\mathrm{BZ} / p_{+}^{n} \rightarrow \mathrm{BS}_{+}^{1} \rightarrow \mathrm{Th}_{n} \rightarrow$ pt est une MU-résolution libre de $\mathrm{B} \mathbb{Z} / p_{+}^{n}$ de longueur 1 .

Proposition 4.2.6. - Soit $X$ un espace profini pointé de dimension finie, alors $X$ admet une MU-résolution libre de longueur finie par des espaces profinis de dimension finie.

Démonstration. - Supposons $X$ de dimension $n$. On construit d'abord inductivement une MU-résolution libre de $X$ par des espaces profinis de dimension inférieure ou égale à $n$. Posons $C^{-1}=X$; l'espace $C^{k-1}$ étant construit et de dimension inférieure ou égale à $n$, on choisit une cofibration $C^{k-1} \rightarrow Y$ isomorphe dans h $\widehat{\mathcal{S}}_{\mathrm{pt}}$ à l'unité d'adjonction $C^{k-1} \rightarrow \mathrm{K}\left(\widetilde{\widehat{\mathrm{MU}}^{*}} C^{k-1}\right)$. Cette cofibration se factorise par le squelette $n$-ième de $Y$ qui est encore sans $p$-torsion. On pose alors $X^{k}=\operatorname{Sk}_{n}(Y)$ et $C^{k}=$ $X^{k} / C^{k-1}$. L'espace profini $C^{k}$ est de dimension inférieure ou égale à $n$.

Le début de la résolution libre $\widetilde{\widehat{\mathrm{MU}}^{*}} X^{1} \rightarrow{\widetilde{\mathrm{MU}^{*}}}^{0} \rightarrow \widetilde{\widehat{\mathrm{MU}}^{*} X}$ montre que $\widetilde{\widehat{M U}}^{*} X$ est une $\widehat{\mathrm{L}}$-algèbre de présentation en degré borné. Elle admet une résolution libre de longueur finie, disons $m$, par la proposition 4.1.28. La $\widehat{\mathrm{L}}$-algèbre $\widehat{\widehat{\mathrm{MU}}}^{*} C^{m}=\operatorname{Ker}\left(\widehat{\widehat{\mathrm{MU}}}^{*} X^{m} \rightarrow \widehat{\widehat{\mathrm{MU}}}^{*} X^{m-1}\right)$ est alors dans $\widehat{\mathcal{L}}$ par la proposition 4.1 .8 de sorte que l'espace profini $C^{n}$ est sans $p$-torsion par la proposition 2.1.8. La suite $X \rightarrow X^{0} \rightarrow \cdots \rightarrow X^{m} \rightarrow C^{m} \rightarrow$ pt est une MU-résolution libre de $X$ de longueur $m+1$.

(2) Je remercie W.G. Dwyer de m'avoir dénoncé à ce propos un énoncé faux dans une première version de ce travail. 
4.3. MU-résolutions et suites spectrales. - Soit $M$ une théorie cohomologique réduite sur $\mathrm{h} \widehat{\mathcal{S}}_{\mathrm{pt}}$, i.e. un foncteur $M^{*}(-)$ défini sur $\mathrm{h} \widehat{\mathcal{S}}_{\mathrm{pt}}$, à valeurs dans la catégorie des groupes abéliens gradués, muni pour toute cofibration $X \rightarrow Y$ dans $\widehat{\mathcal{S}}_{\mathrm{pt}}$ d'un morphisme $M^{*} X \rightarrow M^{*+1} Y / X$ naturel en $X \rightarrow Y$ et induisant un triangle exact

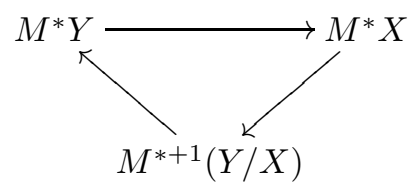

Soit $X \rightarrow X^{*}$ une MU-résolution d'un espace profini pointé $X$ et notons $\left(C^{n-1} \rightarrow X^{n}\right)$ la suite de cofibrations dont provient la résolution. On pose $A^{-s, *}=M^{*+s+1} C^{s}$ pour $-s \leqslant 1, A^{-s, *}=A^{1, *}=M^{*} X$ pour $-s>1$, et $E_{1}^{-s, *}=M^{*+s} X^{s}$. Le groupe abélien gradué $A^{1} \simeq M^{*} X$ a une filtration croissante naturelle $\left(\mathrm{F}_{s} A^{1}\right)_{s \geqslant-1}$ définie par $\mathrm{F}_{s} A^{1}=\operatorname{Ker}\left(A^{1} \rightarrow A^{-s}\right)$. Les cofibrations $C^{s-1} \rightarrow X^{s}, s \geqslant 0$ induisent un triangle exact

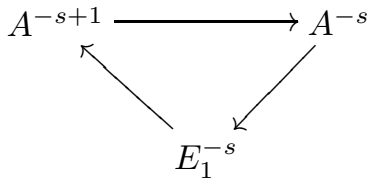

où $A^{-s, *} \rightarrow E_{1}^{-s, *}$ est un morphisme de bidegré $(0,1)$, donc une suite spectrale $\left(E_{r}, d_{r}: E_{r}^{-s, t} \rightarrow E_{r}^{-s+r, t+1}\right)$ vérifiant ( $c f$. les lemmes 5.6 et 5.9 et le théorème 6.1 de [Boa]) :

- Le terme $E_{1}^{s}$ est nul pour $s>0$ donc la suite $\left(E_{r}^{s}\right)_{r}$ est une suite d'épimorphismes pour $r>s$. On pose $E_{\infty}^{s}=\operatorname{colim}_{r} E_{r}^{s}$

- On a un épimorphisme $E_{\infty}^{-s} \rightarrow \mathrm{F}_{s} A^{1} / \mathrm{F}_{s-1} A^{1}$, et en particulier un morphisme de bord $E_{r}^{0} \rightarrow \mathrm{F}_{0} A^{1} \subset A^{1}$.

- Les conditions suivantes sont équivalentes :

(1) La filtration de $A^{1}$ est exhaustive et $E_{\infty}^{-s} \rightarrow \mathrm{F}_{s} A^{1} / \mathrm{F}_{s-1} A^{1}$ est un isomorphisme pour tout $s$

(2) le groupe abélien gradué $\operatorname{colim}_{s} A^{-s}$ est nul.

En particulier la suite spectrale converge fortement vers $A^{1}$ s'il existe un entier $s_{0}$ tel que $A^{-s}$ est nul pour tout $s \geqslant s_{0}$.

On réindexe la suite spectrale en posant $\mathrm{E}_{r}^{-s, t}=E_{r}^{-s, t+s}$ de sorte que le terme $\mathrm{E}_{1}$ est donné par $\mathrm{E}_{1}^{-s, t}=M^{t} X^{s}$ et la différentielle $\mathrm{d}_{r}$ est de bidegré $(r, 1-r)$. Le terme $\mathrm{E}_{2}^{-s, t}$ est l'homologie en degré $s$ du complexe $M^{t} X^{*}$ et on dispose d'un morphisme de bord $\mathrm{E}_{2}^{0, *} \rightarrow M^{*} X$

On suppose maintenant qu'on a un foncteur additif $M^{\prime}$ de la catégorie des $\widehat{\mathrm{L}}$ algèbres dans la catégorie des groupes abéliens gradués et, pour tout espace profini pointé $Y$, un morphisme naturel $M^{\prime}\left(\widetilde{\mathrm{MU}^{*}} Y\right) \rightarrow M^{*} Y$ qui est un isomorphisme si $Y$ 
est sans $p$-torsion. Le terme $\mathrm{E}_{2}^{-s, *}$ de la suite spectrale associée à $X \rightarrow X^{*}$ et $M$ est alors naturellement isomorphe à l'homologie en degré $s$ du complexe de $\widehat{L}$-algèbres $M^{\prime}\left(\widetilde{\widehat{\mathrm{MU}}}^{*} X^{*}\right)$, laquelle ne dépend que de la $\widehat{\mathrm{L}}$-algèbre $\widetilde{\widehat{\mathrm{MU}}}^{*} X$ à unique isomorphisme près.

Fixons une MU-résolution $X \rightarrow K^{*}$ de $X$ telle que pour tout $n K^{n}$ est de la forme $\mathrm{K}(S)$ pour un ensemble gradué $S$. Le point (b) de la proposition 4.2 .5 montre que l'identité de $X$ s'étend en un morphisme $\left(X \rightarrow X^{*}\right) \rightarrow\left(X \rightarrow K^{*}\right)$ entre cocomplexes augmentés de $\widehat{\mathcal{S}}_{\mathrm{pt}}$, lequel induit un morphisme entre les suites spectrales associées et une équivalence d'homotopie entre les complexes $\widetilde{\mathrm{MU}^{*}} K^{*}$ et $\widetilde{\mathrm{MU}^{*}} X^{*}$ donc un isomorphisme au niveau des termes $\mathrm{E}_{2}$ des suites spectrales.

Nous illustrons cette suite spectrale par deux exemples : les généralisations de la formule de Künneth et de la formule de coefficients universels ( $c f$. [Ad1] et $[\mathbf{C S}]$ pour le cas classique).

Pour le premier, soient $X$ et $Y$ deux espaces profinis pointés. On prend pour $M$ le foncteur $\widehat{\widehat{\mathrm{MU}}}^{*}(-\wedge Y)$. (Le foncteur $-\wedge Y$ commute aux cofibres homotopiques par construction.) A la théorie cohomologique $M$ et à une MU-résolution libre $X \rightarrow X^{*}$ de $X$ correspond une suite spectrale qui converge fortement vers $\widetilde{\widehat{M U}}^{*}(X \wedge Y)$ si la résolution $X \rightarrow X^{*}$ est de longueur finie. On dispose de plus d'un morphisme naturel

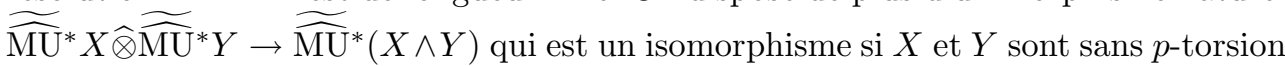
par la proposition 2.1.13.

Proposition 4.3.1. - Soient $X$ et $Y$ deux espaces profinis pointés. On suppose que $X$ admet une MU-résolution libre de longueur finie $X \rightarrow X^{*}$ telle que pour tout entier n l'application $\widetilde{\widehat{\mathrm{MU}}}^{*} X^{n} \widehat{\widehat{\otimes}} \widehat{\widehat{\mathrm{MU}}}^{*} Y \rightarrow \widetilde{\widehat{\mathrm{MU}}}^{*}\left(X^{n} \wedge Y\right)$ est un isomorphisme. Alors il existe une suite spectrale naturelle en $X$ et $Y$ convergeant fortement vers $\widehat{\mathrm{MU}}^{*}(X \wedge Y)$ et vérifiant :

(a) Le terme $\mathrm{E}_{2}^{-s, *}$ s'identifie à la $\widehat{\mathrm{L}}$-algèbre $\operatorname{Tor}_{s}\left(\widetilde{\widehat{\mathrm{MU}}^{*}} X, \widetilde{\widehat{\mathrm{MU}}}{ }^{*} Y\right)$ et le morphisme de bord ì ${\widetilde{\mathrm{MU}^{*}}}^{*} \widehat{\widehat{\otimes}}^{*} Y \rightarrow \widehat{\widehat{\mathrm{MU}}}^{*}(X \wedge Y)$.

(b) $S i \operatorname{Tor}_{s}\left(\widehat{\widehat{\mathrm{MU}}}^{*} X, \widehat{\widehat{\mathrm{MU}}}^{*} Y\right)$ est nul pour tout entier $s>0$ alors le morphisme de bord est un isomorphisme.

Démonstration. - Le point (a) de la proposition vient de ce que le complexe augmenté ${\widetilde{\mathrm{MU}^{*}}}^{*}\left(X^{*} \wedge Y\right) \rightarrow \widetilde{\widetilde{\mathrm{MU}^{*}}}(X \wedge Y)$ s'identifie à $\widetilde{\widehat{\mathrm{MU}}^{*}} X^{*} \widehat{\widehat{\otimes}}^{*} Y \rightarrow \widetilde{\widehat{\mathrm{MU}}}^{*}\left(X^{*} \wedge Y\right)$. Le point (b) vient de la convergence forte de la suite spectrale associée à $X^{*}$ et du fait que son terme $\mathrm{E}_{2}$ est concentré sur la première colonne par le point (a); autrement

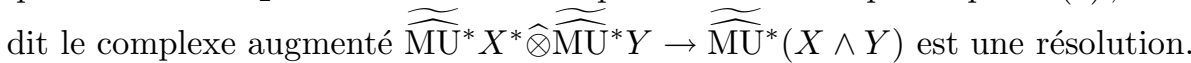

Proposition 4.3.2. - Soient $X$ et $Y$ deux espaces profinis pointés avec $Y$ sans $p$ torsion. On suppose de plus que l'une des conditions suivantes est satisfaite: 
(1) $X$ et $Y$ sont de dimension finie.

(2) La cohomologie modulo p continue de $Y$ est finie en chaque degré.

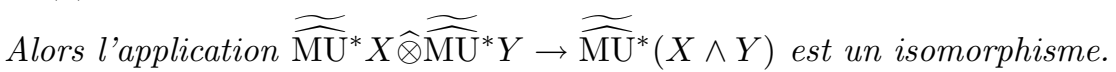

Démonstration. - Supposons $X$ de dimension finie. La proposition 4.2.6 nous dit qu'il existe un cocomplexe augmenté cofibrant de longueur finie $X \rightarrow X^{*}$ tel que

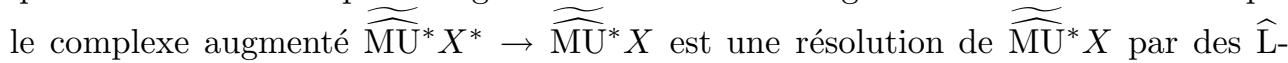
algèbres libres et nulles en degré assez grand. Sous l'une des conditions (1) ou (2) la $\widehat{\mathrm{L}}$-algèbre $\operatorname{Tor}_{s}\left(\widetilde{\widehat{\mathrm{MU}}}^{*} X, \widehat{\widehat{\mathrm{MU}}}^{*} Y\right)$ est nulle pour tout $s>0$ par le corollaire 4.1 .24 , d'où le résultat.

On obtient le cas $X$ quelconque sous la condition (2) en comparant les suites exactes

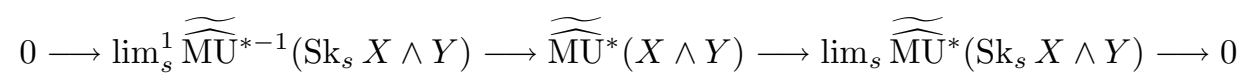

et

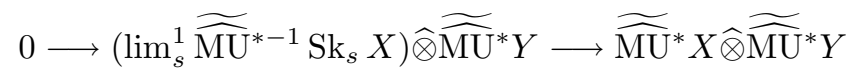

$$
\begin{aligned}
& \longrightarrow \lim _{s} \widetilde{\widehat{\mathrm{MU}}}^{*} \mathrm{Sk}_{s} X \widehat{\otimes} \widetilde{\widehat{\mathrm{MU}}^{*}} Y \longrightarrow 0,
\end{aligned}
$$

la seconde suite étant exacte par la proposition 4.1.24.

Remarque. - L'exactitude du foncteur $-\widehat{\otimes} \widehat{\widehat{M U}}^{*} Y$ sur $\widehat{\mathcal{M}}^{0}$ et la suite exacte de Mayer-Vietoris en MU-cohomologie continue permettent également de démontrer le corollaire.

Exemple. - Si $X$ et $Y$ sont de dimension finie ou si $X$ admet une MU-résolution libre de longueur finie $X \rightarrow X^{*}$ telle que pour tout entier $n$ la cohomologie modulo $p$ continue de $X^{n}$ est finie en chaque degré alors $X$ et $Y$ satisfont à l'hypothèse de la proposition 4.3.1 par la proposition 4.3.2.

On obtient des variantes «non pointées » de ces résultats en remplaçant $X$ et $Y$ par $X_{+}$et $Y_{+}$.

Prenons maintenant pour $M$ la cohomologie modulo $p$ continue réduite. On dispose d'un morphisme naturel $\left(\widehat{\widehat{\mathrm{MU}}^{*}} X\right) / \mathrm{f}^{1} \rightarrow \widetilde{\mathrm{H}}^{*} X$ qui est un isomorphisme si $X$ est sans $p$-torsion par la proposition 2.1.11. On obtient (nous donnons la version non pointée) :

Proposition 4.3.3. - Soit $X$ un espace profini admettant une MU-résolution libre de longueur finie; alors il existe une suite spectrale naturelle en $X$ de terme $\mathrm{E}_{2}^{-s, *}=$ $\operatorname{Tor}_{s}\left(\widehat{\mathrm{MU}}^{*} X, \mathrm{MU}^{*} / \mathrm{f}^{1}\right)$ convergeant fortement vers $\mathrm{H}^{*} X$ et dont le morphisme de bord s'identifie à $\left(\widehat{\mathrm{MU}}^{*} X\right) / \mathrm{f}^{1} \rightarrow \mathrm{H}^{*} X$.

L'hypothèse de la proposition est vérifiée si $X$ est de dimension finie. 


\section{Cohomologie des espaces fonctionnels et foncteurs de division}

Soit $W$ un ensemble simplicial dont la cohomologie modulo $p$ est finie en chaque degré; alors l'objet fonctionnel $X \mapsto \operatorname{hom}(W, X)$ se mime dans la catégorie des algèbres instables sur l'algèbre de Steenrod en l'adjoint à gauche du produit tensoriel par $\mathrm{H}^{*} W$. On appelle cet adjoint le foncteur de division par $\mathrm{H}^{*} W$ (voir [La1]).

La section 5.1 rappelle la construction des foncteurs de division dans les catégories $\mathcal{K}_{\mathrm{H}}$ et $\mathcal{K}_{\mathrm{H}-}$ à l'aide d'une division dans $\mathcal{E}$ et applique ces foncteurs de division à l'étude des espaces fonctionnels de but un espace d'Eilenberg-Mac Lane généralisé $\mathrm{K}_{\mathrm{H}}(E)$. On en déduit pour $W$ un ensemble simplicial quelconque et $X$ un espace profini une formule de Künneth pour la cohomologie modulo $p$ continue de l'espace profini $W \widehat{\times} X$ introduit dans la section 1.2 (lemme 5.1.3).

La section 5.2 montre l'existence des foncteurs de division $(-: P)$ associés à un objet $P$ dans les catégories $\mathcal{K}_{\mathrm{MU}}$ et $\mathcal{K}_{\mathrm{MU}}$ - lorsque la $\widehat{\mathrm{L}}$-algèbre sous-jacente à $P$ est libre et finie en chaque degré modulo $\mathrm{f}^{1}$. On obtient pour tout ensemble simplicial $W$ sans $p$-torsion dont la cohomologie modulo $p$ est finie en chaque degré et pour tout espace profini fibrant $X$ un morphisme

$$
\left(\widehat{\mathrm{MU}}^{*} X: \widehat{\mathrm{MU}}^{*} W\right)_{\mathcal{K}_{\mathrm{MU}}} \longrightarrow \widehat{\mathrm{MU}}^{*} \operatorname{hom}(W, X)
$$

qui est un isomorphisme si $X$ est l'image par K d'un ensemble gradué.

La dernière section (5.3) généralise l'étude algébrique des espaces profinis fonctionnels $\operatorname{hom}(W, X)$ aux $W$ quelconques (pour lesquels on n'a plus d'isomorphisme de Künneth décrivant la MU-cohomologie continue d'un produit mixte $W \widehat{\times} Z$ ) : On introduit le foncteur $\mathrm{T}_{W}$ qui associe à une MU-algèbre instable $M$ le coégalisateur dans $\mathcal{K}_{\mathrm{MU}}$ du 1-complexe $\widehat{\mathrm{MU}}^{*} \operatorname{hom}(W, \mathrm{~K}(\mathrm{G}(M))) \stackrel{\rightrightarrows}{\leftrightarrows} \widehat{\mathrm{MU}}^{*} \operatorname{hom}(W, \mathrm{~K}(M))$ induit par le 1-cocomplexe $\mathrm{K}(M) \leftrightarrows \mathrm{K}(\mathrm{G}(M))$ de $\mathrm{h} \widehat{\mathcal{S}}$. On dispose encore pour tout espace profini fibrant $X$ d'un morphisme

$$
\mathrm{T}_{W} \widehat{\mathrm{MU}}^{*} X \longrightarrow \widehat{\mathrm{MU}}^{*} \operatorname{hom}(W, X)
$$

qui, par construction, est un isomorphisme si $X$ est l'image par $\mathrm{K}$ d'un ensemble gradué. Nous démontrons quelques propriétés formelles de $\mathrm{T}_{W}$, notamment :

- $\mathrm{T}_{W}$ commute aux coégalisateurs de 1-complexes.

- On a une bijection naturelle

$$
\operatorname{Hom}_{\mathcal{K}_{\mathrm{MU}}}\left(M, \widehat{\mathrm{MU}}^{*} W\right) \stackrel{\sim}{\longrightarrow} \operatorname{Hom}_{\mathcal{K}_{\mathrm{MU}}}\left(\mathrm{T}_{W} M, \widehat{\mathrm{MU}}^{*}\right) .
$$

- Si $W$ est sans $p$-torsion et de cohomologie modulo $p$ finie en chaque degré alors on a un isomorphisme $T_{W} \cong\left(-: \widehat{\mathrm{MU}}^{*} W\right)_{\mathcal{K}_{\mathrm{MU}}}$.

5.1. Division dans $\mathcal{K}_{\mathrm{H}}$ - - Rappelons qu'à tout $\mathbb{F}_{p}$-espace vectoriel gradué $E$ correspond un espace profini pointé $\mathrm{K}_{\mathrm{H}}(E)$ dont la cohomologie modulo $p$ continue réduite est la $\widetilde{\mathrm{G}}_{\mathrm{H}}$-algèbre libre sur $E$, et un morphisme $E \rightarrow \widetilde{\mathrm{H}}^{*} \mathrm{~K}_{\mathrm{H}}(E)$ induisant des 
bijections naturelles

$$
\operatorname{Hom}_{\mathrm{h} \widehat{\mathcal{S}}_{\mathrm{pt}}}\left(X, \mathrm{~K}_{\mathrm{H}}(E)\right) \cong \operatorname{Hom}_{\mathcal{E}}\left(E, \widetilde{\mathrm{H}}^{*} X\right) \cong \operatorname{Hom}_{\mathcal{K}_{\mathrm{H}_{-}}}\left(\widetilde{\mathrm{H}}^{*} \mathrm{~K}_{\mathrm{H}}(E), \widetilde{\mathrm{H}}^{*} X\right)
$$

(voir la section 3). On fait les deux observations suivantes :

- Un morphisme $X \rightarrow \mathrm{K}_{\mathrm{H}}(E)$ dans $\mathrm{h} \widehat{\mathcal{S}}_{\mathrm{pt}}$ est un isomorphisme si et seulement s'il induit un isomorphisme en cohomologie modulo $p$ continue réduite (par définition de $\mathrm{h} \widehat{\mathcal{S}}_{\mathrm{pt}}$ ).

- Un morphisme $X \wedge Y \rightarrow \mathrm{K}_{\mathrm{H}}(E)$ dans h $\widehat{\mathcal{S}}_{\mathrm{pt}}$ induit grâce la formule de Künneth un morphisme $\widetilde{\mathrm{H}}^{*} \mathrm{~K}_{\mathrm{H}}(E) \rightarrow \widetilde{\mathrm{H}}^{*} X \otimes \widetilde{\mathrm{H}}^{*} Y$ dans $\mathcal{K}_{\mathrm{H}-}$.

On note $\mathcal{E}^{+}$la sous-catégorie pleine de $\mathcal{E}$ formée des $\mathbb{F}_{p}$-espaces vectoriels gradués nuls en degré strictement négatif. Pour $E \in \mathcal{E}$ et pour $s$ un entier on note $\mathrm{F}^{s} E$ le sous- $\mathbb{F}_{p}$-espace vectoriel gradué de $E$ formé des éléments de degré supérieur ou égal à $s$.

Lemme 5.1.1. - Soit $P$ un objet de $\mathcal{E}^{+}$de dimension finie en chaque degré; alors le foncteur $\mathcal{E}^{+} \rightarrow \mathcal{E}^{+}, E \mapsto P \otimes E$ admet un adjoint à gauche.

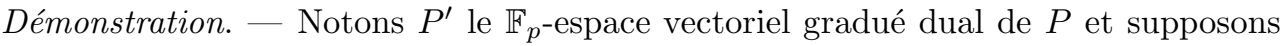
d'abord que $P$ est de dimension totale finie. Pour toute paire d'objets $E, F \in \mathcal{E}^{+}$ l'application $\operatorname{Hom}_{\mathcal{E}}\left(E, P^{\prime \prime} \otimes F\right) \rightarrow \operatorname{Hom}_{\mathcal{E}}\left(E \otimes P^{\prime}, F\right)$ est une bijection puisque $P^{\prime}$ est une somme finie de suspensions de $\mathbb{F}_{p}$. D'autre part $P$ est canoniquement isomorphe à son bidual $P^{\prime \prime}$. On en déduit que le foncteur $\mathcal{E}^{+} \rightarrow \mathcal{E}^{+}, E \mapsto \mathrm{F}^{0}\left(E \otimes P^{\prime}\right)$ est adjoint à gauche $\mathrm{du}$ foncteur $E \mapsto P \otimes E$.

Soit maintenant $P \in \mathcal{E}^{+}$de dimension finie en chaque degré; alors pour tout entier $s, P / \mathrm{F}^{s}$ est de dimension totale finie et les projections $P \rightarrow P / \mathrm{F}^{s}$ induisent pour tout objet $E \in \mathcal{E}^{+}$un isomorphisme $P \otimes E \rightarrow \lim _{s}\left(\left(P / \mathrm{F}^{s}\right) \otimes E\right)$. On en déduit que le foncteur $\mathcal{E}^{+} \rightarrow \mathcal{E}^{+}, E \mapsto \operatorname{colim}_{s} \mathrm{~F}^{0}\left(E \otimes\left(P / \mathrm{F}^{s}\right)^{\prime}\right) \cong \mathrm{F}^{0}\left(E \otimes P^{\prime}\right)$ est adjoint à gauche de $E \mapsto P \otimes E$.

On note $(E: P)$ l'objet $\mathrm{F}^{0}\left(E \otimes P^{\prime}\right)$. On déduit du lemme par adjonction :

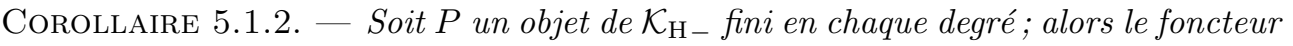
$\mathcal{K}_{\mathrm{H}_{-}} \rightarrow \mathcal{K}_{\mathrm{H}_{-}}, N \mapsto P \otimes N$ admet un adjoint à gauche qu'on note $(-: P)_{\mathcal{K}_{\mathrm{H}_{-}}}$.

Démonstration. - Soit $E$ un objet de $\mathcal{E}^{+}$. Le foncteur $N \mapsto \operatorname{Hom}_{\mathcal{K}_{\mathrm{H}_{-}}}\left(\widetilde{G}_{\mathrm{H}}(E), P \otimes N\right)$ est représentable par $\widetilde{G}_{\mathrm{H}}(E: P)$. Par universalité l'objet $\widetilde{G}_{\mathrm{H}}(E: P)$ est fonctoriel en $\widetilde{G}_{\mathrm{H}}(E) \in \mathcal{K}_{\mathrm{H}-}$.

Soit $M$ un objet de $\mathcal{K}_{\mathrm{H}_{-}}$; il est canoniquement le coégalisateur du 1-complexe $\left(\widetilde{\mathrm{G}}_{\mathrm{H}}\right)^{2}(M) \leftrightarrows \widetilde{\mathrm{G}}_{\mathrm{H}}(M)$. On définit $(M: P)_{\mathcal{K}_{\mathrm{H}_{-}}}$comme le coégalisateur du 1-complexe induit $\widetilde{\mathrm{G}}_{\mathrm{H}}\left(\widetilde{\mathrm{G}}_{\mathrm{H}}(M): P\right) \leftrightarrows \widetilde{\mathrm{G}}_{\mathrm{H}}(M: P)$; alors $(M: P)_{\mathcal{K}_{\mathrm{H}_{-}}}$représente le foncteur $M \mapsto \operatorname{Hom}_{\mathcal{K}_{\mathrm{H}_{-}}}(M, P \otimes N)$. 
Soient $W$ un ensemble fini simplicial pointé et $X$ un espace profini pointé. L'application d'évaluation $W \wedge \operatorname{hom}_{\mathrm{pt}}(W, X) \rightarrow X$ induit en cohomologie modulo $p$-continue réduite un morphisme $\widetilde{\mathrm{H}}^{*} X \rightarrow \widetilde{\mathrm{H}}^{*} W \otimes \widetilde{\mathrm{H}}^{*} \operatorname{hom}_{\mathrm{pt}}(W, X)$ donc un morphisme

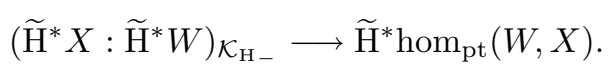

Soit $E$ un $\mathbb{F}_{p}$-espace vectoriel gradué nul en degré strictement négatif. Les bijections naturelles

$$
\begin{aligned}
& \operatorname{Hom}_{\mathrm{h} \widehat{\mathcal{S}}_{\mathrm{pt}}}\left(Z, \operatorname{hom}_{\mathrm{pt}}\left(W, \mathrm{~K}_{\mathrm{H}}(E)\right)\right) \cong \operatorname{Hom}_{\mathrm{h} \widehat{\mathcal{S}}_{\mathrm{pt}}}\left(W \wedge Z, \mathrm{~K}_{\mathrm{H}}(E)\right) \\
& \cong \operatorname{Hom}_{\mathrm{h} \widehat{\mathcal{S}}_{\mathrm{pt}}}\left(Z, \mathrm{~K}_{\mathrm{H}}\left(E: \widetilde{\mathrm{H}}^{*} W\right)\right)
\end{aligned}
$$

donnent un isomorphisme $\operatorname{hom}_{\mathrm{pt}}\left(W, \mathrm{~K}_{\mathrm{H}}(E)\right) \cong \mathrm{K}_{\mathrm{H}}\left(E: \widetilde{\mathrm{H}}^{*} W\right)$ dans $\mathrm{h} \widehat{\mathcal{S}}_{\mathrm{pt}}$ ou, de façon équivalente, montre que le morphisme

$$
\widetilde{\mathrm{H}}^{*} \mathrm{~K}_{\mathrm{H}}\left(E: \widetilde{\mathrm{H}}^{*} W\right) \cong\left(\widetilde{\mathrm{H}}^{*} \mathrm{~K}_{\mathrm{H}}(E): \widetilde{\mathrm{H}}^{*} W\right)_{\mathcal{K}_{\mathrm{H}_{-}}} \longrightarrow \widetilde{\mathrm{H}}^{*} \operatorname{hom}_{\mathrm{pt}}\left(W, \mathrm{~K}_{\mathrm{H}}(E)\right)
$$

est un isomorphisme dans $\mathcal{K}_{\mathrm{H}_{-}}$.

Soit maintenant $W$ un ensemble simplicial pointé quelconque qu'on écrit comme la colimite filtrante de ses sous-ensembles finis simpliciaux pointés $W_{\alpha}$. L'espace profini pointé $\operatorname{hom}_{\mathrm{pt}}(W, X)$ est par définition la limite filtrante des espaces profinis pointés $\operatorname{hom}_{\mathrm{pt}}\left(W_{\alpha}, X\right)$. Sa cohomologie modulo $p$ continue réduite est la colimite dans $\mathcal{E}$ comme dans $\mathcal{K}_{\mathrm{H}-}$ des cohomologies modulo $p$ continues réduites des $\operatorname{hom}_{\mathrm{pt}}\left(W_{\alpha}, X\right)$ par les lemmes 1.1 .1 et 3.2.2. On a donc un morphisme naturel

$$
\operatorname{colim}_{\alpha}\left(\widetilde{\mathrm{H}}^{*} X: \widetilde{\mathrm{H}}^{*} W_{\alpha}\right) \mathcal{K}_{\mathrm{H}_{-}} \longrightarrow \widetilde{\mathrm{H}}^{*} \operatorname{hom}_{\mathrm{pt}}(W, X)
$$

qu'on peut voir comme l'adjoint du morphisme

$$
\widetilde{\mathrm{H}}^{*} X \longrightarrow \lim _{\alpha}\left(\widetilde{\mathrm{H}}^{*} W_{\alpha} \otimes \widetilde{\mathrm{H}}^{*} \operatorname{hom}_{\mathrm{pt}}(W, X)\right) .
$$

Ce qui précède donne pour tout objet $E$ de $\mathcal{E}^{+}$un isomorphisme canonique $\operatorname{colim}_{\alpha} \widetilde{\mathrm{H}}^{*} \mathrm{~K}_{\mathrm{H}}\left(E: \widetilde{\mathrm{H}}^{*} W_{\alpha}\right) \cong \operatorname{colim}_{\alpha}\left(\widetilde{\mathrm{H}}^{*} \mathrm{~K}_{\mathrm{H}}(E): \widetilde{\mathrm{H}}^{*} W_{\alpha}\right)_{\mathcal{K}_{\mathrm{H}_{-}}} \stackrel{\sim}{\longrightarrow} \widetilde{\mathrm{H}}^{*} \operatorname{hom}_{\mathrm{pt}}\left(W, \mathrm{~K}_{\mathrm{H}}(E)\right)$ dans $\mathcal{K}_{\mathrm{H}-}$. Or la colimite dans $\mathcal{K}_{\mathrm{H}-\text { des }} \widetilde{\mathrm{G}}_{\mathrm{H}}$-algèbres libres sur les $\mathbb{F}_{p}$-espaces vectoriels gradués $\left(E: \widetilde{\mathrm{H}}^{*} W_{\alpha}\right)$ est par adjonction la $\widetilde{\mathrm{G}}_{\mathrm{H}}$-algèbre libre sur la colimite dans $\mathcal{E}$ des $\left(E: \widetilde{\mathrm{H}}^{*} W_{\alpha}\right)$. On obtient donc un isomorphisme

$$
\widetilde{\mathrm{H}}^{*} \mathrm{~K}_{\mathrm{H}}\left(\operatorname{colim}_{\alpha}\left(E: \widetilde{\mathrm{H}}^{*} W_{\alpha}\right)\right) \cong \widetilde{\mathrm{H}}^{*} \operatorname{hom}_{\mathrm{pt}}\left(W, \mathrm{~K}_{\mathrm{H}}(E)\right)
$$

dans $\mathcal{K}_{\mathrm{H}_{-}}$, c'est à dire un isomorphisme

$$
\operatorname{hom}_{\mathrm{pt}}\left(W, \mathrm{~K}_{\mathrm{H}}(E)\right) \cong \mathrm{K}_{\mathrm{H}}\left(\operatorname{colim}_{\alpha}\left(E: \widetilde{\mathrm{H}}^{*} W_{\alpha}\right)\right)
$$

dans $h \widehat{\mathcal{S}}_{\mathrm{pt}}$. En voici une application :

Lemme 5.1.3. - Soit $W$ un ensemble simplicial pointé qu'on écrit comme la colimite de ses sous-ensembles finis simpliciaux pointés $W_{\alpha}$ et soit $X$ un espace profini pointé. $L$ 'application $\widetilde{\mathrm{H}}^{*} W \widehat{\wedge} X \rightarrow \lim _{\alpha}\left(\widetilde{\mathrm{H}}^{*} W_{\alpha} \otimes \widetilde{\mathrm{H}}^{*} X\right)$ est un isomorphisme. 
(Rappelons que $W \widehat{\wedge} X$ désigne la colimite dans $\widehat{\mathcal{S}}_{\mathrm{pt}}$ des espaces profinis pointés $W_{\alpha} \wedge X$; voir la section 1.2 .)

Démonstration. — Il suffit de montrer que pour tout objet $E$ de $\mathcal{E}^{+}$l'application

$$
\begin{aligned}
& \operatorname{Hom}_{\mathcal{E}}\left(E, \widetilde{\mathrm{H}}^{*} W \widehat{\wedge} X\right) \longrightarrow \operatorname{Hom}_{\mathcal{E}}\left(E, \lim _{\alpha}\left(\widetilde{\mathrm{H}}^{*} W_{\alpha} \otimes \widetilde{\mathrm{H}}^{*} X\right)\right. \\
& \cong \operatorname{Hom}_{\mathcal{E}}\left(\operatorname{colim}_{\alpha}\left(E: \widetilde{\mathrm{H}}^{*} W_{\alpha}\right), \widetilde{\mathrm{H}}^{*} X\right)
\end{aligned}
$$

est une bijection ou, de façon équivalente, que l'application ci-dessus induit une bijection $\operatorname{Hom}_{\mathrm{h}} \widehat{\mathcal{S}}_{\mathrm{pt}}\left(W \widehat{\wedge} X, \mathrm{~K}_{\mathrm{H}}(E)\right) \cong \operatorname{Hom}_{\mathrm{h}} \widehat{\mathcal{S}}_{\mathrm{pt}}\left(X, \mathrm{~K}_{\mathrm{H}}\left(\operatorname{colim}_{\alpha}\left(E: \widetilde{\mathrm{H}}^{*} W_{\alpha}\right)\right)\right)$. Or ceci résulte

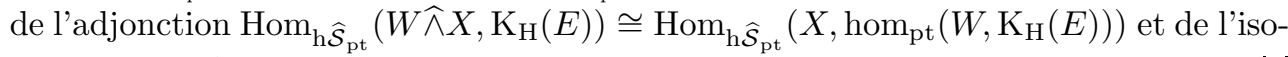
morphisme décrit avant le lemme.

Observons que le pro-objet $\left(\widetilde{\mathrm{H}}^{*} W_{\alpha}\right)_{\alpha}$ de $\mathcal{K}_{\mathrm{H}-}$ ne dépend (à pro-isomorphisme canonique près) que de la structure profinie de $\widetilde{\mathrm{H}}^{*} W$. On peut donc interpréter le morphisme $\widetilde{\mathrm{H}}^{*} X \rightarrow \lim _{\alpha}\left(\widetilde{\mathrm{H}}^{*} W_{\alpha} \otimes \widetilde{\mathrm{H}}^{*} \operatorname{hom}_{\mathrm{pt}}(W, X)\right)$ comme le morphisme induit en cohomologie modulo $p$ continue réduite par l'application d'évaluation $W \widehat{\wedge} \operatorname{hom}_{\mathrm{pt}}(W, X) \rightarrow X$.

COROLlaire 5.1.4. - Soient $W$ un ensemble simplicial pointé et $X$ un espace profini pointé; alors l'application $W \widehat{\wedge} X \rightarrow \widehat{W}^{f} \wedge X$ est une équivalence faible si et seulement si $\widetilde{\mathrm{H}}^{*} W$ ou $\widetilde{\mathrm{H}}^{*} X$ est fini en chaque degré.

Les résultats qui précèdent ont une version non pointée qui se démontre de la même façon et que nous nous contentons d'énoncer :

Soit $P$ une $\mathrm{HZ} / p$-algèbre instable fini en chaque degré; alors le foncteur $P \otimes-$ admet un adjoint à gauche $(-: P)_{\mathcal{K}_{\mathrm{H}}}$.

Soient $W$ un ensemble fini simplicial et $E$ un $\mathbb{F}_{p}$-espace vectoriel gradué nul en degré strictement négatif; alors l'application d'évaluation $W \times \operatorname{hom}\left(W, \mathrm{~K}_{\mathrm{H}}(E)\right) \rightarrow \mathrm{K}_{\mathrm{H}}(E)$ induit un isomorphisme d'algèbres instables

$$
\mathrm{H}^{*} \mathrm{~K}_{\mathrm{H}}\left(E: \mathrm{H}^{*} W\right) \cong\left(\mathrm{H}^{*} \mathrm{~K}_{\mathrm{H}}(E): \mathrm{H}^{*} W\right)_{\mathcal{K}_{\mathrm{H}}} \stackrel{\sim}{\longrightarrow} \mathrm{H}^{*} \operatorname{hom}\left(W, \mathrm{~K}_{\mathrm{H}}(E)\right) .
$$

Soit $W$ un ensemble simplicial qu'on écrit comme la colimite de ses sous-ensembles finis simpliciaux $W_{\alpha}$; alors on a un isomorphisme d'algèbres instables

$$
\mathrm{H}^{*} \mathrm{~K}_{\mathrm{H}}\left(\operatorname{colim}_{\alpha}\left(E: \mathrm{H}^{*} W_{\alpha}\right)\right) \cong \operatorname{colim}_{\alpha}\left(\mathrm{H}^{*} \mathrm{~K}_{\mathrm{H}}(E): \mathrm{H}^{*} W_{\alpha}\right)_{\mathcal{K}_{\mathrm{H}}} \stackrel{\sim}{\longrightarrow} \mathrm{H}^{*} \operatorname{hom}\left(W, \mathrm{~K}_{\mathrm{H}}(E)\right) \text {. }
$$

Les versions non pointées se déduisent en fait des versions pointées par adjonction :

- Soient $N$ une $\mathrm{HZ} / p$-algèbre instable augmentée, $P$ une $\mathrm{H} \mathbb{Z} / p$-algèbre instable finie en chaque degré et notons $\mathrm{O} P$ la $\mathrm{HZ} / p$-algèbre instable non unitaire sous-jacente à $P$. L'algèbre instable $(N: P)_{\mathcal{K}_{\mathrm{H}}}$ est augmentée par l'adjoint de la composée $N \rightarrow$ $\mathbb{F}_{p} \rightarrow P$ et comme telle s'identifie à $\left((\widetilde{N}: \mathrm{O} P)_{\mathcal{K}_{\mathrm{H}_{-}}}\right)_{+}=(\widetilde{N}: \mathrm{O} P)_{\mathcal{K}_{\mathrm{H}_{-}}} \oplus \mathbb{F}_{p}$. 
- Soient $W$ un ensemble fini simplicial et $X$ un espace profini pointé ; alors l'espace profini hom $(W, X)$ est pointé par l'application constante $W \rightarrow$ pt $\rightarrow X$, la cohomologie modulo $p$ réduite de $W_{+}$s'identifie à $\mathrm{OH}^{*} W$ et on a un diagramme commutatif

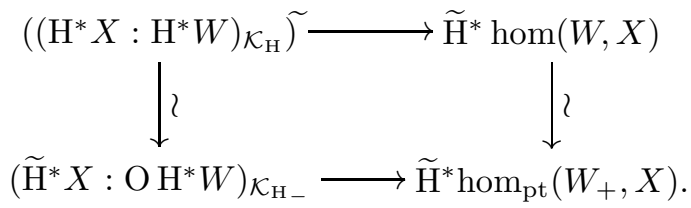

5.2. Division dans $\mathcal{K}_{\mathrm{MU}}$ • - On dispose pour tout objet $L \in \widehat{\mathcal{L}}$ d'un espace profini pointé $\mathrm{K}^{a}(L)$ et d'un morphisme $L \rightarrow \widehat{\widehat{\mathrm{MU}}}^{*} \mathrm{~K}^{a}(L)$ induisant pour tout espace profini pointé $X$ des bijections naturelles

$$
\operatorname{Hom}_{\mathrm{h} \widehat{\mathcal{S}}_{\mathrm{pt}}}\left(X, \mathrm{~K}^{a}(L)\right) \cong \operatorname{Hom}_{\widehat{\mathcal{M}}}\left(L, \widetilde{\widehat{\mathrm{MU}}^{*}} X\right) \cong \operatorname{Hom}_{\mathcal{K}_{\mathrm{MU}-}}\left(\widetilde{\widehat{\mathrm{MU}}^{*}} \mathrm{~K}^{a}(L), \widetilde{\widehat{\mathrm{MU}}^{*}} X\right) .
$$

On peut alors reproduire dans $\mathcal{K}_{\mathrm{MU}}$ l'analyse de la cohomologie continue des espaces fonctionnels mais il faut imposer des conditions sur l'ensemble simplicial pointé $W$ pour que, pour $X$ un espace profini pointé, l'objet $\widetilde{\mathrm{MU}^{*}} W \widehat{\wedge} X$ soit un foncteur en ${\widetilde{\mathrm{MU}^{*}}}^{*}$. Voici une condition suffisante :

LEMme 5.2.1. - Soit $W$ un ensemble simplicial pointé sans p-torsion et dont la cohomologie modulo $p$ est finie en chaque degré; alors on a pour tout espace profini pointé $X$ un isomorphisme naturel $\widehat{\widehat{\mathrm{MU}}}^{*} W \widehat{\otimes} \widehat{\widehat{\mathrm{MU}}}^{*} X \stackrel{\sim}{\longrightarrow} \widetilde{\widehat{\mathrm{MU}}}^{*} W \widehat{\wedge} X$.

Démonstration. - Observons d'abord que la complétion profinie donne un isomorphisme canonique $\widehat{\widehat{\mathrm{MU}}}^{*} W \cong \widehat{\widehat{\mathrm{MU}}}^{*} \widehat{W}^{f}$ (voir la section 1.6). Comme $\widehat{W}^{f}$ est sans $p$-torsion et de cohomologie modulo $p$ continue finie en chaque degré, la proposition 4.3.2 donne un isomorphisme $\widehat{\widehat{\mathrm{MU}}}^{*} W \widehat{\widehat{\otimes} \widehat{\mathrm{MU}}^{*}} X \rightarrow \widehat{\widehat{\mathrm{MU}}}^{*} \widehat{W}^{f} \wedge X$. Enfin le corollaire 5.1.4 montre que le morphisme $W \widehat{\wedge} X \rightarrow \widehat{W}^{f} \wedge X$ induit un isomorphisme en MUcohomologie continue réduite.

Lemme 5.2.2. - Soit $L$ un objet de $\widehat{\mathcal{L}}$ tel que $L / \mathrm{f}^{1}$ est fini en chaque degré.

(a) Le foncteur $\widehat{\mathcal{M}} \rightarrow \widehat{\mathcal{M}}, N \mapsto L \widehat{\otimes} N$ admet un adjoint à gauche qu'on note $(-: L)$

(b) Pour tout objet $M$ de $\widehat{\mathcal{L}}$ la $\widehat{\mathrm{L}}$-algèbre $(M: L)$ est dans $\widehat{\mathcal{L}}$.

Démonstration. - Choisissons un ensemble gradué $S$ et un isomorphisme $\widehat{\mathrm{L}}(S) \rightarrow L$; $S$ est fini en chaque degré. Pour $k$ entier positif notons $S_{k}$ l'ensemble gradué engendré par les éléments de degré compris entre $-k$ et $k$ et $S_{k}^{-}$l'ensemble gradué obtenu en changeant le signe des degrés des éléments de $S_{k}$. La $\widehat{\mathrm{L}}$-algèbre $\widehat{\mathrm{L}}\left(S_{k}\right)$ est un facteur direct de $\widehat{\mathrm{L}}(S)$ et $\widehat{\mathrm{L}}(S)$ est la limite dans $\widehat{\mathcal{M}}$ de la tour d'épimorphismes $\left(\widehat{\mathrm{L}}\left(S_{k}\right)\right)_{k}$. La $\widehat{\mathrm{L}}$-algèbre $\widehat{\mathrm{L}}\left(S_{k}^{-}\right)$s'identifie au $\widehat{\mathrm{MU}}^{*}$-module dual de $\widehat{\mathrm{L}}\left(S_{k}\right)$. 
Soit $M$ une $\widehat{\mathrm{L}}$-algèbre. Le foncteur $N \mapsto \operatorname{Hom}_{\widehat{\mathcal{M}}}\left(M, \widehat{\mathrm{L}}\left(S_{k}\right) \widehat{\otimes} N\right)$ est représentable par $M \widehat{\otimes} \widehat{\mathrm{L}}\left(S_{k}^{-}\right): \widehat{\mathrm{L}}\left(S_{k}\right) \widehat{\otimes} N$ est une somme finie de suspensions de $N$, voir l'appendice B. Par universalité la $\widehat{\mathrm{L}}$-algèbre $M \widehat{\otimes} \widehat{\mathrm{L}}\left(S_{k}^{-}\right)$est fonctorielle en $\widehat{\mathrm{L}}\left(S_{k}\right)$. La $\widehat{\mathrm{L}}$ algèbre $\widehat{\mathrm{L}}(S) \widehat{\otimes} N$ est la limite des $\widehat{\mathrm{L}}$-algèbres $\widehat{\mathrm{L}}\left(S_{k}\right) \widehat{\otimes} N$ par le lemme 4.1 .29 donc $N \mapsto \operatorname{Hom}_{\widehat{\mathcal{M}}}(M, \widehat{\mathrm{L}}(S) \widehat{\otimes} N)$ est représentable par la $\widehat{\mathrm{L}}$-algèbre $\operatorname{colim}_{k}\left(M \widehat{\otimes} \widehat{\mathrm{L}}\left(S_{k}^{-}\right)\right)$ d'où (a).

Si $M$ est dans $\widehat{\mathcal{L}}$ alors $M \widehat{\otimes} \widehat{\mathrm{L}}\left(S_{k}^{-}\right)$est dans $\widehat{\mathcal{L}}$ et $\operatorname{colim}_{k}\left(M \widehat{\otimes} \widehat{\mathrm{L}}\left(S_{k}^{-}\right)\right)$est dans $\widehat{\mathcal{L}}$ par le lemme 4.1.4 d'où (b).

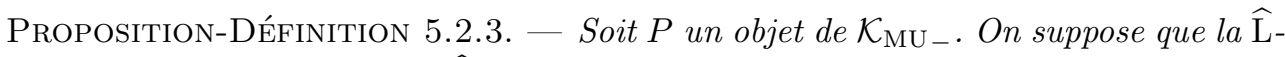
algèbre sous-jacente est dans $\widehat{\mathcal{L}}$ et que $P / \mathrm{f}^{1}$ est fini en chaque degré; alors le foncteur $\mathcal{K}_{\mathrm{MU}-} \rightarrow \mathcal{K}_{\mathrm{MU}_{-}}, N \mapsto P \widehat{\otimes} N$ admet un adjoint à gauche qu'on note $(-: P)_{\mathcal{K}_{\mathrm{MU}-}}$.

Démonstration. - Elle est identique à celle du corollaire 5.1.2 : pour toute $\widehat{\mathrm{L}}$-algèbre $M$ le foncteur $N \mapsto \operatorname{Hom}_{\mathcal{K}_{\mathrm{MU}-}}\left(\widetilde{\mathrm{G}}^{a}(M), P \widehat{\otimes} N\right)$ est représentable par $\widetilde{\mathrm{G}}^{a}(M: P)$ puis, pour tout objet $M$ de $\mathcal{K}_{\mathrm{MU}_{-}}$, le foncteur $N \mapsto \operatorname{Hom}_{\mathcal{K}_{\mathrm{MU}-}}(M, P \widehat{\otimes} N)$ est représentable par le coégalisateur du 1-complexe $\widetilde{\mathrm{G}}^{a}\left(\widetilde{\mathrm{G}}^{a}(M): P\right) \leftrightarrows \widetilde{\mathrm{G}}^{a}(M: P)$ induit par le 1complexe $\widetilde{\mathrm{G}}^{a 2}(M) \stackrel{\leftrightarrows}{\leftrightarrows} \widetilde{\mathrm{G}}^{a}(M)$ de $\mathcal{K}_{\mathrm{MU}-}$.

Soit $W$ un ensemble simplicial pointé sans $p$-torsion dont la cohomologie modulo $p$ est finie en chaque degré et $X$ un espace profini pointé. L'application d'évaluation $W \widehat{\wedge} \operatorname{hom}_{\mathrm{pt}}(W, X) \rightarrow X$ induit en MU-cohomologie continue réduite un morphisme $\widetilde{\widehat{\mathrm{MU}}^{*}} X \rightarrow \widetilde{\widehat{\mathrm{MU}}^{*}} W \widehat{\otimes} \widetilde{\mathrm{MU}^{*}} h^{2} \operatorname{pm}_{\mathrm{pt}}(W, X)$ par le lemme 5.2.1 donc un morphisme

$$
\left(\widetilde{\widehat{\mathrm{MU}}}^{*} X:{\widetilde{\mathrm{MU}^{*}}}^{*}\right)_{\mathcal{K}_{\mathrm{MU}-}} \longrightarrow \widetilde{\widehat{\mathrm{MU}}}^{*} \operatorname{hom}_{\mathrm{pt}}(W, X)
$$

dans $\mathcal{K}_{\mathrm{MU}-}$.

Soit $L$ un objet de $\widehat{\mathcal{L}}$. La $\widehat{\mathrm{L}}$-algèbre $\left(L: \widetilde{\widehat{\mathrm{MU}}}^{*} W\right)$ est dans $\widehat{\mathcal{L}}$ par le lemme 5.2.2. Les bijections naturelles

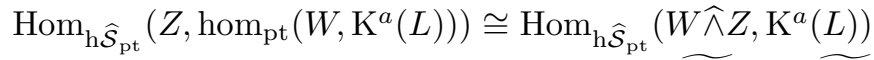

$$
\begin{aligned}
& \cong \operatorname{Hom}_{\widehat{\mathcal{M}}}\left(L, \widetilde{\widehat{\mathrm{MU}^{\prime}}} * \widehat{\widehat{\otimes}} \widehat{\widehat{\mathrm{MU}}}^{*} Z\right) \\
& \cong \operatorname{Hom}_{\widehat{\mathcal{M}}}\left(\left(L: \widetilde{\widehat{\mathrm{MU}}}^{*} W\right), \widetilde{\left.\widehat{\mathrm{MU}}^{*} Z\right)}\right. \\
& \cong \operatorname{Hom}_{\mathrm{h} \widehat{\mathrm{S}}_{\mathrm{pt}}}\left(Z, \mathrm{~K}^{a}\left(L: \widehat{\widehat{\mathrm{MU}}}^{*} W\right)\right)
\end{aligned}
$$

donnent un isomorphisme $\operatorname{hom}_{\mathrm{pt}}\left(W, \mathrm{~K}^{a}(L)\right) \cong \mathrm{K}^{a}\left(L: \widetilde{\widehat{\mathrm{MU}}}^{*} W\right)$ dans $\mathrm{h} \widehat{\mathcal{S}}_{\mathrm{pt}}$, donc montrent que l'espace profini hom $\operatorname{pt}_{\mathrm{pt}}\left(W, \mathrm{~K}^{a}(L)\right)$ est sans $p$-torsion et que le morphisme

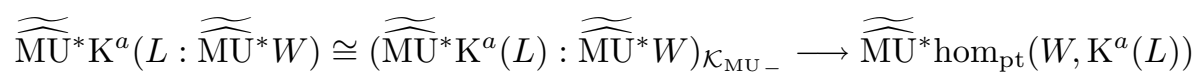

est un isomorphisme dans $\mathcal{K}_{\mathrm{MU}-\text {. }}$

Les résultats qui précèdent ont une version non pointée qui s'obtient de la même façon. Pour $P$ dans $\mathcal{K}_{\mathrm{MU}} \cap \widehat{\mathcal{L}}$ tel que $P / \mathrm{f}^{1}$ est fini en chaque degré, on note $(-: P)_{\mathcal{K}_{\mathrm{MU}}}$ 
l'adjoint à gauche de $P \widehat{\otimes}-$. Si $W$ est un ensemble simplicial sans $p$-torsion dont la cohomologie modulo $p$ est finie en chaque degré alors, pour tout espace profini pointé $X$, l'application d'évaluation $X \widehat{\times} \operatorname{hom}(W, X) \rightarrow X$ induit un morphisme

$$
\left(\widehat{\mathrm{MU}}^{*} X: \widehat{\mathrm{MU}}^{*} W\right)_{\mathcal{K}_{\mathrm{MU}}} \longrightarrow \widehat{\mathrm{MU}}^{*} \operatorname{hom}(W, X)
$$

qui est un isomorphisme si $X$ est l'image par $\mathrm{K}$ d'un ensemble gradué.

Notons O $P$ l'image de $P$ par l'oubli $\mathcal{K}_{\mathrm{MU}} \rightarrow \mathcal{K}_{\mathrm{MU}_{-}}$. Si $N$ est une MU-algèbre instable augmentée alors l'algèbre instable $(N: P)_{\mathcal{K}_{\mathrm{MU}}}$ est augmentée par l'adjoint de la composée $N \rightarrow \widehat{\mathrm{MU}}^{*} \rightarrow P$ et comme telle s'identifie à $\left((\widetilde{N}: \mathrm{O} P)_{\mathcal{K}_{\mathrm{MU}-}}\right)_{+}$. Si $X$ est un espace profini pointé alors hom $(W, X)$ est pointé par l'application constante et on a un diagramme commutatif

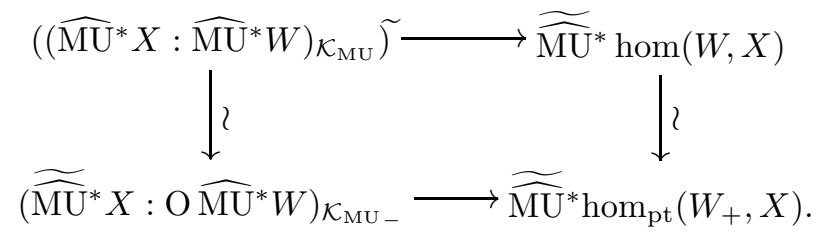

Observons enfin que pour $N$ dans $\mathcal{K}_{\mathrm{MU}-}$ l'unité d'adjonction $N \rightarrow P \widehat{\otimes}(N: P)_{\mathcal{K}_{\mathrm{MU}-}}$ induit un morphisme $N / \mathrm{f}^{1} \rightarrow P / \mathrm{f}^{1} \otimes(N: P)_{\mathcal{K}_{\mathrm{MU}-}} / \mathrm{f}^{1}(c f$. la discussion sur le produit tensoriel dans la section 3 ) donc un morphisme

$$
\left(N / \mathrm{f}^{1}: P / \mathrm{f}^{1}\right)_{\mathcal{K}_{-}} \longrightarrow(N: P)_{\mathcal{K}_{\mathrm{MU}-}} / \mathrm{f}^{1} .
$$

Ce morphisme a très peu de chance d'être un isomorphisme.

Généralisation. - Soit $\left(W_{\alpha}\right)$ un diagramme filtrant d'ensembles simpliciaux pointés sans $p$-torsion et de cohomologie modulo $p$ finie en chaque degré, dont on note $W$ la colimite, et soit $L$ un objet de $\widehat{\mathcal{L}}$. Pour chaque $\alpha$ la $\widehat{\mathrm{L}}$-algèbre $\left(L: \widetilde{\widehat{\mathrm{MU}}}^{*} W_{\alpha}\right)$ est dans $\widehat{\mathcal{L}}$ et on a un isomorphisme naturel $\mathrm{G}^{a}\left(L: \widetilde{\widehat{\mathrm{MU}}}^{*} W_{\alpha}\right) \cong \widetilde{\mathrm{MU}^{*}} \operatorname{hom}_{\mathrm{pt}}\left(W_{\alpha}, \mathrm{K}^{a}(L)\right)$ dans $\mathcal{K}_{\mathrm{MU}-}$.

L'espace profini pointé $\operatorname{hom}_{\mathrm{pt}}\left(W, \mathrm{~K}^{a}(L)\right)$ est par construction la limite des espaces profinis pointés $\operatorname{hom}_{\mathrm{pt}}\left(W_{\alpha}, \mathrm{K}^{a}(L)\right)$ donc est sans $p$-torsion par le lemme 2.1.15 et sa MU-cohomologie réduite est la colimite des objets $\widetilde{\widehat{M U}}^{*} h_{\mathrm{pt}}\left(W_{\alpha}, \mathrm{K}^{a}(L)\right)$ dans $\widehat{\mathcal{M}}$

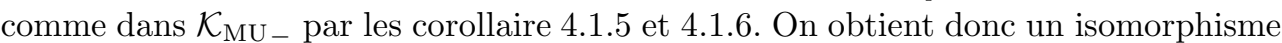

$$
\mathrm{G}^{a}\left(\operatorname{colim}_{\alpha}\left(L: \widetilde{\widehat{\mathrm{MU}}^{*}} W_{\alpha}\right)\right) \cong \widetilde{\widehat{\mathrm{MU}}} * \operatorname{hom}_{\mathrm{pt}}\left(W, \mathrm{~K}^{a}(L)\right)
$$

dans $\mathcal{K}_{\mathrm{MU}-}$, d'où un isomorphisme $\operatorname{hom}_{\mathrm{pt}}\left(W, \mathrm{~K}^{a}(L)\right) \cong \mathrm{K}^{a}\left(\operatorname{colim}_{\alpha}\left(L: \widetilde{\widehat{\mathrm{MU}}}^{*} W_{\alpha}\right)\right)$ dans h $\widehat{\mathcal{S}}_{\text {pt }}$ par le lemme 3.1.2

Soit $X$ un espace profini. La bijection naturelle

$$
\operatorname{Hom}_{\mathrm{h} \widehat{\mathcal{S}}_{\mathrm{pt}}}\left(X, \operatorname{hom}_{\mathrm{pt}}\left(W, \mathrm{~K}^{a}(L)\right)\right) \cong \operatorname{Hom}_{\mathrm{h} \widehat{\mathcal{S}}_{\mathrm{pt}}}\left(W \widehat{\wedge} X, \mathrm{~K}^{a}(L)\right)
$$


montre alors que le morphisme $\widetilde{\widetilde{\mathrm{MU}^{*}}} W \widehat{\wedge} X \rightarrow \lim _{\alpha}\left(\widetilde{\widehat{\mathrm{MU}}^{*}} W_{\alpha} \widehat{\widetilde{\otimes}}^{*} X\right)$ est un isomorphisme dans $\widehat{\mathcal{M}}$ (donc dans $\mathcal{K}_{\mathrm{MU}_{-}}$), de sorte qu'on peut interpréter l'isomorphisme

$$
\begin{aligned}
& \operatorname{colim}_{\alpha}\left(\mathrm{G}^{a}(L): \widetilde{\widehat{\mathrm{MU}}}^{*} W_{\alpha}\right)_{\mathcal{K}_{\mathrm{MU}-}} \cong \mathrm{G}^{a}\left(\operatorname{colim}_{\alpha}\left(L: \widetilde{\widetilde{\mathrm{MU}}^{*}} W_{\alpha}\right)\right)
\end{aligned}
$$

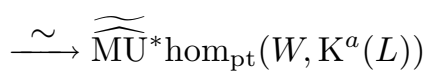

comme l'adjoint du morphisme de $\mathcal{K}_{\mathrm{MU}_{-}}$induit par l'application d'évaluation

$$
W \widehat{\wedge} \operatorname{hom}_{\mathrm{pt}}\left(W, \mathrm{~K}^{a}(L)\right) \longrightarrow \mathrm{K}^{a}(L) .
$$

5.3. Foncteur $\mathrm{T}_{W}$ - - Rappelons qu'à toute $\mathrm{MU}$-algèbre instable $M$ correspond

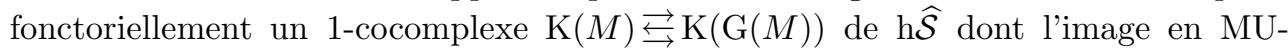
cohomologie continue est le début de la G-résolution simpliciale de $M$.

Soit $W$ un ensemble simplicial quelconque. On définit un foncteur $\mathrm{T}_{W}: \mathcal{K}_{\mathrm{MU}} \rightarrow$ $\mathcal{K}_{\mathrm{MU}}$ associant à un objet $M$ le coégalisateur dans $\mathcal{K}_{\mathrm{MU}}$ du 1-complexe

$$
\widehat{\mathrm{MU}}^{*} \operatorname{hom}(W, \mathrm{~K}(\mathrm{G}(M))) \rightleftarrows \widehat{\mathrm{MU}}^{*} \operatorname{hom}(W, \mathrm{~K}(M))
$$

(le foncteur $X \mapsto \operatorname{hom}(W, X)$ commute aux équivalences faibles entre objets fibrants).

Soit $X$ un espace profini fibrant. L'unité $X \rightarrow \mathrm{K}\left(\widehat{\mathrm{MU}}^{*} X\right)$ dans h $\widehat{\mathcal{S}}$ induit un morphisme

$$
\mathrm{T}_{W}\left(\widehat{\mathrm{MU}}^{*} X\right) \longrightarrow \widehat{\mathrm{MU}}^{*} \operatorname{hom}(W, X) .
$$

On vérifie d'une part que ce morphisme est un isomorphisme lorsque $X$ est l'image par K d'un ensemble gradué, d'autre part que l'image par $\mathrm{T}_{W}$ du diagramme

$$
\mathrm{G}^{2}(M) \rightleftarrows \mathrm{G}(M) \longrightarrow M
$$

associé à un objet $M$ de $\mathcal{K}_{\mathrm{MU}}$ est un diagramme coégalisateur dans $\mathcal{K}_{\mathrm{MU}}$ (voir la proposition A.2.2 et sa démonstration). En particulier l'identité $\widehat{\mathrm{MU}}^{*}=\mathrm{G}(\varnothing)$ donne un isomorphisme canonique

$$
\mathrm{T}_{W} \widehat{\mathrm{MU}}^{*} \cong \widehat{\mathrm{MU}}^{*}
$$

Proposition 5.3.1. — Soit $W$ un ensemble simplicial.

(a) Le foncteur $\mathrm{T}_{W}$ commute aux coégalisateurs de 1-complexes.

(b) Supposons que pour tout ensemble gradué $S$ l'espace profini hom $(W, \mathrm{~K}(S))$ est sans p-torsion; alors $\mathrm{T}_{W}$ commute aux colimites indexées par une catégorie petite.

(c) Supposons que $W$ est sans p-torsion et de cohomologie modulo p finie en chaque degré; alors on a pour toute MU-algèbre instable $M$ un isomorphisme canonique

$$
\left(M: \widehat{\mathrm{MU}}^{*} W\right)_{\mathcal{K}_{\mathrm{MU}}} \cong \mathrm{T}_{W} M .
$$

(d) Soit $W \rightarrow W^{\prime}$ une application entre ensembles simpliciaux induisant un isomorphisme en cohomologie modulo $p$; alors la transformation naturelle $\mathrm{T}_{W} \rightarrow \mathrm{T}_{W^{\prime}}$ est un isomorphisme. 
Démonstration. - Puisque la monade $\mathrm{G}$ vérifie la condition $\left(\mathcal{Q}_{0}\right)$, le point (a) vient du point (a) de la proposition A.1.6.

Pour le point (b) il suffit par cette même proposition de vérifier que le foncteur $\mathcal{E}$ ns-gr $\rightarrow \mathcal{K}_{\mathrm{MU}}, S \mapsto \mathrm{T}_{W} \mathrm{G}(S)$ commute aux sommes finies et aux colimites filtrantes. Or le foncteur $\mathcal{E}$ ns-gr $\rightarrow \widehat{\mathcal{S}}, S \mapsto \operatorname{hom}(W, \mathrm{~K}(S))$ transforme les colimites en limites. Comme pour tout ensemble gradué $S$ l'espace profini hom $(W, \mathrm{~K}(S))$ est sans $p$-torsion, l'image par $S \mapsto \widehat{\mathrm{MU}}^{*} \operatorname{hom}(W, \mathrm{~K}(S))$ de la somme de deux ensembles gradués est la somme des images dans $\mathcal{K}_{\mathrm{MU}}$ par la formule de Künneth et le point (b) de la proposition 3.1.4, donc $\mathrm{T}_{W}$ commute aux sommes finies. De même l'image d'une colimite filtrante d'ensembles gradués est la colimites filtrantes des images dans $\mathcal{K}_{\mathrm{MU}}$ par le corollaire 4.1.5, d'où le résultat.

Sous l'hypothèse du point (c) on a un isomorphisme canonique

$$
\left(\mathrm{G}(S): \widehat{\mathrm{MU}}^{*} W\right)_{\mathcal{K}_{\mathrm{MU}}} \cong \mathrm{T}_{W} \mathrm{G}(S)
$$

puisque tous deux sont canoniquement isomorphes à $\widehat{\mathrm{MU}}^{*} \operatorname{hom}(W, \mathrm{~K}(S))$. On en déduit alors pour toute MU-algèbre instable $M$, en utilisant le début de sa G-résolution simpliciale, un isomorphisme canonique $\left(M: \widehat{\mathrm{MU}}^{*} W\right)_{\mathcal{K}_{\mathrm{MU}}} \cong \mathrm{T}_{W} M$.

Soit enfin $W \rightarrow W^{\prime}$ une application induisant un isomorphisme en cohomologie modulo $p$; alors pour tout espace profini fibrant $X$ l'application $\operatorname{hom}\left(W^{\prime}, X\right) \rightarrow$ $\operatorname{hom}(W, X)$ est une équivalence faible de $\widehat{\mathcal{S}}(c f$. le début de la section 1.3) On en déduit que pour tout ensemble gradué $S$ le morphisme $\mathrm{T}_{W} \mathrm{G}(S) \rightarrow \mathrm{T}_{W^{\prime}} \mathrm{G}(S)$ est un isomorphisme de $\mathcal{K}_{\mathrm{MU}}$. On conclut comme pour le point (c) en utilisant le début de la G-résolution simpliciale d'un objet de $\mathcal{K}_{\mathrm{MU}}$.

Soit $X$ un espace profini fibrant dont on note $|X|$ l'ensemble simplicial sous-jacent. On dispose de bijections naturelles

$$
\operatorname{Hom}_{\mathrm{h} \widehat{\mathcal{S}}}\left(\widehat{W}^{f}, X\right) \cong \operatorname{Hom}_{\mathrm{h} \mathcal{S}}(W,|X|) \cong \pi_{0} \operatorname{hom}(W, X) .
$$

L'application

$$
\pi_{0} \operatorname{hom}(W, X) \longrightarrow \operatorname{Hom}_{\mathcal{K}_{\mathrm{MU}}}\left(\widehat{\mathrm{MU}}^{*} \operatorname{hom}(W, X), \widehat{\mathrm{MU}}^{*}\right)
$$

est une bijection par la proposition 3.3.3. En prenant $X=\mathrm{K}(S)$ on obtient une bijection $\operatorname{Hom}_{\mathcal{K}_{\mathrm{MU}}}\left(\mathrm{G}(S), \widehat{\mathrm{MU}}^{*} W\right) \stackrel{\sim}{\longrightarrow} \operatorname{Hom}_{\mathcal{K}_{\mathrm{MU}}}\left(\mathrm{T}_{W} \mathrm{G}(S), \widehat{\mathrm{MU}}^{*}\right)$ naturelle en $\mathrm{G}(S) \in$ $\mathcal{K}_{\mathrm{MU}}$. On en déduit pour tout objet $M \in \mathcal{K}_{\mathrm{MU}}$ en utilisant le début de la G-résolution simpliciale de $M$ une bijection naturelle

$$
\operatorname{Hom}_{\mathcal{K}_{\mathrm{MU}}}\left(M, \widehat{\mathrm{MU}}^{*} W\right) \stackrel{\sim}{\longrightarrow} \operatorname{Hom}_{\mathcal{K}_{\mathrm{MU}}}\left(\mathrm{T}_{W} M, \widehat{\mathrm{MU}}^{*}\right) .
$$

On en déduit à nouveau avec la proposition 3.3.3 la :

Proposition 5.3.2. - Soit $W$ un ensemble simplicial et $X$ un espace profini fibrant. Si le morphisme $\mathrm{T}_{W} \widehat{\mathrm{MU}}^{*} X \rightarrow \widehat{\mathrm{MU}}^{*} \operatorname{hom}(W, X)$ est un isomorphisme, alors l'application

$$
\operatorname{Hom}_{\mathrm{h} \mathcal{S}}(W,|X|) \longrightarrow \operatorname{Hom}_{\mathcal{K}_{\mathrm{MU}}}\left(\widehat{\mathrm{MU}}^{*} X, \widehat{\mathrm{MU}}^{*} W\right)
$$

est une bijection. 
Soient $W, W^{\prime}$ deux ensembles simpliciaux; l'isomorphisme

$$
\operatorname{hom}\left(W, \operatorname{hom}\left(W^{\prime}, \mathrm{K}(S)\right)\right) \cong \operatorname{hom}\left(W \times W^{\prime}, \mathrm{K}(S)\right)
$$

donne un morphisme $\mathrm{T}_{W} \mathrm{~T}_{W^{\prime}} \rightarrow \mathrm{T}_{W \times W^{\prime}}$.

\section{Lemme 5.3.3}

(a) Soit $\left(W_{\alpha}\right)$ un diagramme filtrant d'ensembles simpliciaux. On suppose que pour tout indice $\alpha$ et pour tout ensemble gradué $S$ l'espace profini $\operatorname{hom}\left(W_{\alpha}, \mathrm{K}(S)\right)$ est sans p-torsion; alors pour tout objet $M \in \mathcal{K}_{\mathrm{MU}}$ le morphisme

$$
\operatorname{colim}_{\alpha} \mathrm{T}_{W_{\alpha}} M \longrightarrow \mathrm{T}_{\operatorname{colim}_{\alpha} W_{\alpha}} M
$$

est un isomorphisme dans $\widehat{\mathcal{M}}$.

(b) Soient $W, W^{\prime}$ deux ensembles simpliciaux. Supposons que pour tout ensemble gradué $S$ le morphisme

$$
\mathrm{T}_{W} \widehat{\mathrm{MU}}^{*} \operatorname{hom}\left(W^{\prime}, \mathrm{K}(S)\right) \longrightarrow \widehat{\mathrm{MU}}^{*} \operatorname{hom}\left(W, \operatorname{hom}\left(W^{\prime}, \mathrm{K}(S)\right)\right)
$$

est un isomorphisme; alors le morphisme

$$
\mathrm{T}_{W} \mathrm{~T}_{W^{\prime}} \longrightarrow \mathrm{T}_{W \times W^{\prime}}
$$

est un isomorphisme.

Démonstration. - Il suffit pour le point (a) de montrer que pour tout ensemble gradué $S$ le morphisme colim ${ }_{\alpha} \mathrm{T}_{W_{\alpha}} \mathrm{G}(S) \rightarrow \mathrm{T}_{\text {colim }_{\alpha} W_{\alpha}} \mathrm{G}(S)$ est un isomorphisme. Or cela résulte de l'interprétation des objets comme MU-cohomologie d'espaces fonctionnels et du corollaire 4.1.5,

De même l'isomorphisme $\mathrm{T}_{W} \mathrm{~T}_{W^{\prime}} M \rightarrow \mathrm{T}_{W \times W^{\prime}} M$ pour $M \in \mathcal{K}_{\mathrm{MU}}$ est conséquence du cas $M=\mathrm{G}(S)$ par commutation de $\mathrm{T}_{W}$ aux coégalisateurs de 1-complexes (proposition 5.3.1).

Le point (b) du lemme est vérifié si $W^{\prime}$ est sans $p$ torsion et de cohomologie finie en chaque degré, car alors l'espace profini hom $\left(W^{\prime}, \mathrm{K}(S)\right)$ est équivalent homotopiquement à $\mathrm{K}^{a}\left(\widehat{\mathrm{L}}(S): \widehat{\mathrm{MU}}^{*} W^{\prime}\right)$.

Pour conclure rappelons que si $X$ est un espace profini pointé alors l'espace profini $\operatorname{hom}(W, X)$ est naturellement pointé par l'application constante. Soit $M$ une MUalgèbre instable augmentée. Le morphisme $\mathrm{T}_{W} M \rightarrow \mathrm{T}_{W} \widehat{\mathrm{MU}}^{*} \cong \widehat{\mathrm{MU}}^{*}$ fait de $\mathrm{T}_{W} M$ une MU-algèbre instable naturellement augmentée. Pour tout ensemble gradué $S$ l'isomorphisme $\mathrm{T}_{W} \mathrm{G}(S) \cong \widehat{\mathrm{MU}}^{*} \operatorname{hom}(W, \mathrm{~K}(S))$ respecte l'augmentation par construction. En utilisant le fait que le 1-cocomplexe $X \rightarrow \mathrm{K}\left(\widetilde{\widehat{\mathrm{MU}}^{*}} X\right) \stackrel{\mathrm{K}}{\leftrightarrows}\left(\widetilde{\mathrm{G}}\left(\widetilde{\mathrm{MU}^{*}} X\right)\right)$ de $\mathrm{h} \widehat{\mathcal{S}}_{\mathrm{pt}}$ induit un diagramme coégalisateur en MU-cohomologie continue et la commutation de $\mathrm{T}_{W}$ aux coégalisateurs de 1-complexes, on en déduit que le morphisme $\mathrm{T}_{W} \widehat{\mathrm{MU}}^{*} X \rightarrow \widehat{\mathrm{MU}}^{*} \operatorname{hom}(W, X)$ respecte l'augmentation. 


\section{CHAPITRE 2}

\section{COHOMOLOGIE DES ESPACES FONCTIONNELS DE SOURCE LE CLASSIFIANT D'UN GROUPE DE LIE COMPACT COMMUTATIF}

Nous appliquons le formalisme de la première partie et les résultats de $[\mathbf{D L}]$ à l'étude de la MU-cohomologie continue des espaces fonctionnels hom(B $\pi, X)$ pour $\pi$ un groupe de Lie compact commutatif.

La première section (6) rappelle (voir [DL]) l'analyse classique de la cohomologie modulo $p$ continue de l'espace fonctionnel $\operatorname{hom}\left(\mathrm{B} \mathbb{Z} / p^{n}, X\right)$ : elle est l'image par le foncteur exact Fix $: \mathrm{H}-\mathcal{U} \rightarrow \mathcal{U}$ de la cohomologie modulo $p$ continue équivariante de l'espace fonctionnel hom $\left(\mathrm{BZ} / p^{n-1}, X\right)$ pour une action du groupe $\mathbb{Z} / p$ à la source. On rappelle également les propriétés clé suivantes :

- Si $X$ est un espace profini fibrant sans $p$-torsion alors l'espace profini fonctionnel $\operatorname{hom}\left(\mathrm{BZ} / p^{n}, X\right)$ est également sans $p$-torsion.

- Si $X$ est un espace profini fibrant sans $p$-torsion alors la suite spectrale de Serre en cohomologie modulo $p$-continue associée à la fibration

$$
\operatorname{hom}\left(\mathrm{BZ} / p^{n}, X\right) \longrightarrow \operatorname{hom}\left(\mathrm{BZ} / p^{n}, X\right)_{\mathrm{h} \mathbb{Z} / p} \longrightarrow \mathrm{B} \mathbb{Z} / p
$$

dégénère au terme $\mathrm{E}_{2}$.

La section 7 étudie le foncteur $\mathrm{T}_{\mathrm{BZ} / p^{n}}: \mathcal{K}_{\mathrm{MU}} \rightarrow \mathcal{K}_{\mathrm{MU}}$ qu'on note $\mathrm{T}_{n}, n \in \mathbb{N} \cup\{\infty\}$. On montre d'abord que le morphisme

$$
\mathrm{T}_{n} \widehat{\mathrm{MU}}^{*} X \longrightarrow \widehat{\mathrm{MU}}^{*} \operatorname{hom}\left(\mathrm{BZ} / p^{n}, X\right)
$$

est un isomorphisme si $X$ est un espace profini fibrant sans p-torsion (théorème 7.1.1). Ce résultat se généralise au cas où la source est le classifiant d'un groupe de Lie compact commutatif $\pi$ en choisissant un morphisme $\mathbb{Z} / p^{n_{1}} \times \cdots \times \mathbb{Z} / p^{n_{r}} \rightarrow \pi$ induisant un isomorphisme entre les cohomologies modulo $p$ des classifiants. (Les exposants $n_{1}, \ldots, n_{r}$ peuvent prendre la valeur $\infty$.) On en déduit une version « $p$-complétée » de la proposition 7.17 de $[\mathbf{D L}]: \mathrm{Si} X$ est un espace sans $p$-torsion, l'application

$$
\operatorname{Hom}_{\mathrm{h} \mathcal{S}}\left(\mathrm{B} \pi, \widehat{X}^{p}\right) \longrightarrow \operatorname{Hom}_{\mathcal{K}_{\mathrm{MU}}}\left(\widehat{\mathrm{MU}}^{*} X, \widehat{\mathrm{MU}}^{*} \mathrm{~B} \pi\right)
$$

est une bijection, $\widehat{X}^{p}$ désignant le $p$-complété profini de $X$. 
Lorsque l'espace au but $X$ est quelconque, on utilise une MU-résolution libre de $X$ pour obtenir une suite spectrale aboutissant à la MU-cohomologie continue de l'espace fonctionnel $\operatorname{hom}(\mathrm{B} \pi, X)$ (section 7.2). La suite spectrale converge fortement si $X$ admet une MU-résolution libre de longueur finie. Nous montrons par exemple que si $\pi$ et $\pi^{\prime}$ sont deux groupes de Lie compacts commutatifs alors le morphisme

$$
\mathrm{T}_{\mathrm{B} \pi} \widehat{\mathrm{MU}}^{*} \mathrm{~B} \pi^{\prime} \longrightarrow \widehat{\mathrm{MU}}^{*} \operatorname{hom}\left(\mathrm{B} \pi, \mathrm{B} \pi^{\prime}\right)
$$

est encore un isomorphisme (proposition 7.2.5).

La section 7.3 mime algébriquement la description de l'espace profini fonctionnel $\operatorname{hom}\left(\mathrm{B} \mathbb{Z} / p^{n+1}, X\right)$ comme espace des points fixes homotopiques de l'espace profini $\operatorname{hom}\left(\mathrm{B} \mathbb{Z} / p^{n}, X\right)$ pour l'action de $\mathbb{Z} / p$ à la source : Il existe un foncteur $\mathcal{K}_{\mathrm{MU}} \rightarrow \mathrm{H}-\mathcal{U}$, que nous notons $M \mapsto \mathrm{H}_{\mathbb{Z} / p}\left(\mathrm{~T}_{n} M\right)(\mathrm{H}-\mathcal{U}$ désignant la catégorie des $\mathrm{H}$-modules instables sur l'algèbre de Steenrod modulo $p$ ), et un isomorphisme naturel

$$
\operatorname{Fix}_{\mathcal{U}} \mathrm{H}_{\mathbb{Z} / p}\left(\mathrm{~T}_{n} M\right) \stackrel{\sim}{\longrightarrow}\left(\mathrm{T}_{n+1} M\right) / \mathrm{f}^{1} .
$$

On obtient une propriété d'injectivité de la MU-cohomologie complétée en $p$ du classifiant d'un groupe de Lie compact commutatif (corollaire 7.3.6) proche du résultat de Wilkerson ([Wilk]) concernant la K-théorie du classifiant d'un tore comme $\lambda$-anneau.

La dernière section (8) démontre une formule d'adjonction pour le foncteur $\mathrm{T}_{n}$ en utilisant une propriété de platitude de la MU-cohomologie du classifiant de $\mathbb{Z} / p^{n}$ et la formule de Künneth qui s'en déduit, les deux dues à Landweber ([Lan]).

\section{Propriétés de la cohomologie modulo $p$ des espaces fonctionnels de source le classifiant de $\mathbb{Z} / p^{n}$ (d'après $[\mathbf{D L}]$ )}

Notons $\mathrm{H}$ la cohomologie modulo $p$ du groupe $\mathbb{Z} / p$ (autrement dit de l'espace classifiant $\mathrm{B} \mathbb{Z} / p)$. Le foncteur $\mathcal{K}_{\mathrm{H}} \rightarrow \mathcal{K}_{\mathrm{H}}, N \mapsto \mathrm{H} \otimes N$ admet un adjoint à gauche noté T. Nous rappelons ci-dessous les propriétés du foncteur T ([La1, chap. 2]) et son interprétation géométrique ([La1], $[\mathbf{D S}],[\mathbf{M o 2}])$.

Notons $\mathcal{U}$ la catégorie des modules instables sur l'algèbre de Steenrod modulo $p$ (voir par exemple $[\mathbf{S c}]$ ). La catégorie $\mathcal{U}$ est abélienne et on dispose d'un foncteur oubli $\mathcal{K}_{\mathrm{H}} \rightarrow \mathcal{U}$ au dessus de $\mathcal{E}$. Le foncteur (additif) $\mathcal{U} \rightarrow \mathcal{U}, N \mapsto H \otimes N$ admet également un adjoint à gauche $\mathrm{T}_{\mathcal{U}}$.

\section{THÉORÈME 6.1}

(a) Le foncteur $\mathrm{T}_{\mathcal{U}}$ est exact.

(b) Le diagramme

$$
\underset{\mathcal{U}}{\stackrel{\mathrm{K}_{\mathrm{U}}}{\longrightarrow} \underset{\mathcal{U}}{\stackrel{\mathrm{T}}{\longrightarrow}} \mathcal{K}_{\mathrm{H}}}
$$

commute. 
(c) Pour tout espace profini fibrant $X$ le morphisme

$$
\mathrm{TH}^{*} X \longrightarrow \mathrm{H}^{*} \operatorname{hom}(\mathrm{B} \mathbb{Z} / p, X)
$$

est un isomorphisme.

(Voir [La1, chap. 2] pour la démonstration des points (a) et (b) et [Mo2, $\S 2.2]$ pour la démonstration du point (c).)

Le foncteur T possède un analogue « équivariant» ([La1, chap. 4]) : Notons $\mathrm{H}-\mathcal{K}_{\mathrm{H}}$ (respectivement $\mathrm{H}-\mathcal{U}$ ) la catégorie des morphismes $\mathrm{H} \rightarrow M$ dans $\mathcal{K}_{\mathrm{H}}$ (autrement dit la catégorie des $\mathrm{H}$-algèbres instables) (respectivement la catégorie des $\mathrm{H}$-modules instables). Le foncteur $\mathcal{K}_{\mathrm{H}} \rightarrow \mathrm{H}-\mathcal{K}_{\mathrm{H}}$ (respectivement $\left.\mathcal{U} \rightarrow \mathrm{H}-\mathcal{U}\right), M \mapsto \mathrm{H} \otimes M$ admet un adjoint à gauche noté Fix (respectivement Fix $\mathcal{U}_{\text {). }}$

Soit $X$ un espace profini fibrant muni d'une action de $\mathbb{Z} / p$. On note $X_{\mathrm{h} \mathbb{Z} / p}$ la construction de Borel $\mathrm{E} \mathbb{Z} / p \times_{\mathbb{Z} / p} X$ et $X^{\mathrm{h} Z / p}$ l'espace profini des points fixes homotopiques $\operatorname{hom}_{\mathbb{Z} / p}(\mathrm{E} \mathbb{Z} / p, X)$ ( $c f$. la section 1.5); ce dernier s'interprète comme la fibre en l'identité de la fibration hom $\left(\mathrm{B} \mathbb{Z} / p, X_{\mathrm{h} Z} / p\right) \rightarrow \operatorname{hom}(\mathrm{B} \mathbb{Z} / p, \mathrm{~B} \mathbb{Z} / p)$. L'application d'évaluation $\mathrm{E} \mathbb{Z} / p \times X^{\mathrm{h} \mathbb{Z} / p} \rightarrow X$ induit un morphisme $\mathrm{B} \mathbb{Z} / p \times X^{\mathrm{h} \mathbb{Z} / p} \rightarrow X_{\mathrm{h} \mathbb{Z} / p}$ d'espaces profinis au dessus de $\mathrm{B} \mathbb{Z} / p$, lequel induit un morphisme $\operatorname{Fix}\left(\mathrm{H}^{*} X_{\mathrm{h} \mathbb{Z} / p}\right) \rightarrow \mathrm{H}^{*} X^{\mathrm{h} \mathbb{Z} / p}$.

\section{THÉORÈME 6.2}

(a) Le foncteur $\mathrm{Fix}_{\mathcal{U}}$ est exact.

(b) Le diagramme

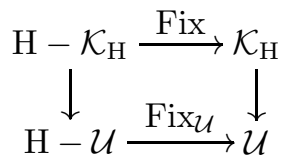

commute.

(c) Soit $X$ un espace profini fibrant muni d'une action de $\mathbb{Z} / p$; alors le morphisme $\operatorname{Fix}\left(\mathrm{H}^{*} X_{\mathrm{h} \mathbb{Z} / p}\right) \rightarrow \mathrm{H}^{*} X^{\mathrm{h} \mathbb{Z} / p}$ est un isomorphisme.

(Voir [La1, chap. 4] pour une démonstration des points (a), (b) et (c) lorsque $X$ est un espace $p$-fini. Le point (c) dans le cas général s'obtient en observant que si $X$ est un espace profini muni d'une action de $\mathbb{Z} / p$ alors sa résolution fibrante canonique $\mathrm{R} X$ est limite filtrante d'espaces $p$-finis avec action de $\mathbb{Z} / p$ et l'application $X \rightarrow \mathrm{R} X$ est une équivalence faible $\mathbb{Z} / p$-équivariante donc induit une équivalence faible entre les espaces profinis de points fixes homotopiques si $X$ est fibrant (voir la section 1.5).)

Passage du classifiant de $\mathbb{Z} / p$ au classifiant d'un p-groupe cyclique. - On dispose pour chaque entier $n \geqslant 0$ d'une suite exacte de pro- $p$-groupes abéliens simpliciaux $0 \rightarrow \mathrm{B} \mathbb{Z} / p^{n} \rightarrow \mathrm{B} \mathbb{Z} / p^{n+1} \rightarrow \mathrm{B} \mathbb{Z} / p \rightarrow 0$ de sorte que l'ensemble simplicial $\mathrm{B} \mathbb{Z} / p^{n}$ est, à une équivalence faible canonique près, muni d'une action de $\mathbb{Z} / p$ dont le quotient homotopique est BZZ $/ p^{n+1}$ ( $c f$. la section 1.5). Soit alors $X$ un espace profini fibrant. 
L'espace profini hom $\left(\mathrm{BZ} / p^{n+1}, X\right)$ s'interprète comme l'espace des points fixes homotopiques de l'espace profini hom $\left(\mathrm{BZ} / p^{n}, X\right)$ pour l'action de $\mathbb{Z} / p$ à la source $(c f$. [DL, chap. 3]). On dispose donc d'un isomorphisme

$$
\operatorname{Fix~} \mathrm{H}^{*}\left(\operatorname{hom}\left(\mathrm{BZ} / p^{n}, X\right)\right)_{\mathrm{h} \mathbb{Z} / p} \stackrel{\sim}{\longrightarrow} \mathrm{H}^{*} \operatorname{hom}\left(\mathrm{BZ} / p^{n+1}, X\right) .
$$

La $\mathbb{F}_{p^{-}}$-algèbre $\mathrm{H}^{*}\left(\operatorname{hom}\left(\mathrm{BZ} / p^{n}, X\right)\right)_{\mathrm{h} \mathbb{Z} / p}$ au dessous de $\mathrm{H}$ est l'aboutissement d'une suite spectrale de Serre de terme

$$
\mathrm{E}_{2}^{s, t} \cong \mathrm{H}^{s} \otimes \mathrm{H}^{t} \operatorname{hom}\left(\mathrm{BZ} / p^{n}, X\right)
$$

(il n'y a pas d'action du $\pi_{1}$ de la base parce que l'action de $\mathbb{Z} / p$ se prolonge en une action d'un groupe connexe).

Pour $n=0$ on a les isomorphismes hom $\left(\mathrm{BZ} / p^{0}, X\right) \cong X,\left(\operatorname{hom}\left(\mathrm{BZ} / p^{0}, X\right)\right)_{\mathrm{h} \mathbb{Z} / p} \cong$ $\mathrm{BZ} / p \times X, \operatorname{FixH}^{*}\left(\operatorname{hom}\left(\mathrm{BZ} / p^{0}, X\right)\right)_{\mathrm{h} \mathbb{Z} / p} \cong \mathrm{TH}^{*} X$.

Nous donnons deux exemples :

- Prenons $p=2$ et $X=\mathrm{B} \mathbb{Z} / 2$. L'espace hom(BZ/Z $/ 2, \mathrm{~B} \mathbb{Z} / 2)$ est la réunion disjointe de deux copies de $\mathrm{B} \mathbb{Z} / 2$. La première est munie de l'action triviale de $\mathbb{Z} / 2$ donc a un quotient homotopique égal au produit $\mathrm{B} \mathbb{Z} / 2 \times \mathrm{B} \mathbb{Z} / 2$ avec projection sur le premier facteur, donc la fibre en l'identité de la fibration

$$
\operatorname{hom}\left(\mathrm{BZ} / 2,(\mathrm{BZ} / 2)_{\mathrm{h} \mathbb{Z} / 2}\right) \longrightarrow \operatorname{hom}(\mathrm{BZ} / 2, \mathrm{~B} \mathbb{Z} / 2)
$$

s'identifie à l'espace hom $(\mathrm{B} \mathbb{Z} / 2, \mathrm{~B} \mathbb{Z} / 2) \cong \mathrm{B} \mathbb{Z} / 2 \sqcup \mathrm{B} \mathbb{Z} / 2$.

La seconde est munie de l'action non triviale de $\mathbb{Z} / 2$. Son quotient homotopique est l'espace $\mathrm{B} \mathbb{Z} / 4$ au dessus de $\mathrm{B} \mathbb{Z} / 2$. La fibre en l'identité de la fibration $\operatorname{hom}(\mathrm{B} \mathbb{Z} / 2, \mathrm{~B} \mathbb{Z} / 4) \rightarrow \operatorname{hom}(\mathrm{B} \mathbb{Z} / 2, \mathrm{~B} \mathbb{Z} / 2)$ est vide.

- Prenons $p=2$ et $X=\mathrm{BSU}(2)$. L'espace hom(BZ/2, $\mathrm{BSU}(2))$ est la réunion disjointe de deux copies de $\mathrm{BSU}(2)$. La première est munie de l'action triviale de $\mathbb{Z} / 2$ donc la fibre en l'identité correspondante s'identifie à l'espace

$$
\operatorname{hom}(\mathrm{BZ} / 2, \mathrm{BSU}(2)) \cong \mathrm{BSU}(2) \sqcup \mathrm{BSU}(2) .
$$

La seconde est munie d'une action non triviale de $\mathbb{Z} / 2$ dont le quotient homotopique est le classifiant du sous-groupe de $\mathrm{U}(2)$ formé des matrices de déterminant \pm 1 . La fibre en l'identité correspondante s'identifie au classifiant de $\mathrm{S}^{1}$.

Notons que les suites spectrales de Serre convergeant vers la cohomologie des constructions de Borel $\mathrm{BSU}(2)_{\mathrm{h} \mathbb{Z} / 2}$ sont en bidegrés pairs pour la première comme pour la seconde composante, donc dégénèrent au terme $\mathrm{E}_{2}$ donc sont identiques. On en déduit que les cohomologies des constructions de Borel sont isomorphes comme $\mathbb{F}_{2^{-}}$ algèbres graduées au dessous de $\mathrm{H}$, mais pas comme algèbres instables sur l'algèbre de Steenrod : La première est l'algèbre polynomiale en un générateur $u$ de degré 1 et en un générateur $v$ de degré 4 avec une action de l'algèbre de Steenrod déterminée par la relation $\mathrm{Sq}^{2} v=0$. La seconde est la même algèbre polynomiale avec une action de l'algèbre de Steenrod déterminée par $\mathrm{Sq}^{2} v=u^{2} v$. Elles sont différenciées par le foncteur Fix. 
Proposition 6.3 ([DL]). - Soient $n$ un entier positif et $X$ un espace profini fibrant sans p-torsion; alors :

(a) L'espace profini hom $\left(\mathrm{B} \mathbb{Z} / p^{n}, X\right)$ est sans $p$-torsion.

(b) La suite spectrale de Serre en cohomologie modulo $p$ continue de la fibration

$$
\operatorname{hom}\left(\mathrm{B} \mathbb{Z} / p^{n}, X\right) \longrightarrow \operatorname{hom}\left(\mathrm{B} \mathbb{Z} / p^{n}, X\right)_{\mathrm{h} \mathbb{Z} / p} \longrightarrow \mathrm{B} \mathbb{Z} / p
$$

dégénère au terme $\mathrm{E}_{2}$.

(Voir le théorème 7.14 et le scholie 7.15 de $[\mathbf{D L}]$.)

La dégénérescence de la suite spectrale de Serre entraîne des propriétés d'exactitude de la cohomologie modulo $p$ continue des espaces profinis hom $\left(\mathrm{BZ} / p^{n}, X\right)$ en la cohomologie modulo $p$ continue de $X$. Voici un premier résultat (comparer avec la proposition 4.6 de $[\mathbf{D L}])$ :

Corollaire 6.4. - Soient $n$ un entier positif et $X$ un espace profini fibrant sans p-torsion; alors l'objet simplicial augmenté de $\mathcal{E}$

$$
\mathrm{H}^{*} \operatorname{hom}\left(\mathrm{B} \mathbb{Z} / p^{n}, \mathrm{~K}^{\bullet}\left(\widehat{\mathrm{MU}}^{*} X\right)\right) \longrightarrow \mathrm{H}^{*} \operatorname{hom}\left(\mathrm{B} \mathbb{Z} / p^{n}, X\right)
$$

est acyclique.

(On rappelle que $X \rightarrow \mathrm{K}^{\bullet}\left(\widehat{\mathrm{MU}}^{*} X\right)$ désigne la MU-résolution cosimpliciale canonique de $X$, voir la section 4.2.)

Démonstration. - Le corollaire se démontre par récurrence sur $n$. Le diagramme $X \rightarrow \mathrm{K}^{\bullet}\left(\widehat{\mathrm{MU}}^{*} X\right)$ induit en MU-cohomologie continue une $\widehat{\mathrm{L}}$-algèbre simpliciale augmentée acyclique ( $c f$. la section 4.2$)$. Comme $X$ et les $\mathrm{K}^{k}\left(\widehat{\mathrm{MU}}^{*} X\right)$ sont sans $p$-torsion, l'objet simplicial augmenté $\mathrm{H}^{*} \mathrm{~K} \bullet\left(\widehat{\mathrm{MU}}^{*} X\right) \rightarrow \mathrm{H}^{*} X$ est acyclique dans $\mathcal{E}$ par le corollaire 4.2.2, ce qui donne le cas $n=0$.

Supposons le résultat vrai pour $n \geqslant 0$. On dispose d'un diagramme cosimplicial augmenté $\operatorname{hom}\left(\mathrm{B} \mathbb{Z} / p^{n}, X\right) \rightarrow \operatorname{hom}\left(\mathrm{B} \mathbb{Z} / p^{n}, \mathrm{~K} \bullet\left(\widehat{\mathrm{MU}}^{*} X\right)\right)$ d'espaces profinis sans $p$-torsion par le point (a) de la proposition 6.3 et avec action de $\mathbb{Z} / p$, induisant en cohomologie modulo $p$ continue un objet simplicial augmenté acyclique par hypothèse de récurrence. Le point (b) de la proposition 6.3 montre que pour chaque entier $k$ le $\mathbb{F}_{p}$-espace vectoriel $\mathrm{H}^{k} \operatorname{hom}\left(\mathrm{B} \mathbb{Z} / p^{n}, X\right)_{\mathrm{h} \mathbb{Z} / p}$ possède une filtration naturelle finie dont le gradué est l'objet $\oplus_{s+t=k} \mathrm{H}^{s} \otimes \mathrm{H}^{t}$ hom $\left(\mathrm{B} \mathbb{Z} / p^{n}, X\right)$. On en déduit par récurrence sur la filtration que le $\mathbb{F}_{p}$-espace vectoriel simplicial augmenté

$$
\mathrm{H}^{k} \operatorname{hom}\left(\mathrm{B} \mathbb{Z} / p^{n}, \mathrm{~K}^{\bullet}\left(\widehat{\mathrm{MU}}^{*} X\right)\right)_{\mathrm{h} \mathbb{Z} / p} \longrightarrow \mathrm{H}^{k} \operatorname{hom}\left(\mathrm{B} \mathbb{Z} / p^{n}, X\right)_{\mathrm{h} \mathbb{Z} / p}
$$

est acyclique, puis par le théorème 6.2 et l'interprétation de l'espace profini $\operatorname{hom}\left(\mathrm{B} \mathbb{Z} / p^{n+1}, X\right)$ comme espace de points fixes homotopiques, que l'objet simplicial augmenté

$$
\mathrm{H}^{*} \operatorname{hom}\left(\mathrm{BZ} / p^{n+1}, \mathrm{~K}^{\bullet}\left(\widehat{\mathrm{MU}}^{*} X\right)\right) \longrightarrow \mathrm{H}^{*} \operatorname{hom}\left(\mathrm{B} \mathbb{Z} / p^{n+1}, X\right)
$$

est acyclique dans $\mathcal{E}$. 
On obtient les mêmes informations sur la cohomologie modulo $p$ continue de l'espace fonctionnel hom $\left(\mathrm{BS}^{1}, X\right)$ en passant à la limite sur $n:$ Notons $\mathbb{Z} / p^{\infty}$ la colimite des groupes $\mathbb{Z} / p^{n}$ (donc le sous-groupe de $\mathcal{S}^{1}$ formé des éléments d'ordre une puissance de $p$ ). Le classifiant de $\mathbb{Z} / p^{\infty}$ est la colimite dans $\mathcal{S}$ des classifiants $\mathrm{B} \mathbb{Z} / p^{n}$; sa cohomologie modulo $p$ est la limite des cohomologie modulo $p$ des espaces $\mathrm{B} \mathbb{Z} / p^{n}$ (par dualité avec l'homologie modulo $p$ ). On en déduit que l'application $\mathrm{B} \mathbb{Z} / p^{\infty} \rightarrow \mathrm{BS}^{1}$ induit un isomorphisme en cohomologie modulo $p$, puis que pour tout espace profini fibrant $X$ l'application

$$
\operatorname{hom}\left(\mathrm{BS}^{1}, X\right) \longrightarrow \operatorname{hom}\left(\mathrm{B} \mathbb{Z} / p^{\infty}, X\right)
$$

est une équivalence faible dans $\widehat{\mathcal{S}}$ ( $c f$. le début de la section 1.3). Comme l'espace profini hom $\left(\mathrm{B} \mathbb{Z} / p^{\infty}, X\right)$ est la limite filtrante des espaces profinis hom $\left(\mathrm{B} \mathbb{Z} / p^{n}, X\right)$, le morphisme

$$
\operatorname{colim}_{n} \mathrm{H}^{*} \operatorname{hom}\left(\mathrm{B} \mathbb{Z} / p^{n}, X\right) \longrightarrow \mathrm{H}^{*} \operatorname{hom}\left(\mathrm{B} \mathbb{Z} / p^{\infty}, X\right)
$$

est un isomorphisme par le lemme 1.1.1 (voir aussi [DL, Lemme 3.7]).

\section{MU-cohomologie des espaces fonctionnels de source le classifiant d'un groupe de Lie compact commutatif}

7.1. Foncteurs $\mathrm{T}_{n}$ et cohomologie des espaces fonctionnels. - Soit $n$ un élément de $\mathbb{N} \cup\{\infty\}$. On pose $\mathrm{T}_{n}=\mathrm{T}_{\mathrm{BZ} / p^{n}}$ (cf. la section 5.3). On a donc pour tout espace profini fibrant $X$ un morphisme

$$
\mathrm{T}_{n} \widehat{\mathrm{MU}}^{*} X \longrightarrow \widehat{\mathrm{MU}}^{*} \operatorname{hom}\left(\mathrm{BZ} / p^{n}, X\right)
$$

qui est un isomorphisme si $X$ est l'image par K d'un ensemble gradué, et pour toute MU-algèbre instable $M$ une bijection naturelle

$$
\operatorname{Hom}_{\mathcal{K}_{\mathrm{MU}}}\left(\mathrm{T}_{n} M, \widehat{\mathrm{MU}}^{*}\right) \cong \operatorname{Hom}_{\mathcal{K}_{\mathrm{MU}}}\left(M, \widehat{\mathrm{MU}}^{*} \mathrm{~B} \mathbb{Z} / p^{n}\right) .
$$

Voici d'abord les propriétés formelles de $\mathrm{T}_{n}$ :

- Comme pour tout ensemble gradué $S$ l'espace profini hom $\left(\mathrm{B} \mathbb{Z} / p^{n}, \mathrm{~K}(S)\right)$ est sans $p$-torsion (proposition 6.3), le foncteur $\mathrm{T}_{n}$ commute aux colimites indexées par une catégorie petite par la proposition 5.3.1.

- Pour tout objet $M$ de $\mathcal{K}_{\mathrm{MU}}$ le morphisme $\operatorname{colim}_{n} \mathrm{~T}_{n} M \rightarrow \mathrm{T}_{\infty} M$ induit par les applications $\mathrm{B} \mathbb{Z} / p^{n} \rightarrow \mathrm{B} \mathbb{Z} / p^{\infty}$ est un isomorphisme dans $\widehat{\mathcal{M}}$ par le point (a) du lemme 5.3.3.

- Puisque l'espace $\mathrm{B} \mathbb{Z} / p^{\infty}$ est sans $p$-torsion et de cohomologie modulo $p$ finie en chaque degré, on a pour toute MU-algèbre instable $M$ un isomorphisme canonique $\mathrm{T}_{\infty} M \cong\left(M: \widehat{\mathrm{MU}}^{*} \mathrm{~B} \mathbb{Z} / p^{\infty}\right)_{\mathcal{K}_{\mathrm{MU}}}$

Rappelons qu'à toute MU-algèbre instable $M$ correspond fonctoriellement un diagramme cosimplicial $\mathrm{K} \bullet(M)$ de $\mathrm{h} \widehat{\mathcal{S}}$ vérifiant : 
- Pour tout entier $k$ l'espace profini $\mathrm{K}^{k}(M)$ est l'image par K d'un ensemble gradué. (En particulier $\mathrm{K}^{k}(M)$ est fibrant.)

- Le diagramme induit en MU-cohomologie continue est la G-algèbre simpliciale G. $(M)$.

THÉORÈme 7.1.1. - Soit $X$ un espace profini fibrant sans $p$-torsion et $n$ un élément de $\mathbb{N} \cup\{\infty\}$ alors le morphisme d'algèbres instables

$$
\mathrm{T}_{n} \widehat{\mathrm{MU}}^{*} X \longrightarrow \widehat{\mathrm{MU}}^{*} \operatorname{hom}\left(\mathrm{BZ} / p^{n}, X\right)
$$

est un isomorphisme.

Démonstration. - On dispose de la MU-résolution cosimpliciale $X \rightarrow \mathrm{K}^{\bullet}\left(\widehat{\mathrm{MU}}^{*} X\right)$ induisant en MU-cohomologie continue la G-résolution simpliciale de $\widehat{\mathrm{MU}}^{*} X$. Puisque $X$ est sans $p$-torsion, l'objet simplicial augmenté

$$
\mathrm{H}^{*} \operatorname{hom}\left(\mathrm{BZ} / p^{n}, \mathrm{~K}^{\bullet}\left(\widehat{\mathrm{MU}}^{*} X\right)\right) \longrightarrow \mathrm{H}^{*} \operatorname{hom}\left(\mathrm{BZ} / p^{n}, X\right)
$$

est acyclique par le corollaire 6.4. Puisque l'espace profini hom $\left(\mathrm{BZ} / p^{n}, X\right)$ est sans $p$-torsion, l'objet simplicial augmenté

$$
\widehat{\mathrm{MU}}^{*} \operatorname{hom}\left(\mathrm{B} \mathbb{Z} / p^{n}, \mathrm{~K} \cdot\left(\widehat{\mathrm{MU}}^{*} X\right)\right) \longrightarrow \widehat{\mathrm{MU}}^{*} \operatorname{hom}\left(\mathrm{B} \mathbb{Z} / p^{n}, X\right)
$$

est acyclique dans $\widehat{\mathcal{M}}$ par le corollaire 4.2.2. Puisque les objets simpliciaux $\mathrm{T}_{n} \mathrm{G} \cdot\left(\widehat{\mathrm{MU}}^{*} X\right)$ et $\widehat{\mathrm{MU}}^{*} \operatorname{hom}\left(\mathrm{BZ} / p^{n}, \mathrm{~K}^{\bullet}\left(\widehat{\mathrm{MU}}^{*} X\right)\right)$ sont canoniquement isomorphes, le morphisme entre les $\pi_{0}: \mathrm{T}_{n} \widehat{\mathrm{MU}}^{*} X \rightarrow \widehat{\mathrm{MU}}^{*} \operatorname{hom}\left(\mathrm{BZ} / p^{n}, X\right)$ est un isomorphisme.

On obtient par récurrence et en utilisant l'isomorphisme $\operatorname{hom}\left(W \times W^{\prime}, X\right) \cong$ $\operatorname{hom}\left(W, \operatorname{hom}\left(W^{\prime}, X\right)\right)$ pour $W, W^{\prime}$ dans $\mathcal{S}$ et $X$ dans $\widehat{\mathcal{S}}$ le :

Corollaire 7.1.2. - Soient $n_{1}, \ldots, n_{r}$ des éléments de $\mathbb{N} \cup\{\infty\}$ et $X$ un espace profini fibrant sans p-torsion, alors on a un isomorphisme canonique

$$
\mathrm{T}_{n_{1}} \cdots \mathrm{T}_{n_{k}} \widehat{\mathrm{MU}}^{*} X \stackrel{\sim}{\longrightarrow} \widehat{\mathrm{MU}}^{*} \operatorname{hom}\left(\mathrm{BZ} / p^{n_{1}} \times \cdots \times \mathrm{B} \mathbb{Z} / p^{n_{k}}, X\right) .
$$

Soit $\pi$ un groupe de Lie compact commutatif. Il existe une suite finie $n_{1}, \ldots, n_{r}$ d'éléments de $\mathbb{N} \cup\{\infty\}$ et un morphisme $\mathrm{B} \mathbb{Z} / p^{n_{1}} \times \cdots \times \mathrm{B} \mathbb{Z} / p^{n_{r}} \rightarrow \mathrm{B} \pi$ induisant un isomorphisme en cohomologie modulo $p$, donc induisant pour tout espace profini fibrant $X$ une équivalence faible

$$
\operatorname{hom}(\mathrm{B} \pi, X) \longrightarrow \operatorname{hom}\left(\mathrm{BZ} / p^{n_{1}} \times \cdots \times \mathrm{B} \mathbb{Z} / p^{n_{r}}, X\right)
$$

( $c f$. le début de la section 1.3) et pour toute MU-algèbre instable $M$ un isomorphisme naturel

$$
\mathrm{T}_{n_{1}} \cdots \mathrm{T}_{n_{r}} M \stackrel{\sim}{\longrightarrow} \mathrm{T}_{\mathrm{B} \pi} M
$$

par le point (b) du lemme 5.3.3 et le corollaire ci-dessus. On obtient : 
THÉORÈmE 7.1.3. - Soient $\pi$ un groupe de Lie compact commutatif et $X$ un espace profini fibrant sans p-torsion; alors le morphisme

$$
\mathrm{T}_{\mathrm{B} \pi} \widehat{\mathrm{MU}}^{*} X \longrightarrow \widehat{\mathrm{MU}}^{*} \operatorname{hom}(\mathrm{B} \pi, X)
$$

est un isomorphisme.

La proposition 5.3.2 entraîne immédiatement :

Corollaire 7.1.4. - Soient $\pi$ un groupe de Lie compact commutatif et $X$ un espace profini fibrant sans p-torsion; alors l'application

$$
\operatorname{Hom}_{\mathrm{h} \mathcal{S}}(\mathrm{B} \pi,|X|) \longrightarrow \operatorname{Hom}_{\mathcal{K}_{\mathrm{MU}}}\left(\widehat{\mathrm{MU}}^{*} X, \widehat{\mathrm{MU}}^{*} \mathrm{~B} \pi\right)
$$

est une bijection.

7.2. Résolution de l'espace au but. - La proposition suivante est une généralisation du corollaire 7.9 de $[\mathbf{D L}]$ :

Proposition 7.2.1. - Soient $\pi$ un groupe de Lie compact commutatif et $X \rightarrow Y$ un morphisme entre espaces profinis dont on note $C$ la cofibre homotopique; alors le morphisme de la cofibre homotopique de l'application $\operatorname{hom}(\mathrm{B} \pi, \mathrm{R} X) \rightarrow \operatorname{hom}(\mathrm{B} \pi, \mathrm{R} Y)$ dans l'espace profini hom $(\mathrm{B} \pi, \mathrm{RC})$ est une équivalence faible de $\widehat{\mathcal{S}}_{\mathrm{pt}}$.

Démonstration. - On démontre d'abord le résultat pour $\pi=\mathbb{Z} / p^{n}$ par récurrence sur $n$. Pour $n=0$ il n'y a rien à démontrer.

Supposons le résultat vrai pour $n \geqslant 0$. Le diagramme d'espaces profinis avec action de $\mathbb{Z} / p$

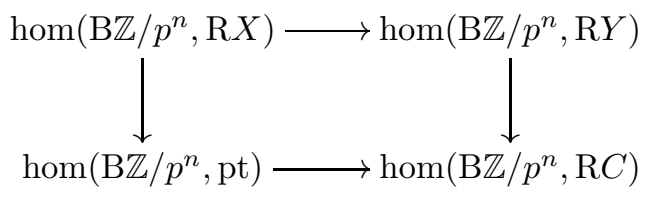

est alors homotopiquement cocartésien. Par commutation des colimites entre elles, il induit un diagramme homotopiquement cocartésien

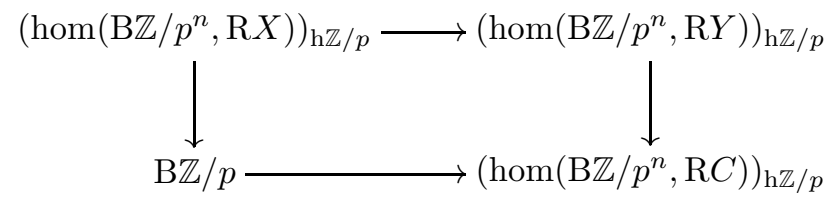

d'espaces profinis au dessus de $\mathrm{B} \mathbb{Z} / p$. On dispose donc d'une suite exacte longue de Mayer-Vietoris dans la catégorie $\mathrm{H}-\mathcal{U}$. L'exactitude du foncteur Fix $\mathcal{U}$, sa commutation à la suspension ([La1, cor. 4.6.2.2]) et son interprétation géométrique donnent alors le résultat pour $n+1$.

Le cas $\pi=\mathbb{Z} / p^{\infty}$ s'obtient par passage à la limite ( $c f$. la fin de la section 6 ). Le cas $\pi$ quelconque s'obtient en choisissant un morphisme $\mathrm{B} \mathbb{Z} / p^{n_{1}} \times \cdots \times \mathrm{B} \mathbb{Z} / p^{n_{r}} \rightarrow \mathrm{B} \pi$ 
induisant un isomorphisme en cohomologie modulo $p$, en utilisant l'isomorphisme $\operatorname{hom}\left(W \times W^{\prime}, X^{\prime}\right) \cong \operatorname{hom}\left(W, \operatorname{hom}\left(W^{\prime}, X^{\prime}\right)\right)$ et une récurrence sur $r$.

LEMme 7.2.2. - Soient $\pi$ un groupe de Lie compact commutatif et $X \rightarrow X^{0}$ un morphisme entre espaces profinis tel que $X^{0}$ est sans p-torsion et tel que le morphisme $\widehat{\mathrm{MU}}^{*} X^{0} \rightarrow \widehat{\mathrm{MU}}^{*} X$ est un épimorphisme dans $\widehat{\mathcal{M}}$; alors les deux conditions suivantes sont équivalentes :

(i) Le morphisme $\mathrm{T}_{\mathrm{B} \pi} \widehat{\mathrm{MU}}^{*} \mathrm{R} X \rightarrow \widehat{\mathrm{MU}}^{*} \operatorname{hom}(\mathrm{B} \pi, \mathrm{R} X)$ est un épimorphisme dans $\widehat{\mathcal{M}}$.

(ii) Le morphisme $\widehat{\mathrm{MU}}^{*} \operatorname{hom}\left(\mathrm{B} \pi, \mathrm{R} X^{0}\right) \rightarrow \widehat{\mathrm{MU}}^{*} \operatorname{hom}(\mathrm{B} \pi, \mathrm{R} X)$ est un épimorphisme dans $\widehat{\mathcal{M}}$.

Démonstration. - Le morphisme $\mathrm{T}_{\mathrm{B} \pi} \widehat{\mathrm{MU}}^{*} X^{0} \rightarrow \widehat{\mathrm{MU}}^{*} \mathrm{hom}\left(\mathrm{B} \pi, X^{0}\right)$ est un isomorphisme (théorème 7.1.3) donc la condition (ii) implique (i).

Inversement tout morphisme de $\mathcal{K}_{\mathrm{MU}}$ qui est épi dans $\widehat{\mathcal{M}}$ est le coégalisateur d'un 1-complexe de $\mathcal{K}_{\mathrm{MU}}$. Comme $\mathrm{T}_{\mathrm{B} \pi}$ commute aux coégalisateurs de 1-complexes (proposition 5.3.1 (a)), l'image par $\mathrm{T}_{\mathrm{B} \pi}$ de ce morphisme est encore un épimorphisme dans $\widehat{\mathcal{M}}$. Comme le morphisme $\widehat{\mathrm{MU}}^{*} X^{0} \rightarrow \widehat{\mathrm{MU}}^{*} X$ est épi par hypothèse, la condition (i) implique la condition (ii).

Proposition 7.2.3. - Soient $\pi$ un groupe de Lie compact commutatif et $X \rightarrow X^{0}$ une cofibration dans $\widehat{\mathcal{S}}$ tel que $X^{0}$ est sans $p$-torsion et tel que le morphisme $\widehat{\mathrm{MU}}^{*} X^{0} \rightarrow$ $\widehat{\mathrm{MU}}^{*} X$ est un épimorphisme dans $\widehat{\mathcal{M}}$. On suppose que le morphisme $\mathrm{T}_{\mathrm{B} \pi} \widehat{\mathrm{MU}}^{*} Y \rightarrow$ $\widehat{\mathrm{MU}}^{*} \operatorname{hom}(\mathrm{B} \pi, Y)$ est un épimorphisme dans $\widehat{\mathcal{M}}$ pour $Y=\mathrm{R} X$ et $Y=\mathrm{R}\left(X^{0} / X\right)$; alors le morphisme $\mathrm{T}_{\mathrm{B} \pi} \widehat{\mathrm{MU}}^{*} X \rightarrow \widehat{\mathrm{MU}}^{*} \operatorname{hom}(\mathrm{B} \pi, \mathrm{R} X)$ est un isomorphisme

Démonstration. - Puisque la suite $\widetilde{\mathrm{MU}^{*}} X^{0} / X \rightarrow \widehat{\mathrm{MU}}^{*} X^{0} \rightarrow \widehat{\mathrm{MU}}^{*} X \rightarrow 0$ est exacte, le diagramme $X \rightarrow X^{0} \leftrightarrows X^{0} \times\left(X^{0} / X\right)$ induit un diagramme coégalisateur

$$
\widehat{\mathrm{MU}}^{*} X^{0} \widehat{\otimes} \widehat{\mathrm{MU}}^{*}\left(X^{0} / X\right) \rightleftarrows \widehat{\mathrm{MU}}^{*} X^{0} \longrightarrow \widehat{\mathrm{MU}}^{*} X
$$

dans $\widehat{\mathcal{M}}$ comme dans $\mathcal{K}_{\mathrm{MU}}$. (On n'a pas besoin d'une formule de Künneth pour cela.) Comme le foncteur $\mathrm{T}_{\mathrm{B} \pi}: \mathcal{K}_{\mathrm{MU}} \rightarrow \mathcal{K}_{\mathrm{MU}}$ et l'oubli $\mathcal{K}_{\mathrm{MU}} \rightarrow \widehat{\mathcal{M}}$ commutent aux coégalisateurs de 1-complexes, le diagramme image par $\mathrm{T}_{\mathrm{B} \pi}$ est encore coégalisateur dans $\widehat{\mathcal{M}}$.

Le morphisme $\widehat{\mathrm{MU}}^{*} \operatorname{hom}\left(\mathrm{B} \pi, \mathrm{R} X^{0}\right) \rightarrow \widehat{\mathrm{MU}}^{*} \operatorname{hom}(\mathrm{B} \pi, \mathrm{R} X)$ est un épimorphisme dans $\widehat{\mathcal{M}}$ par le lemme 7.2.2. La proposition 7.2.1 montre alors que la $\widehat{\mathrm{L}}$-algèbre $\widehat{\mathrm{MU}}^{*} \operatorname{hom}(\mathrm{B} \pi, \mathrm{R} X)$ est le conoyau du morphisme

$$
{\widetilde{\mathrm{MU}^{*}}}^{h o m}\left(\mathrm{~B} \pi, \mathrm{R}\left(X^{0} / X\right)\right) \longrightarrow \widetilde{\widehat{\mathrm{MU}}}^{*} \operatorname{hom}\left(\mathrm{B} \pi, \mathrm{R} X^{0}\right) \text {, }
$$


donc que le diagramme

$$
\begin{aligned}
\widehat{\mathrm{MU}}^{*} \operatorname{hom}\left(\mathrm{B} \pi, \mathrm{R} X^{0}\right) \widehat{\otimes} \widehat{\mathrm{MU}}^{*} \operatorname{hom}\left(\mathrm{B} \pi, \mathrm{R}\left(X^{0} / X\right)\right) \rightleftarrows \widehat{\mathrm{MU}}^{*} & \operatorname{hom}\left(\mathrm{B} \pi, \mathrm{R} X^{0}\right) \\
& \longrightarrow \widehat{\mathrm{MU}}^{*} \operatorname{hom}(\mathrm{B} \pi, \mathrm{R} X)
\end{aligned}
$$

est un diagramme coégalisateur dans $\widehat{\mathcal{M}}$. La proposition résulte maintenant du lemme suivant :

LEMME 7.2.4. - Soit $\left(M_{1} \rightleftarrows M_{0}\right) \rightarrow\left(N_{1} \rightleftarrows N_{0}\right)$ un morphisme entre 1-complexes d'une catégorie abélienne dont on note $M$ et $N$ les coégalisateurs respectifs. On suppose que les morphismes $M_{1} \rightarrow N_{1}$ et $M \rightarrow N$ sont épi et que le morphisme $M_{0} \rightarrow N_{0}$ est iso; alors le morphisme $M \rightarrow N$ est un isomorphisme.

Application. - Soient $\pi$ un groupe de Lie compact commutatif et $X$ un espace profini pointé dont la MU-cohomologie est nulle en degré impair. Soit $S$ l'ensemble gradué égal à $\widetilde{\widehat{M U}}^{*} X$ en degré pair et égal à l'ensemble vide en degré impair. La cohomologie modulo $p$ continue de l'espace profini $\mathrm{K}(S)$ est nulle en degré impair (le cas où $S$ est réduit à un élément résulte de $[\mathbf{W i}]$ ), donc également sa MU-cohomologie continue. Le morphisme $S \rightarrow \widehat{\widehat{\mathrm{MU}}}^{*} X$ correspond à un morphisme $X \rightarrow \mathrm{K}(S)$ dans $\mathrm{h} \widehat{\mathcal{S}}_{\mathrm{pt}}$, lequel induit un épimorphisme $\widetilde{\mathrm{G}}(S) \rightarrow \widehat{\widehat{\mathrm{MU}}}^{*} X$ dans $\widehat{\mathcal{M}}$. On en déduit que la MU-cohomologie continue de la cofibre de l'application $X \rightarrow \mathrm{K}(S)$ est encore nulle en degré impair, puis que $X$ admet une MU-résolution libre par des espaces profinis ayant cette propriété.

Choisissons une telle résolution $\left(C^{k-1} \rightarrow X^{k}\right)_{k}$ (avec les notations de la section 4.2). Comme la cohomologie modulo $p$ continue des espaces profinis $X^{k}$ est nulle en degré impair, il en est de même de celle des espaces profinis $\operatorname{hom}\left(\mathrm{B} \pi, \mathrm{R} X^{k}\right)$ par le théorème 3.10 de $[\mathbf{D L}]$ donc de leur MU-cohomologie continue.

Supposons que la MU-cohomologie continue des espaces profinis $\operatorname{hom}\left(\mathrm{B} \pi, \mathrm{R} C^{k}\right)$ est également nulle en degré impair. La proposition 7.2.1 montre alors que la suite $C^{k-1} \rightarrow X^{k} \rightarrow C^{k}$ induit une suite exacte courte de $\widehat{\mathrm{L}}$-algèbres

$0 \longrightarrow \widetilde{\widehat{\mathrm{MU}}}^{*} \operatorname{hom}\left(\mathrm{B} \pi, \mathrm{R} C^{k}\right) \longrightarrow \widehat{\mathrm{MU}}^{*} \operatorname{hom}\left(\mathrm{B} \pi, \mathrm{R} X^{k}\right) \longrightarrow \widehat{\mathrm{MU}}^{*} \operatorname{hom}\left(\mathrm{B} \pi, \mathrm{R} C^{k-1}\right) \longrightarrow 0$.

Le morphisme $\mathrm{T}_{\mathrm{B} \pi} \widehat{\mathrm{MU}}^{*} C^{k-1} \rightarrow \widehat{\mathrm{MU}}^{*} \operatorname{hom}\left(\mathrm{B} \pi, \mathrm{R} C^{k-1}\right)$ est donc un épimorphisme dans $\widehat{\mathcal{M}}$ par le lemme 7.2.2. La proposition 7.2.3 permet de conclure que le morphisme $\mathrm{T}_{\mathrm{B} \pi} \widehat{\mathrm{MU}}^{*} X \rightarrow \widehat{\mathrm{MU}}^{*} \operatorname{hom}(\mathrm{B} \pi, X)$ est un isomorphisme.

La proposition suivante et son corollaire illustrent notre discussion :

Proposition 7.2.5. — Soient $\pi, \pi^{\prime}$ deux p-groupes abéliens finis; alors le morphisme

$$
\mathrm{T}_{\mathrm{B} \pi} \widehat{\mathrm{MU}}^{*} \mathrm{~B} \pi^{\prime} \longrightarrow \widehat{\mathrm{MU}}^{*} \operatorname{hom}\left(\mathrm{B} \pi, \mathrm{B} \pi^{\prime}\right)
$$

est un isomorphisme. 
Démonstration. - Commençons par le cas où $\pi^{\prime}$ est un $p$-groupe cyclique d'ordre $n$. Notons $\eta$ le fibré en droite canonique au dessus de $\mathrm{BS}^{1}$. La cofibre de l'application $\mathrm{BZ} / p^{n} \rightarrow \mathrm{BS}^{1}$ s'interprète comme l'espace de $\mathrm{Thom}^{\mathrm{Th}} \mathrm{h}_{n}$ du fibré $\eta^{\otimes p^{n}}$ (le produit tensoriel de $p^{n}$-copies de $\eta$ ), donc est sans $p$-torsion et de MU-cohomologie (complétée en $p$ ) concentrée en degré pair. L'espace $\operatorname{hom}\left(\mathrm{B} \pi, \mathrm{B} \mathbb{Z} / p^{n}\right)$ est isomorphe au produit de l'ensemble simplicial constant $\operatorname{Hom}_{\mathrm{ab}}\left(\pi, \mathbb{Z} / p^{n}\right)$ par $\mathrm{B} \mathbb{Z} / p^{n}$; sa MU-cohomologie complétée en $p$ est donc concentrée en degré pair. Puisque $\mathrm{Th}_{n}$ est sans $p$-torsion et de cohomologie modulo $p$ concentrée en degré pair, la MU-cohomologie de l'espace profini hom $\left(\mathrm{B} \pi, \mathrm{RTh}_{n}\right)$ est également concentrée en degré pair, d'où le résultat par la discussion qui précède.

Le cas $\pi^{\prime} p$-groupe abélien fini quelconque s'obtient en choisissant un isomorphisme entre $\pi^{\prime}$ et un produit de $p$-groupes cycliques, en observant que les foncteurs $\pi^{\prime} \mapsto$ $\widehat{\mathrm{MU}}^{*} \mathrm{~B} \pi^{\prime}$ et $\pi^{\prime} \mapsto \widehat{\mathrm{MU}}^{*} \operatorname{hom}\left(\mathrm{B} \pi, \mathrm{B} \pi^{\prime}\right)$ transforment un produit de $p$-groupes cycliques en la somme des images dans $\mathcal{K}_{\mathrm{MU}}$ par le point (b) de la proposition 8.3 plus loin, et en observant que le foncteur $\mathrm{T}_{\mathrm{B} \pi}$ commute aux sommes dans $\mathcal{K}_{\mathrm{MU}}$ (voir le début de la section 7).

La proposition 5.3.2 entraîne :

Corollaire 7.2.6. — Soient $\pi, \pi^{\prime}$ deux p-groupes abéliens finis; alors l'application

$$
\operatorname{Hom}_{\mathrm{h} \mathcal{S}}\left(\mathrm{B} \pi, \mathrm{B} \pi^{\prime}\right) \longrightarrow \operatorname{Hom}_{\mathcal{K}_{\mathrm{MU}}}\left(\widehat{\mathrm{MU}}^{*} \mathrm{~B} \pi^{\prime}, \widehat{\mathrm{MU}}^{*} \mathrm{~B} \pi\right)
$$

est une bijection.

Question. - Si $X$ est un espace profini fibrant dont la MU-cohomologie continue est nulle en degré impair, en est il de même de l'espace profini hom $(\mathrm{B} \pi, X)$ ?

Les travaux de Ravenel-Wilson-Yagita $([\mathbf{R W Y}])$ montrent que la réponse est oui si $X$ est un espace d'Eilenberg-MacLane $\mathrm{K}\left(\mathbb{Z} / p^{k}, l\right), k, l \geqslant 0$.

Plus généralement la proposition 7.2.1 montre que le foncteur

$$
X \longmapsto \widehat{\widehat{\mathrm{MU}}}^{*} \operatorname{hom}(\mathrm{B} \pi, \mathrm{R} X)
$$

est une théorie cohomologique réduite sur h $\widehat{\mathcal{S}}_{\mathrm{pt}}$. A toute MU-résolution libre $X \rightarrow X^{*}$ d'un espace profini pointé $X$ correspond une suite spectrale aboutissant à un gradué de la $\widehat{\mathrm{L}}$-algèbre $\widetilde{\widehat{\mathrm{MU}}}^{*} \operatorname{hom}(\mathrm{B} \pi, \mathrm{R} X)$ (voir la section 4.3 ).

Notons, pour $M$ une MU-algèbre instable augmentée, $\widetilde{\mathrm{T}}_{\mathrm{B} \pi} M$ le noyau de l'augmentation $\mathrm{T}_{\mathrm{B} \pi} M \rightarrow \widehat{\mathrm{MU}}^{*}$ (voir la fin de la section 5.3). Le terme $\mathrm{E}_{2}^{-s, *}$ de la suite spectrale s'identifie au $s$-ième objet d'homologie du complexe de $\widehat{\mathrm{L}}$-algèbres $\widetilde{\mathrm{T}}_{\mathrm{B} \pi}\left(\widehat{\mathrm{MU}}^{*} X^{*}\right)$.

Cette suite spectrale converge fortement vers la MU-cohomologie continue réduite de l'espace profini pointé $\operatorname{hom}(\mathrm{B} \pi, \mathrm{R} X)$ si la MU-résolution libre $X \rightarrow X^{*}$ est de longueur finie. 
7.3. Liens entre les foncteurs $T_{n}$

Proposition 7.3.1. - On a pour toute MU-algèbre instable $M$ un isomorphisme naturel

$$
\mathrm{T}_{\mathcal{U}}\left(M / \mathrm{f}^{1}\right) \cong\left(\mathrm{T}_{1} M\right) / \mathrm{f}^{1}
$$

dans $\mathcal{U}$.

Démonstration. - On a pour tout ensemble gradué $S$ des isomorphismes canoniques

- $\mathrm{G}(S) / \mathrm{f}^{1} \stackrel{\sim}{\longrightarrow} \mathrm{H}^{*} \mathrm{~K}(S)$ car $\mathrm{K}(S)$ est sans $p$-torsion,

- $\mathrm{T}_{\mathcal{U}} \mathrm{H}^{*} \mathrm{~K}(S) \stackrel{\sim}{\longrightarrow} \mathrm{H}^{*} \operatorname{hom}(\mathrm{B} \mathbb{Z} / p, \mathrm{~K}(S))$ par le théorème 6.1,

- $\left(\widehat{\mathrm{MU}}^{*} \operatorname{hom}(\mathrm{BZ} / p, \mathrm{~K}(S))\right) / \mathrm{f}^{1} \stackrel{\sim}{\longrightarrow} \mathrm{H}^{*} \operatorname{hom}(\mathrm{B} \mathbb{Z} / p, \mathrm{~K}(S))$ car l'espace profini fonctionnel hom $(\mathrm{BZZ} / p, \mathrm{~K}(S))$ est sans $p$-torsion (proposition 6.3) et

- $\mathrm{T}_{1} \mathrm{G}(S) \stackrel{\sim}{\longrightarrow} \widehat{\mathrm{MU}}^{*} \operatorname{hom}(\mathrm{B} \mathbb{Z} / p, \mathrm{~K}(S))$ par construction de $\mathrm{T}_{1}$.

On en déduit un isomorphisme canonique $\mathrm{T}_{\mathcal{U}}\left(\mathrm{G}(S) / \mathrm{f}^{1}\right) \cong\left(\mathrm{T}_{1} \mathrm{G}(S)\right) / \mathrm{f}^{1}$. Le cas $M$ quelconque s'obtient en utilisant le début de la G-résolution libre de $M$ et la commutation des foncteurs considérés aux coégalisateurs de 1-complexes.

Soit $n \geqslant 1$ un entier. A tout espace profini fibrant $X$ correspondent les objets $\mathrm{H}^{*} \operatorname{hom}\left(\mathrm{B} \mathbb{Z} / p^{n}, X\right)$ de $\mathcal{U}$ et $\mathrm{H}^{*}\left(\operatorname{hom}\left(\mathrm{B} \mathbb{Z} / p^{n}, X\right)\right)_{\mathrm{h} \mathbb{Z} / p}$ de $\mathrm{H}-\mathcal{U}$ avec, si $X$ est sans $p$-torsion, les propriétés suivantes :

(1) On a un isomorphisme canonique

$$
\operatorname{Fix}_{\mathcal{U}} \mathrm{H}^{*}\left(\operatorname{hom}\left(\mathrm{B} \mathbb{Z} / p^{n}, X\right)\right)_{\mathrm{h} \mathbb{Z} / p} \cong \mathrm{H}^{*} \operatorname{hom}\left(\mathrm{B} \mathbb{Z} / p^{n+1}, X\right) .
$$

(2) Pour tout entier $k$ le $\mathbb{F}_{p}$-espace vectoriel $\mathrm{H}^{k}\left(\operatorname{hom}\left(\mathrm{BZ} / p^{n}, X\right)\right)_{\mathrm{h} \mathbb{Z} / p}$ a une filtration finie naturelle dont le gradué s'identifie à $\oplus_{s+t=k} \mathrm{H}^{s} \otimes \mathrm{H}^{t} \operatorname{hom}\left(\mathrm{BZ} / p^{n}, X\right)$.

(3) On a un isomorphisme canonique

$$
\left(\mathrm{MU}^{*} \operatorname{hom}\left(\mathrm{B} \mathbb{Z} / p^{n}, X\right)\right) / \mathrm{f}^{1} \cong \mathrm{H}^{*} \operatorname{hom}\left(\mathrm{BZ} / p^{n}, X\right)
$$

dans $\mathcal{U}$

(La propriété (1) vient de l'interprétation de l'espace profini hom $\left(\mathrm{BZ} / p^{n+1}, X\right)$ comme espace de points fixes homotopiques; la propriété (2) vient de la dégénérescence de la suite spectrale de Serre (proposition 6.3); la propriété (3) vient de ce que l'espace profini $\operatorname{hom}\left(\mathrm{BZ} / p^{n}, X\right)$ est sans $p$-torsion. Voir la section 6 .)

Soit $M$ une MU-algèbre instable. On définit l'objet $\mathrm{H}_{\mathbb{Z} / p}\left(\mathrm{~T}_{n} M\right)$ de $\mathrm{H}-\mathcal{U}$ comme le coégalisateur du 1-complexe

$$
\mathrm{H}^{*}\left(\operatorname{hom}\left(\mathrm{BZ} / p^{n}, \mathrm{~K}(\mathrm{G}(M))\right)\right)_{\mathrm{h} \mathbb{Z} / p} \rightleftarrows \mathrm{H}^{*}\left(\operatorname{hom}\left(\mathrm{B} \mathbb{Z} / p^{n}, \mathrm{~K}(M)\right)\right)_{\mathrm{h} \mathbb{Z} / p}
$$

induit par le 1-cocomplexe $\mathrm{K}(M) \leftrightarrows \mathrm{K}(\mathrm{G}(M))$ de $\mathrm{h} \widehat{\mathcal{S}}$.

Pour chaque entier $k$ le $\mathbb{F}_{p}$-espace vectoriel formé des éléments en degré $k$ de $\mathrm{H}_{\mathbb{Z} / p}\left(\mathrm{~T}_{n} M\right)$ hérite de $\mathrm{H}^{k}\left(\operatorname{hom}\left(\mathrm{BZ} / p^{n}, \mathrm{~K}(M)\right)\right)_{\mathrm{h} \mathbb{Z} / p}$ d'une filtration finie naturelle.

Pour tout espace profini fibrant $X$ l'unité $X \rightarrow \mathrm{K}\left(\widehat{\mathrm{MU}}^{*} X\right)$ induit un morphisme $\mathrm{H}_{\mathbb{Z} / p}\left(\mathrm{~T}_{n} \widehat{\mathrm{MU}}^{*} X\right) \rightarrow \mathrm{H}^{*}\left(\operatorname{hom}\left(\mathrm{BZ} / p^{n}, X\right)\right)_{\mathrm{h} \mathbb{Z} / p}$. Par construction ce morphisme est un 
isomorphisme si $X$ est l'image par K d'un ensemble gradué et ce de façon compatible avec la filtration en chaque degré. Également par construction l'image par $\mathrm{H}_{\mathbb{Z} / p}\left(\mathrm{~T}_{n}-\right)$ du diagramme $\mathrm{G}^{2}(M) \leftrightarrows \mathrm{G}(M) \rightarrow M$ associé à l'algèbre instable $M$ est un diagramme coégalisateur dans $\mathrm{H}-\mathcal{U}$ ( $c f$. la proposition A.2.2 et sa démonstration).

Les propriétés (1) et (3) ci-dessus donnent pour tout ensemble gradué $S$ un isomorphisme $\operatorname{Fix}_{\mathcal{U}} \mathrm{H}_{\mathbb{Z} / p}\left(\mathrm{~T}_{n} \mathrm{G}(S)\right) \cong\left(\mathrm{T}_{n+1} \mathrm{G}(S)\right) / \mathrm{f}^{1}$ naturel en $\mathrm{G}(S) \in \mathcal{K}_{\mathrm{MU}}$. La propriété (2) montre que le gradué de la filtration du $\mathbb{F}_{p}$-espace vectoriel formé des éléments de degré $k$ de $\mathrm{H}_{\mathbb{Z} / p}\left(\mathrm{~T}_{n} \mathrm{G}(S)\right)$ est naturellement isomorphe à $\oplus_{s+t=k} \mathrm{H}^{s} \otimes\left(\left(\mathrm{T}_{n} \mathrm{G}(S)\right) / \mathrm{f}^{1}\right)^{t}$. En passant aux coégalisateurs on obtient la :

Proposition 7.3.2. - Soit $M$ une MU-algèbre instable. On a un isomorphisme naturel

$$
\operatorname{Fix}_{\mathcal{U}} \mathrm{H}_{\mathbb{Z} / p}\left(\mathrm{~T}_{n} M\right) \cong\left(\mathrm{T}_{n+1} M\right) / \mathrm{f}^{1} .
$$

Enfin puisque le foncteur $\widehat{\mathcal{M}} \rightarrow \mathcal{E}, M \mapsto M / \mathrm{f}^{1}$ commute aux colimites filtrantes (lemme 2.2.2), le lemme 5.3.3 entraîne la

Proposition 7.3.3. - Soit M une MU-algèbre instable. Le morphisme

$$
\operatorname{colim}_{n}\left(\left(\mathrm{~T}_{n} M\right) / \mathrm{f}^{1}\right) \longrightarrow\left(\mathrm{T}_{\infty} M\right) / \mathrm{f}^{1}
$$

est un isomorphisme dans $\mathcal{U}$.

Proposition 7.3.4. - Soient $n$ un entier et $M$ une MU-algèbre instable dont la $\widehat{\mathrm{L}}$-algèbre sous-jacente est dans $\widehat{\mathcal{L}}$; alors

(a) L'objet simplicial augmenté $\left(\mathrm{T}_{n} \mathrm{G} \cdot(M)\right) / \mathrm{f}^{1} \rightarrow\left(\mathrm{T}_{n} M\right) / \mathrm{f}^{1}$ de $\mathcal{E}$ est acyclique.

(b) La $\widehat{\mathrm{L}}$-algèbre $\mathrm{T}_{n} M$ est dans $\widehat{\mathcal{L}}$.

(c) Pour tout entier $k$ le gradué de la filtration du $\mathbb{F}_{p}$-espace vectoriel $\left(\mathrm{H}_{\mathbb{Z} / p}\left(\mathrm{~T}_{n} M\right)\right)^{k}$ est naturellement isomorphe $\grave{a} \oplus_{s+t=k} \mathrm{H}^{s} \otimes\left(\left(\mathrm{T}_{n} M\right) / \mathrm{f}^{1}\right)^{t}$.

Démonstration. - Le point (b) est conséquence du point (a), du fait que $\mathrm{T}_{n} \mathrm{G}_{\bullet}(M)$ est un objet simplicial de $\widehat{\mathcal{L}}$ et du point (a) de la proposition 4.2.1.

On démontre les points (a) et (c) par récurrence sur $n$. Puisque G. $(M) \rightarrow M$ est acyclique dans $\widehat{\mathcal{M}},\left(\mathrm{G}_{\bullet}(M)\right) / \mathrm{f}^{1} \rightarrow M / \mathrm{f}^{1}$ est acyclique dans $\mathcal{E}$ par le point (a) de la proposition 4.2.1. On en déduit le point (a) pour $n=1$ par la proposition 7.3 .1 et l'exactitude du foncteur $\mathrm{T}_{\mathcal{U}}$.

Supposons le point (a) vrai pour $n(n \geqslant 1)$. Soit $k$ un entier et notons $\left(f^{\prime s}\right)$ la filtration du $\mathbb{F}_{p^{-}}$-espace vectoriel $\left(\mathrm{H}_{\mathbb{Z} / p}\left(\mathrm{~T}_{n} M\right)\right)^{k}$. On a alors un isomorphisme $\left(\mathrm{H}_{\mathbb{Z} / p}\left(\mathrm{~T}_{n} \mathrm{G} \cdot(M)\right)\right)^{k} / f^{\prime 1} \cong \mathrm{H}^{0} \otimes\left(\left(\mathrm{T}_{n} \mathrm{G} \cdot(M)\right) / \mathrm{f}^{1}\right)^{k}$ et une suite exacte

$$
\begin{aligned}
0 \longrightarrow \mathrm{H}^{s} \otimes\left(\left(\mathrm{T}_{n} \mathrm{G}_{\bullet}(M)\right) / \mathrm{f}^{1}\right)^{k-s} & \longrightarrow\left(\mathrm{H}_{\mathbb{Z} / p}\left(\mathrm{~T}_{n} \mathrm{G}_{\bullet}(M)\right)\right)^{k} / f^{\prime s} \\
& \longrightarrow\left(\mathrm{H}_{\mathbb{Z} / p}\left(\mathrm{~T}_{n} \mathrm{G}_{\bullet}(M)\right)\right)^{k} / f^{\prime s-1} \longrightarrow 0
\end{aligned}
$$


pour $s \geqslant 1$ d'où on déduit par récurrence sur $s$ que le $\mathbb{F}_{p}$-espace vectoriel simplicial augmenté $\left(\mathrm{H}_{\mathbb{Z} / p}\left(\mathrm{~T}_{n} \mathrm{G}_{\bullet}(M)\right)\right)^{k} / f^{\prime s} \rightarrow\left(\mathrm{H}_{\mathbb{Z} / p}\left(\mathrm{~T}_{n} M\right)\right)^{k} / f^{\prime s}$ est acyclique puis que la suite exacte ci-dessus induit une suite exacte

$0 \longrightarrow \mathrm{H}^{s} \otimes\left(\left(\mathrm{T}_{n} M\right) / \mathrm{f}^{1}\right)^{k-s} \longrightarrow\left(\mathrm{H}_{\mathbb{Z} / p}\left(\mathrm{~T}_{n} M\right)\right)^{k} / f^{\prime s} \longrightarrow\left(\mathrm{H}_{\mathbb{Z} / p}\left(\mathrm{~T}_{n} M\right)\right)^{k} / f^{\prime s-1} \longrightarrow 0$,

d'où le point $c$. Le point (a) est maintenant conséquence de la proposition 7.3.2 et de l'exactitude du foncteur $\operatorname{Fix}_{\mathcal{U}}$.

Corollaire 7.3.5. - Soit $\pi$ un groupe de Lie compact commutatif; alors

(a) Si $M \in \mathcal{K}_{\mathrm{MU}}$ est libre comme $\widehat{\mathrm{L}}$-algèbre, il en est de même de $\mathrm{T}_{\mathrm{B} \pi} M$ et l'objet simplicial augmenté $\left(\mathrm{T}_{\mathrm{B} \pi} \mathrm{G}_{\bullet}(M)\right) / \mathrm{f}^{1} \rightarrow\left(\mathrm{T}_{\mathrm{B} \pi} M\right) / \mathrm{f}^{1}$ de $\mathcal{E}$ est acyclique.

(b) Soit $M \rightarrow N$ un morphisme entre MU-algèbres instables libres comme $\widehat{\mathrm{L}}$ algèbres. Si $M / \mathrm{f}^{1} \rightarrow N / \mathrm{f}^{1}$ est injectif en chaque degré, il en est de même de $\left(\mathrm{T}_{\mathrm{B} \pi} M\right) / \mathrm{f}^{1} \rightarrow\left(\mathrm{T}_{\mathrm{B} \pi} N\right) / \mathrm{f}^{1}$.

Démonstration. - Pour $\pi=\mathbb{Z} / p^{n}$ avec $n$ fini, le point (a) est déjà démontré. Le point (b) s'obtient par récurrence sur $n$ par le point (c) de la proposition ci-dessus, la proposition 7.3.2 et l'exactitude du foncteur Fix $\mathcal{U}$.

Pour $n=\infty$ on obtient l'acyclicité de $\left(\mathrm{T}_{n} \mathrm{G}_{\bullet}(M)\right) / \mathrm{f}^{1} \rightarrow\left(\mathrm{T}_{n} M\right) / \mathrm{f}^{1}$ et le point (b) par la proposition 7.3.3 et l'exactitude des colimites filtrantes.

Pour $\pi$ quelconque on choisit un morphisme $\mathbb{Z} / p^{n_{1}} \times \cdots \times \mathbb{Z} / p^{n_{r}} \rightarrow \pi$ induisant un isomorphisme entre les cohomologies modulo $p$ des classifiants. On obtient un isomorphisme naturel $\mathrm{T}_{n_{1}} \cdots \mathrm{T}_{n_{r}} \stackrel{\sim}{\longrightarrow} \mathrm{T}_{\mathrm{B} \pi}$ (voir la discussion précédant le théorème 7.1.3). On en déduit le résultat par récurrence sur $r$.

Corollaire 7.3.6. - Soient $\pi$ un groupe de Lie compact commutatif et $M \rightarrow M^{\prime}$ un morphisme entre MU-algèbres instables libres comme $\widehat{\mathrm{L}}$-algèbres. On suppose que l'application induite $M / \mathrm{f}^{1} \rightarrow M^{\prime} / \mathrm{f}^{1}$ est injective en chaque degré, alors tout morphisme de $M \rightarrow \widehat{\mathrm{MU}}^{*} \mathrm{~B} \pi$ dans $\mathcal{K}_{\mathrm{MU}}$ s'étend en un morphisme $M^{\prime} \rightarrow \widehat{\mathrm{MU}}^{*} \mathrm{~B} \pi$.

Démonstration. - Le morphisme induit $\left(\mathrm{T}_{\mathrm{B} \pi} M\right) / \mathrm{f}^{1} \rightarrow\left(\mathrm{T}_{\mathrm{B} \pi} M^{\prime}\right) / \mathrm{f}^{1}$ est injectif en chaque degré par le corollaire ci-dessus.

On dispose des bijections naturelles

$$
\operatorname{Hom}_{\mathcal{K}_{\mathrm{MU}}}\left(\mathrm{T}_{\mathrm{B} \pi} M, \widehat{\mathrm{MU}}^{*}\right) \cong \operatorname{Hom}_{\mathcal{K}_{\mathrm{MU}}}\left(M, \widehat{\mathrm{MU}}^{*} \mathrm{~B} \pi\right)
$$

(voir la section 5.3) et

$$
\operatorname{Hom}_{\mathcal{K}_{\mathrm{MU}}}\left(\mathrm{T}_{\mathrm{B} \pi} M, \widehat{\mathrm{MU}}^{*}\right) \cong \operatorname{Hom}_{\mathcal{K}_{\mathrm{H}}}\left(\left(\mathrm{T}_{\mathrm{B} \pi} M\right) / \mathrm{f}^{1}, \mathbb{Z} / p\right)
$$

(proposition 3.3.1). Comme les éléments en degré 0 de l'algèbre instable $\left(\mathrm{T}_{\mathrm{B} \pi} M\right) / \mathrm{f}^{1}$ s'identifient aux applications continues de l'ensemble profini $\operatorname{Hom}_{\mathcal{K}_{\mathrm{H}}}\left(\left(\mathrm{T}_{\mathrm{B} \pi} M\right) / \mathrm{f}^{1}, \mathbb{Z} / p\right)$ dans $\mathbb{Z} / p$ (voir $[$ La1, $\S 1.8 .1])$, un morphisme $\left(\mathrm{T}_{\mathrm{B} \pi} M\right) / \mathrm{f}^{1} \rightarrow\left(\mathrm{T}_{\mathrm{B} \pi} M^{\prime}\right) / \mathrm{f}^{1}$ injectif degré par degré induit une surjection

$$
\operatorname{Hom}_{\mathcal{K}_{\mathrm{H}}}\left(\left(\mathrm{T}_{\mathrm{B} \pi} M^{\prime}\right) / \mathrm{f}^{1}, \mathbb{Z} / p\right) \longrightarrow \operatorname{Hom}_{\mathcal{K}_{\mathrm{H}}}\left(\left(\mathrm{T}_{\mathrm{B} \pi} M\right) / \mathrm{f}^{1}, \mathbb{Z} / p\right) .
$$


EXemple. - Soient $G$ un groupe de Lie compact connexe et $T$ un tore maximal dans $G$. On suppose que l'homologie entière de $G$ est sans torsion; alors le classifiant $\mathrm{B} G$ est sans $p$-torsion et le morphisme $\mathrm{H}^{*} \mathrm{~B} G \rightarrow \mathrm{H}^{*} \mathrm{~B} T$ est injectif en chaque degré.

Soit $T^{\prime}$ un tore; le corollaire indique que tout morphisme $\widehat{\mathrm{MU}}^{*} \mathrm{~B} G \rightarrow \widehat{\mathrm{MU}}^{*} \mathrm{~B} T^{\prime}$ dans $\mathcal{K}_{\mathrm{MU}}$ se prolonge en un morphisme $\widehat{\mathrm{MU}}^{*} \mathrm{~B} T \rightarrow \widehat{\mathrm{MU}}^{*} \mathrm{~B} T^{\prime}$. On obtient donc une version en MU-théorie, mais avec l'hypothèse $G$ sans torsion, du théorème 4.1 de [Wilk]. Le lien entre la catégorie $\mathcal{K}_{\mathrm{MU}}$ et son analogue en K-théorie est donné dans le cas sans torsion par le théorème d'Hattori Stong ( $c f$. la section 6 de [DL]).

\section{MU-cohomologie du classifiant de $\mathbb{Z} / p^{n}$ et foncteur $\mathrm{T}_{n}$}

Pour $n$ un élément de $\mathbb{N} \cup\{\infty\}$ on note $\mathrm{P}_{n}$ la MU-cohomologie complétée en $p$ du classifiant du groupe $\mathbb{Z} / p^{n}$. (Ce classifiant est un groupe abélien fini simplicial si $n$ est fini.)

Rappelons que si $n$ est fini l'application $\mathrm{B} \mathbb{Z} / p^{n} \rightarrow \mathrm{BS}^{1}$ est à équivalence d'homotopie près le fibré en sphère de la $p^{n}$-puissance tensorielle du fibré linéaire canonique $\eta$ au dessus de $\mathrm{BS}^{1}$. La cofibre homotopique de cette application, qu'on note $\mathrm{Th}_{n}$ (l'espace de Thom du fibré $\eta^{\otimes p^{n}}$ ), est sans $p$-torsion par l'isomorphisme de Thom et de cohomologie modulo $p$ finie en chaque degré.

Notons $x$ la classe d'Euler de $\eta$ en MU-théorie et $\left[p^{n}\right] x$ celle de $\eta^{\otimes p^{n}}\left(x,\left[p^{n}\right] x \in\right.$ $\widehat{\mathrm{MU}}^{2} \mathrm{BS}^{1}$ ); la suite exacte de Gysin appliquée aux espaces projectifs complexes $\mathbb{C P}^{s}$ donne un isomorphisme (de $\widehat{\mathrm{L}}$-algèbres par la proposition 2.2.1)

$$
\mathrm{P}_{\infty} \cong \lim _{s} \widehat{\mathrm{MU}}^{*}[x] / x^{s}=\widehat{\mathrm{MU}}^{*}[[x]]
$$

(l'anneau des séries formelles en $x$ à coefficients dans $\widehat{\mathrm{MU}}^{*}$ ) et une suite exacte de $\widehat{\mathrm{MU}}^{*}$-modules, donc de $\widehat{\mathrm{L}}$-algèbres

$$
0 \longrightarrow \widehat{\mathrm{MU}}^{*}[[x]] \stackrel{-\cdot\left[p^{n}\right] x}{\longrightarrow} \widehat{\mathrm{MU}}^{*}[[x]] \longrightarrow \mathrm{P}_{n} \longrightarrow 0 .
$$

On a donc pour tout entier $s>0$ une suite exacte

$$
0 \longrightarrow \widehat{\mathrm{MU}}^{*}[x] / x^{s-1} \stackrel{-\cdot\left[p^{n}\right] x}{\longrightarrow} \widehat{\mathrm{MU}}^{*}[x] / x^{s} \longrightarrow \mathrm{P}_{n} / \mathrm{F}^{2 s} \longrightarrow 0
$$

de sorte que la $\widehat{\mathrm{L}}$-algèbre quotient $\mathrm{P}_{n} / \mathrm{F}^{s}$ admet une résolution libre de longueur 1 naturelle en $s$. En passant à la limite sur $s$ on obtient que le morphisme $\mathrm{P}_{n} \rightarrow$ $\lim _{s}\left(\mathrm{P}_{n} / \mathrm{F}^{2 s}\right)$ est un isomorphisme. Nous désignerons $\mathrm{P}_{n}$ également par la notation $\mathrm{P}_{n} / \mathrm{F}^{\infty}$.

Nous fixons l'entier $n(n<\infty)$. Nous commençons par établir :

LEMme 8.1. - Soient $M$ une $\widehat{\mathrm{L}}$-algèbre et s un élément de $\mathbb{N} \cup\{\infty\}$; alors pour tout entier $k$ les $\widehat{\mathrm{L}}$-algèbres $\operatorname{Tor}_{k}\left(\mathrm{P}_{n} / \mathrm{F}^{s}, M\right)$ et $\operatorname{Tor}_{k}\left(M, \mathrm{P}_{n} / \mathrm{F}^{s}\right)$ sont isomorphes et pour tout entier $k \geqslant 2$ la $\widehat{\mathrm{L}}$-algèbre $\operatorname{Tor}_{k}\left(\mathrm{P}_{n} / \mathrm{F}^{s}, M\right)$ est nulle. 
Démonstration. - La $\widehat{\mathrm{L}}$-algèbre $\mathrm{P}_{n} / \mathrm{F}^{s}$ est un quotient de la $\widehat{\mathrm{L}}$-algèbre libre $\mathrm{P}_{\infty} / \mathrm{F}^{s}$. Comme $\left(\mathrm{P}_{\infty} / \mathrm{F}^{s}\right) / \mathrm{f}^{1}$ est fini en chaque degré, la $\widehat{\mathrm{L}}$-algèbre $\operatorname{Tor}_{k}\left(N, \mathrm{P}_{\infty} / \mathrm{F}^{s}\right)$ est nulle pour tout entier $k \geqslant 1$ et toute $\widehat{\mathrm{L}}$-algèbre $N$ par le corollaire 4.1.24.

Soit $M_{*} \rightarrow M$ une résolution libre de $M$. Comme $\mathrm{P}_{n} / \mathrm{F}^{s}$ admet une résolution libre de longueur 1 la $\widehat{\mathrm{L}}$-algèbre $\operatorname{Tor}_{k}\left(\mathrm{P}_{n} / \mathrm{F}^{s}, M\right)$ est nulle pour tout $k \geqslant 2$ et $\operatorname{Tor}_{1}\left(\mathrm{P}_{n}, M_{k}\right)$ est nulle pour tout entier $k$ par le lemme 4.1.21. On peut maintenant invoquer le corollaire B.5 pour conclure.

Les deux propositions qui suivent sont des reformulations des résultats de Landweber ([Lan]) dont nous reprenons les arguments. Comparer aussi avec la section 9 de $[\mathbf{R W Y}]$ pour la formule de Künneth.

Proposition 8.2. - Soit $M$ une $\widehat{\mathrm{L}}$-algèbre isomorphe à l'image par $\mathrm{M}_{k}^{\infty}$ d'une $\widehat{\mathrm{L}}_{k}$-algèbre pour un certain entier $k$; alors la tour de $\widehat{\mathrm{L}}$-algèbres $\left(\operatorname{Tor}_{1}\left(\mathrm{P}_{n} / \mathrm{F}^{s}, M\right)\right)_{s}$ est pro-triviale. En particulier la $\widehat{\mathrm{L}}$-algèbre $\operatorname{Tor}_{1}\left(\mathrm{P}_{n}, M\right)$ est nulle et le morphisme $\mathrm{P}_{n} \widehat{\otimes} M \rightarrow \lim _{s}\left(\mathrm{P}_{n} / \mathrm{F}^{s} \widehat{\otimes} M\right)$ est un isomorphisme.

(Voir la section 4.1 pour la définition de $\Lambda_{k}, \widehat{\mathrm{L}}_{k}$ et $\mathrm{M}_{k}^{\infty}$.)

Démonstration. - La série formelle image de $\left[p^{n}\right] x$ par le morphisme entre anneaux de coefficients $\widehat{\mathrm{MU}}^{*} \rightarrow \widehat{\mathrm{MU}}^{*} \otimes_{\Lambda_{k}}\left(\Lambda_{k} / \mathrm{f}^{1}\right) \cong \mathrm{MU}^{*} /\left(p, x_{1}, \ldots, x_{k}\right)$ n'est pas nulle : voir les lemmes 4 et 5 de [Lan] et la proposition 4.3 de [tD]; soit $i$ sa valuation. Pour $s \geqslant i$ notons $K_{s}$, respectivement $L_{s}$, le sous- $\widehat{M U}^{*}$-module (ou de façon équivalente la sous- $\widehat{\mathrm{L}}$-algèbre) de la $\widehat{\mathrm{MU}}^{*}$-algèbre polynomiale tronquée $\widehat{\mathrm{MU}}^{*}[x] / x^{s-1}$ engendré par les éléments $x^{l}, s-i \leqslant l \leqslant s-1$, respectivement par les éléments $x^{l}, 0 \leqslant l<s-i$. La $\widehat{\mathrm{L}}$-algèbre $\widehat{\mathrm{MU}}^{*}[x] / x^{s-1}$ s'écrit comme la somme directe de $K_{s}$ et de $L_{s}$ naturellement en $s$ de sorte qu'on a une suite exacte naturelle :

$$
0 \longrightarrow K_{s} \longrightarrow \operatorname{Coker}\left(-\cdot\left[p^{n}\right] x: L_{s} \rightarrow \widehat{\mathrm{MU}}^{*}[x] / x^{s}\right) \longrightarrow \mathrm{P}_{n} / \mathrm{F}^{2 s} \longrightarrow 0 .
$$

On note $\mathrm{O}_{k} N$ la $\widehat{\mathrm{L}}_{k}$-algèbre sous-jacente à une $\widehat{\mathrm{L}}$-algèbre $N$. La multiplication par $\left[p^{n}\right] x$ induit un morphisme injectif $\mathrm{O}_{k} L_{s} \otimes_{\widehat{\mathrm{L}}_{k}} \Lambda_{k} / \mathrm{f}^{1} \rightarrow \mathrm{O}_{k}\left(\widehat{\mathrm{MU}}^{*}[x] / x^{s}\right) \otimes_{\widehat{\mathrm{L}}_{k}} \Lambda_{k} / \mathrm{f}^{1}$. Autrement dit, puisque les $\widehat{\mathrm{L}}_{k}$-algèbres sous-jacentes à $L_{s}$ et $\widehat{\mathrm{MU}}^{*}[x] / x^{s}$ sont libres (lemme 4.1.17), la $\widehat{\mathrm{L}}_{k}$-algèbre $\operatorname{Tor}_{1}^{\widehat{\mathrm{L}}_{k}}\left(\mathrm{O}_{k} \operatorname{Coker}\left(L_{s} \rightarrow \widehat{\mathrm{MU}}^{*}[x] / x^{s}\right), \Lambda_{k} / \mathrm{f}^{1}\right)$ est nulle donc la $\widehat{\mathrm{L}}_{k}$-algèbre sous-jacente à $\operatorname{Coker}\left(L_{s} \rightarrow \widehat{\mathrm{MU}}^{*}[x] / x^{s}\right)$ est libre par la proposition 4.1.25. On en déduit que pour toute $\widehat{\mathrm{L}}_{k}$-algèbre $N$, la $\widehat{\mathrm{L}}_{k}$-algèbre $\operatorname{Tor}_{1}^{\widehat{\mathrm{L}}_{k}}\left(\mathrm{O}_{k}\left(\mathrm{P}_{n} / \mathrm{F}^{2 s}\right), N\right)$ est le noyau du morphisme

$$
\left(\mathrm{O}_{k} K_{s}\right) \otimes_{\widehat{\mathrm{L}}_{k}} N \longrightarrow\left(\mathrm{O}_{k} \operatorname{Coker}\left(L_{s} \rightarrow \widehat{\mathrm{MU}}^{*}[x] / x^{s}\right)\right) \otimes_{\widehat{\mathrm{L}}_{k}} N .
$$

On observe maintenant que le morphisme $K_{s+i} \rightarrow K_{s}$ est nul quel que soit $s$, donc la tour de $\widehat{\mathrm{L}}_{k}$-algèbres $\left(\left(\mathrm{O}_{k} K_{s}\right) \otimes_{\widehat{\mathrm{L}}_{k}} N\right)_{s}$ est pro-triviale, donc également le sous-objet $\left(\operatorname{Tor}_{1}^{\widehat{\mathrm{L}}_{k}}\left(\mathrm{O}_{k}\left(\mathrm{P}_{n} / \mathrm{F}^{2 s}\right), N\right)\right)_{s}$. L'isomorphisme

$$
\mathrm{O}_{k} \operatorname{Tor}_{1}\left(\mathrm{P}_{n} / \mathrm{F}^{2 s}, \mathrm{M}_{k}^{\infty}(N)\right) \cong \operatorname{Tor}_{1}^{\widehat{\mathrm{L}}_{k}}\left(\mathrm{O}_{k}\left(\mathrm{P}_{n} / \mathrm{F}^{2 s}\right), N\right)
$$


donné par le point (a) de la proposition 4.1.23 et le lemme 8.1 montre que la tour $\left(\operatorname{Tor}_{1}\left(\mathrm{P}_{n} / \mathrm{F}^{s}, M\right)\right)_{s}$ est pro-triviale.

Les deux autres affirmations de la proposition sont maintenant des conséquences de la proposition 4.1.31 : les morphismes $\operatorname{Tor}_{1}\left(M, \mathrm{P}_{n}\right) \rightarrow \lim _{s} \operatorname{Tor}_{1}\left(M, \mathrm{P}_{n} / \mathrm{F}^{s}\right)$ et $M \widehat{\otimes} \mathrm{P}_{n} \rightarrow \lim _{s}\left(M \widehat{\otimes} \mathrm{P}_{n} / \mathrm{F}^{s}\right)$ sont des isomorphismes par son point (b) au vu des isomorphismes $\operatorname{Tor}_{1}\left(M, \mathrm{P}_{n}\right) \cong \operatorname{Tor}_{1}\left(\mathrm{P}_{n}, M\right)$ et $\operatorname{Tor}_{1}\left(M, \mathrm{P}_{n} / \mathrm{F}^{s}\right) \cong \operatorname{Tor}_{1}\left(\mathrm{P}_{n} / \mathrm{F}^{s}, M\right)$ donnés par le lemme 8.1.

Remarquons que le $\widehat{\mathrm{MU}}^{*} / \mathrm{f}^{1}$-module $\operatorname{Tor}_{1}\left(\mathrm{P}_{n}, \widehat{\mathrm{MU}}^{*} / \mathrm{f}^{1}\right)$ s'identifie à la partie impaire de la cohomologie modulo $p$ de $\mathrm{B} \mathbb{Z} / p^{n}$ : la $\widehat{\mathrm{L}}$-algèbre $\mathrm{P}_{n}$ n'est pas libre.

Proposition 8.3. - Soit $n$ un entier.

(a) Soit $\left(M_{s}\right)$ une tour de MU-algèbres instables nulles en degré assez grand; alors la $\widehat{\mathrm{L}}$-algèbre $\operatorname{Tor}_{1}\left(\mathrm{P}_{n}, \lim _{s} M_{s}\right)$ est nulle et on a une suite exacte

$$
0 \longrightarrow \mathrm{P}_{n} \widehat{\otimes} \lim _{s} M_{s} \longrightarrow \lim _{s}\left(\mathrm{P}_{n} \widehat{\otimes} M_{s}\right) \longrightarrow \operatorname{Tor}_{1}\left(\mathrm{P}_{n}, \lim _{s}^{1} M_{s}\right) \longrightarrow 0 .
$$

(b) Soit $X$ un espace profini dont la MU-cohomologie continue est complète pour la filtration squelettale; alors le morphisme $\mathrm{P}_{n} \widehat{\otimes} \widehat{\mathrm{MU}}^{*} X \rightarrow \widehat{\mathrm{MU}}^{*}\left(\mathrm{BZ} / p^{n} \times X\right)$ est un isomorphisme.

Démonstration. - Soient $k$ un entier et $M$ une MU-algèbre instable nulle en degré supérieur ou égal à $k$, alors $M$ est le coégalisateur du 1-complexe

$$
\mathrm{G}^{2}(M) / \mathrm{F}^{k} \rightleftarrows \mathrm{G}(M) / \mathrm{F}^{k} .
$$

Les $\widehat{\mathrm{L}}$-algèbres $\mathrm{G}^{2}(M) / \mathrm{F}^{k}$ et $\mathrm{G}(M) / \mathrm{F}^{k}$ sont libres sur des ensembles gradués concentrés en degrés compris entre 0 et $k-1\left(\mathrm{G}(M) / \mathrm{f}^{1}\right.$ est la cohomologie modulo $p$ continue d'un espace profini) donc $M$ est de présentation en degré borné donc dans l'image de $\mathrm{M}_{l}^{\infty}$ pour un certain entier $l$. On en déduit par la proposition 8.2 que la $\widehat{\mathrm{L}}$-algèbre $\operatorname{Tor}_{1}\left(\mathrm{P}_{n}, M\right)$ est nulle.

Soit maintenant $\left(M_{s}\right)$ une tour de MU-algèbres instables nulles en degré assez grand et notons $M_{\infty}$ sa limite. Les $\widehat{\mathrm{L}}$-algèbres $\operatorname{Tor}_{k}\left(\mathrm{P}_{n}, M_{s}\right)$ sont nulles pour $k=1$ par la proposition 8.2 et pour $k \geqslant 2$ parce que $\mathrm{P}_{n}$ admet une résolution libre de longueur 1 . Le point (a) est alors la conséquence du point (c) de la proposition 4.1.31.

Pour le point (b) observons que les complétés profinis de $\mathrm{BS}^{1}$ et $\mathrm{Th}_{n}$ sont sans $p$ torsion, de cohomologie modulo $p$ finie en chaque degré et forment une MU-résolution libre de longueur finie de $\mathrm{B} \mathbb{Z} / p^{n}$ dans $\widehat{\mathcal{S}}_{\mathrm{pt}}$. Pour tout espace profini $X$ le morphisme $\widehat{\mathrm{MU}}^{*} X \widehat{\otimes} \widehat{\mathrm{MU}}^{*} Y \rightarrow \widehat{\mathrm{MU}}^{*}(X \times Y)$ est un isomorphisme si $Y$ est le complété profini de $\mathrm{BS}^{1}$ ou de $\mathrm{Th}_{n}$ par la proposition 4.3.2. On en déduit avec la proposition 4.3.1 que le morphisme $\mathrm{P}_{n} \widehat{\otimes} \widehat{\mathrm{MU}}^{*} X \rightarrow \widehat{\mathrm{MU}}^{*}\left(\mathrm{BZ} / p^{n} \times X\right)$ est un isomorphisme pour tout espace profini $X$ tel que $\operatorname{Tor}_{1}\left(\mathrm{P}_{n}, \widehat{\mathrm{MU}}^{*} X\right)$ est nul. 
On a équivalence entre les conditions $\widehat{\mathrm{MU}}^{*} X \rightarrow \lim _{s} \widehat{\mathrm{MU}}^{*} \mathrm{Sk}_{s} X$ est un isomorphisme et $\lim _{s}^{1} \widehat{\mathrm{MU}}^{*} \mathrm{Sk}_{s} X$ est nul par la proposition 1.6.2. Si ces conditions sont vérifiées alors la $\widehat{\mathrm{L}}$-algèbre $\operatorname{Tor}_{1}\left(\mathrm{P}_{n}, \widehat{\mathrm{MU}}^{*} X\right)$ est nulle par le point $(\mathrm{a})$, d'où le résultat.

Remarque. - Soit $X$ un espace profini quelconque. On dispose de la suite exacte de Milnor

$$
0 \longrightarrow \lim _{s}^{1} \Sigma \widehat{\mathrm{MU}}^{*} \mathrm{Sk}_{s} X \longrightarrow \widehat{\mathrm{MU}}^{*} X \longrightarrow \lim _{s} \widehat{\mathrm{MU}}^{*} \mathrm{Sk}_{s} X \longrightarrow 0
$$

laquelle induit par la proposition B.4 la suite exacte courte

$$
\begin{aligned}
0 \longrightarrow \operatorname{Tor}_{1}\left(\mathrm{P}_{n}, \lim _{s}^{1} \Sigma \widehat{\mathrm{MU}}^{*} \operatorname{Sk}_{s} X\right) \\
\longrightarrow \operatorname{Tor}_{1}\left(\mathrm{P}_{n}, \widehat{\mathrm{MU}}^{*} X\right) \longrightarrow \operatorname{Tor}_{1}\left(\mathrm{P}_{n}, \lim _{s} \widehat{\mathrm{MU}}^{*} \operatorname{Sk}_{s} X\right) \longrightarrow 0
\end{aligned}
$$

La $\widehat{\mathrm{L}}$-algèbre $\operatorname{Tor}_{1}\left(\mathrm{P}_{n}, \lim _{s} \widehat{\mathrm{MU}}^{*} \mathrm{Sk}_{s} X\right)$ est nulle par le point (a) de la proposition ci-dessus. On n'en déduit pas cependant un isomorphisme $\mathrm{P}_{n} \widehat{\otimes} \widehat{M U}^{*} X \cong$ $\widehat{\mathrm{MU}}^{*}\left(\mathrm{~B} \mathbb{Z} / p^{n} \times X\right)$ : nous ne savons pas que la $\widehat{\mathrm{L}}$-algèbre $\operatorname{Tor}_{1}\left(\mathrm{P}_{n}, \lim _{s}^{1} \Sigma \widehat{\mathrm{MU}}^{*} \mathrm{Sk}_{s} X\right)$ est nulle sans hypothèse sur $X$.

On note $\mathcal{K}_{\mathrm{MU}}{ }^{0}$ la sous-catégorie pleine de $\mathcal{K}_{\mathrm{MU}}$ formée des MU-algèbres instables nulles en degré assez grand et, pour $s$ un entier strictement positif, $\mathcal{K}_{\mathrm{MU}}{ }^{<s}$ celle formée des MU-algèbres instables nulles en degré supérieur ou égal à $s$.

Proposition 8.4. - Soit $S$ un ensemble gradué; le foncteur $\mathcal{K}_{\mathrm{MU}}{ }^{<s} \rightarrow \mathcal{E}$ ns-gr, $N \mapsto$ $\operatorname{Hom}_{\mathcal{K}_{\mathrm{MU}}}\left(\mathrm{G}(S), \mathrm{P}_{n} \widehat{\otimes} N\right)$ est représentable.

Démonstration. - On utilise le critère de représentabilité de Freyd (cf. [Mac, chap. V, $\S 6])$ :

Puisque $\mathrm{P}_{n}$ admet une présentation par des $\widehat{\mathrm{L}}$-algèbres libres sur un ensemble gradué fini en chaque degré, le foncteur $\mathcal{K}_{\mathrm{MU}} \rightarrow \mathcal{K}_{\mathrm{MU}}, N \mapsto \mathrm{P}_{n} \widehat{\otimes} N$ commute aux produits indexés par un ensemble par le lemme 4.1.30.

Soient $\mathrm{d}^{0}, \mathrm{~d}^{1}: M^{0} \rightrightarrows M^{1}$ un diagramme de $\mathcal{K}_{\mathrm{MU}}{ }^{<s}$ et $M$ son égalisateur. Puisque l'oubli $\mathcal{K}_{\mathrm{MU}} \rightarrow \widehat{\mathcal{M}}$ commute aux limites, la $\widehat{\mathrm{L}}$-algèbre sous-jacente à $M$ est le noyau du morphisme $\mathrm{d}^{0}-\mathrm{d}^{1}: M^{0} \rightarrow M^{1}$. Notons $Q$ le conoyau dans $\widehat{\mathcal{M}} \mathrm{du}$ morphisme $M \rightarrow M^{0}$ et $R$ celui de $\mathrm{d}^{0}-\mathrm{d}^{1}: M^{0} \rightarrow M^{1}$. Puisque $M, M^{0}$ et $M^{1}$ sont des MUalgèbres instables nulles en degré supérieur ou égal à $s$, les $\widehat{L}$-algèbres sous-jacentes à $M, M^{0}$ et $M^{1}$ sont de présentation en degré borné donc les $\widehat{L}$-algèbres $Q$ et $R$ sont isomorphes à l'image par $\mathrm{M}_{k}^{\infty}$ de $\widehat{\mathrm{L}}_{k}$-algèbres pour un entier $k$ assez grand par la proposition 4.1.27. On en déduit par la proposition 8.2 que les $\widehat{\mathrm{L}}$-algèbres $\operatorname{Tor}_{1}\left(Q, \mathrm{P}_{n}\right)$ et $\operatorname{Tor}_{1}\left(R, \mathrm{P}_{n}\right)$ sont nulles, donc que les suites

$$
0 \longrightarrow \mathrm{P}_{n} \widehat{\otimes} M \longrightarrow \mathrm{P}_{n} \widehat{\otimes} M^{0} \longrightarrow \mathrm{P}_{n} \widehat{\otimes} Q \longrightarrow 0
$$

et

$$
0 \longrightarrow \mathrm{P}_{n} \widehat{\otimes} Q \longrightarrow \mathrm{P}_{n} \widehat{\otimes} M^{1} \longrightarrow \mathrm{P}_{n} \widehat{\otimes} R \longrightarrow 0
$$


sont exactes. On en déduit que le diagramme

$$
\mathrm{P}_{n} \widehat{\otimes} M \longrightarrow \mathrm{P}_{n} \widehat{\otimes} M^{0} \longrightarrow \mathrm{P}_{n} \widehat{\otimes} M^{1}
$$

est égalisateur dans $\mathcal{K}_{\mathrm{MU}}$.

Comme les limites indexées par une catégorie petite s'expriment en terme de produits indexés par un ensemble et d'un égalisateur, le foncteur $\mathcal{K}_{\mathrm{MU}}{ }^{<s} \rightarrow \mathcal{K}_{\mathrm{MU}}$, $N \mapsto \mathrm{P}_{n} \widehat{\otimes} N$ commute aux limites. On en déduit que le foncteur $\mathcal{K}_{\mathrm{MU}}{ }^{<s} \rightarrow \mathcal{E}$ ns-gr, $N \mapsto \operatorname{Hom}_{\mathcal{K}_{\mathrm{MU}}}\left(\mathrm{G}(S), \mathrm{P}_{n} \widehat{\otimes} N\right)$ commute aux limites.

Il reste à montrer l'existence d'un ensemble $T$ d'objets de $\mathcal{K}_{\mathrm{MU}}{ }^{<s}$ tel que tout morphisme $f: \mathrm{G}(S) \rightarrow \mathrm{P}_{n} \widehat{\otimes} N$ dans $\mathcal{K}_{\mathrm{MU}}$ s'écrive comme la composée $\mathrm{G}(S) \rightarrow$ $\mathrm{P}_{n} \widehat{\otimes} M \rightarrow \mathrm{P}_{n} \widehat{\otimes} N$ pour un objet $M \in T$ et des morphismes $\mathrm{G}(S) \rightarrow \mathrm{P}_{n} \widehat{\otimes} M$ et $M \rightarrow N$ dans $\mathcal{K}_{\mathrm{MU}}$.

Observons d'abord que tout morphisme $\mathrm{G}(S) \rightarrow \mathrm{P}_{n} \widehat{\otimes} N$ se relève en un morphisme $\mathrm{G}(S) \rightarrow \mathrm{P}_{\infty} \widehat{\otimes} N$ dans $\mathcal{K}_{\mathrm{MU}}:$ Cela vient de ce que $P_{\infty} \rightarrow \mathrm{P}_{n}$ est un épimorphisme dans $\widehat{\mathcal{M}}$, de l'exactitude à droite du produit tensoriel dans $\widehat{\mathcal{M}}$, de ce que les épimorphismes dans $\widehat{\mathcal{M}}$ sont épi dans $\mathcal{E}$ ns-gr et de ce que $\mathrm{G}(S)$ est un objet libre sur un ensemble gradué.

Puisque la $\widehat{\mathrm{L}}$-algèbre sous-jacente à $\mathrm{P}_{\infty}$ est libre sur un ensemble fini en chaque degré, le foncteur $\mathrm{P}_{\infty} \widehat{\otimes}-$ admet un adjoint à gauche $\left(-: \mathrm{P}_{\infty}\right)_{\mathcal{K}_{\mathrm{MU}}}$ d'après la section 5.2. Le morphisme $\mathrm{G}(S) \rightarrow \mathrm{P}_{\infty} \widehat{\otimes} N$ s'écrit donc comme la composée de l'unité d'adjonction $\mathrm{G}(S) \rightarrow \mathrm{P}_{\infty} \widehat{\otimes}\left(\mathrm{G}(S): \mathrm{P}_{\infty}\right) \mathcal{K}_{\mathrm{MU}}$ et du morphisme

$$
\mathrm{P}_{\infty} \widehat{\otimes}\left(\mathrm{G}(S): \mathrm{P}_{\infty}\right) \mathcal{K}_{\mathrm{MU}} \longrightarrow \mathrm{P}_{\infty} \widehat{\otimes} N
$$

induit par un morphisme $\left(\mathrm{G}(S): \mathrm{P}_{\infty}\right) \mathcal{K}_{\mathrm{MU}} \rightarrow N$ de $\mathcal{K}_{\mathrm{MU}}$.

Enfin comme $N$ est nulle en degré supérieur ou égal à $s$, le morphisme $\left(\mathrm{G}(S): \mathrm{P}_{\infty}\right)_{\mathcal{K}_{\mathrm{MU}}} \rightarrow N$ se factorise par la G-algèbre quotient $\left(\mathrm{G}(S): \mathrm{P}_{\infty}\right)_{\mathcal{K}_{\mathrm{MU}}} / \mathrm{F}^{s}$ (voir la section 3.1). Conclusion : le morphisme $\mathrm{G}(S) \rightarrow \mathrm{P}_{n} \widehat{\otimes} N$ s'écrit comme la composée

$$
\begin{aligned}
\mathrm{G}(S) \longrightarrow \mathrm{P}_{\infty} \widehat{\otimes}\left(\mathrm{G}(S): \mathrm{P}_{\infty}\right) \mathcal{K}_{\mathrm{MU}} \longrightarrow \mathrm{P}_{\infty} \widehat{\otimes}\left(\left(\mathrm{G}(S): \mathrm{P}_{\infty}\right) \mathcal{K}_{\mathrm{MU}} / \mathrm{F}^{s}\right) \\
\longrightarrow \mathrm{P}_{n} \widehat{\otimes}\left(\left(\mathrm{G}(S): \mathrm{P}_{\infty}\right)_{\mathcal{K}_{\mathrm{MU}}} / \mathrm{F}^{s}\right) \longrightarrow \mathrm{P}_{n} \widehat{\otimes} N
\end{aligned}
$$

On peut donc prendre pour $T$ l'ensemble réduit au seul élément $\left(\mathrm{G}(S): \mathrm{P}_{\infty}\right) \mathcal{K}_{\mathrm{MU}} / \mathrm{F}^{s}$.

Notons $G_{s} \in \mathcal{K}_{\mathrm{MU}}{ }^{<s}$ l'objet représentant le foncteur $N \mapsto \operatorname{Hom}_{\mathcal{K}_{\mathrm{MU}}}\left(\mathrm{G}(S), \mathrm{P}_{n} \widehat{\otimes} N\right)$. Comme le foncteur $\mathrm{P}_{n} \widehat{\otimes}-$ transforme les épimorphismes de $\widehat{\mathcal{M}}$ en épimorphismes de $\widehat{\mathcal{M}}$, le foncteur $N \mapsto \operatorname{Hom}_{\mathcal{K}_{\mathrm{MU}}}<{ }\left(G_{s}, N\right)$ transforme les morphismes épis dans $\widehat{\mathcal{M}}$ en surjections. En particulier le morphisme $\mathrm{G}\left(G_{s}\right) / \mathrm{F}^{s} \rightarrow G_{s}$ induit par la structure de G-algèbre de $G_{s}$ admet une section dans $\mathcal{K}_{\mathrm{MU}}$.

L'inclusion $\mathcal{K}_{\mathrm{MU}}{ }^{<s} \subset \mathcal{K}_{\mathrm{MU}}{ }^{<+1}$ induit un morphisme $G_{s+1} \rightarrow G_{s}$ et un isomorphisme $\operatorname{colim}_{s} \operatorname{Hom}_{\mathcal{K}_{\mathrm{MU}}}\left(G_{s}, N\right) \cong \operatorname{Hom}_{\mathcal{K}_{\mathrm{MU}}}\left(\mathrm{G}(S), \mathrm{P}_{n} \widehat{\otimes} N\right)$ pour tout $N \in \mathcal{K}_{\mathrm{MU}}{ }^{0}$. Notons $G_{\infty}$ la limite de la tour $\operatorname{des} G_{s}$. 
LEMME 8.5. - La $\widehat{\mathrm{L}}$-algèbre sous-jacente à $G_{\infty}$ est dans $\widehat{\mathcal{L}}$ et le morphisme $\mathrm{G}_{\infty} \rightarrow \mathrm{G}_{s}$ induit un isomorphisme $G_{\infty} / \mathrm{F}^{s} \rightarrow G_{s}$ dans $\mathcal{K}_{\mathrm{MU}}$.

Démonstration. - On a pour tout objet $N$ de $\mathcal{K}_{\mathrm{MU}}{ }^{<s}$ des bijections naturelles

$$
\begin{aligned}
\operatorname{Hom}_{\mathcal{K}_{\mathrm{MU}}}\left(G_{s+1} / \mathrm{F}^{s}, N\right) \cong \operatorname{Hom}_{\mathcal{K}_{\mathrm{MU}}}\left(G_{s+1}, N\right) & \cong \operatorname{Hom}_{\mathcal{K}_{\mathrm{MU}}}\left(\mathrm{G}(S), \mathrm{P}_{n} \widehat{\otimes} N\right) \\
& \cong \operatorname{Hom}_{\mathcal{K}_{\mathrm{MU}}}\left(G_{s}, N\right) .
\end{aligned}
$$

Comme $G_{s+1} / \mathrm{F}^{s}$ et $G_{s}$ sont tous deux dans $\mathcal{K}_{\mathrm{MU}}{ }^{<s}$, on en déduit qu'ils sont isomorphes. En particulier la tour $\left(G_{s} / \mathrm{f}^{1}\right)_{s}$ est stationnaire en chaque degré. Comme $G_{s}$ est un facteur direct de $\mathrm{G}\left(G_{s}\right) / \mathrm{F}^{s}$, la $\widehat{\mathrm{L}}$-algèbre sous-jacente à $G_{s}$ est dans $\widehat{\mathcal{L}}$. On déduit alors du lemme 2.1.12 que la $\widehat{\mathrm{L}}$-algèbre sous-jacente à $G_{\infty}$ est dans $\widehat{\mathcal{L}}$ et que l'application $G_{\infty} / \mathrm{f}^{1} \rightarrow \lim _{s}\left(G_{s} / \mathrm{f}^{1}\right)$ est un isomorphisme. On en déduit ensuite que le quotient $G_{\infty} / \mathrm{F}^{s}$ est dans $\widehat{\mathcal{L}}$ et que le morphisme $G_{\infty} / \mathrm{F}^{s} \rightarrow G_{s}$ est un isomorphisme modulo $\mathrm{f}^{1}$ donc un isomorphisme dans $\widehat{\mathcal{L}}$ par le corollaire 2.1.4.

On fixe pour tout entier $s$ une section du morphisme $\mathrm{G}\left(G_{s}\right) / \mathrm{F}^{s} \rightarrow G_{s}$ et on définit un morphisme $\mathrm{G}\left(G_{s+1}\right) / \mathrm{F}^{s+1} \rightarrow \mathrm{G}\left(G_{s}\right) / \mathrm{F}^{s}$ comme la composée

$$
\mathrm{G}\left(G_{s+1}\right) / \mathrm{F}^{s+1} \longrightarrow G_{s+1} \longrightarrow G_{s} \longrightarrow \mathrm{G}\left(G_{s}\right) / \mathrm{F}^{s} \text {. }
$$

On obtient un pro-isomorphisme entre les tours d'objets $\left(\mathrm{G}\left(G_{s}\right) / \mathrm{F}^{s}\right)_{s}$ et $\left(G_{s}\right)_{s}$ de $\mathcal{K}_{\mathrm{MU}}{ }^{0}$.

On note ind-h $\widehat{\mathcal{S}}^{0}$ la catégorie ayant pour objets les diagrammes dans h $\widehat{\mathcal{S}}$ d'espaces profinis de dimension finie indexés par une catégorie petite cofiltrante et pour morphismes entre deux tels objets $X(-)$ et $Y(-)$ les éléments de l'ensemble

$$
\lim _{j} \operatorname{colim}_{i} \operatorname{Hom}_{\mathrm{h} \widehat{\mathcal{S}}}(X(j), Y(i)) .
$$

A tout espace profini $X$ correspond l'objet $\left(\operatorname{Sk}_{s} X\right)_{s}$ de ind-h $\widehat{\mathcal{S}}^{0}$. Si $Z$ est un espace profini de dimension finie, l'application

$$
\operatorname{colim}_{s} \operatorname{Hom}_{\mathrm{h} \widehat{\mathcal{S}}}\left(Z, \mathrm{Sk}_{s} X\right) \longrightarrow \operatorname{Hom}_{\mathrm{h} \widehat{\mathcal{S}}}(Z, X)
$$

est une bijection par le lemme 1.4.1.

LEMME 8.6. - Soit $X(-)$ un objet de ind-h $\widehat{\mathcal{S}}^{0}$. Le foncteur $Y \mapsto \widehat{\mathrm{MU}}^{*} Y$ induit une bijection

$$
\operatorname{Hom}_{\text {ind-h } \widehat{\mathcal{S}}^{0}}\left(X(-),\left(\mathrm{Sk}_{s-1} \mathrm{~K}\left(G_{s}\right)\right)_{s}\right) \stackrel{\sim}{\longrightarrow} \operatorname{Hom}_{\text {pro- } \mathcal{K}_{\mathrm{MU}}}\left(\mathrm{G}\left(G_{s}\right) / \mathrm{F}^{s}, \widehat{\mathrm{MU}}^{*} X(-)\right) .
$$

Démonstration. - Il suffit de considérer le cas où $X(-)$ est un objet constant. Soit donc $X$ un espace profini de dimension finie. On dispose des bijections naturelles

$$
\begin{aligned}
\operatorname{Hom}_{\mathrm{ind}-\mathrm{h} \widehat{\mathcal{S}}^{0}}\left(X,\left(\operatorname{Sk}_{s-1} \mathrm{~K}\left(G_{s}\right)\right)_{s}\right) & =\operatorname{colim}_{s} \operatorname{Hom}_{\mathrm{h} \widehat{\mathcal{S}}}\left(X, \operatorname{Sk}_{s-1} \mathrm{~K}\left(G_{s}\right)\right) \\
& \cong \operatorname{colim}_{s} \operatorname{Hom}_{\mathrm{h} \widehat{\mathcal{S}}}\left(X, \mathrm{~K}\left(G_{s}\right)\right) \\
& \cong \operatorname{colim}_{s} \operatorname{Hom}_{\mathcal{K}_{\mathrm{MU}}}\left(\mathrm{G}\left(G_{s}\right), \widehat{\mathrm{MU}}^{*} X\right) \\
& \cong \operatorname{colim}_{s} \operatorname{Hom}_{\mathcal{K}_{\mathrm{MU}}}\left(\mathrm{G}\left(G_{s}\right) / \mathrm{F}^{s}, \widehat{\mathrm{MU}}^{*} X\right),
\end{aligned}
$$

la dernière bijection venant de ce que $\widehat{\mathrm{MU}}^{*} X$ est nul en degré assez grand. 
Proposition 8.7. - On a un isomorphisme

$$
\left(\mathrm{Sk}_{s-1} \mathrm{~K}\left(G_{s}\right)\right)_{s} \cong\left(\mathrm{Sk}_{s} \operatorname{hom}\left(\mathrm{B} \mathbb{Z} / p^{n}, \mathrm{~K}(S)\right)\right)_{s}
$$

dans ind-h $\widehat{\mathcal{S}}^{0}$ et un isomorphisme

$$
\mathrm{T}_{n} \mathrm{G}(S) \cong G_{\infty}
$$

dans $\mathcal{K}_{\mathrm{MU}}$.

Démonstration. - On a pour tout espace profini $Z$ de dimension finie un isomorphisme $\mathrm{P}_{n} \widehat{\otimes} \widehat{\mathrm{MU}}^{*} Z \cong \widehat{\mathrm{MU}}^{*}\left(\mathrm{~B} \mathbb{Z} / p^{n} \times Z\right)$ par le point (b) de la proposition 8.3 donc, avec ce qui précède, des bijections naturelles

$$
\begin{aligned}
\operatorname{Hom}_{\text {ind-h } \widehat{\mathcal{S}}^{0}}\left(Z,\left(\operatorname{Sk}_{s} \operatorname{hom}\left(\mathrm{BZ} / p^{n}, \mathrm{~K}(S)\right)\right)_{s}\right) & \cong \operatorname{Hom}_{\mathrm{h} \widehat{\mathcal{S}}}\left(Z, \operatorname{hom}\left(\mathrm{BZ} / p^{n}, \mathrm{~K}(S)\right)\right. \\
& \cong \operatorname{Hom}_{\mathcal{K}_{\mathrm{MU}}}\left(\mathrm{G}(S), \mathrm{P}_{n} \widehat{\otimes} \widehat{\mathrm{MU}}^{*} Z\right) \\
& \cong \operatorname{colim}_{s} \operatorname{Hom}_{\mathcal{K}_{\mathrm{MU}}}\left(\mathrm{G}\left(G_{s}\right) / \mathrm{F}^{s}, \widehat{\mathrm{MU}}^{*} Z\right) \\
& \cong \operatorname{Hom}_{\text {ind-h }} \widehat{\mathcal{S}}^{0}\left(Z,\left(\operatorname{Sk}_{s-1} \mathrm{~K}\left(G_{s}\right)\right)_{s}\right)
\end{aligned}
$$

d'où le premier isomorphisme. Celui-ci induit en MU-cohomologie continue un proisomorphisme entre tours de $\mathcal{K}_{\mathrm{MU}}{ }^{0}$.

Comme les espaces profinis $\mathrm{K}\left(G_{s}\right)$ et $\operatorname{hom}\left(\mathrm{B} \mathbb{Z} / p^{n}, \mathrm{~K}(S)\right)$ sont sans $p$-torsion, le second par le point (a) de la proposition 6.3 ou par le lemme ci-dessous, les morphismes $\mathrm{G}\left(G_{s}\right) / \mathrm{F}^{s} \rightarrow \widehat{\mathrm{MU}}^{*} \mathrm{Sk}_{s-1} \mathrm{~K}\left(G_{s}\right)$ et

$$
\left(\mathrm{T}_{n} \mathrm{G}(S)\right) / \mathrm{F}^{s}=\left(\widehat{\mathrm{MU}}^{*} \operatorname{hom}\left(\mathrm{B} \mathbb{Z} / p^{n}, \mathrm{~K}(S)\right)\right) / \mathrm{F}^{s} \longrightarrow \widehat{\mathrm{MU}}^{*} \mathrm{Sk}_{s-1} \operatorname{hom}\left(\mathrm{B} \mathbb{Z} / p^{n}, \mathrm{~K}(S)\right)
$$

sont des pro-isomorphismes en $s$ par le lemme 3.1.3. En utilisant le pro-isomorphisme $\left(\mathrm{G}\left(G_{s}\right) / \mathrm{F}^{s}\right)_{s} \cong\left(G_{s}\right)_{s}$ et en passant à la limite sur $s$ on en déduit un isomorphisme canonique $G_{\infty} \cong \mathrm{T}_{n} \mathrm{G}(S)$.

Remarque. - Le fait que les objets $\left(\mathrm{Sk}_{s} \operatorname{hom}\left(\mathrm{BZ} / p^{n}, \mathrm{~K}(S)\right)\right)_{s}$ et $\left(\mathrm{Sk}_{s-1} \mathrm{~K}\left(G_{s}\right)\right)_{s}$ sont isomorphes dans ind-h $\widehat{\mathcal{S}}^{0}$ et que les espaces profinis $\mathrm{K}\left(G_{s}\right)$ sont sans $p$-torsion permet de retrouver le fait que l'espace profini hom $\left(\mathrm{B} \mathbb{Z} / p^{n}, \mathrm{~K}(S)\right)$ est sans $p$-torsion, ceci sans utiliser les propriétés magiques du foncteur $\mathrm{T}$ en cohomologie modulo $p$ qui interviennent dans la démonstration de la proposition 6.3. Voici le lemme dont on a besoin :

LEMme 8.8. - Soit $X$ un espace profini tel que la suite de ses squelettes est isomorphe dans ind-h $\widehat{\mathcal{S}}^{0} \grave{a}$ une suite d'espaces profinis sans $p$-torsion; alors $X$ est sans p-torsion.

Démonstration. — Pour chaque entier $v$ le morphisme entre tours

$$
\left(\left(\mathrm{HZ} / p^{v}\right)^{*} \mathrm{Sk}_{s} X\right)_{s} \longrightarrow\left(\mathrm{H}^{*} \mathrm{Sk}_{s} X\right)_{s}
$$

est un pro-épimorphisme. On en déduit par passage à la limite que le morphisme $\left(\mathrm{H} \mathbb{Z} / p^{v}\right)^{*} X \rightarrow \mathrm{H}^{*} X$ est un épimorphisme, d'où le résultat. 
Corollaire 8.9. - Soient $M, N$ deux MU-algèbres instables. On suppose que $N$ est complète pour la filtration squelettale; alors on a une bijection naturelle

$$
\operatorname{Hom}_{\mathcal{K}_{\mathrm{MU}}}\left(\mathrm{T}_{n} M, N\right) \cong \operatorname{Hom}_{\mathcal{K}_{\mathrm{MU}}}\left(M, \mathrm{P}_{n} \widehat{\otimes} N\right) \text {. }
$$

Démonstration. - Supposons d'abord que $M$ est une G-algèbre libre. On a pour tout entier $s$ une bijection naturelle $\operatorname{Hom}_{\mathcal{K}_{\mathrm{MU}}}\left(\mathrm{T}_{n} M, N / \mathrm{F}^{s}\right) \cong \operatorname{Hom}_{\mathcal{K}_{\mathrm{MU}}}\left(M, \mathrm{P}_{n} \widehat{\otimes} N / \mathrm{F}^{s}\right)$ par les résultats de représentabilité qui précèdent et la proposition 8.7. On en déduit la bijection cherchée en passant à la limite sur $s$ et en utilisant l'isomorphisme $\mathrm{P}_{n} \widehat{\otimes} \lim _{s} N / \mathrm{F}^{s} \stackrel{\sim}{\longrightarrow} \lim _{s}\left(\mathrm{P}_{n} \widehat{\otimes} N / \mathrm{F}^{s}\right)$ donné par le point (a) de la proposition 8.3. Le cas $M$ quelconque s'obtient en écrivant $M$ comme le coégalisateur du début de sa G-résolution simpliciale canonique.

Corollaire 8.10. - On a pour toute paire de MU-algèbres instables $M, N$ une bijection naturelle

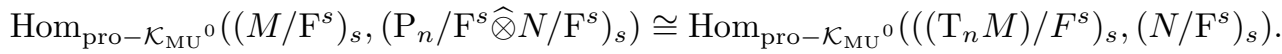

Démonstration. — Le morphisme

$$
\lim _{s}\left(P_{n} \widehat{\otimes} N / \mathrm{F}^{s}\right) \longrightarrow \lim _{s, t}\left(P_{n} / \mathrm{F}^{t} \widehat{\otimes} N / \mathrm{F}^{s}\right) \cong \lim _{s}\left(P_{n} / \mathrm{F}^{s} \widehat{\otimes} N / \mathrm{F}^{s}\right)
$$

est un isomorphisme par la proposition $8.2\left(N / \mathrm{F}^{s}\right.$ est de présentation en degré borné). On en déduit avec le corollaire précédant une bijection naturelle $\lim _{s} \operatorname{Hom}_{\mathcal{K}_{\mathrm{MU}}}\left(\mathrm{T}_{n} M, N / \mathrm{F}^{s}\right) \cong \lim _{s} \operatorname{Hom}_{\mathcal{K}_{\mathrm{MU}}}\left(M, \mathrm{P}_{n} / \mathrm{F}^{s} \widehat{\otimes} N / \mathrm{F}^{s}\right)$. D'autre part pour toute paire de MU-algèbres instables $M, N$ avec $N$ nulle en degré assez grand l'application $\operatorname{colim}_{s} \operatorname{Hom}_{\mathcal{K}_{\mathrm{MU}}}\left(M / \mathrm{F}^{s}, N\right) \rightarrow \operatorname{Hom}_{\mathcal{K}_{\mathrm{MU}}}(M, N)$ est une bijection.

\section{REMARQUES}

- Soit $n_{1}, \ldots, n_{r}$ une suite d'éléments de $\mathbb{N} \cup\{\infty\}$. La proposition 8.3 permet de montrer par récurrence sur $r$ que le morphisme $\mathrm{P}_{n_{1}} \widehat{\otimes} \cdots \widehat{\otimes} \mathrm{P}_{n_{r}} \widehat{\otimes} \widehat{\mathrm{MU}}{ }^{*} X \rightarrow$ $\widehat{\mathrm{MU}}^{*}\left(\mathrm{~B} \mathbb{Z} / p^{n_{1}} \times \cdots \times \mathrm{B} \mathbb{Z} / p^{n_{r}} \times X\right)$ est un isomorphisme pour tout espace profini $X$ dont la MU-cohomologie continue est complète pour la filtration squelettale.

- En utilisant l'isomorphisme $\mathrm{T}_{n_{1}} \cdots \mathrm{T}_{n_{r}} \cong \mathrm{T}_{\mathrm{B}\left(\mathbb{Z} / p^{n_{1}} \times \cdots \times \mathbb{Z} / p^{n_{r}}\right)}$ on obtient par récurrence une formule d'adjonction pour $\mathrm{T}_{\mathrm{B}\left(\mathbb{Z} / p^{n_{1}} \times \cdots \times \mathbb{Z} / p^{n_{r}}\right)}$.

- Soit $\pi$ un groupe de Lie compact commutatif. En choisissant un morphisme $\mathbb{Z} / p^{n_{1}} \times \cdots \times \mathbb{Z} / p^{n_{r}} \rightarrow \pi$ induisant un isomorphisme entre les cohomologies modulo $p$ des classifiants, on obtient une formule d'adjonction pour $\mathrm{T}_{\mathrm{B} \pi}$. 
APPENDICE

\section{A. Monades et algèbres sur une monade}

Les notions et résultats qui suivent sont classiques en théorie des catégories; nous renvoyons à [Bor, Chap.4] pour un exposé plus général. L'exposé qui suit est motivé par notre usage constant des monades et algèbres sur une monades pour d'une part exprimer les structures additives et d'algèbre instable de la MU-cohomologie continue d'un espace profini et d'autre part construire certains foncteurs associés à ces structures (produit tensoriel, foncteurs de division). Son originalité tient à ce que nous nous restreignons aux monades à valeurs dans la catégories des groupes abéliens gradués (plus généralement dans la catégorie des objets en groupe abélien d'une catégorie sous certaines conditions sur cette catégorie). Les coégalisateurs réflexifs d'algèbres sur une telle monade sont scindés dans la catégorie des ensembles gradués, ce qui entraîne des propriétés d'exactitude très fortes des foncteurs définis sur la catégorie des algèbres sur la monade.

La première section (A.1) traite des coégalisateurs de 1-complexes (ou coégalisateurs réflexifs) d'objets en groupe abélien (proposition A.1.2) pour ensuite montrer l'existence des colimites de diagrammes d'algèbres sur une monade donnée $T$ (proposition A.1.5) et donner un critère d'exactitude à droite d'un foncteur défini sur les $T$-algèbres sous certaines hypothèses sur $T$ (proposition A.1.6). Elle se termine par la notion de sous- $T$-algèbre et de $T$-algèbre quotient lorsque $T$ est une monade sur la catégorie des ensembles gradués.

La section A.2 considère la monade associée à une paire de foncteurs adjoints et montre comment on étend par exactitude à droite un foncteur défini sur les objets libres en un foncteur défini sur toutes les algèbres associées à la monade (proposition A.2.2). On en déduit une variante du théorème de Beck (proposition A.2.4).

Dans la section A.3 on applique ce qui précède pour caractériser les monades dont la catégorie d'algèbres associée est abélienne (proposition A.3.2). 
La dernière section (A.4) traite des résolutions : notion de complexe augmenté acyclique relativement à une classe d'objets en cogroupe abélien, version simpliciale, résolutions dans la catégorie des algèbres sur une monade. Nous renvoyons à [BB] pour des compléments sur ce dernier point.

A.1. Diagrammes coégalisateurs et monades. - Soit $\mathcal{C}$ une catégorie. Nous appelons 1-complexe de $\mathcal{C}$ et notons $C_{1} \leftrightarrows C_{0}$ un couple de morphismes $\mathrm{d}_{0}, \mathrm{~d}_{1}: C_{1} \rightarrow$ $C_{0}$ entre deux objets de $\mathcal{C}$ muni d'une section commune so $: C_{0} \rightarrow C_{1}$. Dualement nous appelons 1-cocomplexe de $\mathcal{C}$ un couple de morphismes $C^{0} \rightarrow C^{1}$ muni d'un rétract commun.

Nous dirons qu'un diagramme $C_{1} \leftrightarrows C_{0} \rightarrow C$ est coégalisateur si le morphisme $C_{0} \rightarrow C$ fait de $C$ le coégalisateur des deux morphismes $\mathrm{d}_{0}$ et $\mathrm{d}_{1}: C_{1} \rightarrow C_{0}$ (Nous dirons alors aussi que $C$ est le coégalisateur du 1-complexe $\left.C_{1} \leftrightarrows C_{0}\right)$. Idem pour les diagrammes égalisateurs. Observons que si $C_{0}$ et $C_{1}$ sont deux objets de $\mathcal{C}$ admettant une somme $C_{1} \sqcup C_{0}$ dans $\mathcal{C}$, le coégalisateur d'un couple de morphismes $C_{1} \rightrightarrows C_{0}$ coïncide avec le coégalisateur du 1-complexe $C_{1} \sqcup C_{0} \leftrightarrows C_{0}$ obtenu en prenant l'identité sur $C_{0}$.

Soient $f$ et $g$ deux morphismes entre 1-complexes $C_{1} \leftrightarrows C_{0}$ et $D_{1} \leftrightarrows D_{0} \quad(f=$ $\left(f_{0}: C_{0} \rightarrow D_{0}, f_{1}: C_{1} \rightarrow D_{1}\right)$, etc.). Une homotopie en degré 0 de $f$ vers $g$ est un morphisme $h: C_{0} \rightarrow D_{1}$ tel que $\mathrm{d}_{0} h=f_{0}$ et $\mathrm{d}_{1} h=g_{0}$. Nous dirons qu'un morphisme $f: \triangle \rightarrow \triangle^{\prime}$ entre 1-complexes est une équivalence d'homotopie en degré 0 s'il existe un morphisme $g: \triangle^{\prime} \rightarrow \triangle$ et des homotopies en degré 0 de $g f$ vers l'identité de $\triangle$ et de $f g$ vers l'identité de $\triangle^{\prime}$.

Exemple. - La donnée d'un morphisme d'un 1-complexe $C_{1} \leftrightarrows C_{0}$ dans le 1complexe constant $C \stackrel{\leftrightarrows}{\leftrightarrows}$ associé à un objet $C$ équivaut à la donnée d'un morphisme $g: C_{0} \rightarrow C$ telle que les composées $g \mathrm{~d}_{0}$ et $g \mathrm{~d}_{1}$ soient égales. Un tel morphisme est une équivalence d'homotopie en degré 0 si et seulement s'il existe des morphismes $s: C \rightarrow C_{0}$ et $s^{\prime}: C_{0} \rightarrow C_{1}$ tels qu'on ait les identités

$$
g s=\operatorname{Id}_{C}, \mathrm{~d}_{1} s^{\prime}=\operatorname{Id}_{C_{0}}, \mathrm{~d}_{0} s^{\prime}=s g .
$$

LEMME A.1.1

(a) Soient $\triangle, \triangle^{\prime}$ deux 1-complexes de $\mathcal{C}$ admettant un coégalisateur et $f, g$ deux morphismes $\triangle \rightarrow \triangle^{\prime}$. Si $f$ est homotope en degré 0 à $g$ alors $f$ et $g$ induisent les mêmes morphismes entre les coégalisateurs.

(b) Soient $C$ un objet de $\mathcal{C}$ et $f$ un morphisme d'un 1-complexe $C_{1} \leftrightarrows C_{0}$ dans le diagramme constant $C \leftrightarrows C$. Si f est une équivalence d'homotopie en degré 0 alors le diagramme $C_{1} \underset{\leftrightarrows}{\leftrightarrows} C_{0} \rightarrow C$ est coégalisateur.

Démonstration. - Le point (a) est facile. Pour le point (b) choisissons des morphismes $s, s^{\prime}$ vérifiant les identités $f_{0} s=\operatorname{Id}_{C}, \mathrm{~d}_{1} s^{\prime}=\operatorname{Id}_{C_{0}}, \mathrm{~d}_{0} s^{\prime}=s f_{0}$. Soit $g: C_{0} \rightarrow D$ un morphisme dans $\mathcal{C}$ égalisant $\mathrm{d}_{0}$ et $\mathrm{d}_{1}$; alors $g$ est égal à la composée $g s f_{0}$ donc 
se factorise par $C_{0} \rightarrow C$. Cette factorisation est unique car $C_{0} \rightarrow C$ admet une section.

Nous dirons qu'un diagramme $C_{1} \leftrightarrows C_{0} \rightarrow C$ est un diagramme coégalisateur scindé (ou que $C$ est un coégalisateur scindé du 1-complexe $C_{1} \leftrightarrows C_{0}$ ) si le morphisme $C_{0} \rightarrow C$ induit une équivalence d'homotopie en degré 0 de $C_{1} \leftrightarrows C_{0}$ dans le 1-complexe constant $C \leftrightarrows C$. La propriété d'être scindé est préservée par tout foncteur.

On a bien sûr le même formalisme et les mêmes énoncés pour les 1-cocomplexes de $\mathcal{C}$.

Si $\mathcal{C}$ possède tous les produits finis (en particulier un objet terminal), on note $\mathcal{C}_{\mathrm{ab}}$ la catégorie des objets en groupe abélien de $\mathcal{C}$, c'est-à-dire $\operatorname{des}$ objets $C \in \mathcal{C}$ munis d'un morphisme $C \times C \rightarrow C$ faisant de $\operatorname{Hom}_{\mathcal{C}}\left(C^{\prime}, C\right)$ un groupe abélien naturel en $C^{\prime} \in \mathcal{C}$. Tout diagramme de $\mathcal{C}_{\text {ab }}$ qui admet une limite dans $\mathcal{C}$ admet une limite dans $\mathcal{C}_{\text {ab }}$ et l'oubli $\mathcal{C}_{\mathrm{ab}} \rightarrow \mathcal{C}$ commute aux limites. En particulier l'objet terminal de $\mathcal{C}$ est l'objet nul de $\mathcal{C}_{\mathrm{ab}}$. Les sommes finies coïncident avec les produits finis dans $\mathcal{C}_{\mathrm{ab}}$.

Soit alors $M_{1} \leftrightarrows M_{0}$ un 1-complexe de $\mathcal{C}_{\text {ab }}$ et supposons l'existence des noyaux. La composée $\mathrm{s}_{1} \mathrm{~d}_{1}$ est un projecteur de $M_{1}$; notons $M_{1}^{\prime}$ son noyau et d la restriction de $\mathrm{d}_{0}$ à $M_{1}^{\prime}$. On dispose d'un isomorphisme canonique dans $\mathcal{C}_{\text {ab }}$ du 1-complexe $M_{1} \rightleftarrows M_{0}$ dans le 1-complexe $\left(\mathrm{d}, \operatorname{Id}_{M_{0}}\right),\left(0, \operatorname{Id}_{M_{0}}\right),\left(0, \operatorname{Id}_{M_{0}}\right): M_{1}^{\prime} \oplus M_{0} \leftrightarrows M_{0}$. La catégorie des 1complexes de $\mathcal{C}_{\mathrm{ab}}$ est donc équivalente à celle des morphismes de $\mathcal{C}_{\mathrm{ab}}$. Le coégalisateur dans $\mathcal{C}_{\text {ab }}$ de $M_{1} \leftrightarrows M_{0}$ s'identifie via cette équivalence au conoyau de d. Pour toute paire de morphismes $f, g$ entre 1-complexes de $\mathcal{C}_{\mathrm{ab}}$, une homotopie en degré 0 dans $\mathcal{C}_{\mathrm{ab}}$, respectivement dans $\mathcal{C}$, de $f$ vers $g$ s'identifie via cette équivalence à un relèvement dans $\mathcal{C}_{\text {ab }}$, respectivement dans $\mathcal{C}$, de $f-g$ par rapport à d. En particulier la relation d'homotopie en degré 0 dans $\mathcal{C}_{\text {ab }}$ ou dans $\mathcal{C}$ entre morphismes de 1 -complexes de $\mathcal{C}_{\text {ab }}$ est une relation d'équivalence.

Proposition A.1.2. - Soit $\mathcal{C}$ une catégorie possédant toutes les limites et colimites finies. Les conditions suivantes sont équivalentes :

(i) Pour tout objet $C \in \mathcal{C}$ le foncteur $\operatorname{Hom}_{\mathcal{C}}(C,-)$ transforme le coégalisateur dans $\mathcal{C}$ d'un 1-complexe de $\mathcal{C}_{\mathrm{ab}}$ en le coégalisateur du 1-complexe image.

(ii) Le coégalisateur dans $\mathcal{C}$ d'un 1-complexe de $\mathcal{C}_{\mathrm{ab}}$ est un coégalisateur scindé.

Si elles sont vérifiées, la catégorie $\mathcal{C}_{\mathrm{ab}}$ est abélienne et l'oubli $\mathcal{C}_{\mathrm{ab}} \rightarrow \mathcal{C}$ commute aux coégalisateurs de 1-complexes.

Démonstration. — La condition (ii) implique (i).

Supposons la condition (i) vérifiée. Soient $M_{1} \leftrightarrows M_{0}$ un 1-complexe de $\mathcal{C}_{\text {ab }}$ et $M$ son coégalisateur dans $\mathcal{C}$. Alors pour tout objet $C \operatorname{de} \mathcal{C}$ l'ensemble $\operatorname{Hom}_{\mathcal{C}}(C, M)$ hérite de $\operatorname{Hom}_{\mathcal{C}}\left(C, M_{0}\right)$ d'une structure de groupe abélien naturelle en $C$. Autrement dit $M$ hérite de $M_{0}$ d'une structure d'objet en groupe abélien qui en fait le coégalisateur dans $\mathcal{C}_{\mathrm{ab}}$ du 1-complexe donné. Comme le conoyau d'un morphisme s'identifie au 
coégalisateur du 1-complexe associé, on en déduit que les conoyaux existent dans $\mathcal{C}_{\mathrm{ab}}$ et que le foncteur $\operatorname{Hom}_{\mathcal{C}}(C,-)$ (additif) commute aux conoyaux donc est exact.

Soit $M \rightarrow N$ un morphisme dans $\mathcal{C}_{\text {ab }}$. On dispose d'un morphisme

$$
\operatorname{Coker}(\operatorname{Ker}(M \longrightarrow N) \longrightarrow M) \longrightarrow \operatorname{Ker}(N \longrightarrow \operatorname{Coker}(M \longrightarrow N))
$$

dont l'image par $\operatorname{Hom}_{\mathcal{C}}(C,-)$ est un isomorphisme quelque soit $C \in \mathcal{C}$ donc qui est iso dans $\mathcal{C}$ donc iso dans $\mathcal{C}_{\text {ab }}$. Autrement dit la catégorie $\mathcal{C}_{\text {ab }}$ est abélienne.

Il reste à montrer que la condition (ii) est satisfaite. Il suffit pour cela de montrer que si $f: M \rightarrow N$ et $g: N \rightarrow P$ sont des morphismes dans $\mathcal{C}_{\text {ab }}$ tels que la suite $M \rightarrow N \rightarrow P \rightarrow 0$ est exacte alors il existe des morphismes $s: P \rightarrow N$ et $s^{\prime}:$ $N \rightarrow M$ dans $\mathcal{C}$ tels que $g s=\operatorname{Id}_{P}$ et $s g-f s^{\prime}=\operatorname{Id}_{N}$. L'existence de $s$ vient de ce que le morphisme de groupes abéliens $\operatorname{Hom}_{\mathcal{C}}(P, N) \rightarrow \operatorname{Hom}_{\mathcal{C}}(P, P)$ est surjectif car de conoyau nul. L'existence de $s^{\prime}$ vient de ce que le morphisme $g\left(s g-\operatorname{Id}_{N}\right)=g s g-g$ est nul et de ce que la suite

$$
\operatorname{Hom}_{\mathcal{C}}(N, M) \longrightarrow \operatorname{Hom}_{\mathcal{C}}(N, N) \longrightarrow \operatorname{Hom}_{\mathcal{C}}(N, P) \longrightarrow 0
$$

est exacte.

Exemple. - Prenons pour $\mathcal{C}$ la catégorie des ensembles; alors $\mathcal{C}_{\text {ab }}$ est la catégorie des groupes abéliens et la condition (i) de la proposition est vérifiée. L'exemple le plus important pour notre travail est en fait la catégorie $\mathcal{E}$ ns-gr des ensembles gradués (Ens-gr ab est la catégorie des groupes abéliens gradués). Nous rencontrerons aussi la catégorie produit $\mathcal{E}$ ns-gr $\times \mathcal{E}$ ns-gr.

L'importance pour notre propos des diagrammes coégalisateurs scindés apparaît par les propositions A.1.4 et A.1.5 ci-dessous. Nous commençons par définir :

Une monade sur une catégorie $\mathcal{C}$ est un endofoncteur $T$ de $\mathcal{C}$ muni de transformations naturelles $\eta: \operatorname{Id} \rightarrow T$ et $\mu: T^{2} \rightarrow T$ telles que pour tout objet $C$ de $\mathcal{C}$

- les composées $\mu(C) \circ T(\mu(C))$ et $\mu(C) \circ \mu(T(C)): T^{3}(C) \rightarrow T(C)$ sont égales;

- les composées $\mu(C) \circ T(\eta(C))$ et $\mu(C) \circ \eta(T(C)): T(C) \rightarrow T(C)$ sont l'identité.

Soit $T$ une monade sur $\mathcal{C}$. Une $T$-algèbre est un objet $M$ muni d'un morphisme $\alpha: T(M) \rightarrow M$ tel que

- les composées $\alpha \circ \mu(M)$ et $\alpha \circ T(\alpha): T^{2}(M) \rightarrow M$ sont égales;

- la composée $\alpha \circ \eta(M): M \rightarrow M$ est l'identité.

Si $M$ et $M^{\prime}$ sont deux $T$-algèbres, un morphisme de $T$-algèbres de $M$ dans $M^{\prime}$ est un morphisme $M \rightarrow M^{\prime}$ dans $\mathcal{C}$ tel que le diagramme induit

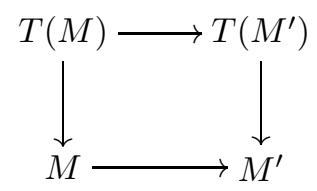


commute. En particulier un morphisme de $T$-algèbres est un isomorphisme si et seulement si le morphisme de $\mathcal{C}$ sous-jacent est un isomorphisme. Les $T$-algèbres et leurs morphismes forment une catégorie qu'on note $\mathcal{C}(T)$. On dispose d'un foncteur oubli évident $\mathcal{C}(T) \rightarrow \mathcal{C}$.

Soit $C$ un objet de $\mathcal{C}$; le morphisme $\mu(C): T^{2}(C) \rightarrow T(C)$ fait de $T(C)$ une $T$-algèbre naturelle en $C$ et le morphisme $\eta(C): C \rightarrow T(C)$ induit pour toute $T$ algèbre $N$ une bijection $\operatorname{Hom}_{\mathcal{C}(T)}(T(C), N) \rightarrow \operatorname{Hom}_{\mathcal{C}}(C, N): T(C)$ est la $T$-algèbre libre sur $C$. Plus généralement on appelle $T$-algèbre libre une $T$-algèbre isomorphe à $T(C)$ pour un certain objet $C \in \mathcal{C}$.

Soit $M$ une $T$-algèbre; le diagramme

$$
T^{2}(M) \rightleftarrows T(M) \longrightarrow M
$$

de $\mathcal{C}$ formé des morphismes $d_{0}=\mu(M), d_{1}=T(\alpha), s_{0}=T(\eta(M))$ et $\alpha$ est un diagramme coégalisateur canoniquement scindé par les morphismes $\eta(M)$ et $\eta(T(M))$. On vérifie d'autre part que le morphisme $\alpha: T(M) \rightarrow M$ est un morphisme de $T$ algèbres de sorte que le diagramme $T^{2}(M) \leftrightarrows T(M) \rightarrow M$ est un diagramme dans $\mathcal{C}(T)$ naturel en $M \in \mathcal{C}(T)$. Il est coégalisateur dans $\mathcal{C}(T)$ par le lemme ci-dessous mais pas coégalisateur scindé en général.

Lemme A.1.3. - Soit $M_{1} \leftrightarrows M_{0}$ un 1-complexe de T-algèbres admettant un coégalisateur $M$ dans $\mathcal{C}$. On suppose que les images par $T$ et $T^{2}$ du diagramme $M_{1} \leftrightarrows M_{0} \rightarrow$ $M$ sont encore des diagrammes coégalisateurs dans $\mathcal{C}$; alors $M$ hérite d'une structure naturelle de T-algèbre faisant de lui le coégalisateur du 1-complexe $M_{1} \leftrightarrows M_{0}$ dans $\mathcal{C}(T)$.

Démonstration. — Si les diagrammes

$$
T\left(M_{1}\right) \rightleftarrows T\left(M_{0}\right) \longrightarrow T(M) \quad \text { et } \quad T^{2}\left(M_{1}\right) \rightleftarrows T^{2}\left(M_{0}\right) \longrightarrow T^{2}(M)
$$

sont des diagrammes coégalisateurs dans $\mathcal{C}$, les morphismes de structure $T\left(M_{1}\right) \rightarrow M_{1}$ et $T\left(M_{0}\right) \rightarrow M_{0}$ induisent un morphisme $T(M) \rightarrow M$ qui fait de $M$ une $T$-algèbre. Comme le morphisme $T\left(M_{0}\right) \rightarrow T(M)$ est épi, tout morphisme de $T$-algèbres de $M_{0}$ dans une $T$-algèbre $N$ qui se factorise dans $\mathcal{C}$ par $M_{0} \rightarrow M$ se factorise dans la catégorie des $T$-algèbres.

On suppose désormais que $\mathcal{C}$ est cocomplète, c'est à dire possède toutes les colimites indexées par une catégorie petite. On note $\left(\mathcal{Q}_{0}\right)$ et $\left(\mathcal{Q}_{1}\right)$ les conditions suivantes :

$\left(\mathcal{Q}_{0}\right)$ Le coégalisateur dans $\mathcal{C}$ d'un 1-complexe de $T$-algèbres est un coégalisateur scindé.

$\left(\mathcal{Q}_{1}\right)$ L'image par $T$ du coégalisateur dans $\mathcal{C}$ d'un 1-complexe de $T$-algèbres est le coégalisateur dans $\mathcal{C}$ du 1-complexe image.

La condition $\left(\mathcal{Q}_{0}\right)$ implique $\left(\mathcal{Q}_{1}\right)$. 
Proposition A.1.4. - Soit $\mathcal{C}$ une catégorie possédant toutes les limites et colimites finies et vérifiant la condition (i) de la proposition A.1.2. Soit $T$ une monade sur $\mathcal{C}$ telle que le foncteur $T$ et la transformation naturelle $T \circ T \rightarrow T$ sont à valeurs dans $\mathcal{C}_{\text {ab }}$; alors $T$ vérifie $\left(\mathcal{Q}_{0}\right)$.

Démonstration. - Soit $M$ une $T$-algèbre; alors $M$ est le coégalisateur scindé dans $\mathcal{C}$ du 1-complexe d'objets en groupes abéliens $T^{2}(M) \leftrightarrows T(M)$ donc hérite d'une structure d'objet en groupe abélien. On en déduit grâce à la proposition A.1.2 que le coégalisateur dans $\mathcal{C}$ d'un 1-complexe de $T$-algèbres est un coégalisateur scindé.

Proposition A.1.5. - Soient $\mathcal{C}$ une catégorie cocomplète et $T$ une monade sur $\mathcal{C}$ vérifiant $\left(\mathcal{Q}_{1}\right)$; alors $\mathcal{C}(T)$ est cocomplète et le foncteur oubli $\mathcal{C}(T) \rightarrow \mathcal{C}$ commute aux coégalisateurs de 1-complexes.

Démonstration. - On observe d'abord que les coégalisateurs de 1-complexes existent dans $\mathcal{C}(T)$ et que l'oubli $\mathcal{C}(T) \rightarrow \mathcal{C}$ commute aux coégalisateurs de 1-complexes par le lemme A.1.3.

Soit $\left(M_{\alpha}\right)$ un diagramme de $T$-algèbres. Chaque $M_{\alpha}$ est le coégalisateur dans $\mathcal{C}(T)$ du 1-complexe $T^{2}\left(M_{\alpha}\right) \leftrightarrows T\left(M_{\alpha}\right)$. Par adjonction, la colimite dans $\mathcal{C}(T)$ du diagramme image par $T$ de $\left(M_{\alpha}\right)$ est l'image par $T$ de la colimite dans $\mathcal{C}$ des $M_{\alpha}$. On obtient donc un 1-complexe $\operatorname{colim}_{\alpha} T^{2}\left(M_{\alpha}\right) \rightrightarrows \operatorname{colim}_{\alpha} T\left(M_{\alpha}\right)$ de $\mathcal{C}(T)$. Son coégalisateur dans $\mathcal{C}(T)$ est la colimite dans $\mathcal{C}(T)$ du diagramme $\left(M_{\alpha}\right)$ par commutation des colimites entre elles.

Notons que le foncteur oubli $\mathcal{C}(T) \rightarrow \mathcal{C}$ ne commute pas aux colimites en général. A l'opposé le foncteur $\widetilde{T}: \mathcal{C} \rightarrow \mathcal{C}(T)$ qui associe à un objet $C$ l'objet $T(C)$ muni de sa structure naturelle de $T$-algèbre est adjoint à gauche de l'oubli $\mathcal{C}(T) \rightarrow \mathcal{C}$ donc commute aux colimites.

Proposition A.1.6. - Soient $\mathcal{C}$ et $\mathcal{D}$ deux catégories cocomplètes, $T$ une monade sur $\mathcal{C}$ vérifiant la condition $\left(\mathcal{Q}_{1}\right)$ et $F$ un foncteur $\mathcal{C}(T) \rightarrow \mathcal{D}$. On suppose que pour toute $T$-algèbre $M$ l'image par $F$ du diagramme $T^{2}(M) \leftrightarrows T(M) \rightarrow M$ est coégalisateur dans $\mathcal{D}$; alors :

(a) F commute aux coégalisateurs de 1-complexes si et seulement si la composée $F \widetilde{T}$ transforme le coégalisateur dans $\mathcal{C}$ d'un 1-complexe de $T$-algèbres en le coégalisateur dans $\mathcal{D}$ du 1-complexe image.

(b) Supposons la condition du (a) vérifiée; alors $F$ commute aux colimites finies si et seulement si F $\widetilde{T}$ transforme la somme de deux objets en la somme des images; $F$ commute aux colimites indexées par une catégorie petite si F $\widetilde{T}$ commute de plus aux colimites filtrantes.

Démonstration. - Pour le point (a), on observe d'abord que l'oubli $\mathcal{C}(T) \rightarrow \mathcal{C}$ commute aux coégalisateurs de 1-complexes par la proposition A.1.5. Soient $M_{1} \leftrightarrows M_{0}$ un 1-complexe de $\mathcal{C}(T)$ et $M$ son coégalisateur. Les diagrammes $M_{1} \rightleftarrows M_{0} \rightarrow M$ et 
$T\left(M_{1} \leftrightarrows M_{0} \rightarrow M\right)$ sont coégalisateurs dans $\mathcal{C}$, donc également leurs images par $F \circ \widetilde{T}$ dans $\mathcal{D}$ par hypothèse. Or $F(M)$ est le coégalisateur dans $\mathcal{D}$ de l'image par $F$ du 1-complexe $T^{2}(M) \leftrightarrows T(M)$ de $\mathcal{C}(T)$, donc est le coégalisateur de l'image par $F \mathrm{du}$ 1-complexe $M_{1} \leftrightarrows M_{0}$ par commutation des colimites entre elles.

Le point (b) s'obtient en exprimant une colimite finie (respectivement une colimite indexée par une catégorie petite) à l'aide de sommes finies (respectivement de sommes finies et de colimites filtrantes) et d'un coégalisateur de 1-complexe, en utilisant le fait que le foncteur $T: \mathcal{C} \rightarrow \mathcal{C}(T)$ commute par adjonction aux colimites, en exprimant une colimite de $T$-algèbres comme le coégalisateur d'un 1-complexe de $T$-algèbres libres ( $c f$. la démonstration de la proposition A.1.5) et en utilisant la commutation des colimites entre elles.

La condition du (a) est automatiquement vérifiée si la monade $T$ vérifie $\left(\mathcal{Q}_{0}\right)$.

Dualement si $\mathcal{C}$ est complète alors tout diagramme $\left(M_{\alpha}\right)$ de $T$-algèbres indexé par une catégorie petite admet une limite et l'oubli $\mathrm{C}(T) \rightarrow \mathcal{C}$ commute aux limites. L'objet de $\mathcal{C}$ sous-jacent à la limite des $M_{\alpha}$ est la limite dans $\mathcal{C}$ du diagramme $\left(M_{\alpha}\right)$ et le morphisme $T\left(\lim _{\alpha} M_{\alpha}\right) \rightarrow \lim _{\alpha} M_{\alpha}$ est donné par les composées $T\left(\lim _{\alpha} M_{\alpha}\right) \rightarrow$ $T\left(M_{\beta}\right) \rightarrow M_{\beta}$ induites par les projections $\lim _{\alpha} M_{\alpha} \rightarrow M_{\beta}$ et la structure de $T$-algèbre $\operatorname{des} M_{\beta}$.

Puisque l'oubli $\mathrm{C}(T) \rightarrow \mathcal{C}$ est fidèle et admet un adjoint à gauche, un morphisme de $\mathcal{C}(T)$ est un monomorphisme si et seulement si le morphisme de $\mathcal{C}$ sous-jacent est un monomorphisme.

Cas particulier : Sous-objets et objets quotient des algèbres associées à une monade sur $\mathcal{E}$ ns-gr. - Soient $T$ une monade sur la catégorie $\mathcal{E}$ ns-gr des ensembles gradués et $M$ une $T$-algèbre. Une sous- $T$-algèbre de $M$ est un sous-ensemble gradué $M^{\prime}$ muni d'une structure de $T$-algèbre telle que l'inclusion $M^{\prime} \rightarrow M$ soit un morphisme de $T$ algèbres. De même une $T$-algèbre quotient de $M$ est un ensemble gradué quotient muni d'une structure de $T$-algèbre compatible. Les structures compatibles de $T$-algèbre sur un sous-ensemble gradué ou un ensemble gradué quotient, lorsqu'elles existent, sont uniques. Si $M^{\prime}$ est une sous- $T$-algèbre de $M$ (respectivement une $T$-algèbre quotient), le morphisme $M^{\prime} \rightarrow M$ est un monomorphisme de $\mathcal{E}$ ns-gr $(T)$ (respectivement $M \rightarrow$ $M^{\prime}$ est un épimorphisme de $\mathcal{E}$ ns-gr $\left.(T)\right)$.

Soit $M \rightarrow M^{\prime}$ un morphisme de $T$-algèbres. On lui associe le 1-complexe de $T$ algèbres $M \times_{M^{\prime}} M \leftrightarrows M$. Notons $Q$ le coégalisateur dans $\mathcal{E}$ ns-gr de ce 1-complexe; c'est donc un ensemble gradué quotient de $M$. Comme le diagramme

$$
M \times{ }_{M^{\prime}} M \rightleftarrows M \longrightarrow Q
$$

est un diagramme coégalisateur scindé dans $\mathcal{E}$ ns-gr, $Q$ hérite de $M$ d'une structure de $T$-algèbre faisant de lui le coégalisateur de $M \times_{M^{\prime}} M \leftrightarrows M$ dans $\mathcal{E}$ ns-gr $(T)$ (lemme A.1.3). Comme le morphisme $M \rightarrow M^{\prime}$ égalise les deux morphismes $M \times{ }_{M^{\prime}} M \rightarrow M$, il se factorise par la projection $M \rightarrow Q$. Comme l'application $Q \rightarrow M^{\prime}$ est injective 
en chaque degré, on en déduit une structure de $T$-algèbre sur l'image de $M$ dans $M^{\prime}$ isomorphe à celle de $Q$. Autrement dit le morphisme de $T$-algèbres $M \rightarrow M^{\prime}$ s'écrit de façon unique comme la composée de la projection de $M$ sur une $T$-algèbre quotient avec un isomorphisme de ce quotient dans une sous-T-algèbre de $M^{\prime}$.

Exemple. - Soient $M$ une $T$-algèbre et $S$ un sous-ensemble gradué de $M$. L'image du morphisme $T(S) \rightarrow M$ (adjoint de l'inclusion $S \subset M$ ) est la plus petite sous- $T$ algèbre de $M$ contenant $S$.

Observons au passage que si $M \rightarrow M^{\prime}$ est un morphisme de $T$-algèbres et $Q$ une $T$-algèbre quotient de $M$, alors $M \rightarrow M^{\prime}$ se factorise par la projection $M \rightarrow Q$ dans $\mathcal{E}$ ns-gr $(T)$ si et seulement si il se factorise par $M \rightarrow Q$ dans $\mathcal{E}$ ns-gr, ceci parce que chacune des conditions est équivalente à $M \rightarrow M^{\prime}$ égalise les deux morphismes $M \times{ }_{Q} M \rightarrow M$.

A.2. Monades et adjonctions. - Soient $\mathcal{C}$ et $\mathcal{D}$ deux catégories et soit $O: \mathcal{D} \rightarrow \mathcal{C}$ un foncteur admettant un adjoint à gauche $L$. L'unité $\eta: I d \rightarrow O L$ et la transformation naturelle $O \epsilon L: O L O L \rightarrow O L$ induite par la counité $\epsilon: L O \rightarrow$ Id munissent la composée $T=O L$ d'une structure de monade sur $\mathcal{C}$. Dualement la composée $L O$ a une structure naturelle de comonade sur $\mathcal{D}$. (On donne un sens précis à cette dualité en considérant la catégorie opposée à la catégorie des catégories.)

Pour toute $O L$-algèbre $M$ le 1-complexe $(O L)^{2}(M) \leftrightarrows(O L)(M)$ est l'image par $O$ du 1-complexe $L O L(M) \rightrightarrows L(M)$ de $\mathcal{D}$ formé des morphismes $\epsilon(L(M)), L(\alpha)$ et $L(\eta(M))$. Tout morphisme $M \rightarrow M^{\prime}$ de $O L$-algèbres induit un morphisme entre les 1-complexes $L O L(M) \rightrightarrows L(M)$ et $L O L\left(M^{\prime}\right) \rightleftarrows L\left(M^{\prime}\right)$ de $\mathcal{D}$.

Soit $M$ un objet de $\mathcal{D}$. Le morphisme $O(\epsilon(M)): O L O(M) \rightarrow O(M)$ munit $O(M)$ d'une structure de $O L$-algèbre donc définit un relèvement $\mathcal{D} \rightarrow \mathcal{C}(O L)$ du foncteur $O: \mathcal{D} \rightarrow \mathcal{C}$, que nous noterons $\widetilde{O}$ si besoin. Le diagramme associé

$$
(O L)^{2} O(M) \rightleftarrows(O L) O(M) \longrightarrow O(M),
$$

qui, rappelons le, est canoniquement un diagramme coégalisateur scindé dans $\mathcal{C}$ donc coégalisateur dans $\mathcal{C}(O L)$, est l'image par $O$ du diagramme

$$
(L O)^{2}(M) \rightleftarrows L O(M) \longrightarrow M
$$

issu de l'adjonction entre $L$ et $O$.

On note

- $L(\mathcal{C})$ la sous-catégorie pleine de $\mathcal{D}$ formée des objets $L(C), C$ décrivant $\mathcal{C}$,

- $\mathcal{L}_{\mathcal{D}}$ la sous-catégorie pleine de $\mathcal{D}$ formée des objets isomorphes à un objet de $L(\mathcal{C})$ et

- $\mathcal{L}_{\mathcal{C}(O L)}$ la sous-catégorie pleine de $\mathcal{C}(O L)$ formée des $O L$-algèbres libres.

(L'inclusion de $L(\mathcal{C})$ dans $\mathcal{L}_{\mathcal{D}}$ est une équivalence de catégories.) 


\section{LEMME A.2.1}

(a) Le foncteur $\widetilde{O}: \mathcal{D} \rightarrow \mathcal{C}(O L)$ induit une équivalence de catégories $\mathcal{L}_{\mathcal{D}} \rightarrow \mathcal{L}_{\mathcal{C}(O L)}$.

(b) Soit $M$ un objet de $\mathcal{L}_{\mathcal{D}}$; alors le diagramme $(L O)^{2}(M) \leftrightarrows L O(M) \rightarrow M$ est un diagramme coégalisateur scindé dans $\mathcal{L}_{\mathcal{D}}$.

Démonstration. - Pour tout objet $C$ de $\mathcal{C}$, l'image par $\widetilde{O}$ de $L(C)$ est la $O L$-algèbre libre sur $C$. Soient $C$ et $C^{\prime}$ deux objets de $\mathcal{C}$. On a par adjonction des bijections

$$
\operatorname{Hom}_{\mathcal{D}}\left(L(C), L\left(C^{\prime}\right)\right) \simeq \operatorname{Hom}_{\mathcal{C}}\left(C, O L\left(C^{\prime}\right)\right) \simeq \operatorname{Hom}_{\mathcal{C}(O L)}\left(O L(C), O L\left(C^{\prime}\right)\right)
$$

d'où le point (a).

Pour le point (b) il suffit de choisir un isomorphisme $M \simeq L(C)$ et d'observer que le diagramme $(L O)^{2}(L(C)) \rightleftarrows L O(L(C)) \rightarrow L(C)$ est canoniquement scindé dans $\mathcal{D}$ par les morphismes $L(\eta(C))$ et $L\left(\eta_{O L}(C)\right)$.

EXEMPLE. - Soit $T$ une monade sur $\mathcal{C}$; prenons pour $\mathcal{D}$ la catégorie $\mathcal{C}(T)$ et pour $O$ le foncteur oubli $\mathcal{C}(T) \rightarrow \mathcal{C}$. Le foncteur $\widetilde{T}$ est adjoint à gauche de $O$ et le foncteur $\widetilde{O}: \mathcal{C}(T) \rightarrow \mathcal{C}(O \widetilde{T})=\mathcal{C}(T)$ est une équivalence de catégories (c'est l'identité). La proposition A.2.4 donnera une réciproque.

Proposition A.2.2. - Soient $\mathcal{D}^{\prime}$ une catégorie cocomplète et $F$ un foncteur $L(\mathcal{C}) \rightarrow$ $\mathcal{D}^{\prime}$; alors il existe un foncteur $\widetilde{F}: \mathcal{C}(O L) \rightarrow \mathcal{D}^{\prime}$ unique à isomorphisme près vérifiant

(1) La composée $L(\mathcal{C}) \rightarrow \mathcal{C}(O L) \rightarrow \mathcal{D}^{\prime}$ est isomorphe à $F$.

(2) Pour toute $O L$-algèbre $M$ l'image par $\widetilde{F}$ du diagramme

$$
(O L)^{2}(M) \rightleftarrows O L(M) \longrightarrow M
$$

est coégalisateur.

Démonstration. - Si $\widetilde{F}$ existe alors pour tout $C \in \mathcal{C}, \widetilde{F}(O L(C))$ est isomorphe à $F(L(C))$ naturellement en $L(C)$ par la propriété $(1)$ de sorte que pour toute $O L$ algèbre $M, \widetilde{F}(M)$ est naturellement isomorphe au coégalisateur dans $\mathcal{D}^{\prime}$ de l'image par $F$ du 1-complexe $L O L(M) \leftrightarrows L(M)$, d'où l'unicité.

Pour l'existence on définit pour $M \in \mathcal{C}(O L) \widetilde{F}(M)$ comme ce coégalisateur. Pour tout objet $C$ de $\mathcal{C}$ le diagramme

$$
(L O)^{2}(L(C)) \rightleftarrows L O(L(C)) \longrightarrow L(C)
$$

est (canoniquement) un diagramme coégalisateur scindé dans $L(\mathcal{C})$, donc également son image par $F$ dans $\mathcal{D}^{\prime}$. On en déduit un isomorphisme canonique $\widetilde{F}(O L(C)) \rightarrow$ $F(L(C))$, d'où les propriétés (1) et (2).

On suppose désormais que la catégorie $\mathcal{C}$ est cocomplète et que la monade $O L$ vérifie $\left(\mathcal{Q}_{1}\right)$. Nous allons voir que l'oubli $\mathcal{C}(O L) \rightarrow \mathcal{C}$ est alors en quelque sorte la meilleure approximation de $O$ par un foncteur commutant aux coégalisateurs de 1-complexes.

Soit $\left(\mathcal{D}^{\prime}, O^{\prime}: \mathcal{D}^{\prime} \rightarrow \mathcal{C}\right)$ une catégorie cocomplète au dessus de $\mathcal{C}$ telle que $O^{\prime}$ commute aux coégalisateurs de 1-complexes, et soit $F: L(\mathcal{C}) \rightarrow \mathcal{D}^{\prime}$ un foncteur tel 
que la composée $O^{\prime} F$ est naturellement isomorphe à la restriction de $O$ à $L(\mathcal{C})$. La proposition A.2.2 montre que $F$ s'étend en un foncteur $\widetilde{F}: \mathcal{C}(O L) \rightarrow \mathcal{D}^{\prime}$.

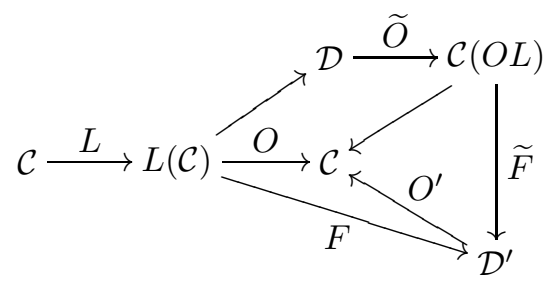

LEMME A.2.3

(a) On a pour toute $O L$-algèbre $M$ un isomorphisme naturel $O^{\prime} \widetilde{F}(M) \cong O M$.

(b) Supposons $F$ défini sur $\mathcal{D}$ entier; alors on a pour tout $M \in \mathcal{D}$ un morphisme naturel $\widetilde{F} \widetilde{O}(M) \rightarrow F(M)$ qui est un isomorphisme si $M$ est dans $\mathcal{L}_{\mathcal{D}}$.

Démonstration. - L'image par $O^{\prime} \widetilde{F}$ d'une $O L$-algèbre $M$ est le coégalisateur dans $\mathcal{C}$ du 1-complexe $O^{\prime} F(L O L(M) \leftrightarrows L(M))$ par construction de $\widetilde{F}$ et puisque $O^{\prime}$ commute aux coégalisateurs de 1-complexes. Comme la composée $O^{\prime} F$ est isomorphe à $O$ et comme $O M$ est le coégalisateur (canoniquement scindé) dans $\mathcal{C}$ du 1-complexe $(O L)^{2}(M) \leftrightarrows O L(M)$, on en déduit un isomorphisme naturel $O^{\prime} \widetilde{F}(M) \simeq O M$.

Pour le point (b) soit $M$ un objet de $\mathcal{D}$. L'image par $F$ de la counité $\epsilon(M)$ : $L O(M) \rightarrow M$ se factorise par l'épimorphisme $F(L O(M)) \rightarrow \widetilde{F} \widetilde{O}(M)$, d'où un morphisme $\widetilde{F} \widetilde{O}(M) \rightarrow F(M)$ naturel en $M$. Le point (b) du lemme A.2.1 montre que ce morphisme est un isomorphisme si $M$ est dans $\mathcal{L}_{\mathcal{D}}$.

Proposition A.2.4. - Soient $\mathcal{C}$ et $\mathcal{D}$ deux catégories cocomplètes et $O: \mathcal{D} \rightarrow \mathcal{C}$ un foncteur admettant un adjoint à gauche $L$. On suppose que $O$ commute aux coégalisateurs de 1-complexes et qu'un morphisme de $\mathcal{D}$ est un isomorphisme dès que son image par $O$ est un isomorphisme; alors la monade $O L$ vérifie $\left(\mathcal{Q}_{1}\right)$ et le foncteur $\mathcal{D} \rightarrow \mathcal{C}(O L)$ est une équivalence de catégories d'inverse $\left(\operatorname{Id}_{\mathcal{D}} \tilde{)}\right.$.

Démonstration. - Le foncteur $L$ commute aux colimites puisqu'il admet un adjoint à droite. On en déduit que la composée $O L$ commute aux coégalisateurs de 1-complexes donc vérifie $\left(\mathcal{Q}_{1}\right)$.

On dispose des foncteurs $\widetilde{O}: \mathcal{D} \rightarrow \mathcal{C}(O L)$ et $\left(\operatorname{Id}_{\mathcal{D}} \widetilde{{ }^{2}}: \mathcal{C}(O L) \rightarrow \mathcal{D}\right.$. Pour tout objet $M$ de $\mathcal{D}$ le diagramme $(L O)^{2}(M) \leftrightarrows(L O)(M) \rightarrow M$ est coégalisateur car son image par $O$ est coégalisateur (canoniquement scindé) dans $\mathcal{C}$ et $O$ reflète les isomorphismes. On en déduit que le morphisme naturel $\left(\operatorname{Id}_{\mathcal{D}}\right) \widetilde{O}(M) \rightarrow M$ est iso. D'autre part pour toute $O L$-algèbre $M$ on dispose d'un diagramme coégalisateur $L O L(M) \rightleftarrows L(M) \rightarrow$ $\left(\operatorname{Id}_{\mathcal{D}}\right)^{\widetilde{(}}(M)$ par définition de $\left(\operatorname{Id}_{\mathcal{D}}\right)^{\sim}$. On en déduit un morphisme $M \rightarrow \widetilde{O}\left(\operatorname{Id}_{\mathcal{D}}\right)^{\widetilde{T}}(M)$ puisque $M$ est le coégalisateur dans $\mathcal{C}$ et $\mathcal{C}(O L)$ du 1-complexe $(O L)^{2}(M) \leftrightarrows(O L)(M)$. Comme $O$ commute aux coégalisateurs de 1-complexes et comme $\widetilde{O}$ est un relèvement de $O$, ce morphisme est un isomorphisme dans $\mathcal{C}$ donc un isomorphisme dans $\mathcal{C}(O L)$. 
Voici un cas particulier : Soient $T$ et $T^{\prime}$ deux monades sur $\mathcal{C}$ vérifiant $\left(\mathcal{Q}_{1}\right)$.

Lemme A.2.5. - Un foncteur $\mathcal{C}(T) \rightarrow \mathcal{C}\left(T^{\prime}\right)$ au dessus de $\mathcal{C}$ commute aux coégalisateurs de 1-complexes donc équivaut à la donnée d'un foncteur $T(\mathcal{C}) \rightarrow \mathcal{C}\left(T^{\prime}\right)$ au dessus de $\mathcal{C}$, ou encore à la donnée d'une transformation naturelle $T^{\prime} \rightarrow T$ compatible avec les structures de monade de $T$ et $T^{\prime}$.

Démonstration. - Soit $R: \mathcal{C}(T) \rightarrow \mathcal{C}\left(T^{\prime}\right)$ un foncteur au dessus de $\mathcal{C}$. Soient $M_{1} \stackrel{\rightleftarrows}{\leftrightarrows} M_{0}$ un 1-complexe de $T$-algèbres, $M$ son coégalisateur dans $\mathcal{C}(T)$ et $M^{\prime}$ le coégalisateur dans $\mathcal{C}\left(T^{\prime}\right)$ du 1-complexe image par $R$. On obtient un morphisme $M^{\prime} \rightarrow R(M)$, lequel est iso dans $\mathcal{C}$ puisque les foncteurs oubli commutent aux coégalisateurs de 1-complexes, donc iso dans $\mathcal{C}\left(T^{\prime}\right)$. Autrement dit $R$ commute aux coégalisateurs de 1-complexes. Puisqu'une $T$-algèbre $M$ est le coégalisateur dans $\mathcal{C}(T)$ du 1-complexe $T^{2}(M) \leftrightarrows T(M)$ de $T(\mathcal{C}), R$ est donné par sa restriction à $T(\mathcal{C})$.

A toute structure naturelle de $T^{\prime}$-algèbre sur $T(C), C \in \mathcal{C}$, correspond un morphisme $T^{\prime}(C) \rightarrow T(C)$, adjoint de l'unité $C \rightarrow T(C)$ et compatible avec les structures de monade de $T$ et $T^{\prime}$. Inversement si $T^{\prime} \rightarrow T$ est un morphisme entre monades, on définit une structure de $T^{\prime}$-algèbre sur les objets $T(C)$ comme celle donnée par la composée $T^{\prime}(T(C)) \rightarrow T^{2}(C) \rightarrow T(C)$.

Soit $R: \mathcal{C}(T) \rightarrow \mathcal{C}\left(T^{\prime}\right)$ un foncteur au dessus de $\mathcal{C}$. Pour toute paire d'objets $C \in \mathcal{C}$ et $N \in \mathcal{C}(T)$ on dispose des bijections naturelles

$$
\operatorname{Hom}_{\mathcal{C}\left(T^{\prime}\right)}\left(T^{\prime}(C), R(N)\right) \simeq \operatorname{Hom}_{\mathcal{C}}(C, N) \simeq \operatorname{Hom}_{\mathcal{C}(T)}(T(C), N)
$$

d'où on déduit un foncteur $L: T^{\prime}(\mathcal{C}) \rightarrow \mathcal{C}(T), T^{\prime}(C) \mapsto T(C)$, lequel s'étend en un foncteur $\widetilde{L}: \mathcal{C}\left(T^{\prime}\right) \rightarrow \mathcal{C}(T)$. Comme pour toute $T^{\prime}$-algèbre $M$ l'objet $\widetilde{L}(M)$ est le coégalisateur du 1-complexe $\widetilde{L}\left(T^{\prime 2}(M) \leftrightarrows T^{\prime}(M)\right)$, les bijections qui précèdent induisent une bijection naturelle $\operatorname{Hom}_{\mathcal{C}\left(T^{\prime}\right)}(M, R N) \simeq \operatorname{Hom}_{\mathcal{C}(T)}(\widetilde{L} M, N)$ de sorte que $\widetilde{L}$ est adjoint à gauche de $R$. La proposition A.2.4 montre alors que le foncteur $\widetilde{R}: \mathcal{C}(T) \rightarrow \mathcal{C}\left(T^{\prime}\right)(R \widetilde{L})$ est une équivalence de catégories.

On a montré :

Corollaire A.2.6. - Soient $\mathcal{C}$ une catégorie cocomplète, $T$ et $T^{\prime}$ deux monades sur $\mathcal{C}$ vérifiant $\left(\mathcal{Q}_{1}\right)$ et $R: \mathcal{C}(T) \rightarrow \mathcal{C}\left(T^{\prime}\right)$ un foncteur au dessus de $\mathcal{C}$. Alors $R$ commute aux coégalisateurs de 1-complexes, admet un adjoint à gauche $L$ et le foncteur associé $\widetilde{R}: \mathcal{C}(T) \rightarrow \mathcal{C}\left(T^{\prime}\right)(R L)$ est une équivalence de catégorie.

Remarque. - Soient $\mathcal{C}$ et $\mathcal{D}$ deux catégories cocomplètes et $O: \mathcal{D} \rightarrow \mathcal{C}$ un foncteur admettant un adjoint à gauche $L$. On suppose que la monade $O L$ vérifie $\left(\mathcal{Q}_{0}\right)$. On peut alors montrer que la catégorie $\mathcal{C}(O L)$ est équivalente à la catégorie des 1-complexes de $\mathcal{L}_{\mathcal{D}}$ et classes d'homotopie en degré 0 de morphismes entre 1-complexes. 
A.3. Application : Monades et catégories abéliennes. - Soit $\mathcal{C}$ une catégorie possédant les limites et colimites finies. On suppose que $\mathcal{C}$ vérifie la condition (i) de la proposition A.1.2. La catégorie $\mathcal{C}_{\mathrm{ab}}$ des objets en groupe abélien de $\mathcal{C}$ est alors abélienne et les coégalisateurs dans $\mathcal{C}$ de 1 -complexes de $\mathcal{C}_{\text {ab }}$ sont scindés dans $\mathcal{C}$ (proposition A.1.2).

Soit $T$ une monade sur $\mathcal{C}$. Pour toute paire $C, C^{\prime}$ d'objets de $\mathcal{C}$ l'ensemble $\operatorname{Hom}_{\mathcal{C}(T)}\left(T(C), T\left(C^{\prime}\right)\right) \simeq \operatorname{Hom}_{\mathcal{C}}\left(C, T\left(C^{\prime}\right)\right)$ a une structure de groupe abélien naturelle en $C \in \mathcal{C}$ et $T\left(C^{\prime}\right) \in \mathcal{C}(T)$ si et seulement si la restriction de l'oubli $\mathcal{C}(T) \rightarrow \mathcal{C}$ à la sous-catégorie pleine $T(\mathcal{C})$ de $\mathcal{C}(T)$ se relève en un foncteur $A: T(\mathcal{C}) \rightarrow \mathcal{C}_{\mathrm{ab}}$, ce qui équivaut encore à ce que le foncteur $T$ et la transformation naturelle $T^{2} \rightarrow T$ soient à valeurs dans $\mathcal{C}_{\mathrm{ab}}$. (On n'en déduit pas que cette structure sur $\operatorname{Hom}_{\mathcal{C}(T)}\left(T(C), T\left(C^{\prime}\right)\right.$ ) est naturelle en $T(C) \in \mathcal{C}(T)$.)

Supposons cette condition satisfaite. La monade $T$ vérifie alors la condition $\left(\mathcal{Q}_{0}\right)$ par la proposition A.1.4 donc $\mathcal{C}(T)$ est cocomplète et l'oubli $\mathcal{C}(T) \rightarrow \mathcal{C}$ commute aux coégalisateurs de 1-complexes (proposition A.1.5).

Le foncteur $A: T(\mathcal{C}) \rightarrow \mathcal{C}_{\text {ab }}$ s'étend en un foncteur $\widetilde{A}: \mathcal{C}(T) \rightarrow \mathcal{C}_{\text {ab }}$ au dessus de $\mathcal{C}$ avec les propriétés :

- $\widetilde{A}$ commute aux coégalisateurs de 1-complexes (par la proposition A.1.6);

- $\widetilde{A}$ commute aux limites.

Par adjonction, l'image par $T$ de l'objet initial de $\mathcal{C}$ est l'objet initial de $\mathcal{C}(T)$. Notons 0 un objet final de $\mathcal{C}$; on vérifie que 0 a une unique structure de $T$-algèbre et que celle-ci en fait l'objet final de $\mathcal{C}(T)$.

Une deuxième condition pour que $\mathcal{C}(T)$ soit additive est que l'unique morphisme $T(\varnothing) \rightarrow 0$ soit un isomorphisme dans $\mathcal{C}(T)$, ce qui équivaut à ce que le morphisme $T(\varnothing) \rightarrow 0$ soit un isomorphisme dans $\mathcal{C}$, ou encore à ce que $\widetilde{A}(T(\varnothing)) \rightarrow 0$ soit un isomorphisme dans $\mathcal{C}_{\text {ab }}$.

Supposons cette condition satisfaite et soient $M$ et $N$ deux $T$-algèbres. On dispose d'un morphisme canonique de la somme dans $\mathcal{C}(T)$ de $M$ et $N$ dans leur produit, défini par les morphismes identité de $M$ et $N$ et les morphismes nuls $M \rightarrow 0 \simeq T(\varnothing) \rightarrow N$ et $N \rightarrow T(\varnothing) \rightarrow M$. Une troisième condition pour que la catégorie $\mathcal{C}(T)$ soit additive est que ce morphisme soit un isomorphisme, ce qui équivaut à demander que le morphisme image par $\widetilde{A}$ soit un isomorphisme dans $\mathcal{C}_{\text {ab }}$. La proposition A.1.6 montre que ce morphisme est un isomorphisme quels que soient $M$ et $N$ si (et seulement si) la composée $\widetilde{A} \circ T$ transforme la somme de deux objets de $\mathcal{C}$ en la somme des images dans $\mathcal{C}_{\mathrm{ab}}$, c'est à dire en le produit des images dans $\mathcal{C}$.

Supposons cette troisième condition satisfaite. Pour toute paire $M, N$ de $T$ algèbres, l'ensemble $\operatorname{Hom}_{\mathcal{C}(T)}(M, N)$ possède alors une structure naturelle de monoïde abélien qui coïncide avec sa structure de groupe abélien si $M$ et $N$ sont dans $T(\mathcal{C})$. Comme le diagramme $T^{2}(M) \leftrightarrows T(M) \rightarrow M$ est coégalisateur scindé dans $\mathcal{C}$, il en est 
de même du diagramme image par le foncteur

$$
\operatorname{Hom}_{\mathcal{C}(T)}(T(C),-) \simeq \operatorname{Hom}_{\mathcal{C}}(C,-)
$$

pour tout $C \in \mathcal{C}$ de sorte que le monoïde $\operatorname{Hom}_{\mathcal{C}(T)}(T(C), M)$ est un groupe. De même son image par le foncteur $\operatorname{Hom}_{\mathcal{C}(T)}(-, N)$, pour tout $N \in \mathcal{C}(T)$, est un diagramme égalisateur de sorte que $\operatorname{Hom}_{\mathcal{C}(T)}(M, N)$ est un groupe. Autrement dit la catégorie $\mathcal{C}(T)$ est additive.

Rappelons qu'un foncteur $R$ de $\mathcal{C}(T)$ dans une catégorie additive $\mathcal{B}$ est additif si et seulement si $R$ transforme la somme de deux $T$-algèbres en la somme des images (cf. [Mac, VIII, §2]). Puisque $T$ vérifie $\left(\mathcal{Q}_{0}\right)$, la proposition A.1.6 devient :

Proposition A.3.1. - Soit $\mathcal{C}$ une catégorie possédant toutes les limites et colimites finies et vérifiant la condition (i) de la proposition A.1.2. Soient $T$ une monade sur $\mathcal{C}$ telle que $\mathcal{C}(T)$ soit additive, et $R$ un foncteur de $\mathcal{C}(T)$ dans une catégorie additive $\mathcal{B}$. On suppose que pour toute $T$-algèbre $M$ l'image par $R$ du diagramme $T^{2}(M) \leftrightarrows T(M) \rightarrow M$ est coégalisateur. Alors $R$ est additif si et seulement si $R \circ \widetilde{T}: \mathcal{C} \rightarrow \mathcal{B}$ transforme la somme de deux objets de $\mathcal{C}$ en la somme des images, auquel cas $R$ est exact à droite.

Soit maintenant $M \rightarrow N$ un morphisme de $T$-algèbres. On dispose d'un morphisme canonique de $T$-algèbres

$$
\operatorname{Coker}(\operatorname{Ker}(M \rightarrow N) \rightarrow M) \longrightarrow \operatorname{Ker}(N \rightarrow \operatorname{Coker}(M \rightarrow N))
$$

dont l'image par $\widetilde{A}$ est un isomorphisme puisque $\widetilde{A}$ est exact, donc qui est iso dans $\mathcal{C}$ donc iso dans $\mathcal{C}(T)$. Autrement dit la catégorie $\mathcal{C}(T)$ est abélienne.

On a montré :

Proposition A.3.2. - Soit $\mathcal{C}$ une catégorie possédant toutes les limites et colimites finies et vérifiant la condition (i) de la proposition A.1.2. Soit $T$ une monade sur $\mathcal{C}$; les conditions suivantes sont équivalentes :

(i) Le foncteur $T$ et la transformation naturelle $T^{2} \rightarrow T$ sont à valeurs dans $\mathcal{C}_{\mathrm{ab}}$ et $T: \mathcal{C} \rightarrow \mathcal{C}$ transforme les sommes finies en produits.

(ii) La catégorie $\mathcal{C}(T)$ est additive.

Si elles sont vérifiées, la monade $T$ vérifie $\left(\mathcal{Q}_{0}\right)$ et $\mathcal{C}(T)$ est abélienne.

Observons que sous les hypothèses de la proposition le foncteur $\operatorname{Hom}_{\mathcal{C}}(C,-) \operatorname{de} \mathcal{C}_{\mathrm{ab}}$ dans la catégorie des groupes abéliens est additif et exact (voir la démonstration de la proposition A.1.2). On en déduit qu'un morphisme de $T$-algèbres est un épimorphisme si et seulement si il est épi dans $\mathcal{C}$ et que les $T$-algèbres libres sont des objets projectifs dans $\mathcal{C}(T)$.

Soient $T$ et $T^{\prime}$ deux monades sur $\mathcal{C}$ telles que les catégories $\mathcal{C}(T)$ et $\mathcal{C}\left(T^{\prime}\right)$ soient abéliennes, et $R$ un foncteur $\mathcal{C}(T) \rightarrow \mathcal{C}\left(T^{\prime}\right)$ au dessus de $\mathcal{C}$. Le corollaire A.2.6 montre que $R$ commute aux limites donc $R$ est additif, et que $R$ commute aux coégalisateurs 
de 1-complexes donc $R$ est exact à droite ( $c f$. également la proposition A.1.6). Le foncteur $R$ admet un adjoint à gauche $L$ par ce même corollaire. $L$ est additif et exact à droite car il commute aux colimites. Le corollaire A.2.6 devient donc :

Proposition A.3.3. - Soit $\mathcal{C}$ une catégorie possédant toutes les limites et colimites finies et vérifiant la condition (i) de la proposition A.1.2. Soient $T$ et $T^{\prime}$ deux monades sur $\mathcal{C}$ telles que les catégories $\mathcal{C}(T)$ et $\mathcal{C}\left(T^{\prime}\right)$ soient abéliennes, et $R: \mathcal{C}(T) \rightarrow \mathcal{C}\left(T^{\prime}\right)$ un foncteur au dessus de $\mathcal{C}$. Alors $R$ est additif, exact, admet un adjoint à gauche $L$ et le foncteur associé $\widetilde{R}: \mathcal{C}(T) \rightarrow \mathcal{C}(T)(R L)$ est une équivalence de catégories.

\section{EXEMPLES}

- Soit $\Lambda$ un anneau, respectivement un anneau $\mathbb{Z}$-gradué. Notons $T(S)$ le $\Lambda$-module libre sur un ensemble, respectivement un ensemble gradué, $S$. Alors $T$ a une structure de monade et la catégorie des $T$-algèbres de $\mathcal{E} n$ s, respectivement $\mathcal{E}$ ns-gr, est la catégorie abélienne des $\Lambda$-modules.

- Soit $\Lambda$ un anneau (gradué). La catégorie des tours de $\Lambda$-modules, respectivement la catégorie des complexes de $\Lambda$-modules, coïncide avec la catégorie des $\Lambda[x]$-modules, où $x$ est un générateur de degré -1 , respectivement avec la catégorie des $\Lambda[x] / x^{2}$ modules.

- Soit $\mathcal{C}$ une catégorie possédant les limites et colimites finies et satisfaisant la condition (i) de la proposition A.1.2. Il suffit de construire pour tout objet $C \in \mathcal{C}$ un objet en groupe abélien libre sur $C$ pour obtenir que $\mathcal{C}_{\text {ab }}$ est une catégorie abélienne associée à une monade sur $\mathcal{C}$ (par la proposition A.2.4).

A.4. Résolutions. - Soit $\mathcal{C}$ une catégorie possédant les sommes et produits finis et en particulier un objet initial $\iota$. On note $\mathcal{C}_{0}$ la catégorie des objets de $\mathcal{C}$ au dessus de $\iota$ et, pour $C$ dans $\mathcal{C}, C_{+}$l'objet $C \times \iota$ de $\mathcal{C}_{0}$.

Un complexe de $\mathcal{C}_{0}$ est une suite d'objets $\left(C_{n}\right), n \in \mathbb{Z}$, et de morphismes $C_{n+1} \rightarrow$ $C_{n}$ dans $\mathcal{C}_{0}$ telle que $C_{n}$ est l'objet $\iota$ pour tout $n<0$ et telle que la composée $C_{n+1} \rightarrow C_{n} \rightarrow C_{n-1}$ est le morphisme trivial $C_{n+1} \rightarrow \iota \rightarrow C_{n-1}$ pour tout $n$. Il est dit augmenté s'il est muni d'un morphisme $C_{0} \rightarrow C$, pour un objet $C$ de $\mathcal{C}_{0}$, tel que la composée $C_{1} \rightarrow C_{0} \rightarrow C$ est triviale; on le note alors $C_{*} \rightarrow C$. La longueur d'un complexe augmenté $C_{*} \rightarrow C$ est la borne inférieure (éventuellement infinie) des entiers $n$ tels que $C_{k}$ est l'objet $\iota$ pour tout $k>n$.

On définit de même les notions duales de cocomplexe (relativement à un objet terminal de $\mathcal{C}$ ), de cocomplexe augmenté et de longueur d'un cocomplexe augmenté.

Soit $\mathcal{L}$ une classe d'objets en cogroupe abélien de $\mathcal{C}$ ou de façon équivalente, de $\mathcal{C}_{0}$ (i.e. chaque objet $L$ de $\mathcal{L}$ est muni d'un relèvement du foncteur $\operatorname{Hom}_{\mathcal{C}}(L,-)$ en un foncteur à valeurs dans la catégorie des groupes abéliens). Un complexe augmenté $C_{*} \rightarrow C$ est dit acyclique relativement à $\mathcal{L}$ si pour chaque objet $L$ de $\mathcal{L}$ le morphisme entre complexes de groupes abéliens (concentré en degré 0 pour le second) $\operatorname{Hom}_{\mathcal{C}_{0}}\left(L, C_{*}\right) \rightarrow \operatorname{Hom}_{\mathcal{C}_{0}}(L, C)$ induit un isomorphisme en homologie. On dit que 
deux morphismes $f, g$ entre complexes sont homotopes relativement à $\mathcal{L}$ si pour tout objet $L$ de $\mathcal{L}$ les morphismes $\operatorname{Hom}_{\mathcal{C}_{0}}(L, f)$ et $\operatorname{Hom}_{\mathcal{C}_{0}}(L, g)$ entre complexes de groupes abéliens sont homotopes.

Proposition A.4.1. - Soient $\mathcal{C}$ une catégorie possédant les sommes et produits finis, $\mathcal{L}$ une classe d'objets en cogroupe abélien de $\mathcal{C}$ et soient $L_{*} \rightarrow C$ et $D_{*} \rightarrow D$ deux complexes augmentés de $\mathcal{C}_{0}$. On suppose que $L^{n}$ est dans $\mathcal{L}$ pour tout $n$ et que $D_{*} \rightarrow D$ est acyclique relativement à $\mathcal{L}$; alors tout morphisme $C \rightarrow D$ se relève en un morphisme $L_{*} \rightarrow D_{*}$, unique à homotopie relativement à $\mathcal{L}$ près.

Un objet simplicial augmenté de $\mathcal{C}$ est un objet simplicial $C$. munit d'un morphisme de $C$. dans un objet simplicial constant $C$. On le note $C \bullet \rightarrow C$.

Soit $C$. un objet simplicial de $\mathcal{C}$ et supposons que les limites finies existent dans $\mathcal{C}$. On définit le complexe normalisé $\mathrm{N}_{*} C$ de $C$. par :

$-\mathrm{N}_{0} C=C_{0+}$,

- $\mathrm{N}_{n+1} C$ est la limite du diagramme formé des objets $C_{n+1}, C_{n}$ et $\iota$, des morphismes $\mathrm{d}_{i}: C_{n+1} \rightarrow C_{n}$ pour $i \geqslant 1$ et du morphisme initial $\iota \rightarrow C_{n}$,

- $\mathrm{N}_{n+1} C \rightarrow \mathrm{N}_{n} C$ est le morphisme induit par $\mathrm{d}_{0}$.

Observons que si $L$ est un objet en cogroupe abélien de $\mathcal{C}$ et $C$. un objet simplicial de $\mathcal{C}$ alors l'image par $\operatorname{Hom}_{\mathcal{C}_{0}}(L,-)$ du normalisé de $C$. est le complexe normalisé du groupe abélien simplicial $\operatorname{Hom}_{\mathcal{C}}\left(L, C_{\bullet}\right)$.

Rappelons que si $\mathcal{C}$ est abélienne alors le foncteur $C . \mapsto \mathrm{N}_{*} C$ préserve la relation d'homotopie sur les morphismes et qu'on dispose d'une équivalence d'homotopie canonique entre le complexe $C_{*}$ formé de la somme alternée des faces $\mathrm{d}_{i}$ et le complexe $\mathrm{N}_{*} C$ par la théorie de Dold-Kan. ( $C f .[\mathrm{Ma1}, \S 22]$.)

Un objet simplicial augmenté $C . \rightarrow C$ est dit acyclique relativement à une classe $\mathcal{L}$ d'objets en cogroupe abélien de $\mathcal{C}$ si $L$ de $\mathcal{C}$ le complexe augmenté $\mathrm{N}_{*} C \rightarrow C_{+}$est acyclique relativement à $\mathcal{L}$. Deux morphismes entre objets simpliciaux augmentés sont dit homotopes relativement à $\mathcal{L}$ si pour tout objet $L$ de $\mathcal{L}$ leurs images par le foncteur $\operatorname{Hom}_{\mathcal{C}_{0}}\left(L, \mathrm{~N}_{*}(-)\right)$ sont homotopes. Si $C . \rightarrow C$ est une équivalence d'homotopie entre objets simpliciaux de $\mathcal{C}$ (voir par exemple [Ma1, I, §5] pour cette notion), la théorie de Dold-Kan montre que $C . \rightarrow C$ est acyclique relativement à tout objet en cogroupe abélien de $\mathcal{C}$.

La proposition suivante est la version simpliciale de la proposition A.4.1 :

Proposition A.4.2. - Soient $\mathcal{C}$ une catégorie possédant les sommes et limites finies, $\mathcal{L}$ une classe d'objets en cogroupe abélien de $\mathcal{C}, L_{\bullet} \rightarrow C$ et $D . \rightarrow D$ deux objets simpliciaux augmentés de $\mathcal{C}$. On suppose que $L_{n}$ est dans $\mathcal{L}$ pour tout $n$ et que $D$. $\rightarrow D$ est acyclique relativement à $\mathcal{L}$; alors tout morphisme $C \rightarrow D$ se relève en un morphisme $L_{\bullet} \rightarrow D_{\bullet}$ unique à homotopie relativement à $\mathcal{L}$ près.

Démonstration. — Par induction sur les squelettes de $L_{\bullet}$. 
Monades et résolutions. - Soit $\mathcal{C}$ une catégorie possédant les limites et colimites finies et vérifiant la condition (i) de la proposition A.1.2. La catégorie $\mathcal{C}_{\text {ab }}$ est alors abélienne et pour tout $C \in \mathcal{C}$ le foncteur $\operatorname{Hom}_{\mathcal{C}}(C,-)$ de $\mathcal{C}_{\text {ab }}$ dans la catégorie des groupes abéliens est exact.

Soit $T$ une monade sur $\mathcal{C}$ telle que le foncteur $T$ et la transformation naturelle $T \circ T \rightarrow T$ sont à valeurs dans $\mathcal{C}_{\text {ab }}$. Alors pour tout objet $C \in \mathcal{C}$ la $T$-algèbre libre $T(C)$ est un objet en cogroupe abélien de $\mathcal{C}(T)$ naturellement en $C \in \mathcal{C}$ et un objet en groupe abélien de $\mathcal{C}$ naturellement en $T(C) \in \mathcal{C}(T)$.

Notons $\iota$ l'objet initial de $\mathcal{C}$; son image par $T$ est l'objet initial de $\mathcal{C}(T)$. Pour $M$ une $T$-algèbre au dessus de $T(\iota)$ on note $\widetilde{M}$ le noyau dans $\mathcal{C}_{\text {ab }}$ du morphisme $M \rightarrow T(\iota)$. La donnée d'un morphisme $T(C) \rightarrow M$ au dessus de $T(\iota)$ équivaut alors à la donnée d'un morphisme $C \rightarrow \widetilde{M}$ dans $\mathcal{C}$.

On prend pour $\mathcal{L}$ la classe des $T$-algèbres libres. Ce qui précède montre qu'un complexe augmenté $M_{*} \rightarrow M$ de $\mathcal{C}(T)_{0}$, respectivement un objet simplicial augmenté $M_{\bullet} \rightarrow M$ de $\mathcal{C}(T)$, est acyclique relativement à $\mathcal{L}$ si et seulement si le complexe de $\mathcal{C}_{\text {ab }}$ associé $\widetilde{M}_{*} \rightarrow \widetilde{M}$, respectivement $M_{*} \rightarrow M$, est acyclique. De même deux morphismes entre complexes de $\mathcal{C}(T)_{0}$, respectivement entre objets simpliciaux de $\mathcal{C}(T)$, sont homotopes relativement à $\mathcal{L}$ si et seulement si les morphismes de complexes de $\mathcal{C}_{\text {ab }}$ associés sont homotopes.

La structure de monade de $T$ permet d'associer à toute $T$-algèbre $M$ une $T$-algèbre libre simpliciale $T_{\bullet}(M)$ et un morphisme de $T_{\bullet}(M)$ dans la $T$-algèbre simpliciale constante $M$ qui est canoniquement une équivalence d'homotopie entre objets simpliciaux de $\mathcal{C}$ (voir par exemple $[\mathbf{M a 2}, \S 9]$ ). En particulier le complexe augmenté de $\mathcal{C}_{\text {ab }}$ associé $T_{*}(M) \rightarrow M$ est acyclique. On appelle $T_{\bullet}(M) \rightarrow M$ la $T$-résolution simpliciale canonique de $M$.

\section{B. Produits tensoriels et torsion}

Pour $n$ un entier et $S$ un ensemble gradué on note $\Sigma^{n} S$ l'ensemble gradué égal à $S^{k-n}$ en degré $k$. Le foncteur $\Sigma=\Sigma^{1}$ est un isomorphisme de la catégorie $\mathcal{E}$ ns-gr dans elle même, d'inverse $\Sigma^{-1}$.

On considère une monade $T$ sur $\mathcal{E}$ ns-gr vérifiant les deux conditions suivantes :

- La catégorie $\mathcal{E}$ ns-gr $(T)$ est abélienne.

- On a pour tout ensemble gradué $S$ un isomorphisme naturel $T(\Sigma S) \cong \Sigma T(S)$ dans $\mathcal{E}$ ns-gr commutant avec les transformations naturelles Id $\rightarrow T$ et $T \circ T \rightarrow T$.

Pour toute $T$-algèbre $M$ la composée $T(\Sigma M) \simeq \Sigma T(M) \rightarrow \Sigma M$ munit $\Sigma M$ d'une structure de $T$-algèbre naturelle en $M \in \mathcal{E}$ ns-gr $(T)$. De même la composée $T\left(\Sigma^{-1} M\right) \simeq \Sigma^{-1} T(M) \rightarrow \Sigma^{-1} M$ munit $\Sigma^{-1} M$ d'une structure naturelle de $T$ algèbre. Les endofoncteurs $\Sigma$ et $\Sigma^{-1}$ de $\mathcal{E}$ ns-gr se relèvent donc en des endofoncteurs de $\mathcal{E}$ ns-gr $(T)$ inverses l'un de l'autre. 
Soient $S$ et $S^{\prime}$ deux ensembles gradués. On définit l'ensemble gradué $S \otimes S^{\prime}$ par

$$
\left(S \otimes S^{\prime}\right)^{n}=\sqcup_{k+l=n} S^{k} \times S^{l} .
$$

Il s'identifie au coproduit $\sqcup_{s \in S} \Sigma^{|s|} S^{\prime}$, où $|s|$ désigne le degré d'un élément $s \in S$. La $T$-algèbre $T\left(S \otimes S^{\prime}\right)$ s'identifie au coproduit $\oplus_{s \in S} \Sigma^{|s|} T\left(S^{\prime}\right)$ ce qui en fait un foncteur en la $T$-algèbre $T\left(S^{\prime}\right)$. Symétriquement $T\left(S \otimes S^{\prime}\right)$ est un foncteur en $T(S)$.

Pour $n$ entier notons $S_{n}$ le sous-ensemble gradué de $S$ formé des éléments de degré inférieur ou égal à $n$. Alors la $T$-algèbre $T\left(S_{n}\right)$ est un facteur direct de $T(S)$ naturel en $T(S)$ (parce que $\mathcal{E} n$ s- $\operatorname{gr}(T)$ est additive), les $T\left(S_{n}\right), n \in \mathbb{Z}$, forment une tour d'épimorphismes et on a un isomorphisme $T(S) \simeq \lim _{n} T\left(S_{n}\right)$. On définit le produit tensoriel $T(S) \otimes_{T} T\left(S^{\prime}\right)$ comme la limite sur $n$ des $T$-algèbres $T\left(S_{n} \otimes S_{n}^{\prime}\right)$.

Observons que le couple $(T, T)$ hérite de $T$ d'une structure de monade sur $\mathcal{E}$ ns-gr $\times$ $\mathcal{E}$ ns-gr et que la catégorie abélienne produit $\mathcal{E} n s-\operatorname{gr}(T) \times \mathcal{E}$ ns-gr $(T)$ s'identifie à la catégorie des $(T, T)$-algèbres de $\mathcal{E} n s-g r \times \mathcal{E}$ ns-gr. Comme la monade $T$ vérifie $\left(\mathcal{Q}_{0}\right)$, il en est de même pour $(T, T)$.

La proposition A.2.2 montre que le produit tensoriel des éléments de $T(\mathcal{E}$ ns-gr $) \times$ $T(\mathcal{E}$ ns-gr) s'étend en un foncteur $\mathcal{E}$ ns-gr $(T) \times \mathcal{E}$ ns-gr $(T) \rightarrow \mathcal{E}$ ns-gr $(T)$, qu'on note encore $M, N \mapsto M \otimes_{T} N$, commutant au coégalisateurs de 1-complexes. Par construction le produit tensoriel $M \otimes_{T} N$ de deux $T$-algèbres $M$ et $N$ est le coégalisateur du 1complexe $T^{2}(M) \otimes_{T} T^{2}(N) \rightrightarrows T(M) \otimes_{T} T(N)$ induit par le début des résolutions simpliciales canoniques de $M$ et $N$. L'isomorphisme canonique $S \otimes S^{\prime} \cong S^{\prime} \otimes S$ pour $S, S^{\prime} \in \mathcal{E}$ ns-gr induit un isomorphisme $M \otimes_{T} N \cong N \otimes_{T} M$ dans $\mathcal{E}$ ns-gr $(T)$.

\section{Proposition B.1}

(a) Le foncteur $\mathcal{E}$ ns-gr $(T) \times \mathcal{E}$ ns-gr $(T) \rightarrow \mathcal{E}$ ns-gr $(T)$,

$$
(M, N) \longmapsto M \otimes_{T} N
$$

commute aux coégalisateurs de 1-complexes.

(b) Pour toute T-algèbre $M$ le foncteur $N \mapsto M \otimes_{T} N$ est additif et exact à droite.

Démonstration. - Le point (a) vient de ce que la monade $(T, T)$ sur la catégorie produit $\mathcal{E}$ ns-gr $\times \mathcal{E}$ ns-gr vérifie $\left(\mathcal{Q}_{0}\right)$ et du point (a) de la proposition A.1.6.

Pour le point (b) : le foncteur $N \mapsto M \otimes_{T} N$ est la composée du foncteur $N \mapsto$ $(M, N)$ de $\mathcal{E} n s-g r(T)$ dans la catégorie abélienne produit $\mathcal{E} n s-\operatorname{gr}(T) \times \mathcal{E} n s-\operatorname{gr}(T)$ avec le foncteur (non additif) $-\otimes_{T}-:$ Ens-gr $(T) \times \mathcal{E}$ ns-gr $(T) \rightarrow \mathcal{E}$ ns-gr $(T)$. Comme $N \mapsto$ $(M, N)$ est additif et exact, la composée $N \mapsto M \otimes_{T} N$ commute aux coégalisateurs de 1-complexes par le point (a). Il suffit donc par la proposition A.3.1 de montrer que le foncteur $S \mapsto M \otimes_{T} T(S)$ transforme la somme de deux ensembles gradués en la somme des images. C'est vrai lorsque $M$ est l'image par $T$ d'un ensemble gradué. Le cas général vient de ce que $M \otimes_{T} T(S)$ est par ce qui précède le coégalisateur du diagramme $T^{2}(M) \otimes_{T} T(S) \leftrightarrows T(M) \otimes_{T} T(S)$ et de la commutation des colimites entre elles. 
Soient $M$ et $N$ deux $T$-algèbres. Le complexe augmenté $T_{*}(M) \rightarrow M$ associé à la résolution simpliciale canonique de $M$ est une résolution libre de $M$ fonctorielle en $M$. On définit la $T$-algèbre graduée $\operatorname{Tor}_{*}^{T}(M, N)$ comme l'homologie du complexe $T_{*}(M) \otimes_{T} N$. L'invariance par homotopie d'une résolution libre de $M$ montre que pour toute résolution libre $M_{*} \rightarrow M$ de $M$, l'homologie du complexe $M_{*} \otimes_{T} N$ est canoniquement isomorphe à $\operatorname{Tor}_{*}^{T}(M, N)$. Par exactitude à droite du produit tensoriel, la $T$-algèbre $\operatorname{Tor}_{0}^{T}(M, N)$ s'identifie à $M \otimes_{T} N$.

Soient $0 \rightarrow M^{\prime} \rightarrow M \rightarrow M^{\prime \prime} \rightarrow 0$ une suite exacte de $T$-algèbres et $M_{*}^{\prime} \rightarrow M^{\prime}$, $M_{*}^{\prime \prime} \rightarrow M^{\prime \prime}$ des résolutions libres de $M^{\prime}$ et $M^{\prime \prime}$ respectivement.

LEMme B.2. - Il existe une suite de morphismes $M_{n+1}^{\prime} \oplus M_{n+1}^{\prime \prime} \rightarrow M_{n}^{\prime} \oplus M_{n}^{\prime \prime}$ pour $n \geqslant 0$ et $M_{0}^{\prime} \oplus M_{0}^{\prime \prime} \rightarrow M$ telle que les injections $M_{n}^{\prime} \rightarrow M_{n}^{\prime} \oplus M_{n}^{\prime \prime}$ et les projections $M_{n}^{\prime} \oplus M_{n}^{\prime \prime} \rightarrow M_{n}^{\prime \prime}$ induisent une suite exacte courte de complexes augmentés $0 \rightarrow$ $\left(M_{*}^{\prime} \rightarrow M^{\prime}\right) \rightarrow\left(M_{*}^{\prime} \oplus M_{*}^{\prime \prime} \rightarrow M\right) \rightarrow\left(M_{*}^{\prime \prime} \rightarrow M^{\prime \prime}\right) \rightarrow 0$, en particulier telle que le complexe augmenté $M_{*}^{\prime} \oplus M_{*}^{\prime \prime} \rightarrow M$ est une résolution libre de $M$.

Démonstration. - Comme $M_{0}^{\prime \prime}$ est projectif et $M \rightarrow M^{\prime \prime}$ est surjectif, le morphisme $M_{0}^{\prime \prime} \rightarrow M^{\prime \prime}$ se relève en un morphisme $M_{0}^{\prime \prime} \rightarrow M$ et on vérifie que le morphisme obtenu $M_{0}^{\prime} \oplus M_{0}^{\prime \prime} \rightarrow M$ est surjectif. Soit $n$ un entier positif et supposons construite une suite exacte $M_{n}^{\prime} \oplus M_{n}^{\prime \prime} \rightarrow \cdots \rightarrow M_{0}^{\prime} \oplus M_{0}^{\prime \prime} \rightarrow M \rightarrow 0$ compatible avec les suites exactes $M_{n}^{\prime} \rightarrow \cdots \rightarrow M^{\prime} \rightarrow 0$ et $M_{n}^{\prime \prime} \rightarrow \cdots \rightarrow M^{\prime \prime} \rightarrow 0$. Notons $K$ et $K^{\prime \prime}$ les noyaux des morphismes $M_{n}^{\prime} \oplus M_{n}^{\prime \prime} \rightarrow M_{n-1}^{\prime} \oplus M_{n-1}^{\prime \prime}$ et $M_{n}^{\prime \prime} \rightarrow M_{n-1}^{\prime \prime}$ respectivement si $n$ est strictement positif, des morphismes $M_{0}^{\prime} \oplus M_{0}^{\prime \prime} \rightarrow M$ et $M_{0}^{\prime \prime} \rightarrow M^{\prime \prime}$ sinon. Le morphisme $K \rightarrow K^{\prime \prime}$ induit par la projection $M_{n}^{\prime} \oplus M_{n}^{\prime \prime} \rightarrow M_{n}^{\prime \prime}$ est surjectif d'où on déduit l'existence d'un relèvement de $M_{n+1}^{\prime \prime} \rightarrow K^{\prime \prime}$ à $K$. On vérifie que le morphisme obtenu $M_{n+1}^{\prime} \oplus M_{n+1}^{\prime \prime} \rightarrow K$ est surjectif. On construit ainsi par récurrence sur $n$ une suite de morphismes $M_{n+1}^{\prime} \oplus M_{n+1}^{\prime \prime} \rightarrow M_{n}^{\prime} \oplus M_{n}^{\prime \prime}$ compatibles avec les morphismes $M_{n+1}^{\prime} \rightarrow M_{n}$ et $M_{n+1}^{\prime \prime} \rightarrow M_{n}^{\prime \prime}$. Le fait qu'elle forme une résolution de $M$ vient de la suite exacte longue en homologie associée à une suite exacte courte de complexes.

Soit maintenant $N$ une $T$-algèbre. Pour chaque entier $n$, la suite $0 \rightarrow M_{n}^{\prime} \otimes_{T} N \rightarrow$ $\left(M_{n}^{\prime} \oplus M_{n}^{\prime \prime}\right) \otimes_{T} N \rightarrow M_{n}^{\prime \prime} \otimes_{T} N \rightarrow 0$ est une suite exacte car scindée dans $\mathcal{E}$ ns-gr $(T)$. La suite exacte de complexes $0 \rightarrow M_{*}^{\prime} \otimes_{T} N \rightarrow\left(M_{*}^{\prime} \oplus M_{*}^{\prime \prime}\right) \otimes_{T} N \rightarrow M_{*}^{\prime \prime} \otimes_{T} N \rightarrow 0$ induit une suite exacte longue en homologie qui s'écrit

$\cdots \longrightarrow \operatorname{Tor}_{1}^{T}(M, N) \longrightarrow \operatorname{Tor}_{1}^{T}\left(M^{\prime \prime}, N\right) \longrightarrow M^{\prime} \otimes_{T} N \longrightarrow M \otimes_{T} N \longrightarrow M^{\prime \prime} \otimes_{T} N \longrightarrow 0$.

En particulier le foncteur $M \mapsto M \otimes_{T} N$ est exact à gauche si la $T$-algèbre $\operatorname{Tor}_{1}^{T}(M, N)$ est nulle quelque soit $M$. Le lemme suivant, qui s'obtient également par la suite exacte longue des $\operatorname{Tor}^{T}(-, N)$, donne la réciproque :

Lemme B.3. - Soient $M$ une T-algèbre, $M_{0} \rightarrow M$ le début d'une résolution libre de $M$ et $K$ le noyau du morphisme $M_{0} \rightarrow M$; alors pour tout entier $n$ strictement positif 
l'objet $\operatorname{Tor}_{n+1}^{T}(M, N)$ s'identifie à $\operatorname{Tor}_{n}^{T}(K, N)$ et $\operatorname{Tor}_{1}^{T}(M, N)$ s'identifie au noyau du morphisme $K \otimes_{T} N \rightarrow M_{0} \otimes_{T} N$.

Soient symétriquement $M$ une $T$-algèbre, $M_{*} \rightarrow M$ une résolution libre de $M$ et $0 \rightarrow N^{\prime} \rightarrow N \rightarrow N^{\prime \prime} \rightarrow 0$ une suite exacte de $T$-algèbres. Cette dernière induit une suite exacte de complexes $M_{*} \otimes_{T} N^{\prime} \rightarrow M_{*} \otimes_{T} N \rightarrow M_{*} \otimes_{T} N^{\prime \prime} \rightarrow 0$.

Proposition B.4. - Soit $n$ un entier positif tel que les objets $\operatorname{Tor}_{1}^{T}\left(N^{\prime \prime}, M_{k}\right)$ sont nuls pour tout $0 \leqslant k \leqslant n$; alors la suite exacte de complexes $M_{*} \otimes_{T} N^{\prime} \rightarrow M_{*} \otimes_{T} N \rightarrow$ $M_{*} \otimes_{T} N^{\prime \prime} \rightarrow 0$ induit une suite exacte

$$
\begin{aligned}
\operatorname{Tor}_{n+1}^{T}(M, N) \longrightarrow \operatorname{Tor}_{n+1}^{T}\left(M, N^{\prime \prime}\right) \longrightarrow \operatorname{Tor}_{n}^{T}\left(M, N^{\prime}\right) \longrightarrow \cdots & \longrightarrow M \otimes_{T} N \longrightarrow M \otimes_{T} N^{\prime \prime} \longrightarrow 0 .
\end{aligned}
$$

Démonstration. - Notons $K_{n}$ l'image du morphisme $M_{n+1} \rightarrow M_{n}$ et $K$ le noyau du morphisme $K_{n} \otimes_{T} N \rightarrow K_{n} \otimes_{T} N^{\prime \prime}$. Notons $A_{*}^{\prime}, A_{*}$ et $A_{*}^{\prime \prime}$ les complexes tronqués $\cdots \rightarrow 0 \rightarrow K \rightarrow M_{n} \otimes_{T} N^{\prime} \rightarrow \cdots \rightarrow M_{0} \otimes_{T} N^{\prime}, \cdots 0 \rightarrow K_{n} \otimes_{T} N \rightarrow M_{n} \otimes_{T} N \rightarrow \cdots \rightarrow$ $M_{0} \otimes_{T} N$ et $\cdots 0 \rightarrow K_{n} \otimes_{T} N^{\prime \prime} \rightarrow M_{n} \otimes_{T} N^{\prime \prime} \rightarrow \cdots \rightarrow M_{0} \otimes_{T} N^{\prime \prime}$ respectivement. L'hypothèse portant sur les objets $\operatorname{Tor}_{1}^{T}\left(N^{\prime \prime}, M_{k}\right)$ garantit que la suite de complexes $0 \rightarrow A_{*}^{\prime} \rightarrow A_{*} \rightarrow A_{*}^{\prime \prime} \rightarrow 0$ est exacte. On en déduit une suite exacte longue en homologie. Les $k$-ièmes objets d'homologie des complexes $A_{*}$ et $A_{*}^{\prime \prime}$ s'identifient aux objets $\operatorname{Tor}_{k}^{T}(M, N)$ et $\operatorname{Tor}_{k}^{T}\left(M, N^{\prime \prime}\right)$ respectivement pour $0 \leqslant k \leqslant n+1$ (on utilise le lemme ci-dessus). De même le $k$-ième objet d'homologie du complexe $A_{*}^{\prime}$ s'identifie à l'objet $\operatorname{Tor}_{k}^{T}\left(M, N^{\prime}\right)$ pour $0 \leqslant k \leqslant n$ car le morphisme $K_{n} \otimes_{T} N^{\prime} \rightarrow M_{n} \otimes_{T} N^{\prime}$ se factorise par la surjection $K_{n} \otimes_{T} N^{\prime} \rightarrow K$. On obtient la suite exacte annoncée.

Corollaire B.5. - Soient $M$ et $N$ deux T-algèbres, $M_{*} \rightarrow M$ et $N_{*} \rightarrow N$ des résolutions libres de $M$ et $N$ respectivement et $n$ un entier positif. On suppose que les objets $\operatorname{Tor}_{k+1}^{T}\left(M, N_{l}\right)$ et $\operatorname{Tor}_{k+1}^{T}\left(N, M_{l}\right)$ sont nuls pour $0 \leqslant k, l \leqslant n$; alors les objets $\operatorname{Tor}_{k}^{T}(M, N)$ et $\operatorname{Tor}_{k}^{T}(N, M)$ sont naturellement isomorphes pour $0 \leqslant k \leqslant n$.

Démonstration. - Pour $n=0$ notons $K$ le noyau du morphisme $N_{0} \rightarrow N$. On dispose par la proposition d'une suite exacte

$$
\operatorname{Tor}_{1}^{T}\left(M, N_{0}\right) \longrightarrow \operatorname{Tor}_{1}^{T}(M, N) \longrightarrow M \widehat{\otimes} K \longrightarrow M \widehat{\otimes} N_{0} \longrightarrow M \widehat{\otimes} N \longrightarrow 0
$$

d'où on déduit que l'objet $\operatorname{Tor}_{1}^{T}(M, N)$ s'identifie au noyau du morphisme $M \widehat{\otimes} K \rightarrow$ $M \widehat{\otimes} N_{0}$ donc à l'objet $\operatorname{Tor}_{1}^{T}(N, M)$ par le lemme B.3.

Pour $n \geqslant 1$ notons $K$ le conoyau du morphisme $N_{n+1} \rightarrow N_{n}$. On obtient par la proposition et par récurrence sur $n$ un isomorphisme $\operatorname{Tor}_{n+1}^{T}(M, N) \cong \operatorname{Tor}_{1}^{T}(M, K)$. Ce qui précède donne un isomorphisme $\operatorname{Tor}_{1}^{T}(M, K) \cong \operatorname{Tor}_{1}^{T}(K, M)$. Or $\operatorname{Tor}_{1}^{T}(K, M)$ est naturellement isomorphe à $\operatorname{Tor}_{n+1}^{T}(N, M)$ par le lemme B.3 


\section{Limites et dérivés}

Soit $\mathcal{A}$ une catégorie abélienne où les produits dénombrables sont exacts et soit $\left(M_{s}, g_{s}: M_{s} \rightarrow M_{s-1}\right)$ une tour d'objets de $\mathcal{A}$. On forme le morphisme

$$
\mathrm{Id}-\left(g_{s}\right): \prod_{s} M_{s} \longrightarrow \prod_{s} M_{s}
$$

qu'on voit comme un complexe de $\mathcal{A}$ concentré en degré 0 et 1 . Son homologie en degré 1 s'interprète comme la limite $M_{\infty}$ des $M_{s}$. Celle en degré 0 s'interprète comme l'objet $\lim _{s}^{1} M_{s}$. Toute suite exacte courte $0 \rightarrow\left(M_{s}^{\prime}\right) \rightarrow\left(M_{s}\right) \rightarrow\left(M_{s}^{\prime \prime}\right) \rightarrow 0$ de tours donne naissance à une suite exacte courte de complexes donc à une suite exacte

$$
0 \longrightarrow M_{\infty}^{\prime} \longrightarrow M_{\infty} \longrightarrow M_{\infty}^{\prime \prime} \longrightarrow \lim _{s}^{1} M_{s}^{\prime} \longrightarrow \lim _{s}^{1} M_{s} \longrightarrow \lim _{s}^{1} M_{s}^{\prime \prime} \longrightarrow 0
$$

dans $\mathcal{A}$ : le foncteur $\lim ^{1}$ est exact à droite.

Plus généralement soit $A_{*, 1} \rightarrow A_{*, 0}$ un morphisme entre complexes d'une catégorie abélienne $\mathcal{A}$, qu'on regarde comme un bicomplexe $\mathbb{N} \times \mathbb{N}$-gradué.

On note $\mathrm{H}_{*}^{\mathrm{h}}\left(A_{*, t}\right)$ ou $\mathrm{H}_{*}^{\mathrm{h}} A$ l'homologie du bicomplexe par rapport au premier indice et $\mathrm{H}_{*}^{v} A$ celle par rapport au second. Au bicomplexe $A_{*, *}$ on associe le complexe total $\operatorname{Tot}_{*} A=A_{*-1,1} \oplus A_{*, 0}$ muni de la différentielle habituelle. Ce dernier possède deux filtrations croissantes et convergentes $\mathrm{F}_{s} \operatorname{Tot}_{*} A=\oplus_{s^{\prime}+t=*, s^{\prime} \leqslant s} A_{s^{\prime}, t}$ et $\mathrm{F}_{t}^{\prime} \operatorname{Tot}_{*} A=$ $\oplus_{s+t^{\prime}=*, t^{\prime} \leqslant t} A_{s, t^{\prime}}$ donnant naissance à deux suites spectrales convergeant fortement vers l'homologie de $\operatorname{Tot}_{*} A$ (voir par exemple $[\mathbf{C E}]$ ).

La première est de terme $\mathrm{E}_{s, *}^{1}=\mathrm{H}_{*}\left(\mathrm{~F}_{s} / \mathrm{F}_{s-1}\right)$,

$$
\mathrm{E}_{s, t}^{2}=\mathrm{H}_{s}^{\mathrm{h}} \mathrm{H}_{t-s}^{\mathrm{v}} A .
$$

Sa différentielle $\mathrm{d}^{r}$ est de bidegré $(-r,-1)$. On dispose d'un morphisme de bord $\mathrm{E}_{0, t}^{2} \rightarrow$ $\mathrm{H}_{t}$ Tot $A$ et la suite $\left(\mathrm{E}_{s, t}^{r}\right)_{r}$ converge fortement vers le gradué d'une filtration finie de $\mathrm{H}_{t} \operatorname{Tot} A$.

Comme $A_{s, t}$ est nul si $t$ est différent de 0 et 1 , le terme $\mathrm{E}_{s, t}^{2}$ est concentré sur les lignes $t=s$ et $t=s+1$ et la seule différentielle éventuellement non nulle est la différentielle $\mathrm{d}^{2}: \mathrm{E}_{s+2, s+2}^{2} \rightarrow \mathrm{E}_{s, s+1}^{2}, s \geqslant 0$. En particulier la suite spectrale dégénère au terme $\mathrm{E}^{3}$; on a un isomorphisme $\mathrm{E}_{0,0}^{2} \rightarrow \mathrm{H}_{0}$ Tot $A$ et plus généralement une suite exacte longue

$$
\mathrm{E}_{s+2, s+2}^{2} \longrightarrow \mathrm{E}_{s, s+1}^{2} \longrightarrow \mathrm{H}_{s+1} \operatorname{Tot} A \longrightarrow \mathrm{E}_{s+1, s+1}^{2} \longrightarrow \mathrm{E}_{s-1, s}^{2} .
$$

La seconde suite spectrale est de terme $\mathrm{E}_{*, t}^{\prime 1}=\mathrm{H}_{*}\left(\mathrm{~F}_{s}^{\prime} / \mathrm{F}_{s-1}^{\prime}\right)$,

$$
\mathrm{E}_{s, t}^{\prime 2}=\mathrm{H}_{t}^{\mathrm{v}} \mathrm{H}_{s-t}^{\mathrm{h}} A
$$

concentré sur les lignes $t=0$ et $t=1$. Sa différentielle $\mathrm{d}^{\prime r}$ est de bidegré $(-1,-r)$ donc la suite spectrale dégénère au terme $\mathrm{E}^{\prime 2}$. On obtient pour tout entier $s$ une suite exacte

$$
0 \longrightarrow \mathrm{E}_{s, 0}^{\prime 2} \longrightarrow \mathrm{H}_{s} \operatorname{Tot} A \longrightarrow \mathrm{E}_{s, 1}^{\prime 2} \longrightarrow 0 .
$$

Ces deux suites spectrales nous permettent d'obtenir la 
Proposition C.6. - Soit $A_{*, 1} \rightarrow A_{*, 0}$ un morphisme entre complexes d'une catégorie abélienne $\mathcal{A}$, qu'on regarde comme un bicomplexe de $\mathcal{A}$.

(a) On a un isomorphisme $\mathrm{H}_{0}^{\mathrm{v}} \mathrm{H}_{0}^{\mathrm{h}} A \simeq \mathrm{H}_{0}^{\mathrm{h}} \mathrm{H}_{0}^{\mathrm{v}} A$.

(b) Supposons que pour tout $s \geqslant 0$ l'objet $\mathrm{H}_{s}^{\mathrm{h}} \mathrm{H}_{0}^{\mathrm{v}} A$ est nul; alors on a une suite exacte

$$
0 \longrightarrow \mathrm{H}_{0}^{\mathrm{v}} \mathrm{H}_{s}^{\mathrm{h}} A \longrightarrow \mathrm{H}_{s-1}^{\mathrm{h}} \mathrm{H}_{1}^{\mathrm{v}} A \longrightarrow \mathrm{H}_{1}^{\mathrm{v}} \mathrm{H}_{s-1}^{\mathrm{h}} A \longrightarrow 0 .
$$

(c) Supposons que pour tout $t \in\{0,1\}$ et tout $s \geqslant 1$ l'objet $\mathrm{H}_{t}^{\mathrm{v}} \mathrm{H}_{s}^{\mathrm{h}} A$ est nul; alors on a une suite exacte

$$
0 \longrightarrow \mathrm{H}_{2}^{\mathrm{h}} \mathrm{H}_{1}^{\mathrm{v}} A \longrightarrow \mathrm{H}_{0}^{\mathrm{h}} \mathrm{H}_{1}^{\mathrm{v}} A \longrightarrow \mathrm{H}_{1}^{\mathrm{v}} \mathrm{H}_{0}^{\mathrm{h}} A \longrightarrow \mathrm{H}_{1}^{\mathrm{h}} \mathrm{H}_{0}^{\mathrm{v}} A \longrightarrow 0
$$

et un isomorphisme $\mathrm{H}_{s+2}^{\mathrm{h}} \mathrm{H}_{1}^{\mathrm{v}} A \simeq \mathrm{H}_{s}^{\mathrm{h}} \mathrm{H}_{1}^{\mathrm{v}} A$ pour $s \geqslant 1$.

Démonstration. - Le point (a) vient de l'isomorphisme $\mathrm{E}_{0,0}^{2} \simeq \mathrm{H}_{0}$ Tot $A$, du fait que le terme $\mathrm{E}_{0,1}^{\prime 2}$ est nul et de la suite exacte associée à la seconde suite spectrale.

Sous l'hypothèse du point (b) le terme $\mathrm{E}_{s, t}^{2}$ de la première suite spectrale est concentré sur la ligne $t=s+1$ donc cette suite spectrale dégénère au terme $\mathrm{E}^{2}$ et on a un isomorphisme $\mathrm{H}_{s}^{\mathrm{h}} \mathrm{H}_{1}^{\mathrm{v}} A \simeq \mathrm{H}_{s+1}$ Tot $A$. La suite exacte issue de la seconde suite spectrale donne alors la suite exacte cherchée.

Sous l'hypothèse du point (c) le terme $\mathrm{E}_{s, t}^{\prime 2}$ est nul si $s$ est différent de $t$. La suite exacte issue de la seconde suite spectrale donne les isomorphismes $\mathrm{H}_{1}$ Tot $A \simeq \mathrm{H}_{1}^{\mathrm{v}} \mathrm{H}_{0}^{\mathrm{h}} A$ et $\mathrm{H}_{s} \operatorname{Tot} A \simeq 0$ si $s>1$. La suite exacte longue issue de la première suite spectrale permet de conclure.

Application. - Soient $\mathcal{A}$ une catégorie abélienne où les produits dénombrables sont exacts et $\left(M_{k, l}\right)_{k, l}$ une tour de $\mathcal{A}$ indexée par $\mathbb{N} \times \mathbb{N}$. A chaque entier $k$ correspond une tour $\left(M_{k, l}\right)_{l}$ donc un complexe $\prod_{l} M_{k, l} \rightarrow \prod_{l} M_{k, l}$ concentré en degré 0 et 1 . A la tour de complexes $\left(\prod_{l} M_{k, l} \rightarrow \prod_{l} M_{k, l}\right)_{k}$ on associe le bicomplexe

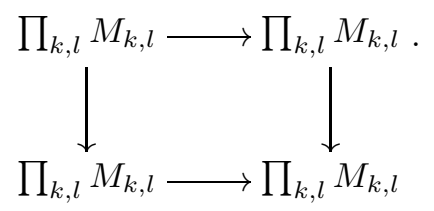

concentré en bidegrés dans $\{0,1\} \times\{0,1\}$ et qu'on prend pour $A_{*, *}$. Pour $s, t$ dans $\{0,1\}$ le terme $\mathrm{H}_{1-s}^{\mathrm{h}} \mathrm{H}_{1-t}^{\mathrm{v}} A_{*, *}$ s'interprète comme l'objet $\lim _{k}^{s} \lim _{l}^{t} M_{k, l}$ et le terme $\mathrm{H}_{1-t}^{\mathrm{v}} \mathrm{H}_{1-s}^{\mathrm{h}} A_{*, *}$ comme l'objet $\lim _{l}^{t} \lim _{k}^{s} M_{k, l}$. On obtient avec les points (a) et (b) de la proposition qui précède la

Proposition C.7. - Soient $\mathcal{A}$ une catégorie abélienne où les produits dénombrables sont exacts et $\left(M_{k, l}\right)_{k, l}$ une tour de $\mathcal{A}$ indexée par $\mathbb{N} \times \mathbb{N}$; alors

(a) On a un isomorphisme $\lim _{k}^{1} \lim _{l}^{1} M_{k, l} \cong \lim _{l}^{1} \lim _{k}^{1} M_{k, l}$. 
(b) Supposons que le terme $\lim _{l}^{1} M_{k, l}$ est nul pour tout $k$; alors on a une suite exacte

$$
0 \longrightarrow \lim _{l}^{1} M_{\infty, l} \longrightarrow \lim _{k}^{1} M_{k, \infty} \longrightarrow \lim _{l} \lim _{k}^{1} M_{k, l} \longrightarrow 0 .
$$

Typiquement pour le point $(\mathrm{b})\left(M_{k, l}\right)_{l}$ est la tour associée à une filtration décroissante d'un objet $M_{k}$ donc est une tour de surjections donc est sans $\lim ^{1}$. 


\section{BIBLIOGRAPHIE}

[Ad1] J.F. AdAms - Lectures on Generalized Cohomology Theories, Lect. Notes in Math., vol. 99, Springer, 1969.

[Ad2] Stable Homotopy and Generalized Homology, University of Chicago Press, 1974.

[AM] M. Artin \& B. Mazur - Etale Homotopy, Lect. Notes in Math., vol. 100, Springer, 1969.

[BB] M. BARR \& J. BECK - «Homology and standard constructions », in Seminar on triples and Categorical Homology Theory, Lect. Notes in Math., vol. 80, Springer, 1969, p. 245-335.

[BCM] M. Bendersky, E.B. Curtis \& H.R. Miller - « The Unstable Adams Spectral Sequence for Generalized Homology », Topology 3 (1978), p. 229 248.

[Boa1] J.M. BoARdman - «Stable Operations in Generalized Cohomology », in Handbook of algebraic topology, 1995, p. 585-686.

[Boa] _ « Conditionally Convergent Spectral Sequences », Contemp. Math., vol. 239, 1999, p. 49-84.

[BJW] J.M. BoARdman, D.C. Johnson \& W.S. Wilson - « Unstable Operations in Generalized Cohomology », in Handbook of algebraic topology, 1995, p. 687828.

[Bor] F. Borceux - Handbook of categorical algebra, 2. Categories and structures, Cambridge University Press, 1994.

[B] Bourbaki - Algèbre, chap. X, Masson, 1980.

[Bou1] A.K. Bousfield - «Nice homology coalgebra», Trans. Amer. Math. Soc. 148 (1970), p. 473-489. 
[Bou2] — " On the homology spectral sequence of a cosimplicial space », Amer. J. Math. 109 (1987), p. 361-394.

[BK] A.K. Bousfield \& D.M. KAN - Homotopy Limits, Completions, and Localizations, Lect. Notes in Math., vol. 304, Springer, 1972.

[CE] H. Cartan \& S. Eilenberg - Homological Algebra, Princeton University Press, 1956.

[CS] P.E. Conner \& L. SMith - « On the complex bordism of finite complexes », Publ. Math. Inst. Hautes Études Sci. 37 (1969), p. 117-221.

[DL] F.-X. Dehon \& J. Lannes - « Sur les espaces fonctionnels dont la source est le classifiant d'un groupe de Lie compact commutatif», Publ. Math. Inst. Hautes Études Sci. 89 (1999), p. 127-177.

[Dr] A. Dress - «Zur Spectralsequenz von Faserungen», Invent. Math. 3 (1967), p. $172-178$.

[DS] E. Dror Farjoun \& J. Smith - « A Geometric Interpretation of Lannes' Functor T », in Théorie de l'homotopie, Astérisque, vol. 191, 1990, p. 87-95.

[DwS] W.G. Dwyer \& J. Splalinski - « Homotopy Theories and Model Categories », in Handbook of algebraic topology, 1995, p. 73-126.

[GJ] P.G. Goerss \& J.F. Jardine - Simplicial Homotopy Theory, Progress in Math., vol. 174, Birkäuser, 1999.

[KW] N. Kunn \& M. Winstead - « On the Torsion in the Cohomology of Certain Mapping Spaces », Topology 35 (1996), p. 875-881.

[Lan] P.S. Landweber - «Coherence, Flatness and Cobordism of Classifying Spaces », in Proc. adv. Study Inst. alg. Topol., 1970, p. 256-269.

[La1] J. LANnes - «Sur les espaces fonctionnels dont la source est le classifiant d'un p-groupe abélien élémentaire», Publ. Math. Inst. Hautes Études Sci. $\mathbf{7 5}$ (1992), p. 135-244.

[La2] _ «Divers aspects des opérations de Steenrod», in Journée en l'honneur de Henri Cartan, Journée Annuelle, Société Mathématique de France, 1997, p. $18-27$.

[Mac] S. MaC LANE - Categories for the Working Mathematician, Springer, 1971.

[Ma1] P. MAY - Simplicial Objects in Algebraic Topology, University of Chicago Press, 1967.

[Ma2] - The geometry of iterated loop spaces, Lect. Notes in Math., vol. 271, Springer, 1972. 
[Mo1] F. Morel - « Quelques remarques sur la cohomologie modulo $p$ des pro- $p$ espaces et les résultats de Jean Lannes concernant les espaces fonctionnels $\operatorname{hom}(\mathrm{BV}, X) »$, Ann. scient. Éc. Norm. Sup. 4e série 26 (1993), p. 309-360.

[Mo2] _ « «nsembles profinis simpliciaux et interprétation géométrique du foncteur T», Bull. Soc. math. France 124 (1996), p. 347-373.

[Qu1] D.G. QuilLen - Homotopical Algebra, Lect. Notes in Math., vol. 43, Springer, 1967.

[Qu2] _ «An Application of Simplicial Profinite Groups », Comment. Math. Helv. 44 (1969), p. 45-60.

[RW] D.C. RaVenel \& W.S. Wilson - « The Hopf ring for complex cobordism », J. Pure Appl. Algebra 9 (1976), p. 241-280.

[RWY] D.C. Ravenel, W.S. Wilson \& N. Yagita - «Brown-Peterson cohomology from Morava $K$-theory », K-Theory 15 (1998), p. 147-199.

[Re] D. ReCtor - «Steenrod operations in the Eilenberg-Moore spectral sequence», Comment. Math. Helv. 45 (1970), p. 540-552.

[Sc] L. Schwartz - Unstable Modules over the Steenrod Algebra and Sullivan's Fixed Point Set Conjecture, University of Chicago Press, 1994.

[Seg] G. Segal - « Classifying Spaces and Spectral Sequences », Publ. Math. Inst. Hautes Études Sci. 34 (1968), p. 105-112.

[Su] D. Sullivan - « Genetics of Homotopy Theory and the Adams Conjecture», Ann. of Math. 100 (1974), p. 1-79.

[tD] T. TOM DIECK - « Bordism of $G$-manifolds and integrality theorems », Topology 9 (1970), p. 345-358.

[Wilk] C.W. WiLKERson - «lambda-ring, binomial domains, and vector bundles over $\mathbb{C P}^{\infty} »$, Comm. Algebra 10 (1982), p. 311-328.

[Wi] W.S. WILSON - «The $\Omega$-spectrum for Brown-Peterson Cohomology, Part I », Comment. Math. Helv. 48 (1973), p. 45-55. 



\section{INDEX}

$\mathrm{B} \pi, \mathrm{B} \mathbb{Z} / p^{n}, 20,91$

complexe, cocomplexe, 124

1-complexe, 1-cocomplexe, 112

$\triangle[n], 11$

$\mathcal{E}, 51$

Ens-gr, 37

filtration, 27, 30, 39, 42, 45

Fix, Fix $_{\mathcal{U}}, 91$

$\mathrm{F}^{n} M, 42$

G, 37, 46

$\mathrm{G}^{a}, \widetilde{\mathrm{G}}, 47$

$\mathrm{G}_{\mathrm{H}}, \widetilde{\mathrm{G}}_{\mathrm{H}}, 51$

G. $(M) \rightarrow M, 72$

$\operatorname{hom}_{\mathrm{pt}}(W, Y), 13$

$\operatorname{hom}(W, Y), 11$

$\operatorname{hom}(X, Y), 11$

$\mathrm{H} \pi^{*} X, 9$

$\mathrm{H}-\mathcal{U}, 91$

$\Lambda_{n}, 61$

$\mathrm{K}^{a}(L), 48$

$\mathcal{K}_{\mathrm{H}}, \mathcal{K}_{\mathrm{H}_{-},}, 51$

$\mathrm{K}_{\mathrm{H}}(E), 51$

$\mathrm{K}^{\bullet}(M), 72$

$\mathcal{K}_{\mathrm{MU}}, \mathcal{K}_{\mathrm{MU}-}, 46,47$

$\mathrm{K}(S), 37$

$\widehat{\mathcal{L}}, 28,38$

$\widehat{\mathrm{L}}, 38$

$\widehat{\mathrm{L}}_{n}, 62$

$\lim _{s}^{1}, 130$

$M / \mathrm{f}^{1}, 27,39,52$

$M / \mathrm{F}^{n}, 42,49$

$M / \mathrm{f}^{n}, 27,39$

$M \widehat{\otimes} N, 36,43,50$

$M \otimes_{\widehat{\mathrm{L}}_{n}} N, 63,127$

$M_{+}, 47$
$\widetilde{M}, 47$

$\mathcal{M}, 27$

$\mathcal{M}_{\mathrm{f}}, 27$

$\mathcal{M}_{\mathrm{fc}}, 27$

$\widehat{\mathcal{M}}, 38$

$\widehat{\mathcal{M}}^{0}, 39$

$\widehat{\mathcal{M}}_{n}, 62$

$\mathrm{M}_{n}^{n^{\prime}}(M), 63$

$(M: P)_{\mathcal{K}_{\mathrm{MU}}}, 84$

$\widehat{\mathrm{MU}}_{n}, 23$

$\widehat{\mathrm{MU}}^{*}, 23,27$

$\widehat{\mathrm{MU}}^{*} X, \widetilde{\mathrm{MU}}^{*} X, 23,30,44,46$

$\left(\widehat{\mathrm{MU}}^{*} X\right) / \mathrm{f}^{n}, 30,45$

$\Omega X, 12$

$p, 7$

$\pi_{0}\left(M_{\bullet}\right), 72$

$\pi_{0} X, 13$

$\pi_{k} X, 16$

pro- $\mathcal{C}, 9$

$\left(\mathcal{Q}_{0}\right), 115$

résolution, 57, 72, 73, 126

$\mathrm{R} X, 10$

sans $p$-torsion, 30

$\mathcal{S}, 7$

$\widehat{\mathcal{S}}, \mathrm{h} \widehat{\mathcal{S}}, 9$

$\widehat{\mathcal{S}}_{\mathrm{pt}}, \mathrm{h} \widehat{\mathcal{S}}_{\mathrm{pt}}, 11$

$\Sigma M, 44,126$

$\Sigma X, 12$

$\mathrm{Sk}_{n} X, 17$

$T$-algèbre, 114

$\mathrm{T}_{\mathrm{B} \pi}, \mathrm{T}_{n}, 86,94$

$\mathrm{T}\left(M / \mathrm{f}^{1}\right), \mathrm{T}_{\mathcal{U}}\left(M / \mathrm{f}^{1}\right), 90$

$T .(M) \rightarrow M, 126$

$T^{2}(M) \rightleftarrows T(M) \rightarrow M, 115$ 
$\operatorname{Tor}_{k}(M, N), 59$

$\operatorname{Tor}_{k}^{\widehat{\mathrm{L}}_{n}}(M, N), 64,128$

$\widehat{W}^{f}, 9$

$W \widehat{\wedge} X, W \widehat{\times} X, 13$

$\widehat{X}^{p}, 10$
$X \rightarrow \mathrm{K}^{\bullet}\left(\widehat{\mathrm{MU}}^{*} X\right), 72$

$|X|, 13$

$X_{+}, 11$

$X \wedge Z, 12$

$X_{\mathrm{h} \mathbb{Z} / p}, X^{\mathrm{h} \mathbb{Z} / p}, 20$

$\mathbb{Z} / p^{\infty}, 94$ 